\title{
Measuring executive and underlying cognitive functions in schoolchildren in Ukraine
}

Citation for published version (APA):

Toornstra, A. D. (2019). Measuring executive and underlying cognitive functions in schoolchildren in Ukraine: effects of demographic influences, regression based normative data and environmental variables in development. [Doctoral Thesis, Maastricht University]. Maastricht University. https://doi.org/10.26481/dis.20191204at

Document status and date:

Published: 01/01/2019

DOI:

10.26481/dis.20191204at

Document Version:

Publisher's PDF, also known as Version of record

\section{Please check the document version of this publication:}

- A submitted manuscript is the version of the article upon submission and before peer-review. There can be important differences between the submitted version and the official published version of record.

People interested in the research are advised to contact the author for the final version of the publication, or visit the DOI to the publisher's website.

- The final author version and the galley proof are versions of the publication after peer review.

- The final published version features the final layout of the paper including the volume, issue and page numbers.

Link to publication

\footnotetext{
General rights rights.

- You may freely distribute the URL identifying the publication in the public portal. please follow below link for the End User Agreement:

www.umlib.nl/taverne-license

Take down policy

If you believe that this document breaches copyright please contact us at:

repository@maastrichtuniversity.nl

providing details and we will investigate your claim.
}

Copyright and moral rights for the publications made accessible in the public portal are retained by the authors and/or other copyright owners and it is a condition of accessing publications that users recognise and abide by the legal requirements associated with these

- Users may download and print one copy of any publication from the public portal for the purpose of private study or research.

- You may not further distribute the material or use it for any profit-making activity or commercial gain

If the publication is distributed under the terms of Article $25 \mathrm{fa}$ of the Dutch Copyright Act, indicated by the "Taverne" license above, 


\section{MEASURING EXECUTIVE AND}

\section{UNDERLYING COGNITIVE FUNCTIONS IN SCHOOLCHILDREN IN UKRAINE}

Effects of demographic influences, regression based normative data and environmental variables in development

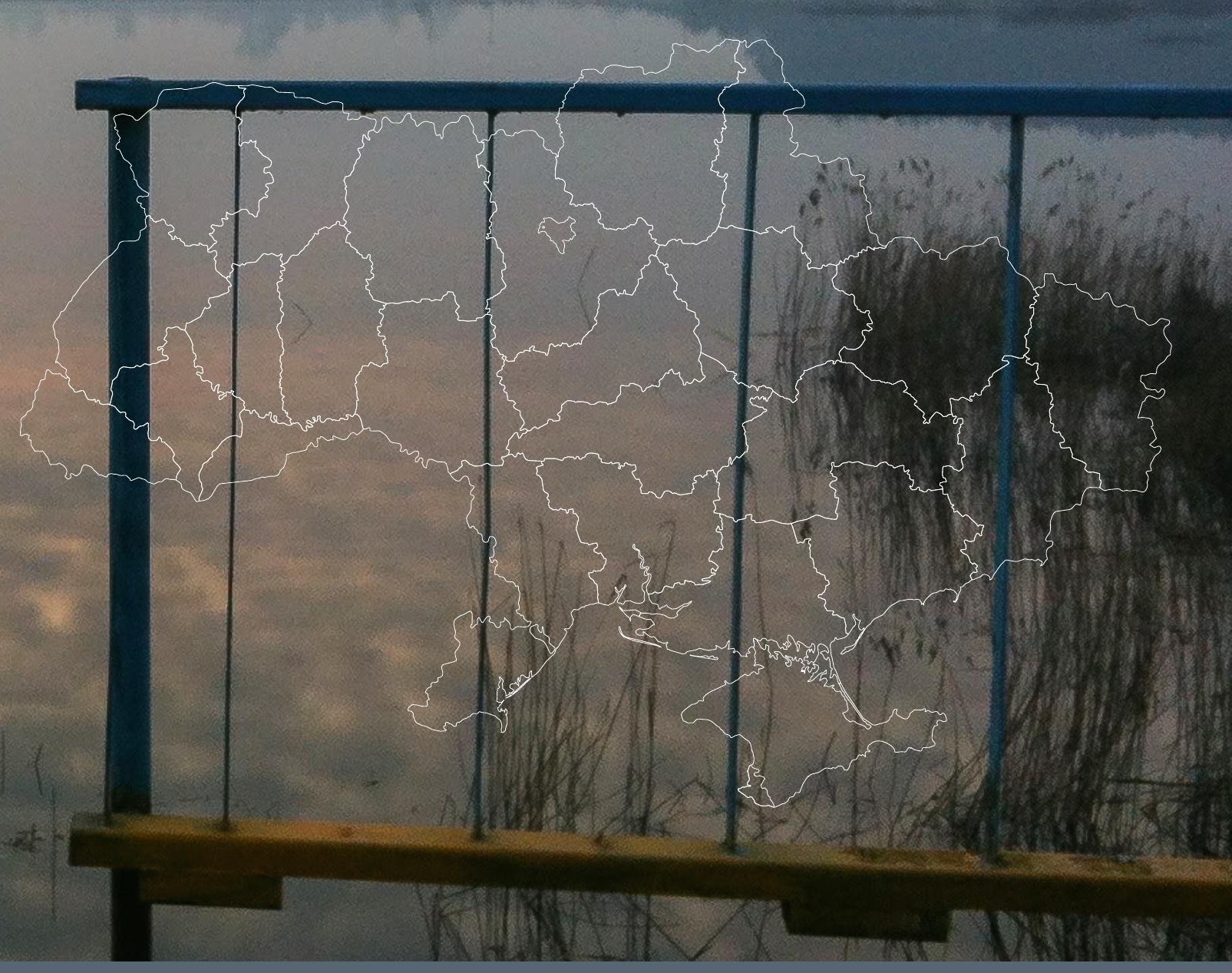

A. D. Toornstra 



\author{
Measuring executive and underlying cognitive \\ functions in schoolchildren in Ukraine; effects of \\ demographic influences, regression based normative data and \\ environmental variables in development
}


The research in this thesis was conducted at the Department of Work and Organisational Psychology at The Faculty of Psychology and Neuroscience, Maastricht University, The Netherlands and Governor Kremers Centre (GKC) - Maastricht University Medical Centre.

\section{GKC}

Maastricht University

Printing: Ridderprint BV, www.ridderprint.nl

Cover photo A. Toornstra, Ukraine

Cover design: Ridderprint BV, The Netherlands

ISBN: 978-94-6375-709-6

C Annette D. Toornstra, Maastricht 2019

All rights reserved. No parts of this book may be reproduced or transmitted in any form or by any means, without permission from the author or where appropriate, the publisher of the article. 
Measuring executive and underlying cognitive functions in schoolchildren in Ukraine; effects of demographic influences, regression based normative data and environmental variables in development

\section{DISSERTATION}

to obtain the degree of Doctor at Maastricht University, on the authority of the Rector Magnificus Prof. dr. Rianne M.

Letschert, in accordance with the decision of the Board of Deans, to be defended in public on December 4, 2019 , at 14.00 hours by Annette D. Toornstra 


\section{Supervisors}

Prof. dr. L. M. G. Curfs

Prof. dr. G. Kok

\section{Co-supervisors}

Dr. P. P. M. Hurks

Dr. K. Massar

Assessment committee

Prof. dr. R. A. C. Ruiter

Prof. dr. H. van den Borne

Dr. M. van Boxtel

Prof. Dr. J. Scheres

Prof. Dr. R. Reis 


\section{TABLE OF CONTENTS}

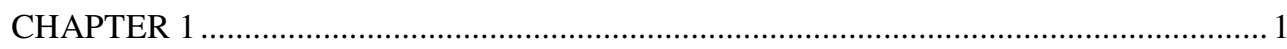

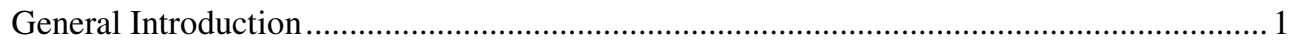

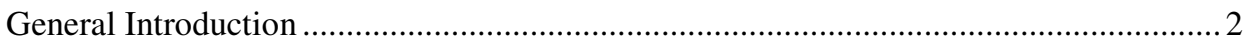

\section{CHAPTER 2}

Measuring visual matching and short-term recognition memory with the CANTAB ${ }^{\circledR}$ Delayed Matching to Sample task in schoolchildren: Effects of demographic influences, multiple outcome measures and regression based normative data................................... 13

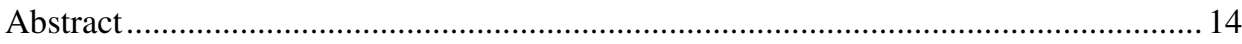

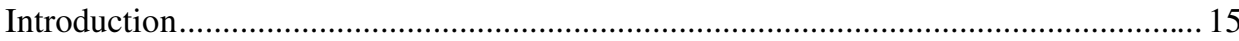

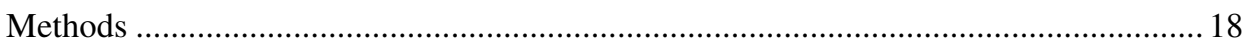

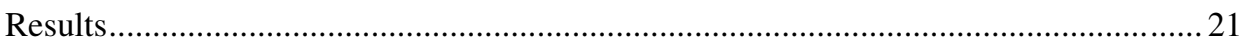

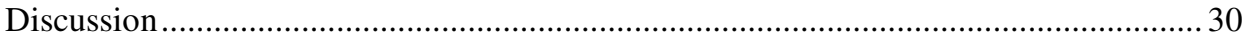

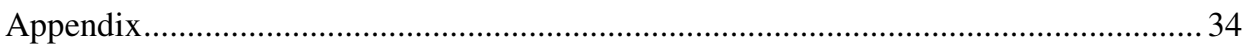

\section{CHAPTER 3}

Measuring visual, spatial, and visual spatial short-term memory in schoolchildren: studying the influence of age, sex, and level of parental education and demographically corrected norms

Abstract. 62

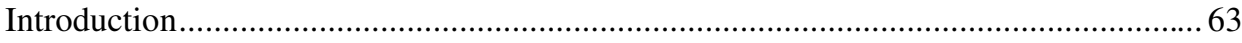

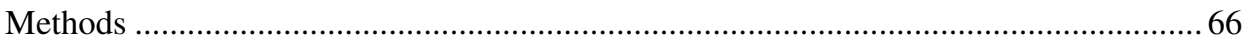

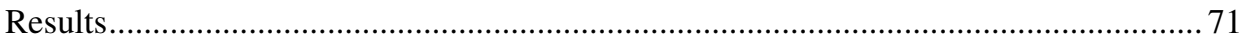

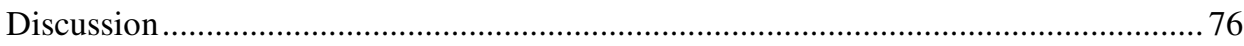

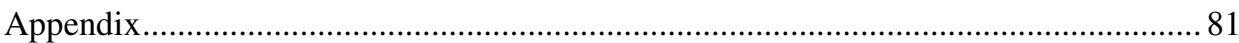

\section{CHAPTER 4}

Measuring goal setting in school-aged children: studying the influence of age, sex, and level of parental education and demographically corrected norms

Abstract

Introduction

Methods

Results.

Discussion 
Appendix.

\section{CHAPTER 5}

Perceptions of alcohol and alcohol use in Western Ukraine: A study among community members and young adults

Abstract

Introduction

Methods

Results

Discussion

Appendix

CHAPTER 6

"I am not my father": Alcohol dependence and associated stigma in Ukraine

Abstract

Introduction

Methods 157

Results. 160

Discussion

CHAPTER 7

General Discussion

General Discussion 176

Summary. 185

Samenvatting 188

Valorization 192

References

Acknowledgements. 




\section{CHAPTER 1}

General Introduction 


\section{General Introduction}

This thesis focuses on schoolchildren in Western Ukraine and consists of two parts. In the first part of this thesis the focus is on collecting data to establish demographically corrected norms for executive (and underlying cognitive) functions tests in a sample of schoolchildren in Southwest Ukraine. In the second part of this thesis, perceptions of alcohol use and possible alcohol dependence were explored using a qualitative approach. This chapter starts with an introduction on cognitive functions deemed central to learning in school aged children and measurement of these functions. Next, the qualitative investigation of perceptions on alcohol use is introduced. The last part of this chapter provides an overview of the thesis.

Learning during school age is important for a variety of life outcomes. Competencies from primary school years onward in reading and mathematics have for one, been linked to academic success later in life, employability, and level of income (Baer et al., 2009; Duncan et al., 2007; Parsons \& Bynner, 1997). Success in reading and mathematics learning in turn has been associated with increased cognitive abilities such as attention, inhibition and working memory (Geary, 2013), which are considered to be part of the cluster of skills called 'executive functions' (EFs; Diamond, 2013). Diamond (2013) defined EFs as an umbrella term for a set of cognitive skills that prevent one to respond at a moment when inhibition might be required, meet demands to cope with novel challenges, plan actions and consider concepts from several perspectives, which affect cognitive, social and emotional domains of life.

Measuring and monitoring EF development therefore is a way to gauge the learning process and progression in school age children. Indeed, studies have shown that stimulating executive functions leads to improvement in (early) learning processes (Diamond, 2012; Zelazo, Blair \& Willoughby, 2016), although the results are not consistent. Because of the relevance of executive functions for learning, professionals in the fields of psychology, education and neuroscience extensively research EFs. This requires valid and reliable EFs tools, or tests and a way of interpreting test results in a meaningful manner (Mitrushina et al., 2005; Zelazo et al., 2016). The thesis contributes to this need, by constructing suitable norm data for several EFs tests that offer professionals a way to relate an individual's test performance to that of others. The first part of this dissertation concerned the collection of normative data for measurement of executive functions in a non-Western sample of Ukrainian schoolchildren aged 5.5 - 14.5 years old. Normative data for EF varying measurements have been established, which mostly tend to be based on Western samples. Good normative test data should take into consideration demographic variables, such as the society and culture in which the individual lives which in turn affect thinking, knowledge, values and beliefs (Ardila, 2006; Van der Elst, Hurks, Wassenberg, Meijs \& Jolles, 2011).

Ukraine is a multi-ethnic society with its own demographic characteristics, that emphasize the need for suitable test norms for this group specifically (Rosselli \& Ardila, 2003). Ukraine's complex history included shifting borders (e.g., at the end of WW II) and 
(forced) migration (Aliyev, 2019; Haukkala, 2015; Maass, 2019; Onwijn, 2005, p. 40-58). Ukraine is a middle-income country, yet $3.3 \%$ of the population lives in significant poverty ( $<\$ 5$ per day) and the group just above this, that is moderate poverty, was $22 \%$ of the population in 2015 (World Bank, 2016). Moderate poverty was not expressed in monetary values, but linked to the (increased) proportion of income spent on basic needs such as heating. Children may experience poverty, may need to work in the vegetable garden that many households have or do other work (e.g., family business), and/or a parent may work abroad for longer periods. Parent's socio economic status has been associated with the level of parental education which in turn affects children's cognitive functions and academic achievement (Davis-Kean, 2005; Mezzacappa, 2004; Naglieri \& Ronning, 2000a; Noble, McCandliss, \& Farah, 2007; White, 1982). Furthermore, Ukraine has been described as having a culture that values hospitality and celebrations associated with a pattern of family meals accompanied by wine consumption including children (Popova, Rehm, Patra, \& Zatonski, 2007).

The context in which children learn is important. The development of children and EFs specifically, is linked to their environment. Bronfenbrenner (1994) visualized this in a model of ever widening concentric circles with the child in the center. Specifically, he proposed proximate influences such as family and school in the first layers surrounding the center. Ever widening circles are proposed to represent social and cultural influences affecting development, such as the community, media, social services, and local policies. The most outer layer represents attitudes and norms in a society. One of the premises of Bronfenbrenner's (1994) theory was to show how complex development is: There are numerous different behaviors, beliefs, attitudes, and norms influencing a child, who in turn has its own biological characteristics, creating a wide range of possible interactive effects on child development. Thus, children in West Ukraine and the characteristics of their environment may give rise to specific characteristics in their learning processes.

The focus in the first part of the thesis is on collecting data to establish demographically corrected norms for EFs tests in a sample of schoolchildren in West Ukraine. In the second part of the dissertation some of the 'outer layers' of Bronfenbrenner's (1994) model are investigated, delving deeper into these Ukrainian children's families, communities and society. First, EF measurement is discussed in sections 1.1 and 1.2. Then the qualitative investigation is introduced (section 1.3) and finally an outline of the dissertation is provided in section 1.4.

\subsection{Executive functions and academic performance}

EFs is an umbrella term for a group of cognitive skills employed for example when it is beneficial not to follow one's impulse in a situation, engage in mental activities in relation to novel situations, solving problems and the planning and execution of those plans (Diamond, 2013). EFs have been defined as a set of interrelated processes or functions involving attentional control, information processing, cognitive flexibility, and 


\section{Chapter 1}

goal setting (Anderson, 2002; Miyake et al., 2000). EFs are not a single unified group or brain area but are rather thought to be closely related yet distinct functions, depicted by Anderson as four boxes in a large square (2002, p.73). He visualized these four boxes, each as a sub-group of functions (Figure 1). Attentional Control, a box positioned in the bottom left corner, feeds into the other three boxes (one-way arrows). Attentional Control contains the sub-components of selective attention, self-regulation, self-monitoring, and inhibition. Attentional control is thus defined as the ability to direct one's attention to a specific task, retain this focus, inhibit other responses, and to monitor and regulate one's behavior so that steps to execute a task are completed in a correct manner and order (Anderson, 2002).

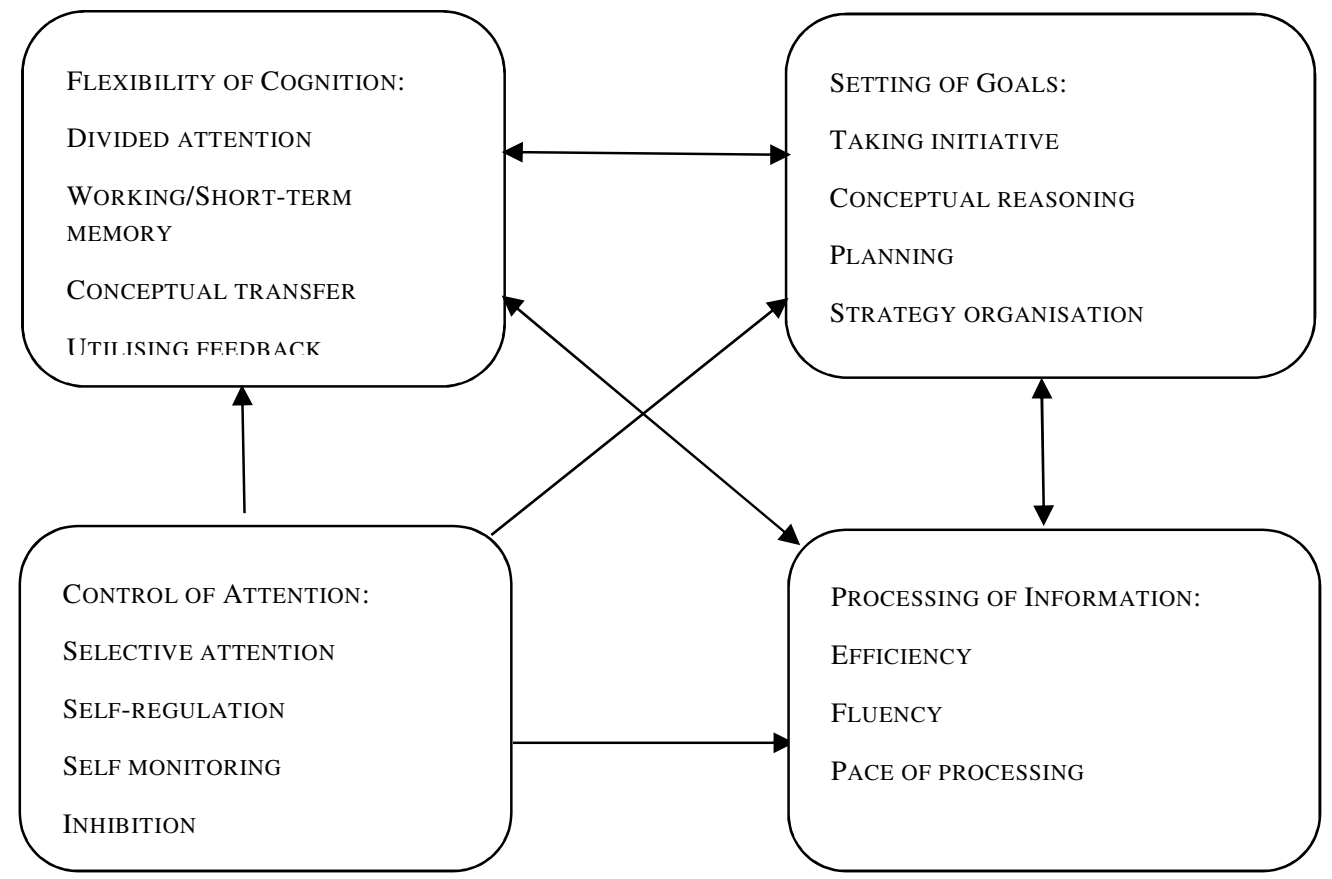

Figure 1. Proposed model of executive functions. Adapted from Anderson (2002), p. 73.

The other three boxes positioned in the rest of the quadrants, show mutually reciprocal connections (arrows). The first one is Information Processing i.e., the ability to interpret data (stimuli) in order to give a response that is suitable in the context and/or the level of difficulty of the stimuli that are being processed. The function of Information Processing thus consists of the sub-components of efficiency, fluency and speed (pace) of processing. The second box is Cognitive Flexibility, which is the ability to shift between task demands, and to adapt strategies based on identified mistakes. Efficient execution of these strategies also requires keeping information in short-term memory and attending to different sources of information. Other subcomponents are divided attention, working memory, conceptual transfer and feedback application. The third box, the function of Goal Setting, refers to the ability to generate new concepts, and to plan activities and strategies, which result in problem solving (Anderson, 2002). Goal Setting consists of the sub-functions of initiative, 
conceptual thinking, planning and strategic organization. To summarize this depiction: Attentional control is required for all other EFs and these in turn may reciprocally influence each other.

Each of these EFs is sub-served by specific yet closely connected brain networks, which are thought to develop not at the same time during childhood. Indeed, research has shown that these EFs mature from early childhood well into adolescence, although each show unique developmental trajectories (Anderson, 2002; Diamond, 2013). They are crucial in negotiating life outcomes, i.e., school, work, or health (Alloway \& Alloway, 2010; Bailey, 2007; Miller et al., 2011). For example, it is thought that e.g., attentional control develops earlier in childhood compared to the more complex skills of planning (Anderson, 2002).

Understanding development related to school readiness and academic success therefore, requires a careful evaluation of these different EFs (Diamond, 2013). To measure specific executive functions, the Cambridge Neuropsychological Test Automated Battery (CANTAB $^{\circledR}$; Cambridge Cognition, 2012) has been used in clinical and scientific research (Chien et al., 2015; Mattson et al., 2013). The CANTAB ${ }^{\circledR}$ is based on established traditional neuropsychological tests and worldwide psychometrics indicate validity (Robbins et al., 1998; Roque et al., 2011; Sahakian \& Owen, 1992). It was decided to administer this battery to test children's EFs in Ukrainian schools. However, in order to assess whether children's EF development is within the normal range, one needs to collect reference data on how children in Ukraine normally perform.

\subsection{Establishing norm scores among Ukrainian schoolchildren}

Test scores e.g., the number of items correctly answered by a child, provide little information if those cannot be related to the normal range of performance of a group. In line with the discussion above, attention is thought to develop from early infancy, but maturation with age implies that more and more complex functions develop over time, and that more stimuli in the environment can be attended to. This in turn also requires development of memory in order to consciously process complex information, plan ahead, and set goals (Sluzenski et al, 2004). The normal range of memory in for example the amount of information processed of a very young child differs from late adolescence (Gathercole et al., 2004). Knowing what score should be considered 'below the normal range' compared to children of the same age is important since this may inform whether there should be reasons for concern. There are to the best of our knowledge no norms available for the $\mathrm{CANTAB}^{\circledR}$ for children in Ukraine. $\mathrm{CANTAB}^{\circledR}$ norms for children are to the best of our knowledge mainly based on Western samples, except for few studies (Cambridge Cognition, 2012; De Luca et al., 2003; Green et al., 2019; Lehto, Juujärvi, Kooistra, \& Pulkkinen, 2003; Luciana \& Nelson, 2002; Roque, Teixeira, Zachi, \& Ventura, 2011). There is however reason to assume that cultural differences may exist in children's executive functions. 


\section{Chapter 1}

Culture has been defined as the feelings, beliefs, manner of thinking, values and attitudes shared in a group (Ardila, 2006). These in turn affect cognitive functioning, which implies that norms for one group (e.g., one culture) are not necessarily suitable for another group (Rosselli \& Ardila, 2003). Thus, in order to be able to determine the level of EF development in schoolchildren in Ukraine with some accuracy, CANTAB ${ }^{\circledR}$ norms for Ukraine need to be collected. That is the aim of the first three studies in this dissertation (chapter 2, 3, \& 4). In recent years, a relatively novel method to establish normative scores for a measurement tool has been introduced (Van Breukelen \& Vlaeyen, 2005). This regression-based method of norming will be briefly described below and it will be used in the research reported in this dissertation.

Traditionally, norms (including the norms of the measurement instrument utilized in the studies reported in this dissertation) are derived from age subgroups in a sample. That is, a sample is divided in e.g., two-year age subgroups (i.e., 6-7 years old, 8-9 years old etc.) and the means and standard deviations are calculated for each subgroup. This traditional method has some drawbacks, for example, the test scores for norming are in this method only applicable to a particular age group in the sample, and these exclude the results of all other children with different ages. Subgrouping means that a large sample size is needed to achieve statistical significance. Also, the boundaries of age groups may mean that children of almost the same age are in different age groups. For example, maintaining a two-year age subgroup e.g., means that a child of 5.9 years old would not fall in the 6-7 years old group, despite an age difference of only one month. This child's scores will fall in the 4-5 years old age subgroup, with an age difference of 1.9 years compared to the youngest children in this subgroup. Smaller age ranges in the subgroups alleviate this problem, but these require larger samples (Naglieri, 2003). Another disadvantage relates to sampling issues, i.e., an older group may sometimes perform worse compared to a younger age group due to certain (developmental) characteristics, which would then become the norm for a certain age group.

To avoid these disadvantages of traditional norming methods a novel and promising approach called continuous norming or regression-based norming was applied in chapters 2, 3, and 4 (in line with e.g., Van der Elst et al., 2011). Here, age is used as a continuous variable in the regression-based models, i.e., age is applied to each test score in the sample as opposed to traditional norming methods of creating certain age sub groups in the sample, e.g., 6-7 years old only (Bechger et al., 2009; Zachary \& Gorsuch, 1985). Applying a variable in this way to the whole sample leads to more stable norms (Van Breukelen \& Vlaeyen, 2005). This regression-based method of norming furthermore allows for the inclusion of categorical and continuous variables simultaneously in the analyses (Strauss et al., 2006, p. 46-57).

Next to age, several other demographic characteristics have been linked to differences in cognition and $\mathrm{EF}$ and should thus be included in the research reported here. Important ones are age, sex and level of parental education (Lange et al., 2006). The next demographic variable, level of parental education (LPE), can be seen as a proxy of socioeconomic status (e.g. White, 1982). Socio-economic status is a multi-component construct 
thought to reflect parental/caregiver's income, their profession and their level of education (Luria et al., 2009). Also, it is associated with variance in children's EFs development (Ardila et al., 2005; Klenberg et al., 2001), such that higher LPE is associated with children's higher EFs test scores. The LPE effect was consistently found across different age groups. Last, the demographic variable sex has been associated with differences in EFs (Ardila et al., 2011), but it remains much debated at the same time. Some researchers found (marginal) sex differences in EFs in children (Ardila et al., 2011; Brocki \& Bohlin, 2004 ), but others did not (Galsworthy et al., 2000). This supports the need to include the three demographic variables of age, LPE, and sex in the analyses in this study, since these may influence the outcomes in various ways. By including such confounding influences, norm scores appropriate for the sample under study can be derived that approximate as closely as possible the characteristics of individuals who belong to the population under investigation and thereby also the meaning of the interpretation of the scores (Strauss et al., 2006).

\subsection{The influence of context: Family and community in Ukraine}

Before commencing the second part of this research project, informal observations and discussions took place with teachers in a Ukrainian primary and middle school. In these conversations teachers pointed out areas of concern for development in the children's environment. Specifically, they expressed concerns about the availability of alcohol to young people and their alcohol consumption, as well as possible alcohol (mis)use in families. They also told about some of the strategies used by their school to prevent young people to start (mis)using alcohol. Such environmental characteristics may range from the people in a child's life to the food (vitamins) that is available, and these may have an (dis)advantageous influence on a child's development. Relevant to the current research, some of these influences are changeable with timely interventions, thereby reducing their possibly negative influence on the child. However, some contextual factors may (seemingly) be fixed; and the situation in Ukraine shows they sometimes have a background in society's history.

Ukraine has a long tradition and culture of (unrecorded) wine brewing, and wine consumption with family meals in the shade of vines (Popova et al., 2007). Furthermore, beer is worldwide one of the most consumed beverages and the preference of 11-15 years old adolescents (Currie, 2004; WHO, 2014). On top of that beer consumption in Ukraine has tripled between 2002-2009 (Goncharuk, 2009). In Ukraine 9.3\% of boys and 3.1\% of girls of 11 years old drink alcohol weekly (Currie, 2004). Chambers et al. (2014) found in their large sample of pregnant Ukrainian women, that $97 \%$ were ever drinkers and $46 \%$ of the pregnant women had consumed alcohol in the last month. Based on these figures, alcohol use - both by the adolescent and/or his immediate environment - can be perceived as a risk factor for child development in Ukraine (Popova, Lange, Shield, Burd, \& Rehm, 2019). It is therefore important to investigate what the perceptions of alcohol use are among adults and youths in West Ukraine. 


\section{Chapter 1}

Development arises from child characteristics (e.g., cognition, initiative, perseverance in for example pursuing learning in school; Zimmerman, 2001) and the interaction of the child with his/her environmental (e.g., bio-ecological model;

Bronfenbrenner \& Morris, 2006). Children's relations in the immediate home environment extend and increase once a child comes in contact with peers and teachers, into the community and then larger organizations such as high school, and so on (Bronfenbrenner \& Morris, 2006). In a disorganized home environment parents and children may need most attention for daily tasks, while parents in an organized family setting may have time and energy to teach children knowledge and skills, for example to cope with organizational challenges in the environment outside the family. In the latter situation the child may have learnt and be able to apply coping skills in situations, whereas the child from a disorganized home environment might not (Bronfenbrenner \& Morris, 2006). This example highlights possible differences in outcomes related to environmental factors. Since the teachers' main concern regarding children's (home) environment seemed to involve alcohol (mis)use, in the second part of this dissertation perceptions of alcohol use and possible alcohol dependence in Ukraine were explored using a qualitative approach. Alcohol misuse is included in this study to describe binge drinking ( $>4-5$ consumptions on one occasion for females and males respectively; Wechsler \& Kuo, 2000) or other forms of alcohol consumption that pose a risk to health such as underage drinking. Alcohol dependence is in this study not a diagnostic term as defined in the DSM-V (APA, 2013), but follows these descriptions (including alcohol use disorder). Such descriptions are e.g., levels of alcohol use interfere with daily activities in work- and social life and/or there is a need to consume alcohol.

In addition to the physical and psychiatric consequences of alcohol use, alcohol use disorder is a severely stigmatized condition worldwide (Schomerus et al., 2010). According to the original definition of Goffman (1997), stigma is a characteristic or an attribute of a person, which results in someone perceived as being of lesser value. Stigma processes have been linked to disadvantageous psychological, economic, and social consequences and stigmatizing a (member of a) group serves purposes of exploitation or norm reinforcement of the in-group (Crocker \& Major, 1989; Mak \& Cheung, 2010). Alcohol use disorder is often not perceived as a mental illness (compared to other (mental) illness), but it has instead been associated with a perceived character flaw of a person not being able to control oneself (Schomerus et al., 2010). This view implies that persons suffering from alcohol use disorder are responsible for the condition themselves, which provokes social rejection. Furthermore, alcohol dependence was associated with perceptions of being similarly dangerous as a person with schizophrenia (Schomerus et al., 2010). These perceptions may serve as justification to treat people as 'being of lesser value' through overt or covert behaviors, for example by limiting the stigmatized person's access to work (Pescosolido \& Martin, 2015). There are several types of stigma, each with their own determinants and consequences for the stigmatized individual.

Public stigma is defined as a general acceptance in society of the prejudiced views that exist about certain groups or individuals, and several other forms of stigma stem from 
this, i.e., stigma by association (e.g., a family member), self-stigma (i.e., internalizing the public perceptions), structural stigma (i.e., bodies or organization of society enhances the stigmatizing beliefs), and provider-stigma (i.e., staff in a hospital treat people with alcohol dependence differently; Pescosolido \& Martin, 2015). Ukraine falls in the highest WHO category of 'years of life lost' due to alcohol use, with an average alcohol consumption of 13.8 liters of pure alcohol per person per year (WHO, 2018). Recent research among young adults in Ukraine showed that especially those taking high risks in for example their occupation, reported more recurrent alcohol consumption (Polshkova, Chaban, \& Walton, 2016). These young adults furthermore reported to use alcohol to cope with negative affect (anxiety or stress), or as a result of social influence. Stigma linked to alcohol dependence (and/or alcohol use disorder as described by participants) of a person or their family in Ukraine therefore, may be an environmental risk factor and may affect young people from a parental background of alcohol dependence and/or alcohol use disorder. Perceptions concerning alcohol dependence in Ukraine were therefore also investigated. The last part of this general introduction is the outline of the dissertation.

\subsection{Outline of the dissertation}

To summarize, the studies ( 3 studies in part one and 2 studies in part 2) reported in this dissertation were conducted with two general aims in mind: The first aim was to establish regression-based normative scores for EFs and cognitive functions underlying successful EFs such as short-term memory, of children living in West Ukraine, corrected for demographic factors. In light of this aim, measurement of these EFs and related cognitive functions in 195 children aged 5.10 - 14.5 years old and normative data are reported and discussed in three studies in chapters $2,3 \& 4$ of this dissertation. The second aim was to explore perceptions of alcohol (mis)use and stigmatization surrounding alcohol dependence in Ukraine. To this end, qualitative research conducted (38 interviews with adults and 81 questionnaires with students 12-21 years old) which is reported and discussed in chapters 5 and 6.

Specifically, in chapter 2 the Delayed Matching to Sample, a test of 'visual matching ability and short-term visual recognition memory of non-verbalisable patterns' of the Cambridge Neuropsychological Test Automated Battery $\left(\mathrm{CANTAB}^{\circledR}\right.$; Cambridge Cognition, 2019) is discussed. Children from primary and middle schools participated in this task. Norms are calculated controlling for age, sex, and level of parental education. Outcome measures reported are the number of correct responses (accuracy), response time, and the probability of an error after an incorrect response.

In chapter 3, visual-, spatial- and visual spatial short-term memory (STM) is measured in schoolchildren in Ukraine using three CANTAB ${ }^{\circledR}$ tasks. The respective tasks are Pattern Recognition Memory (visual STM), Spatial Span (spatial STM) and Paired Associates Learning (visual spatial STM). Demographically corrected norms (age, sex and level of parental education) were established and presented for these three tasks. 


\section{Chapter 1}

Short-term memory is defined as the brief storage of a limited number of items (e.g., an object, a word, a digit, a location), and maintain these items for a short amount of time (possibly rehearsing it). This short maintenance of information in memory may then be followed by using this information (e.g., reproduction) such as recognizing e.g., a previously seen object among multiple presented objects (Majerus \& Van der Linden, 2013). This conceptualization finds support in Palmeri and Tarr's (2008) reasoning that perception of an object or pattern is closely related to memory processes, till the extent that the two can be viewed as inseparable processes, in which perception theoretically precedes short-term memory. Next, working memory is theorized to be used for the short retention of information and manipulation of that information in the service of complex cognitive behaviors such as solving a problem (Fuster, 2002). The latter appears aligned with Anderson's (2002) definition of working memory.

In chapter 4, goalsetting tasks in schoolchildren in Ukraine, i.e., the EF domains of goal setting and cognitive flexibility, are investigated (Anderson, 2002). Goal setting requires deriving new solutions, planning the steps to achieve those goals and executing these steps (Anderson, 2002). Also, working memory, as part of the executive domain of cognitive flexibility (see Figure 1), is needed for example to adapt a plan based on mistakes. In this chapter results are reported on two CANTAB ${ }^{\circledR}$ computerized tests (Spatial Working Memory and Stockings of Cambridge; Cambridge Cognition, 2012) and one paper-based matrices task; the Naglieri Nonverbal Ability Test (NNAT; Naglieri, 2003). The influence of the demographic variables age, sex and level of parental education on these test scores is evaluated.

In chapter 5 the results of the qualitative investigation (interviews and semistructured open-ended questionnaires) of perceptions about health and alcohol use in Western Ukraine are discussed. Data were collected in seven villages, four cities and one province capital in Western Ukraine. Multiple stakeholders were invited for semi-structured interviews ( $n=38$ adults) in order to describe alcohol use in detail from different perspectives; family of people misusing alcohol, community leaders, people recovered from alcohol misuse/dependence, people at high risk of continued alcohol misuse/dependence, health care professionals, European international volunteers (students doing one-year community work), and international professionals working part/full time in Ukraine. Additionally, to capture young Ukrainians' views on these issues, semi-structured openended questions (questionnaires) were administered with $n=81$ students aged 12-21 years old, currently enrolled in middle - and high schools and colleges. These semi-structured measures were used to make an inventory of perceptions of alcohol use in this sample of Ukrainians. The focus is on identifying differences and similarities between stakeholder groups such as youths and adults in Ukraine.

In the last empirical chapter, chapter 6 the specific theme of stigma surrounding alcohol dependence, that arose from the qualitative data and analysis presented in chapter 5 , is reported. The aim of this chapter is to investigate what the perceptions are in West Ukraine about people suffering from alcohol dependence and their family members and what possible consequences stigmatization has for the individual and their families. 
Finally, in chapter 7, an overview and summary of the main findings from the empirical chapters is provided. Findings are discussed in the context of the literature as well as methodological limitations in this dissertation on vulnerabilities in development of schoolchildren in Ukraine. Finally, suggestions for interventions that may contribute to a decrease in children's developmental vulnerabilities are proposed. 
Chapter 1 


\section{CHAPTER 2}

Measuring visual matching and short-term recognition memory with the CANTAB ${ }^{\circledR}$ Delayed Matching to Sample task in schoolchildren: Effects of demographic influences, multiple outcome measures and regression based normative data

Published as: Toornstra, A., Hurks, P. P. M., Van der Elst, W., Kok, G., \& Curfs, L. M. G. (2019). Measuring visual matching and short-term recognition memory with the CANTAB ${ }^{\circledR}$ Delayed Matching to Sample task in schoolchildren: Effects of demographic influences, multiple outcome measures and regression based normative data. Child Neuropsychology, 1-30. doi.org/10.1080/09297049.2019.1642316 


\title{
Chapter 2
}

\begin{abstract}
The study aims to establish demographically corrected, pediatric norms for the computerized Delayed Matching to Sample (DMS) test, a measure of "visual matching ability and short-term visual recognition memory, for non-verbalisable problems" (Cambridge Cognition, 2019). The DMS was administered to $n=184$ children aged 5.10 to 14.5 years old. The DMS is a 4-choice recognition task of non-verbal, abstract patterns. The child has "to select, among four different choice patterns, the one that matches a complex visual pattern presented" i.e., (the target stimulus) (Bersani et al., 2016). The DMS consists of two conditions: a) the overt condition in which the target stimulus and four choice patterns are shown simultaneously and b) the covert condition, in which the choice patterns are shown after the target pattern is covered. The DMS test provides three outcome measures: the accuracy score (i.e., the number of correct patterns selected) score, latency (i.e., the response speed) and the probability of making an error after an incorrect response. These outcome measures were calculated for both conditions and for both conditions combined. Results showed that demographic variables, such as age, sex, and/or level of parental education (LPE) affected scores on these outcome measures. Based on these data, demographically corrected norms were established for all outcome measures, per condition and for both conditions combined.
\end{abstract}

Keywords: Visual spatial memory; Delayed Matching to Sample (DMS); Demographic influences; Continuous norms; schoolchildren; Ukraine 


\section{Introduction}

The Delayed Matching to Sample (DMS) task, which is part of the Cambridge Neuropsychological Test Battery (CANTAB®) Cambridge Cognition, 2012) is used worldwide in clinics and research. The task is believed to be a "measure of simultaneous matching ability and short-term visual recognition memory, for non-verbalisable problems" (Cambridge Cognition, 2019; Hammers et al., 2011; Smith, Need, Cirulli, Chibak-Falek, \& Attix, 2013). DMS test results however, are only meaningful when compared to suitable norm data for a sample of schoolchildren. In the current study, DMS normative data were prepared for children living in Ukraine. The DMS offers users multiple outcome measures: i.e., accuracy scores (which is the number of correct patterns selected), the response latency (i.e., the response speed), and the statistical probability of making an error after an incorrect response. Normative data were collected here for all DMS outcome measures. The DMS test, norming procedures, and results are discussed next.

The DMS task is a 4-choice recognition task of non-verbal abstract patterns. The task consists of 20 trials. In each trial, the child has "to select, among four different choice patterns, the one that matches a complex visual pattern presented (i.e., the target stimulus; see Figure 1 for an example) (Bersani et al., 2016, see also Lecerf \& Ribaupierre, 2005; Cambridge Cognition, 2012). Furthermore, inhibition is needed to be able to perform well on 4-choice recognition tasks, such as the DMS task (Davidson, Amso, Anderson, \& Diamond, 2006). The DMS consists of two conditions: an overt condition (i.e., the target stimulus and the four choices are shown simultaneously) and a covert condition (i.e., the four choices are shown after the target is covered). The DMS overt condition is thought to be more a measure of "simultaneous visual matching abilities" (Mammarella, Pazzaglia, \& Cornoldi, 2008) i.e., the target stimulus needs to be recognized, but four choices can be directly checked against the target. The DMS covert condition is thought to be associated with spatial-sequential memory processes, i.e., the covering of the target requires the maintenance of the abstract pattern in visual memory (Mammarella et al., 2006;

Mammarella et al., 2008).
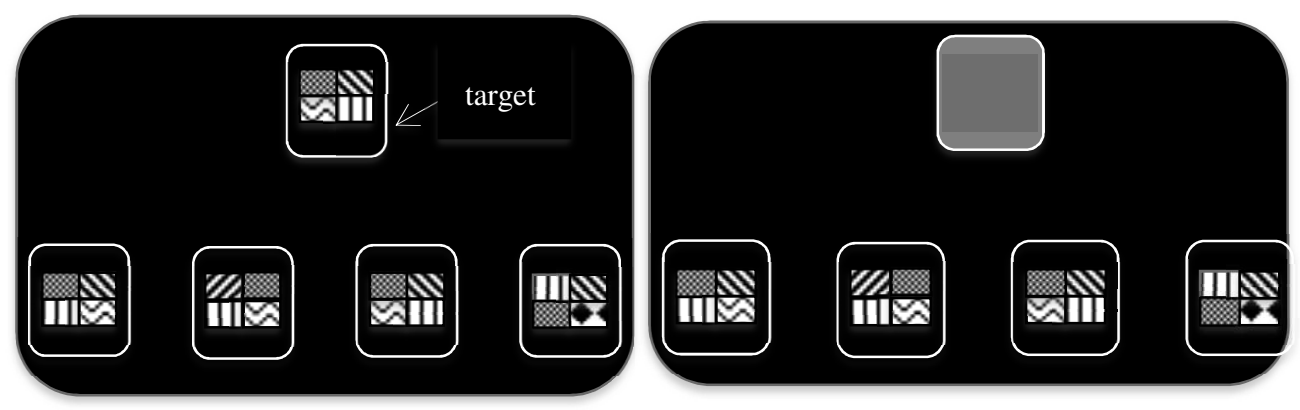

Figure 1. DMS on a touch screen: Simultaneous view of target and stimuli (Option 3 is identical to target), and covert (delayed) target (this is not a real CANTAB ${ }^{\circledR}$ DMS sample). 


\section{Chapter 2}

As mentioned earlier, the DMS test results are only meaningful when compared to fitting norm data (Mitrushina, Boone, Razani, \& D'Elia, 2005, p. 3). Even though DMS normative data have been published for schoolchildren living in several countries such as America and Finland (e.g., Luciana \& Nelson, 2002; Green et al., 2019; Lehto, Juujärvi, Kooistra, \& Pulkkinen, 2003; Roque et al., 2011; Vinţan et al., 2012) this is not the case for an Ukrainian pediatric sample. Culture affects thinking, knowledge, values and beliefs (Ardila, 2006), which are shaped in social situations. Cultural variances are linked to geographical and ethnic differences. The $\mathrm{CANTAB}^{\circledR}$ norms have been mainly established in Western (European and North American) societies. In line with Frazier Norbury and Sparks' (2012) reasoning, analyzing data and proposing norms for other populations contributes to refinement of current culturally influenced criteria for tests. Analyzing test scores of non-Western samples may contribute to refinement of cognitive frameworks currently used and the understanding of how to distinguish typical from atypical development. The aim of the present study was to establish a normative data range on the DMS test, using a sample of schoolchildren in Ukraine.

In addition, norm data currently available worldwide for the DMS include limitations. For one, these norm data are almost always calculated based on means and standard deviations for relevant sub groups of children separately, e.g., per two-year age band. This method of collecting norm data has significant limitations (see e.g., Van der Elst, Hurks, Wassenberg, Meijs, \& Jolles, 2011). For instance, multiple studies have shown that performances on numerous cognitive tests are influenced by the demographic variable 'age' (Lezak, Howieson, Bigler \& Tranel, 2012). In establishing normative data the total sample tends to be subdivided into many different sub groups based on e.g., age. Some disadvantages of making these sub groups in the data are that test scores are only applied to a specific (age) sub group in the sample, and children closely in age (i.e., 1 month apart) may be in different (age) sub groups. Additionally, the (age) sub groups themselves may show unusual characteristics, yet result in a norm for that (age) sub group.

Also, so far we are only including one demographic variable in our example, i.e., age, whereas other variables such as sex and/or level of parental education are believed to influence test performance on cognitive tests, such as the DMS, as well (Strauss, Sherman $\&$ Spreen, 2006). We will apply a promising method, called continuous norming here, which offers the option to include multiple demographic variables simultaneously, while establishing normative data (in line with an approach suggested by Van Breukelen \& Vlaeyen, 2005; Van der Elst et al., 2011). This approach to norming (Van Breukelen \& Vlaeyen, 2005) is a regression-based method, which allows including both continuous variables (e.g., age) and categorical variables (e.g., sex and/or level of parental education (LPE)), without creating the sub groups for each variable (Van der Elst et al., 2011). In doing so, the relation between age and the test score to-be normed is estimated based on all data points in the sample. This allows for more adequate estimates of age-related normative scores (Bechger, Hemker, \& Maris, 2009). Additionally, the option of incorporating more than one demographic characteristic in regression models makes this normative method rather accurate (Van der Elst et al., 2011). 
The demographic variables of age, sex and LPE are incorporated in this study because these have been associated with differences in cognitive test performance (Strauss et al., 2006), and partly with DMS scores more specifically. For one, performance on the DMS test has been shown to improve with age, i.e., from early childhood well into the middle school years (Huang, Klein, \& Leung, 2016; Perlman, Huppert, \& Luna, 2015). Studies including typically developing children using the DMS paradigm have shown that this test can be administered in children as young as 5 years and older and that the cognitive functions measured with the DMS develop rapidly in young children and mature in adolescence (Green et al., 2019; Paule et al., 1998; Roque, Teixeira, Zachi, \& Ventura, 2011; Luciana, 2003; Luciana \& Nelson, 1998, 2002 - see below for more details on developmental trajectories of DMS scores). Further support for content validity (i.e., the test measures what is said to be measured) is found in for instance, Green and colleagues (2019) who administered the DMS in a group of 5-15 years old children from Mexico City. Green et al. (2019) found in line with the above researchers that DMS scores continue to improve (linearly) into adolescence, i.e., up until age 15 years old. They found for instance, that children aged 5 produced on average approximately $60 \%$ correct solutions on the DMS test, whereas children e.g., aged 7 scored approximately $74 \%$ correct solutions. These authors only studied "the percentage of correct solutions" (accuracy) calculated over all items, Also to the best of our knowledge, the majority of the studies on age-related effects in DMS scores reported only on the total accuracy scores without the separate conditions (overt vs covert) or latencies.

Data on the relation between sex differences in performances on the DMS task, or tasks measuring constructs similar to those measured with the DMS task such as visual memory, are still inconclusive: several studies (e.g., Luciana \& Nelson, 1998; Green et al., 2019) did not find any sex differences on the DMS total accuracy in children. However, León, Cimadevilla, and Tascón (2014) proposed a developmental view on sex differences in visual memory in childhood. The demographic variable, 'level of parental education' (LPE), has not yet been studied in relation to the DMS in schoolchildren. It is perceived as an estimate of socio-economic status (SES; White, 1982) and higher levels of either have been positively linked to children's higher academic achievement (Davis-Kean, 2005) and cognitive functions such as memory (Kaplan et al., 2001; Noble, McCandliss, \& Farah, 2007).

This study is furthermore unique in reporting norm data on multiple outcome measures for the DMS test, as mentioned above. Traditionally, paper and pencil cognitive tasks, such as the Knox Block test, which is believed to be a measure of visual memory (Richardson, 2005), report only the total number of correct remembered responses (i.e., a measure of accuracy). The DMS offers the opportunity to not only include a measure of accuracy but also of e.g.,response latency (i.e., the response time from the moment the response items are shown). The few pediatric studies focusing on both accuracy and response latency on the DMS and/or on other cognitive tasks showed the relevance of collecting norm data for both. For one, Chien et al. (2015) reported accuracy and response latency measures in a sample of 143 youths with ASD and controls (mean age 13 years old, 


\section{Chapter 2}

sd 3.5). They found that accuracy scores, and not response latencies differed between the control and clinical group of participants on the DMS. Vice versa, Wassenberg et al. (2008) found that, compared to healthy control children, response latencies were longer (i.e., they reacted slower) in children with ADHD on a cognitive task other than the DMS. However these groups did not differ in accuracy on the same task. Thus, children with ADHD were able to perform the task accurately, but it took them more time than others, which has implications for example for interventions. These findings warrant a separate analysis (and separate norm data) of both accuracy and reaction time outcome measures on the DMS.

In sum, the aim of the study was to establish a demographically corrected normative data range on multiple outcome measures of the DMS test, using a sample of $n=$ 184 school children in rural Ukraine, age 5.10 to 14.5 years old.

\section{Methods}

\section{Participants}

The sample consisted of $n=184$ children enrolled in the local primary and middle schools in the Ukraine. Per school all children were invited and 80,5\% participated. Characteristics of this sample are summarized in Table 1 (there were approximately equal numbers at different points across the age range except for children below 5.9 and above 14 years old). The level of parental education was based on the level of education completed by the parent(s) in a household; low described education up till grade 9 (i.e., primary and middle school) and high applied to grade 10 (high school) and above, in line with the Ukrainian educational system (Ukraine Channel, 2017). In the few cases of a discrepancy between parent's (or caregiver's) level of education (6.5\%), the highest level was allocated as the mean for the household (in line with e.g., Jiang, Ekono, \& Skinner, 2016). Parental level of education and the kind of schooling they could afford in the Ukraine has been linked to children's test scores (Ardila, Rosselli, Matute, \& Guajardo, 2005; Jiang et al., 2016). For the purpose of representativeness of the study sample, a similar number of children was included per age group. For all age groups we succeeded, except for the oldest and youngest age groups $5.5-5.9$ years old and $14.5-14.9$ years old. The sample sizes of these latter groups were unfortunately smaller in size, however note that age was included as a continuous variable ranging from $5.10-14.5$ years old in the analyses. 
Table 1: Basic demographic data

\begin{tabular}{|l|l|l|l|l|l|l|l|}
\hline Age range & $N$ & $\begin{array}{l}\text { Age } \\
\text { (Mean in } \\
\text { years) }\end{array}$ & $\begin{array}{l}\text { Age (SD of } \\
\text { mean) }\end{array}$ & \multicolumn{2}{|l|}{ Gender } & \multicolumn{2}{|l|}{$\begin{array}{l}\text { Level of Parental } \\
\text { Education }\end{array}$} \\
\cline { 4 - 8 } & & & Male & Female & High & Low \\
\hline Total Sample & 184 & 9.61 & 2.49 & 89 & 95 & 161 & 23 \\
\hline $5.10-7.92$ & 60 & 6.8 & 0.63 & 29 & 31 & 49 & 11 \\
\hline $8-10.92$ & 66 & 11.1 & 0.87 & 34 & 32 & 57 & 9 \\
\hline $11-14.5$ & 58 & 12.67 & 1,02 & 26 & 32 & 55 & 3 \\
\hline
\end{tabular}

\section{Procedure and instrument}

The researchers approached schools to gauge interest for participation in the study, after which the school management invited parents to the information meetings about it. Information and consent letters were provided by the researchers and school management and then taken home. The testing started once parents and children had given informed consent letters to their teachers. The research ethics committee of the Faculty of Psychology and Neuroscience of Maastricht University, The Netherlands, approved this study. All data were obtained in compliance with the ethics regulations of the WMA declaration of Helsinki. Accordingly, debriefing was provided in a personal report to each student (parent/guardian) at the individual level, and anonymous class- and school reports were provided to school management. Testing took place during school hours without a need for compensation.

The Delayed Matching to Sample (DMS, Cambridge Cognition, 2012), was administered individually in a separate room on a HP Pavilion TS sleek-book laptop with a 15-inch touch screen by two certified researchers. Standard administration procedures prescribed by CANTAB ${ }^{\circledR}$ were followed. The test included both practice trials and test trials in which the child had to indicate, from four choices, the one pattern that is identical to the target. Per trial the child received direct feedback (i.e., correct/incorrect response). Per trial, the four choices were either shown simultaneously (i.e., the overt condition, $n=5$ trials) or after the target was covert (i.e., the covert condition, $n=15$ trials (i.e., the practice trials were additional). During the practice trials the child is asked to perform trials from both conditions starting with an overt and followed by two covert trials with varying delays. The 20 trials (excluding the practice trials) are randomized and fixed. Touching the screen anywhere except for the correct response item is not recorded. Administration time is about 8 minutes (Cambridge Cognition, 2012).

The outcome measures of the DMS test can be divided in three groups; accuracy, response latency, and probability of an error after an incorrect response. The accuracy 


\section{Chapter 2}

scores for the DMS (i.e., the total amount of correct responses in all conditions on the first attempt, reported here as DMS accuracy), were reported for the combined conditions (total) as well as per condition, i.e., the overt and covert conditions separately. Response latency was measured in milliseconds (ms) and refers to the time from the appearance of response items to the selection of the pattern that is identical to the target pattern. Mean latencies are reported over all times and for the overt and covert conditions separately. The probability of making an error after an incorrect response indicates participant's sensitivity to negative feedback and is not reported per condition (given that there are not sufficient trials). This measure is based on signal detection theory (SDT; Goldstein, 2002) and ranges from 0 (highest) to 1 (i.e., an increased chance of making errors). In the DMS the participant received feedback (incorrect response results in a red line around the response item) and is required immediately after completing the trial, to process the next trial (target stimulus). Based on Bayes' theorem this probability is calculated by adding failing on problem 1 and subsequently on problem 2 , which is divided by the total amount of errors made by the participant (Elliott et al., 1996). An optimal response is thought to reflect the participant's sensitivity to the stimulus, i.e., identifying the correct response item after presentation of the target irrespective of noise (among others a previously made incorrect response; Goldstein, 2002). A high score indicates a high tendency to make an error after an error (Cambridge Cognition, 2012) and has been associated with e.g., depression (Elliott et al., 1996), which once more supports the need to propose suitable norms for a sample.

\section{Statistical analyses}

The exploratory analysis, started with providing an overview of the descriptive data, i.e., the means and standard deviations for the main outcome measures (Table 2). Next Pearson correlations among all the DMS outcome measures (including the total scores and those calculated for the overt and covert conditions separately) were computed (see Table 3). Furthermore, a summary independent sample $t$-test was used to compare the observed means of the DMS accuracy, response latency and probability of an error after an incorrect scores of our Ukrainian sample to the Western CANTAB ${ }^{\circledR}$ mean child scores $\left(\right.$ CANTAB $^{\circledR}$ child norms, Cambridge Cognition, 2014). This comparison was based on the means of the total scores of children $6-13$ years old in the respective samples (i.e., the ages that overlapped between the two samples). This summary independent sample $t$-test is based on the overall means of the respective age groups of each sample and the mean of the standard deviations (Field, 2009).

Next, the effects of the demographic variables on these DMS scores were further analyzed with multiple linear regression analyses. The full regression models included age, age $^{2}$, sex and LPE and all two-way interactions as predictors. Age was centered (Age_C = calendar age in months - mean age of the sample, which equaled 115.36 months) before computing the quadratic age variable to avoid multi-collinearity (Van der Elst, van Boxtel, van Breukelen, \& Jolles, 2005). Sex was dummy coded as $0=$ female and $1=$ male. LPE was dummy coded as $0=$ low and $1=$ high. Next to the total (i.e., the overt and covert 
conditions combined) DMS scores for accuracy and response latencies, we analyzed the DMS scores on the overt and covert conditions separately. DMS scores on the covert condition were analyzed using multiple linear regression analyses (similar to the procedures mentioned for the other DMS scores). Scores on the DMS accuracy overt (simultaneous) condition were analyzed using an ordinal logistic regression analysis with cumulative probability due to the restriction in the range of possible scores (0-5 items; Laerd Statistics, 2015). The same demographic variables as for the other DMS analyses were used.

All final regression models were achieved in a step-down hierarchical procedure by excluding the non-significant predictors from the model. The assumptions of regression analysis were tested separately per model. Importantly, regressions require a normal distribution of the residuals only (Field, 2009) and not for the raw test scores. Meeting these assumptions of the normal distribution of the residuals was therefore tested. The normal distribution of the residuals was tested with Kolmogorov-Smirnov tests on the residual values. The predicted values were grouped in quartiles. The Levene test of the standardized residuals based on these quartile groups tested for homoscedasticity (Van der Elst et al., 2011). Multi-collinearity was assessed using the Variance Inflation Factors (VIF, which should be below 10), and influential cases by calculating Cook's distances (Fisher et al., 2014).

Next, normative data, based on the final regression models (i.e., accuracy, overt and covert conditions respectively, including response latencies), were calculated using four steps (Van Breukelen \& Vlaeyen, 2005; Van der Elst et al., 2005). First, the expected test scores were computed applying the regression model $(=\mathrm{B} 0+\mathrm{B} 1 \mathrm{X} 1+\ldots+\mathrm{BnXn}$, with $\mathrm{B} 0=$ the intercept, $\mathrm{B} 1, \ldots \mathrm{Bn}$ the regression weights for the demographic variables and $\mathrm{X} 1$, ... Xn the values of the demographic variables). Then the residuals were calculated (= observed score - expected score). In the next step the residuals were standardized using the standard deviation (SD) of the residuals in the normative sample (=residual /SD (residual) of the normative sample) (Van der Elst et al., 2011). In the last step the residuals were converted into percentile values following the standard normal distribution if the assumption for normality of the standardized residuals was met in the normative sample. The appendix shows these converted scores. An alpha level of .01 was applied to avoid Type 1 errors due to multiplicity. All calculations were carried out in SPSS version 24. The norm calculations for DMS accuracy overt followed the described procedure but are limited due to the range of $0-5$ (results and assumptions reported below). The overt accuracy scores therefore allowed comparison to the covert and total accuracy but likely show ceiling effects in observed and thus predicted scores, which are summarized in a cumulative norm table (see Appendix).

\section{Results}

The means and standard deviations for all DMS outcome measures were calculated for the Ukrainian sample (Table 2). 
Chapter 2

Table 2: Mean and standard deviation of all DMS outcome measures

\begin{tabular}{|l|l|l|l|}
\hline & $N$ & Mean & Standard Deviation \\
\hline DMS Accuracy (total) & 184 & 14.27 & 3.45 \\
\hline DMS Accuracy Overt (Simultaneous) & 184 & 4.46 & 0.91 \\
\hline DMS Accuracy Covert (All Delays) & 184 & 9.81 & 2.91 \\
\hline DMS mean Response Latency (accuracy total) & 184 & 4217.14 & 1174.91 \\
\hline $\begin{array}{l}\text { DMS mean Response Latency accuracy overt } \\
\text { (simultaneous) }\end{array}$ & 182 & 4016.00 & 1222.80 \\
\hline $\begin{array}{l}\text { DMS mean Response Latency accuracy covert (All } \\
\text { delays) }\end{array}$ & 184 & 4296.62 & 1365.45 \\
\hline DMS Probability of an error after an incorrect response & 179 & 0.27 & 0.24 \\
\hline
\end{tabular}

All children completed the practice trials of the DMS successfully. The summary independent sample $t$-test comparing the overall means of the respective age group means (6 - 13 years old) of our Ukrainian sample $(n=156$, mean accuracy 13.98; mean latency 4241.02 , mean probability of error $n=153,0.27)$ to the Western CANTAB $^{\circledR}$ age groups groups ( $n=72$, mean accuracy 14.84 , mean latency 4107.25 , mean probability of error $n=$ $69,0.21)$ appeared to show no significant difference between the two samples. The DMS outcome measures were based on the total mean scores (DMS Accuracy $t(53.5)=-0.31, p=$ 0.76 ; DMS response latency $t(53.5)=0.09, p=0.93$; DMS probability of an error after an incorrect response $t(53.25)=-0.37, p=0.71)$ and overlapping $95 \%$ confidence intervals. Figures $2,3 \& 4$ show the age-based group means of the Ukrainian and CANTAB ${ }^{\circledR}$ sample (including the overt and covert conditions separately for accuracy and response latencies). 


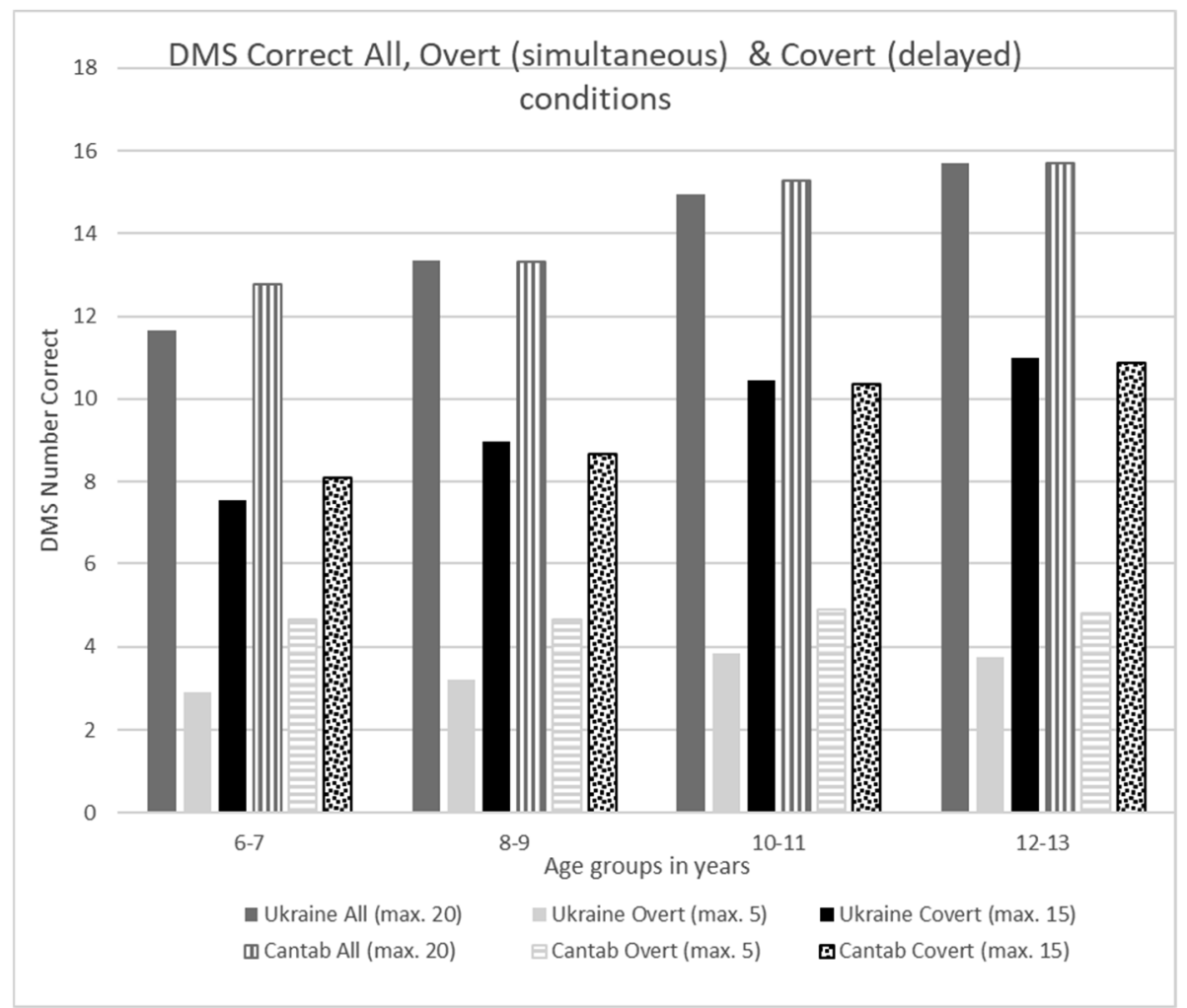

Figure 2. Observed scores for the mean DMS accuracy (i.e., total, overt and covert conditions) Ukrainian sample to the $\mathrm{CANTAB}{ }^{\circledR}$ traditional mean norms (2-year age groups). 
Chapter 2

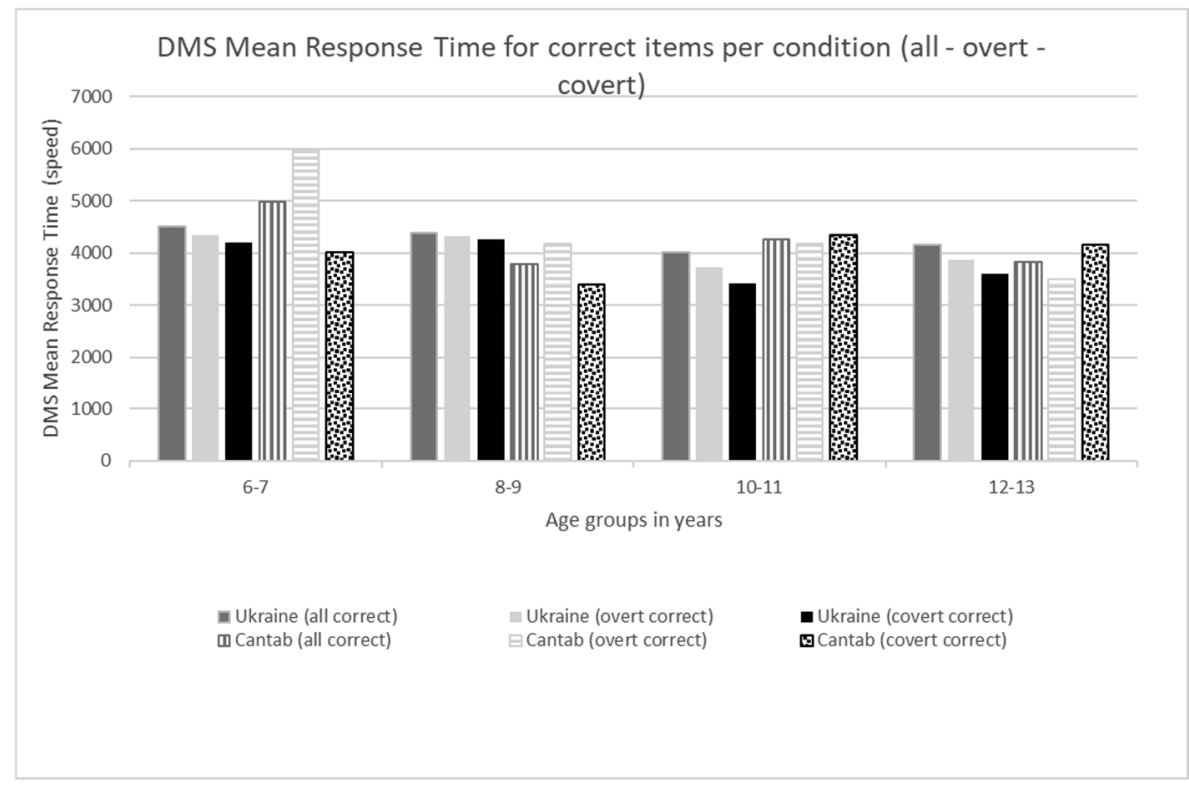

Figure 3. Observed scores for the mean DMS response latency for accuracy scores (i.e., total correct, overt correct and covert correct conditions), comparing the Ukrainian sample to the CANTAB ${ }^{\circledR}$ traditional mean norms (2-year age groups).

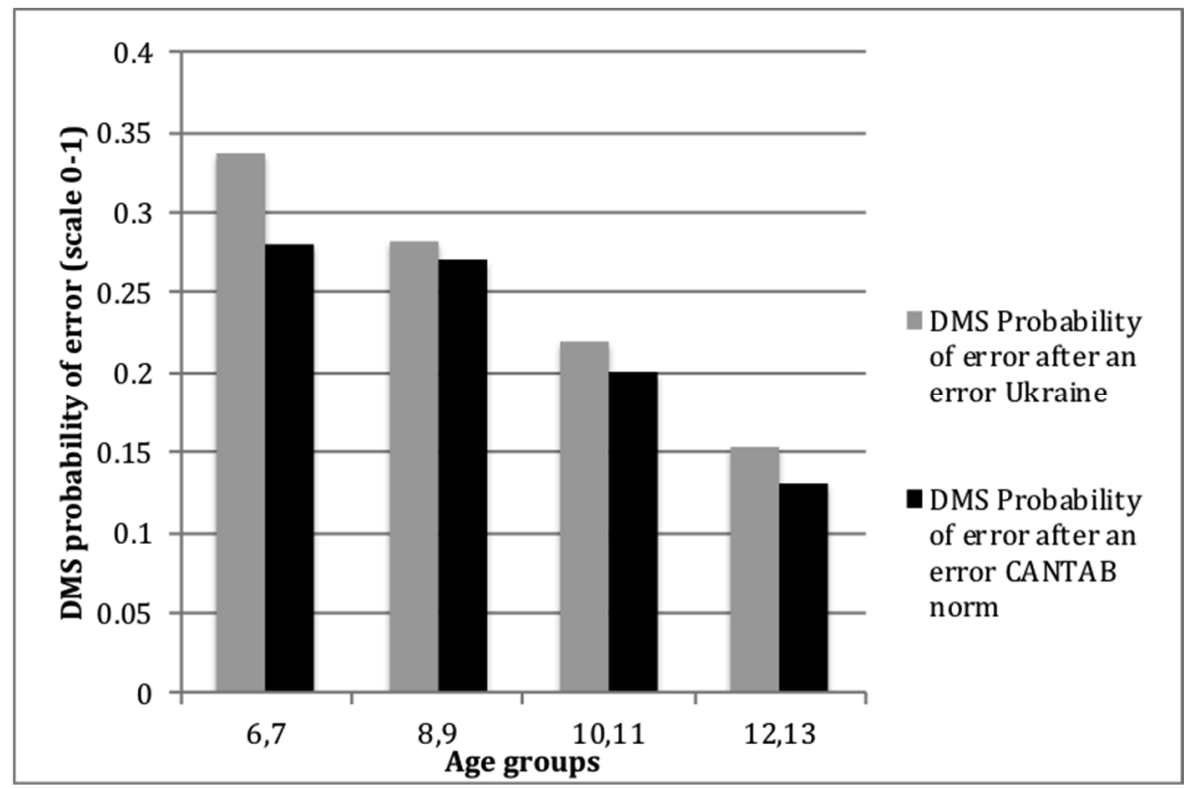

Figure 4. Observed scores for the mean DMS probability of error after an incorrect response comparing the Ukrainian sample to the CANTAB ${ }^{\circledR}$ traditionally mean norms (2-year age groups). 
Table 3 shows the correlations between the DMS outcome measures. In principle, correlations indicated that different latencies (total, overt and covert) correlated among each other. The same holds for the different accuracy measures. In contrast, response latencies and accuracy showed no meaningful relationship. Probability of an error after an incorrect response only correlated with accuracy measures.

Table 3: Pearson zero order correlations for DMS accuracy, response latency, and probability of an error after an incorrect response variables

\begin{tabular}{|c|c|c|c|c|c|c|c|c|c|}
\hline & & $\mathrm{N}$ & 1 & 2 & 3 & 4 & 5 & 6 & 7 \\
\hline 1 & $\begin{array}{l}\text { DMS ACCURACY } \\
\text { (TOTAL) }\end{array}$ & 184 & 1 & & & & & & \\
\hline 2 & $\begin{array}{l}\text { DMS ACCURACY } \\
\text { OVERT } \\
\text { (SIMULTANEOUS) }\end{array}$ & 184 & $0,68 * *$ & 1 & & & & & \\
\hline 3 & $\begin{array}{l}\text { DMS ACCURACY } \\
\text { COVERT (ALL DELAYS) }\end{array}$ & 184 & $0,97 * *$ & $0,49 * *$ & 1 & & & & \\
\hline 4 & $\begin{array}{l}\text { DMS MEAN RESPONSE } \\
\text { LATENCY (ACCURACY } \\
\text { TOTAL) }\end{array}$ & 184 & 0,08 & $0,15^{*}$ & 0,05 & 1 & & & \\
\hline 5 & $\begin{array}{l}\text { DMS MEAN RESPONSE } \\
\text { LATENCY ACCURACY } \\
\text { OVERT } \\
\text { (SIMULTANEOUS) }\end{array}$ & 182 & $-0,02$ & 0,14 & $-0,06$ & $\begin{array}{l}0,75 \\
* *\end{array}$ & 1 & & \\
\hline 6 & $\begin{array}{l}\text { DMS MEAN RESPONSE } \\
\text { LATENCY ACCURACY } \\
\text { COVERT (ALL DELAYS) }\end{array}$ & 184 & 0,10 & 0,14 & 0,08 & $\begin{array}{l}0,95 \\
* *\end{array}$ & $0,50 * *$ & 1 & \\
\hline 7 & $\begin{array}{l}\text { DMS PROBABILITY OF } \\
\text { AN ERROR AFTER AN } \\
\text { INCORRECT RESPONSE }\end{array}$ & $\begin{array}{l}179 \\
* * *\end{array}$ & $-0,65$ & $-\overline{-}$ & $-\overline{-}, 59 * *$ & - & 0,01 & - & 1 \\
\hline
\end{tabular}

** CORRELATION IS SignificANT AT THE 0.01 LEVEL (2-TAILED).

* Correlation is Significant At the 0.05 LeVel (2-TAiled).

*** N IS LOWER DUE TO PARTICIPANTS NOT HAVING A SCORE I.E. NO ERRORS WOULD RESULT IN NO OPTIONS TO MAKE AN INCORRECT RESPONSE AFTER INCORRECT RESPONSE. 


\section{Results of the multiple regressions for the three outcome measures per DMS} condition

Next, the final multiple linear regression models that were significant are shown in Table 4. Power transformations, using the Box-Cox transformations, which ensured consistency across variables, were applied (i.e., the DMS accuracy total, DMS response latency overt and covert conditions), because preliminary analyses suggested heteroscedasticity with the untransformed scores (Osborne, 2010). The other outcome measures, i.e., the DMS accuracy overt and covert conditions, DMS response latency (for accuracy in total), and probability of an error after an incorrect response, were not transformed. Also, all DMS response latency scores displayed outliers (residual $S D>3$ of the mean and thus outside the normal distribution), which were removed. After these transformations and removal of outliers, the assumptions of multiple linear regression analyses were met for the final models of the DMS accuracy and latency i.e., KolmogorovSmirnov values $p \geq .02$. For the probability of an error score, the Kolmogorov-Smirnov value was $p \leq .001$ and therefore the normality assumption was violated. Following Van der Elst, Van Boxtel, Van Breukelen and Jolles (2008), this was accounted for in the construction of the norms for the probability of an error after an incorrect response scores by using the empirical distribution of the standardized residuals rather than the theoretical standard normal distribution). The other assumptions of the multiple regression models were met for the final models of all scores, i.e., all values of the Levene's statistic were $p \geq$ .02 ; Cook's distance values $<.01$; all Variance Inflation Factors $\leq 1.1$. None of the interaction terms reached significance. Also, the raw scores showed that $2.72 \%$ of the children achieved the maximum score on the DMS accuracy and $3.80 \%$ of the sample made only one mistake.

Table 4: Final regression models for the DMS accuracy (all conditions), response latency and probability of an incorrect response after an incorrect response.

\begin{tabular}{|l|l|l|l|l|l|l|l|l|}
\hline Test score & $\mathrm{n}$ & Variable & $\mathrm{B}$ & $\mathrm{SE}$ B & $\begin{array}{l}\text { Std. } \\
\mathrm{B}\end{array}$ & $\mathrm{t}$ & $\begin{array}{l}\text { SD } \\
\text { (residual) }\end{array}$ & $\mathrm{R}^{2}$ \\
\hline $\begin{array}{l}\text { DMS Accuracy } \\
\text { (total; Lambda } \\
0.5)\end{array}$ & $179 * *$ & (Constant) & 4.93 & 0.17 & & 28.90 & & \\
\cline { 2 - 9 } & & Age_C & 0.01 & 0.00 & .49 & 7.50 & & $.27^{*}$ \\
\hline $\begin{array}{l}\text { DMS Accuracy } \\
\text { Overt } \\
\text { (Simultaneous) }\end{array}$ & 184 & LPE & 0.42 & 0.18 & .15 & 2,31 & 0.75 & \\
\hline
\end{tabular}




\begin{tabular}{|c|c|c|c|c|c|c|c|c|}
\hline & & Age_C & 0.02 & 0.01 & 0.98 & $\begin{array}{l}\chi^{2}(1) \\
= \\
8.84\end{array}$ & 0.51 & $.03 *$ \\
\hline \multirow{4}{*}{$\begin{array}{l}\text { DMS Accuracy } \\
\text { Covert (All } \\
\text { Delays) }\end{array}$} & $183^{* *}$ & (Constant) & 8.81 & 0.54 & & 16.2 & & \\
\hline & & Age_C & 0.05 & 0.01 & 0.51 & 7.78 & & \\
\hline & & Age_C ${ }^{2}$ & -.001 & 0.00 & -0.16 & -2.46 & & \\
\hline & & LPE & 1.76 & 0.54 & 0.21 & 3.27 & 2.36 & $.30 *$ \\
\hline \multirow{2}{*}{$\begin{array}{l}\text { DMS Mean } \\
\text { Response } \\
\text { Latency } \\
\text { (accuracy total) }\end{array}$} & $180^{* *}$ & (Constant) & 4428.11 & 104.05 & & 42.56 & & \\
\hline & & Sex & -610.77 & 150.53 & -.29 & -4.06 & 1005.98 & $.09 *$ \\
\hline \multirow{3}{*}{$\begin{array}{l}\text { DMS Mean } \\
\text { Response } \\
\text { Latency } \\
\text { Accuracy Overt } \\
\text { (Simultaneous); } \\
\text { Lambda } 0.6\end{array}$} & $180^{* *}$ & (Constant) & 244.45 & 4.07 & & 60.09 & & \\
\hline & & Age_C & -0.24 & 0.1 & -0.18 & -2.45 & & \\
\hline & & Sex & -16.62 & 5.85 & -0.21 & -2.84 & 39.01 & $.06^{*}$ \\
\hline \multirow{2}{*}{$\begin{array}{l}\text { DMS Mean } \\
\text { Response } \\
\text { Latency } \\
\text { Accuracy } \\
\text { Covert (All } \\
\text { Delays); } \\
\text { Lambda } 0.58\end{array}$} & $183^{* *}$ & (Constant) & 163.24 & 5.04 & & 32.41 & & \\
\hline & & Sex & -23.52 & 7.26 & -0.23 & -3.24 & 48.96 & $.05 *$ \\
\hline \multirow{4}{*}{$\begin{array}{l}\text { DMS } \\
\text { Probability of } \\
\text { an error after an } \\
\text { incorrect } \\
\text { response }\end{array}$} & $176^{* * *}$ & (Constant) & .34 & .046 & & 7.26 & & \\
\hline & & Age_C & -.003 & .001 & -.36 & -4.88 & & \\
\hline & & Age_C ${ }^{2}$ & .00004 & .000 & .17 & 2.27 & & \\
\hline & & LPE & -.14 & .046 & -.21 & -3.01 & .20 & $.18 *$ \\
\hline
\end{tabular}

Note. Encoding of the predictors: Age_C $=$ Calendar Age in months - Mean Calendar Age in months 115.36, LPE (level of parental education). ${ }^{*} p \leq .02$.

$* * \mathrm{~N}$ is lower due to removal of outliers (> $3 \mathrm{SD})$. 


\section{Chapter 2}

*** $\mathrm{N}$ is lower due to participants not having a score i.e. no errors would result in no options to make an error after an incorrect response and removal of 3 outliers (> $3 \mathrm{SD})$.

\section{Results of the logistic regression for DMS accuracy in the overt condition}

A logistic regression with proportional odds assumption was run to determine the effect of age, age ${ }^{2}$, sex and LPE on the DMS accuracy overt condition. Two children scored 0 and one child scored 1 , so these scores were incorporated in the two-correct category, resulting in one group of 10 participants (for the lowest scores) for this analysis. Sex and LPE were not significant in this analysis. Proportional odds were assessed and a full likelihood ratio test comparing the fitted model (including the demographic variable age) to a model with varying location parameters, was below the significance threshold, $\chi^{2}(2)=$ $6.36, p=.04$. As this $p$ value for this model was below .05 separate binomial regressions were run for each category ( $=5$ correct, 4 correct, 3 correct, and 0,1,2 correct) showing similar odd ratios for $B$ for age (range .97 to 1.02), thus meeting the assumption of proportional odds. The deviance goodness-of-fit test showed that the logistic regression with proportional odds was a good fit to the observed data, $\chi^{2}(260)=172.49, p=.66$, but $64.8 \%$ of cells showed zero frequencies, which may indicate limitations in the model for predicting scores. Nonetheless, the final model fitted the data significantly better compared to an intercept-only model, $\chi^{2}(1)=9.56, p<.00$. The high zero cell frequently may be linked to the continuous nature of the independent variable (age), i.e., a characteristic of the analysis and thus of limited concern while the noted significant of the model appears in line with the observed data (Laerd Statistics, 2015). The odds of scoring 4 correct in the DMS accuracy overt condition were $12.95,95 \%$ CI $[7.44,22.56], \chi^{2}(1)=81.89, p=.00$. An increase in age (centered and expressed in months) was associated with an increase in the odds of DMS accuracy overt, with an odds ratio of $0.98,95 \%$ CI $[0.97,0.99], \chi^{2}(1)=8.84$, $p=.00$ (see also Figures $5 \& 2$ ). 


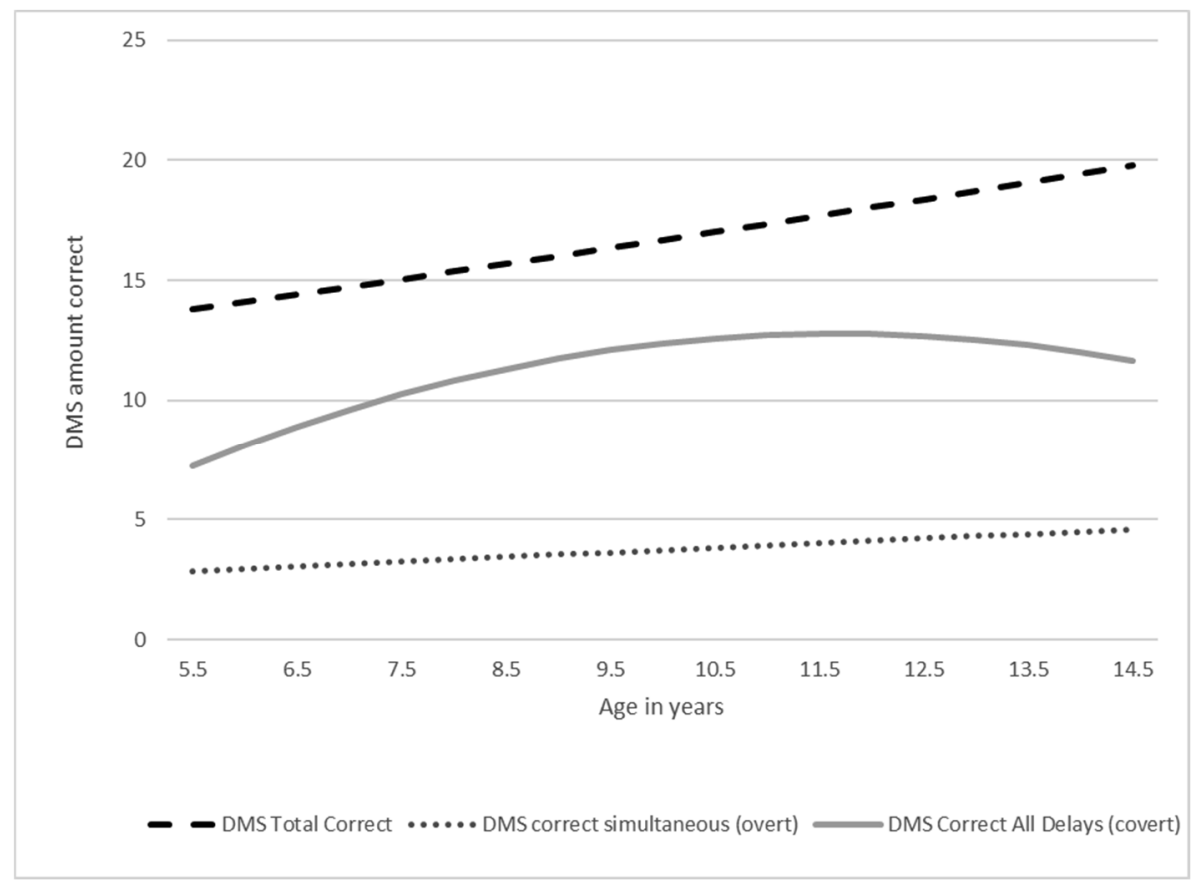

Figure 5. Expected test scores based on the regression models for the DMS accuracy (total correct), accuracy overt (simultaneous), accuracy covert (all delays) conditions with maximum scores of 20, 5 and 15 respectively. All scores were stratified by age, but the accuracy (total) and accuracy covert (all delay) conditions are shown for higher level of parental education (LPE).

\section{Normative procedure for all DMS outcome measures}

Norms for DMS outcome measures (accuracy, response latency and probability of an error after an incorrect response outcome measures; see appendix) are established by applying the four-step process described above. Next are an example of this process and accompanying interpretation. For example, suppose that a 6 years old child, whose parents have a low LPE, scored 10 points on DMS accuracy. As detailed above, the regression model for the DMS accuracy score is a power transformation of the test score to achieve better agreement with the distributional assumptions of regression models. Here, the transformed DMS accuracy score equals 4 (Lambda 0.5 applied to 10; Osborne, 2010). The first step of the normative procedure is to calculate the expected score for this child with the regression model presented in Table 4 . This is constant $+0.01 *$ (age child in months average age sample $)+0.42 *$ LPE child $(=4.93+[0.01 *(72-115.59)]+(0.42 * 0)=$ 4.49). The residual is calculated, which is $-0.49(=4-4.49)$. In the third step the residual is standardized $-0.65(-0.49 / 0.75)$. The standardized residual is converted into a percentile value based on the standard normal cumulative distribution. A standardized residual of - 


\section{Chapter 2}

0.65 corresponds with a percentile value of .44 . This means that $44 \%$ of the population of 6 years old children, whose parents have a low LPE obtain a DMS accuracy score that is 10 or lower. The DMS total accuracy test score of this child is therefore within normal limits.

The DMS probability of an error after an incorrect response outcome measure did not meet the normality assumption as evaluated using the Kolmogorov-Smirnov test. Norms for this outcome were therefore, based on the empirical distribution of the standardized residuals (Zhou, 1998). Lastly, this outcome measure showed an effect for Age- $\mathrm{C}^{2}$, which indicates a curved development with age, as can be seen in Figure 6.

The observed mean of 4.5 showed the expected ceiling effect for accuracy in the overt condition (max. 5). Norms for this outcome measure therefore have limited value and in line with observed scores, is the prediction that most children score 3 , 4 , or 5 correct. Therefore, a table with approximate cumulative percentages for the overt scores stratified by age is in the appendix.

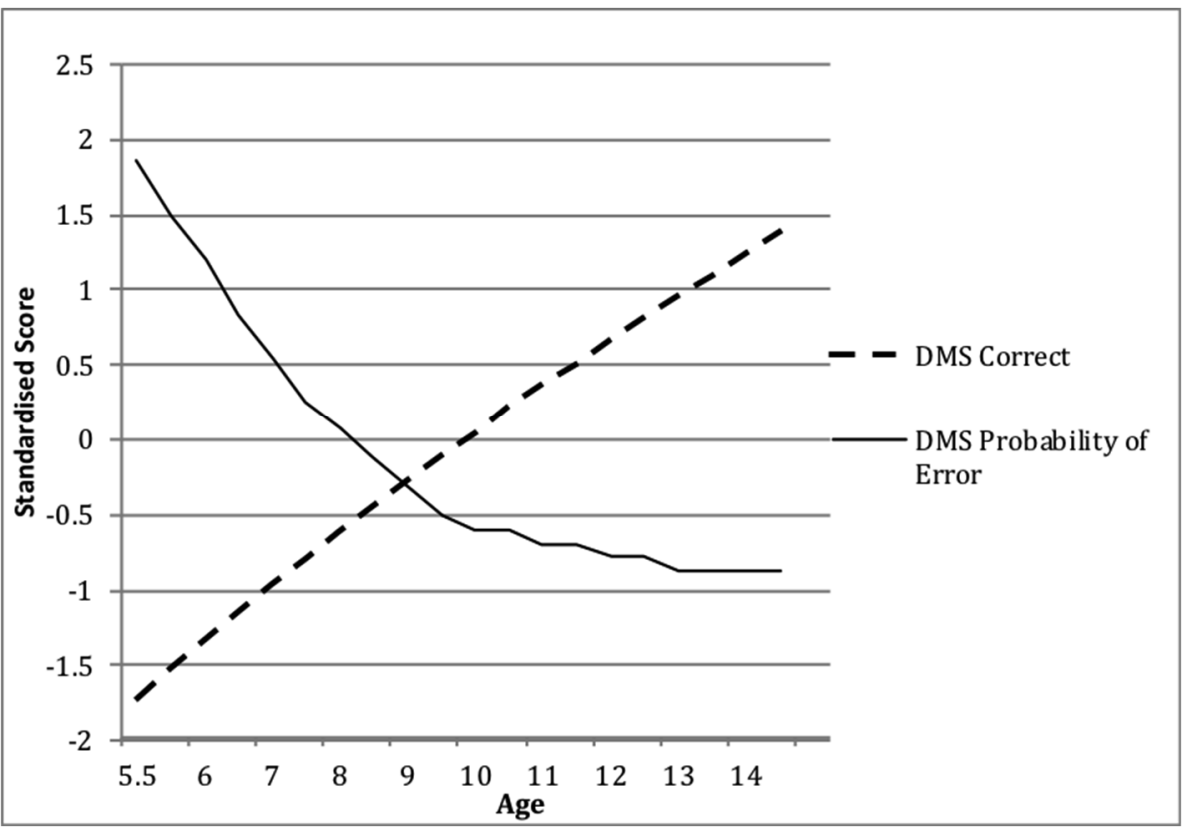

Figure 6. Expected standardized scores based on the regression models for the DMS Accuracy (total) and DMS Probability of error of an incorrect response.

\section{Discussion}

The main aim of the present study was to establish the normal range of performance on three outcome measures for the Delayed Matching to Sample (DMS) test for schoolchildren in the Ukraine. A more novel method called continuous norming was 
used, which allowed for the inclusion of the demographic variables age, sex and LPE simultaneously (Van Breukelen \& Vlaeyen, 2005; Van der Elst et al., 2011). The normal range of performance on the DMS accuracy, latency and probability of an error after an incorrect response outcome measures was reported based on the final regressions (Table 4, Figures $5 \& 6$ ) and expected scores were presented in the appendix. The results of the study are discussed next.

First, the final regression models in this method of continuous norming showed that age influenced DMS accuracy (all conditions), response latency in the overt (simultaneous) condition, and the probability of an error after an incorrect response (see Figure 6). In principle age was linearly related to DMS outcome measures, i.e., children appeared to become more accurate or faster with increasing age. Older children made fewer mistakes, appeared less likely to make an error after an incorrect response, and responded faster than younger children in the overt condition. However, on two DMS outcome measures (i.e., accuracy in the covert condition and probability of an error after an incorrect response) we found a curvilinear age effect. This indicates that development was faster or slower at certain ages compared to other ages. However, specific age groups were not tested on significance of these differences and are not discussed. The observed effects appear nevertheless comparable studies on cross-sectional age-related individual differences in performances of simultaneous visual matching ability, inhibition of responses (i.e., distractors in shape and color) and short-term visual recognition memory of nonverbalisable patterns that are all thought to increase with age (Alloway, Gathercole, Willis, \& Adams, 2004; Davidson et al., 2006; Gathercole, Pickering, Ambridge, \& Wearing, 2004; Korkman, Kemp, \& Kirk, 2001; Luciana \& Nelson, 1998, 2002).

The second finding of the current study on the effects of demographic variables on DMS test performance varied for sex. For one, sex differences were found for DMS response latency in all conditions. Boys responded faster without making more (or fewer) mistakes than girls. This is in line with others who did not find sex differences on the DMS accuracy (Luciana \& Nelson, 1998; Green et al., 2019). Yet, we reported not only on accuracy but on response latency as well, which explains the differences in findings, i.e., an effect of sex on response latency. However, sex explained only $9 \%, 6 \%$ and $5 \%$ of the variance in the DMS response latency for total accuracy, the overt (simultaneous) and covert (all delays) conditions respectively. This is a weak effect. Other explorations for the sex difference in children in this DMS task may need to involve personality characteristics (temperament) or cultural sex differences to explain these findings. It would be conceivable that girls would for example have a higher tendency to (double) check their responses before actually responding in line with studies on sex differences in personality traits (Cloninger, Svrakic, \& Przybeck, 1993; De Bolle et al., 2015).

Third, children's scores of parents with a higher level of education (LPE) were associated with more accuracy on the DMS than those with lower LPE scores. LPE contributed to predicting $27 \%$ and $30 \%$ of the variance in DMS accuracy, and the accuracy covert (delay) condition respectively. The DMS accuracy overt condition did not show an LPE effect. This may support theories that the overt (simultaneous) condition relies on 


\section{Chapter 2}

different processes compared to the covert (delayed) i.e., simultaneous visual matching versus visual short-term recognition memory (Korkman et al., 2001; Mammarella et al., 2008). Equally, no LPE-related differences were found for all DMS response latencies. This seems to be line with Woods, Wyma, Yund, Herron, and Reed (2015) who found in their research in a sample of adults that response latency was not affected by the level of education of participants themselves. This supports our contention that when studying interindividual differences in cognitive functions, and more specifically in cognitive functions needed for DMS performance in individuals (children and adults) both response latency and accuracy need to be assessed in the context of collecting norms. As opposed to traditional norming, the proposed norms for this sample are not all, and not only based on one demographic variable such as age but on the demographic predictors that were significant for the specific DMS outcome measure (i.e., for the DMS accuracy (total) and accuracy covert scores, probability of an error after an incorrect response, and the DMS response latency overt condition, on more than one variable).

In this study, we aimed to reveal the normal range for accuracy and response latency for healthy children taking demographic variables into account. Delineating these different components in a complex cognitive task i.e., accuracy versus response latency, and their respective significant predictors may inform approaches to information processing required for learning in classrooms. The use of both outcome measures could be incorporated in feedback. There is for example increasing attention for formative feedback in classrooms. Instead of a single focus on accuracy (usually summative feedback in the form of amount correct for an end product), teachers are encouraged to formulate and deliver feedback that invites learners to engage (Havnes, Smith, Dysthe, \& Ludvigsen, 2012). Being able to highlight more than one aspect both in learning processes and end results may motivate students. Devising more refined norms may provide both teachers and students with constructive developmental information.

Finally, we posed that continuous norming provides more accurate estimates of children's expected scores and therefore included demographic variables (Mitrushina et al., 2005). The secondary analyses indicated non-significant differences between the age group means for the Ukrainian schoolchildren and the CANTAB ${ }^{\circledR}$ standardized norm on all three DMS outcome measures (also shown in Figures 2-4). This may imply little cultural differences on these DMS outcome measures. However, this comparison was based on the total of the means and standard deviations of age sub groups in the Western norm sample and thus subject to the limitations discussed above. Regression analyses as carried out in this study (i.e., applying the demographic variables age, sex and LPE applied to each data point in the Western norm sample as well) would provide more credence to a comparison between both samples, because it would be a more detailed and accurate analysis (Bechger et al., 2009). Such a comparison might more accurately evaluate whether cultural influences on these outcome measures are (non-)significant.

There are some limitations to this study. The sample consists of primarily rural schools, which may reduce generalizability of these findings. One third of the Ukrainian population is rural (The World Bank, 2016). The economic infrastructure in the Ukraine has 
been limitedly modernized since independence in 1991 and national and regional capitals may receive more funds compared to rural areas (Mokrushyna, 2015). This may support the notion that rural schools in different geographical locations might be in similar (socioeconomic) circumstances that warrant generalizability. Caution might be warranted to application of these findings to urban areas. Children of a similar lower SES in urban areas may for instance have more chances to access computers through better-equipped public facilities, which in turn may lead to more familiarity with computerized tasks resulting in differences in outcomes (Ardila, 1995; Mokrushyna, 2015). Fazeli, Ross, Vance, and Ball (2013) however, did not find differences in cognitive test performance between experienced and non-experienced computer users in an aging study. The latter is supported in the study results here in the absence of significant differences between the age group means of this sample and the CANTAB ${ }^{\circledR}$ standardized norms.

Another limitation concerned sample characteristics. The lower level LPE group was significantly smaller than the high level LPE group. In this method of multiple regression analyses, each data point is applied to the whole sample (Van Breukelen \& Vlaeyen, 2005). The earlier presented argument about the use of continuous and dichotomous variables in these regressions simultaneously applied to the whole sample also concerns the size of the low LPE group and/or a slightly smaller age group of e.g., older children: fewer data points are needed to arrive at statistically valid results (Van Breukelen $\&$ Vlaeyen, 2005). The advantages of regression-based normative methods comes at the cost that thorough checking of the assumptions of these complex models is required. Assumptions of homoscedasticity and normality of the standardized residuals have been thoroughly checked when making these regression-based norms. The standardized residuals of the probability of an error scores were nonGaussian, and this was accounted for by using the empirical distribution of the standardized residuals.

\section{Final conclusions}

This study aimed to establish a normative range for multiple outcome measures of the DMS visual spatial memory task. We found support to derive norms based on age, sex and/or LPE, depending on the DMS outcome measure included. Especially (but not solely) DMS accuracy showed the influence of more than one demographic variable per outcome measure in contrast to more traditionally established norms based on one demographic variable (Cambridge Cognition, 2012). New norms for children in rural Ukrainian schools are proposed in the appendix. 


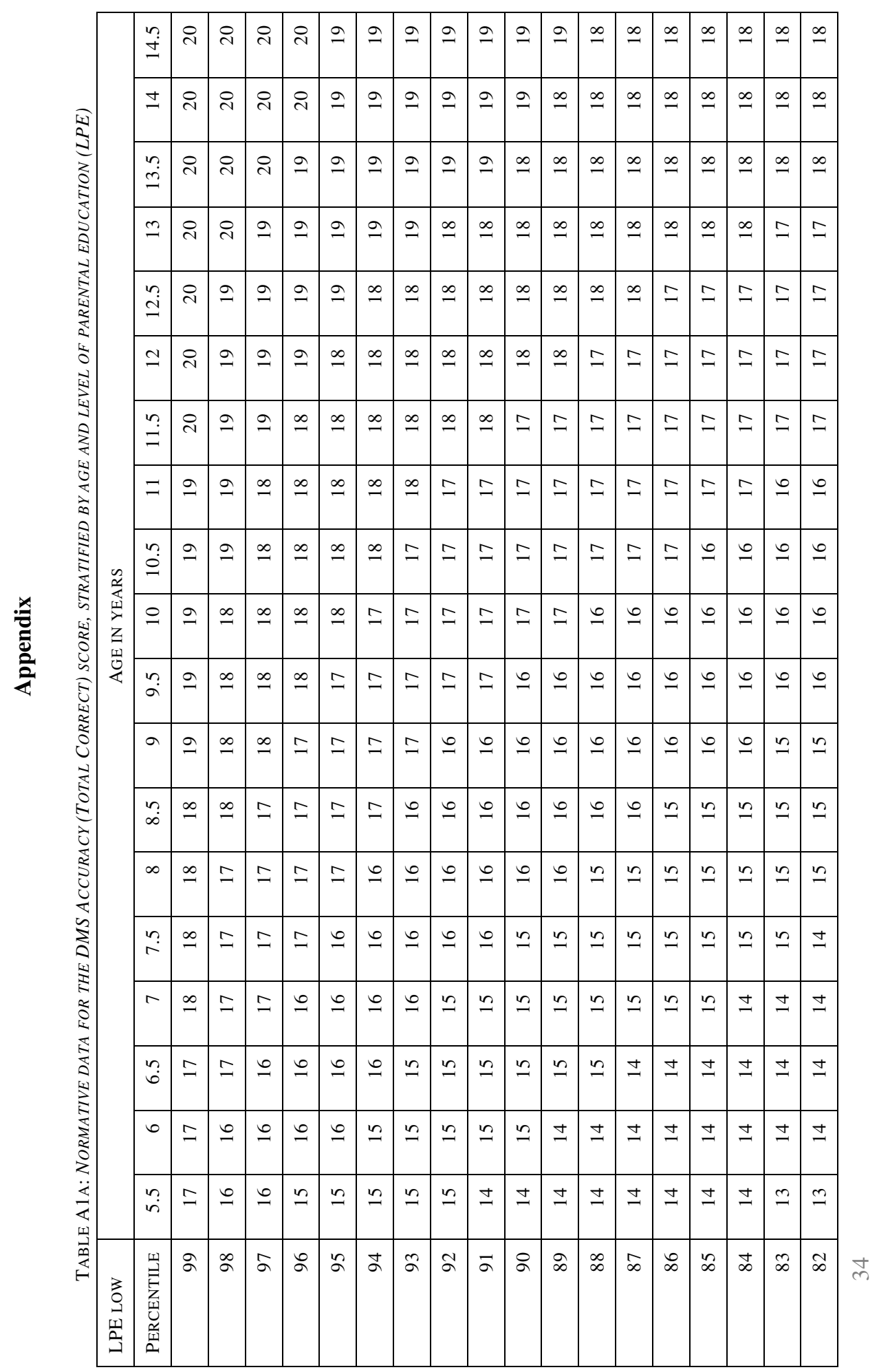




\begin{tabular}{|c|c|c|c|c|c|c|c|c|c|c|c|c|c|c|c|c|c|c|c|c|c|c|}
\hline$\stackrel{\infty}{.}$ & $\stackrel{\infty}{\sim}$ & $\beth$ & $\stackrel{0}{-}$ & \pm & \pm & $\stackrel{m}{2}$ & 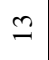 & 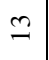 & 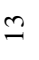 & 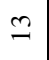 & 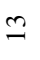 & $\cong$ & 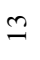 & $\simeq$ & $\simeq$ & $\simeq$ & $\simeq$ & $\simeq$ & $=$ & $=$ & $=$ & 잉 \\
\hline$\stackrel{\infty}{\sim}$ & $\stackrel{\infty}{\infty}$ & $\beth$ & $\stackrel{0}{-}$ & \pm & \pm & $\stackrel{m}{2}$ & 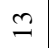 & 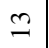 & $\stackrel{m}{-1}$ & $\underline{-}$ & 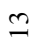 & 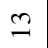 & 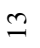 & $\simeq$ & $\simeq$ & $\simeq$ & $\simeq$ & $\simeq$ & $=$ & $=$ & $=$ & $\circ$ \\
\hline$\stackrel{\infty}{-1}$ & $\Xi$ & $\Xi$ & 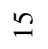 & \pm & $\stackrel{m}{-}$ & $\stackrel{m}{2}$ & 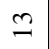 & $\stackrel{m}{2}$ & $\stackrel{m}{2}$ & 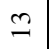 & 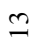 & $\simeq$ & $\simeq$ & $\simeq$ & $\simeq$ & $\simeq$ & $\simeq$ & $=$ & $=$ & $=$ & & $\circ$ \\
\hline$\beth$ & $\simeq$ & 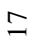 & 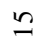 & 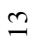 & $\stackrel{m}{-}$ & $\underline{2}$ & $\underline{n}$ & 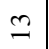 & $\simeq$ & $\simeq$ & $\simeq$ & $\simeq$ & $\simeq$ & $I$ & $\simeq$ & $=$ & $=$ & $=$ & $=$ & $\varrho$ & ㅇ & $a$ \\
\hline 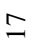 & 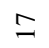 & $\simeq$ & $\cong$ & 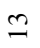 & $\underline{n}$ & $\underline{2}$ & $I$ & $\simeq$ & $\simeq$ & $\simeq$ & $\simeq$ & $\simeq$ & $\simeq$ & $=$ & $=$ & $=$ & $=$ & $=$ & 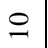 & 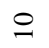 & $a$ & $a$ \\
\hline$\beth$ & 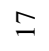 & 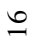 & 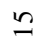 & 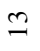 & $\simeq$ & $\simeq$ & $\simeq$ & $\simeq$ & $\simeq$ & $\simeq$ & $\simeq$ & $=$ & $=$ & $=$ & $=$ & $\exists$ & 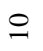 & 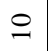 & 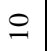 & $a$ & $a$ & $\infty$ \\
\hline$\beth$ & $\stackrel{0}{0}$ & $\stackrel{0}{0}$ & \pm & $\simeq$ & $\simeq$ & $\simeq$ & $\simeq$ & $\simeq$ & $\Xi$ & $=$ & $=$ & $=$ & $=$ & $=$ & 으 & 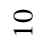 & 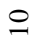 & 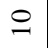 & $a$ & $a$ & $a$ & $\infty$ \\
\hline$\stackrel{0}{0}$ & $\stackrel{0}{ }$ & $\stackrel{\bullet}{0}$ & \pm & $\simeq$ & $\simeq$ & $=$ & $=$ & $=$ & $=$ & $=$ & $=$ & $=$ & 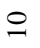 & 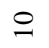 & $\circ$ & 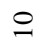 & 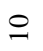 & $a$ & $a$ & $a$ & $\infty$ & $r$ \\
\hline$\stackrel{0}{0}$ & $\stackrel{0}{-}$ & $\stackrel{0}{0}$ & \pm & $\simeq$ & $=$ & $=$ & $=$ & $=$ & $=$ & $=$ & 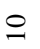 & 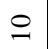 & 인 & 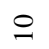 & 으 & $a$ & $a$ & $a$ & $\infty$ & $\infty$ & $\infty$ & $r$ \\
\hline$\stackrel{0}{0}$ & $\stackrel{0}{-}$ & $\because$ & $\stackrel{m}{n}$ & $=$ & $=$ & $=$ & $=$ & 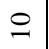 & 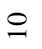 & 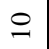 & $\therefore$ & 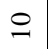 & 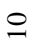 & $a$ & $a$ & $a$ & $a$ & $\infty$ & $\infty$ & $\infty$ & $r$ & 0 \\
\hline$\stackrel{0}{0}$ & $\cong$ & $\cong$ & $\underline{n}$ & $=$ & 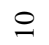 & 은 & 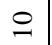 & 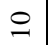 & 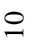 & 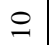 & 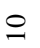 & $a$ & $a$ & $a$ & $a$ & $\infty$ & $\infty$ & $\infty$ & $r$ & $r$ & 0 & in \\
\hline$\cong$ & 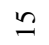 & $\cong$ & $\stackrel{m}{n}$ & $=$ & 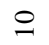 & $\stackrel{0}{ }$ & $\cong$ & 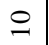 & $a$ & $a$ & $a$ & $a$ & $a$ & $\infty$ & $\infty$ & $\infty$ & $\infty$ & $r$ & $r$ & 0 & 0 & in \\
\hline$\because$ & $\because$ & \pm & $\stackrel{m}{\sim}$ & 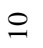 & 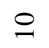 & $a$ & $a$ & $a$ & $a$ & $a$ & $a$ & $\infty$ & $\infty$ & $\infty$ & $\infty$ & $r$ & $r$ & $r$ & 0 & 0 & $n$ & t \\
\hline$\simeq$ & $\cong$ & \pm & $\simeq$ & 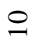 & $a$ & $a$ & $a$ & $a$ & $a$ & $\infty$ & $\infty$ & $\infty$ & $\infty$ & $r$ & r & $r$ & 0 & 0 & in & $n$ & $\nabla$ & $N$ \\
\hline \pm & \pm & $\Xi$ & $\simeq$ & $a$ & $a$ & $a$ & $\infty$ & $\infty$ & $\infty$ & $\infty$ & $\infty$ & $r$ & r & $r$ & r & 0 & 0 & $n$ & $n$ & $\nabla$ & $m$ & 0 \\
\hline \pm & \pm & $\Xi$ & $=$ & $a$ & $\infty$ & $\infty$ & $\infty$ & $\infty$ & $r$ & $r$ & $r$ & $r$ & 0 & 0 & 0 & $n$ & $n$ & $\nabla$ & $\nabla$ & $n$ & 0 & 0 \\
\hline \pm & \pm & 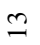 & $=$ & $\infty$ & $\infty$ & $\infty$ & $r$ & $r$ & $r$ & $r$ & 6 & 0 & 6 & $n$ & in & $n$ & $\nabla$ & $m$ & $\sim$ & 0 & 0 & 0 \\
\hline$\cong$ & 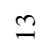 & 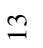 & $=$ & $\infty$ & $r$ & $r$ & $r$ & $r$ & 0 & 0 & 6 & in & in & $n$ & $\nabla$ & $\nabla$ & $m$ & $\sim$ & 0 & 0 & 0 & 0 \\
\hline$\underline{2}$ & 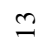 & 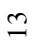 & $\stackrel{-}{0}$ & $r$ & $r$ & 0 & 0 & 0 & 0 & in & $n$ & $n$ & t & $\nabla$ & $m$ & $N$ & 0 & 0 & 0 & 0 & 0 & 0 \\
\hline $\bar{\infty}$ & $\infty$ & $\stackrel{n}{\sim}$ & in & $\sqrt{2}$ & సి & $\stackrel{2}{2}$ & $\stackrel{\infty}{\sim}$ & $\simeq$ & $\stackrel{0}{-}$ & $\underline{\sim}$ & \pm & $\cong$ & $\simeq$ & $=$ & 으 & $a$ & $\infty$ & $r$ & 0 & $n$ & $\nabla$ & $m$ \\
\hline
\end{tabular}




\begin{tabular}{|c|c|c|c|c|c|c|c|c|c|c|c|c|c|c|c|c|c|c|c|c|c|c|}
\hline 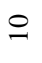 & $\infty$ & & $\stackrel{n}{ \pm}$ & 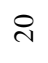 & तิ & $\stackrel{\sim}{ }$ & 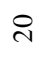 & $\stackrel{\sim}{ }$ & 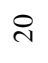 & 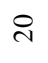 & $\stackrel{\curvearrowright}{~}$ & $\stackrel{i}{ }$ & 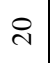 & $\stackrel{i}{ }$ & ते & $\stackrel{\sim}{ }$ & $\stackrel{\sim}{ }$ & $\stackrel{\sim}{ }$ & 2 & 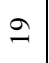 & 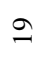 & 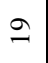 \\
\hline 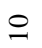 & $\infty$ & & $\Xi$ & $\stackrel{\text { ¿ }}{ }$ & $\stackrel{\sim}{\text { N }}$ & $\stackrel{\sim}{\text { N }}$ & 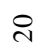 & 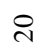 & 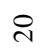 & ㅇ & $\stackrel{\sim}{\circ}$ & ㅇ & $\stackrel{\sim}{\sim}$ & ণ & $\stackrel{\sim}{\sim}$ & $\stackrel{\sim}{ }$ & 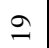 & $\cong$ & 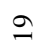 & $\curvearrowright$ & $\stackrel{a}{ }$ & $\cong$ \\
\hline$a$ & $\infty$ & & $\begin{array}{l}n \\
n \\
n\end{array}$ & ఠి & $\stackrel{\curvearrowright}{ }$ & $\stackrel{\sim}{ }$ & $\stackrel{\sim}{ }$ & $\stackrel{\sim}{ }$ & 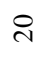 & ఠి & $\stackrel{\sim}{ }$ & $\stackrel{\sim}{ }$ & 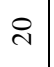 & $\stackrel{i}{ }$ & $\curvearrowright$ & 2 & 2 & 2 & 2 & $\curvearrowright$ & 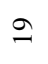 & 2 \\
\hline$a$ & $r$ & & $\stackrel{2}{2}$ & 尺ి & $\stackrel{\sim}{~}$ & ㄱ. & ㄱ. & ㄱ. & ㄱ. & శి & $\stackrel{\sim}{ }$ & ి & $\curvearrowright$ & $\cong$ & 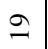 & 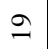 & $\cong$ & $\curvearrowright$ & $\cong$ & $\curvearrowright$ & 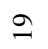 & 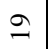 \\
\hline$\infty$ & $r$ & & $\begin{array}{l}n \\
i \\
\end{array}$ & ి & $\stackrel{\sim}{ }$ & ㄱ. & 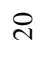 & 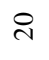 & 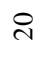 & ㄱ & 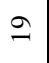 & $\stackrel{2}{ }$ & $\curvearrowright$ & $\stackrel{2}{2}$ & 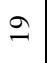 & 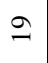 & $\supseteq$ & $\curvearrowright$ & 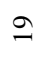 & $\curvearrowright$ & $\stackrel{\infty}{\sim}$ & $\stackrel{\infty}{\sim}$ \\
\hline$\infty$ & 0 & & $\simeq$ & ㄱ. & ㄱ. & $\stackrel{\sim}{\sim}$ & ㄱ. & ㄱ. & ㄱ. & $\stackrel{2}{ }$ & $\curvearrowright$ & $\stackrel{2}{2}$ & 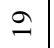 & $\stackrel{2}{2}$ & $\curvearrowright$ & 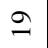 & $\curvearrowright$ & $\stackrel{\infty}{\sim}$ & $\stackrel{\infty}{\sim}$ & $\stackrel{\infty}{\sim}$ & $\stackrel{\infty}{\sim}$ & $\stackrel{\infty}{\sim}$ \\
\hline$r$ & in & & $\stackrel{n}{=}$ & ㄱ. & ㄱ. & $\stackrel{\sim}{\sim}$ & 요 & $\curvearrowright$ & $\curvearrowright$ & $\stackrel{2}{ }$ & 2 & $\stackrel{2}{2}$ & $\curvearrowright$ & $\cong$ & 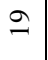 & $\stackrel{\infty}{\sim}$ & $\stackrel{\infty}{-}$ & $\stackrel{\infty}{-}$ & $\stackrel{\infty}{\sim}$ & $\stackrel{\infty}{\sim}$ & $\stackrel{\infty}{-}$ & $\stackrel{\infty}{\sim}$ \\
\hline 0 & $n$ & & $=$ & ㄱ. & ㄱ & $\stackrel{\sim}{\sim}$ & $\stackrel{2}{2}$ & $\curvearrowright$ & $\stackrel{-}{ }$ & $\stackrel{\vartheta}{ }$ & $\curvearrowright$ & $\stackrel{2}{ }$ & $\stackrel{2}{ }$ & $\stackrel{\infty}{-}$ & $\stackrel{\infty}{-}$ & $\stackrel{\infty}{-}$ & $\stackrel{\infty}{-}$ & $\stackrel{\infty}{-}$ & $\stackrel{\infty}{\sim}$ & $\stackrel{\infty}{\sim}$ & $\stackrel{\infty}{\sim}$ & $\stackrel{\infty}{-}$ \\
\hline 0 & $\nabla$ & & ?n & 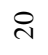 & $\stackrel{\sim}{~}$ & $\stackrel{\sim}{~}$ & 2 & $\curvearrowright$ & 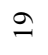 & $\stackrel{\curvearrowright}{2}$ & $\curvearrowright$ & $\stackrel{\infty}{\sim}$ & $\stackrel{\infty}{-}$ & $\stackrel{\infty}{\sim}$ & $\stackrel{\infty}{-}$ & $\stackrel{\infty}{-}$ & $\stackrel{\infty}{-}$ & $\stackrel{\infty}{\longrightarrow}$ & $\stackrel{\infty}{\simeq}$ & $\stackrel{\infty}{\simeq}$ & $\stackrel{\infty}{-}$ & $=$ \\
\hline$n$ & $N$ & $\begin{array}{l}\text { 피 } \\
z\end{array}$ & 0 & ి & $\stackrel{\sim}{ }$ & 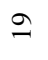 & 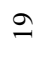 & 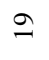 & 9 & $\stackrel{\infty}{-}$ & $\cong$ & $\stackrel{\infty}{\sim}$ & $\stackrel{\infty}{\sim}$ & $\stackrel{\infty}{\sim}$ & $\stackrel{\infty}{\sim}$ & $\stackrel{\infty}{\sim}$ & $\stackrel{\infty}{\sim}$ & $\stackrel{\infty}{\sim}$ & 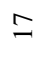 & $\simeq$ & $\beth$ & $\simeq$ \\
\hline$\nabla$ & 0 & 这 & 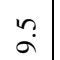 & 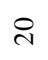 & $\stackrel{\vartheta}{ }$ & $\stackrel{2}{2}$ & $\stackrel{2}{ }$ & $\curvearrowright$ & $\infty$ & $\infty$ & $\stackrel{\infty}{-}$ & $\stackrel{\infty}{\sim}$ & $\stackrel{\infty}{\sim}$ & $\stackrel{\infty}{-}$ & $\stackrel{\infty}{\sim}$ & $=$ & $=$ & $=$ & I & $=$ & I & $=$ \\
\hline$m$ & 0 & & $a$ & 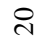 & 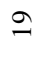 & $\stackrel{2}{2}$ & $\stackrel{2}{2}$ & $\stackrel{\infty}{-}$ & $\stackrel{\infty}{\longrightarrow}$ & $\stackrel{\infty}{-}$ & $\stackrel{\infty}{-}$ & $\stackrel{\infty}{-}$ & $\stackrel{\infty}{\simeq}$ & $=$ & $=$ & $=$ & $=$ & $=$ & 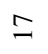 & $\Xi$ & 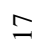 & 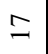 \\
\hline 0 & 0 & & $n$ & $\stackrel{\lambda}{ }$ & $\stackrel{2}{2}$ & 2 & $\stackrel{\infty}{\sim}$ & $\stackrel{\infty}{\sim}$ & $\stackrel{\infty}{-}$ & $\stackrel{\infty}{\sim}$ & $\stackrel{\infty}{-}$ & $\beth$ & $=$ & $=$ & $\approx$ & $\beth$ & $=$ & $\beth$ & $\beth$ & $=$ & $\beth$ & 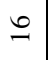 \\
\hline 0 & 0 & & $\infty$ & $\stackrel{2}{ }$ & $\curvearrowright$ & $\stackrel{\infty}{\sim}$ & $\stackrel{\infty}{\sim}$ & $\stackrel{\infty}{\sim}$ & $\stackrel{\infty}{\sim}$ & $\stackrel{\infty}{\sim}$ & 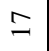 & 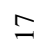 & $=$ & $\simeq$ & $=$ & 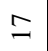 & 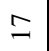 & 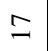 & $\stackrel{\circ}{ }$ & $\stackrel{\circ}{\sim}$ & $\stackrel{\circ}{-}$ & $\stackrel{\bullet}{-}$ \\
\hline 0 & 0 & & $\stackrel{n}{r}$ & $\stackrel{2}{ }$ & 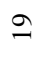 & $\stackrel{\infty}{\sim}$ & $\stackrel{\infty}{\sim}$ & $\stackrel{\infty}{\sim}$ & $=$ & 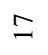 & 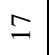 & 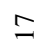 & 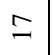 & $\simeq$ & 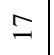 & 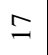 & $\stackrel{\bullet}{-}$ & 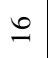 & $\stackrel{\circ}{-}$ & $\stackrel{\circ}{-}$ & $\stackrel{0}{-}$ & $\stackrel{0}{-}$ \\
\hline 0 & 0 & & $r$ & 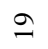 & $\stackrel{\infty}{\sim}$ & $\stackrel{\infty}{\sim}$ & $\stackrel{\infty}{\sim}$ & $\beth$ & 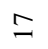 & 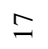 & 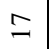 & $I$ & $=$ & $\stackrel{0}{-}$ & $\stackrel{\bullet}{\sim}$ & $\stackrel{0}{=}$ & $\stackrel{\bullet}{-}$ & $\stackrel{0}{ }$ & $\stackrel{0}{ }$ & $\stackrel{0}{-}$ & $\stackrel{0}{-}$ & $\stackrel{0}{ }$ \\
\hline 0 & 0 & & ? & $\curvearrowright$ & $\stackrel{\infty}{\sim}$ & $\stackrel{\infty}{-}$ & $\beth$ & 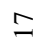 & $I$ & 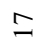 & 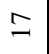 & $I$ & $\varrho$ & $\stackrel{0}{ }$ & $\stackrel{\varrho}{ }$ & $\stackrel{\varrho}{0}$ & 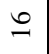 & $\stackrel{\varrho}{0}$ & 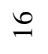 & $\varrho$ & $\cong$ & $\simeq$ \\
\hline 0 & 0 & & 0 & $\stackrel{\infty}{\sim}$ & $\stackrel{\infty}{\sim}$ & $\beth$ & 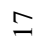 & 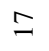 & $\beth$ & $I$ & $\stackrel{\circ}{-}$ & $\stackrel{\circ}{\circ}$ & $\stackrel{0}{-}$ & $\stackrel{0}{\circ}$ & $\stackrel{\bullet}{-}$ & $\stackrel{0}{-}$ & $\stackrel{\circ}{-}$ & $\stackrel{\bullet}{-}$ & $\cong$ & $\simeq$ & $\cong$ & $\simeq$ \\
\hline 0 & 0 & & 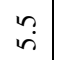 & $\stackrel{\infty}{\sim}$ & $\stackrel{\infty}{\sim}$ & $=$ & $=$ & $\beth$ & $\stackrel{0}{-}$ & $\mathscr{0}$ & $\stackrel{0}{-}$ & $\mathscr{0}$ & $\stackrel{0}{=}$ & $\stackrel{0}{-}$ & $\stackrel{\bullet}{-}$ & $\cong$ & $\cong$ & $\cong$ & $\cong$ & 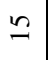 & 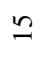 & $\cong$ \\
\hline$\sim$ & - & 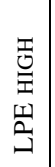 & 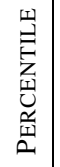 & જे & $\stackrel{\infty}{\sigma}$ & $\hat{a}$ & ஃ & $\approx$ & $\Xi$ & ñ & $\sigma$ & $\bar{a}$ & ஃ & $\infty$ & $\begin{array}{l}\infty \\
\infty\end{array}$ & $\infty$ & $\triangleright$ & $\curvearrowleft$ & $\varpi_{\infty}$ & $\mathscr{\infty}$ & $\underset{\infty}{\infty}$ & $\bar{\infty}$ \\
\hline
\end{tabular}




\begin{tabular}{|c|c|c|c|c|c|c|c|c|c|c|c|c|c|c|c|c|c|c|c|c|c|c|}
\hline$\stackrel{2}{2}$ & $\curvearrowright$ & 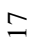 & $\stackrel{\bullet}{\sim}$ & $\cong$ & 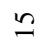 & $\because$ & $\cong$ & $\simeq$ & $\cong$ & 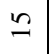 & 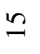 & $\because$ & $\because$ & \pm & \pm & \pm & \pm & \pm & 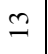 & $\mathscr{n}$ & 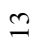 & $\simeq$ \\
\hline 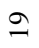 & 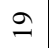 & $\beth$ & $\stackrel{0}{-}$ & $\because$ & $\cong$ & 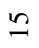 & 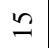 & 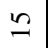 & 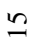 & $\simeq$ & \pm & \pm & \pm & \pm & \pm & \pm & \pm & 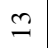 & 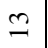 & $\stackrel{m}{\longrightarrow}$ & $\simeq$ & $\simeq$ \\
\hline 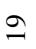 & $\stackrel{\infty}{\sim}$ & 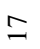 & $\cong$ & $\cong$ & $\because$ & $\cong$ & $\cong$ & $\cong$ & $\Xi$ & \pm & \pm & \pm & \pm & $\Xi$ & $\Xi$ & 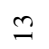 & 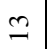 & $\cong$ & 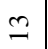 & $\simeq$ & $\simeq$ & $=$ \\
\hline$\cong$ & $\stackrel{\infty}{\sim}$ & $\simeq$ & $\cong$ & $\cong$ & $\cong$ & \pm & \pm & \pm & \pm & \pm & \pm & \pm & \pm & $\underline{n}$ & 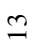 & $\underline{n}$ & $\underline{\sim}$ & $\cong$ & $\simeq$ & $\simeq$ & $\simeq$ & $=$ \\
\hline 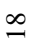 & $\cong$ & $\stackrel{0}{0}$ & $\cong$ & \pm & \pm & \pm & \pm & \pm & \pm & \pm & \pm & $\cong$ & $\cong$ & $\underline{n}$ & 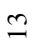 & $\underline{n}$ & $\underline{2}$ & $I$ & $\simeq$ & 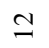 & $=$ & $=$ \\
\hline$\stackrel{\infty}{-}$ & $\infty$ & $\stackrel{0}{0}$ & 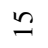 & \pm & \pm & \pm & \pm & \pm & $\Xi$ & 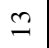 & 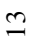 & $\cong$ & $\cong$ & $\stackrel{m}{\sim}$ & $\stackrel{m}{=}$ & $\simeq$ & $\simeq$ & $\simeq$ & $\simeq$ & $\exists$ & $=$ & $\circ$ \\
\hline$\stackrel{\infty}{-}$ & $=$ & $\stackrel{0}{0}$ & \pm & \pm & \pm & \pm & 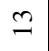 & 2 & 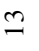 & 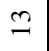 & 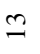 & $\stackrel{m}{-}$ & 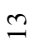 & $\simeq$ & $\simeq$ & $\simeq$ & $\simeq$ & $\simeq$ & $=$ & $=$ & $=$ & 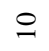 \\
\hline$\stackrel{\infty}{-}$ & $\Xi$ & $\stackrel{0}{0}$ & $\Xi$ & 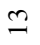 & 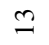 & 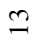 & 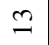 & 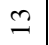 & $\cong$ & 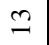 & 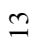 & $\simeq$ & $\simeq$ & $I$ & $\simeq$ & $\simeq$ & $\simeq$ & $=$ & $=$ & $=$ & 으 & $a$ \\
\hline I & $=$ & $\because$ & \pm & 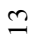 & 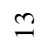 & $\stackrel{m}{2}$ & 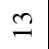 & 2 & $\stackrel{m}{2}$ & $\simeq$ & $\simeq$ & $\simeq$ & $\simeq$ & $\simeq$ & $\simeq$ & $=$ & $=$ & $=$ & $=$ & 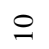 & 인 & $a$ \\
\hline$\beth$ & $=$ & $\because$ & $\stackrel{m}{\sim}$ & $\cong$ & 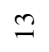 & $\stackrel{2}{2}$ & $I$ & $\simeq$ & $\simeq$ & $\simeq$ & $\simeq$ & $\simeq$ & $\simeq$ & $\exists$ & $=$ & $=$ & $=$ & $\varrho$ & $\varrho$ & 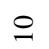 & $a$ & $\infty$ \\
\hline$\beth$ & $\because$ & $\because$ & $\stackrel{m}{\sim}$ & $\simeq$ & $\simeq$ & $\simeq$ & $\simeq$ & $\simeq$ & $\simeq$ & $\simeq$ & $\simeq$ & $=$ & $=$ & $=$ & $=$ & $=$ & 0 & 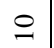 & $\varrho$ & $a$ & $a$ & $\infty$ \\
\hline$\beth$ & 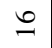 & $\because$ & $\stackrel{m}{-}$ & $\simeq$ & $\simeq$ & $\simeq$ & $\simeq$ & $\simeq$ & $\simeq$ & $=$ & $=$ & $=$ & $=$ & $=$ & 은 & 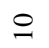 & $\varrho$ & 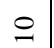 & $a$ & $a$ & $\infty$ & $r$ \\
\hline$\stackrel{0}{0}$ & $\stackrel{0}{ }$ & \pm & 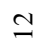 & $\simeq$ & $\simeq$ & $\simeq$ & $=$ & $=$ & $=$ & $=$ & $=$ & $=$ & 은 & 0 & 은 & 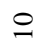 & 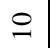 & $a$ & $a$ & $\infty$ & $\infty$ & $r$ \\
\hline$\stackrel{0}{0}$ & 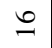 & \pm & $\simeq$ & $=$ & $=$ & $=$ & $=$ & $=$ & $=$ & $=$ & 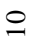 & $\stackrel{0}{ }$ & 으 & 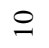 & 으 & $a$ & $a$ & $a$ & $\infty$ & $\infty$ & $r$ & 0 \\
\hline$\underline{0}$ & 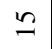 & \pm & $\simeq$ & $=$ & $=$ & $=$ & $=$ & $=$ & & 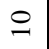 & 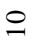 & 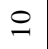 & 은 & $a$ & $a$ & $a$ & $a$ & $\infty$ & $\infty$ & -1 & $r$ & 0 \\
\hline$\stackrel{0}{0}$ & $\cong$ & 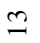 & $=$ & $=$ & $=$ & 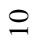 & $\cong$ & 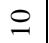 & 으 & 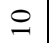 & 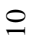 & $a$ & $a$ & $a$ & $a$ & $\infty$ & $\infty$ & $\infty$ & $r$ & $r$ & 0 & in \\
\hline$\because$ & 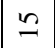 & $\stackrel{m}{2}$ & $=$ & 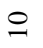 & 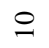 & 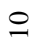 & $\cong$ & 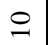 & 이 & $a$ & $a$ & $a$ & $a$ & $a$ & $\infty$ & $\infty$ & $\infty$ & $r$ & $r$ & 0 & $n$ & t \\
\hline$\because$ & 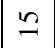 & $\stackrel{m}{2}$ & $=$ & 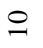 & 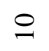 & $\stackrel{0}{ }$ & $a$ & $a$ & $a$ & $a$ & $a$ & $a$ & $\infty$ & $\infty$ & $\infty$ & $r$ & $r$ & $r$ & 0 & $n$ & $\nabla$ & N \\
\hline$\because$ & \pm & $\simeq$ & $\stackrel{-}{0}$ & $a$ & $a$ & $a$ & $a$ & $a$ & $a$ & $\infty$ & $\infty$ & $\infty$ & $\infty$ & $r$ & r & $r$ & 0 & 0 & $n$ & $\nabla$ & $m$ & 0 \\
\hline$\infty$ & $\approx$ & in & $\approx$ & $\stackrel{\sim}{\sim}$ & 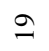 & $\stackrel{\infty}{\sim}$ & 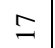 & $\stackrel{\bullet}{0}$ & 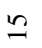 & \pm & $\cong$ & $\simeq$ & $=$ & 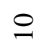 & $a$ & $\infty$ & $r$ & 0 & $n$ & $\nabla$ & $m$ & N \\
\hline
\end{tabular}




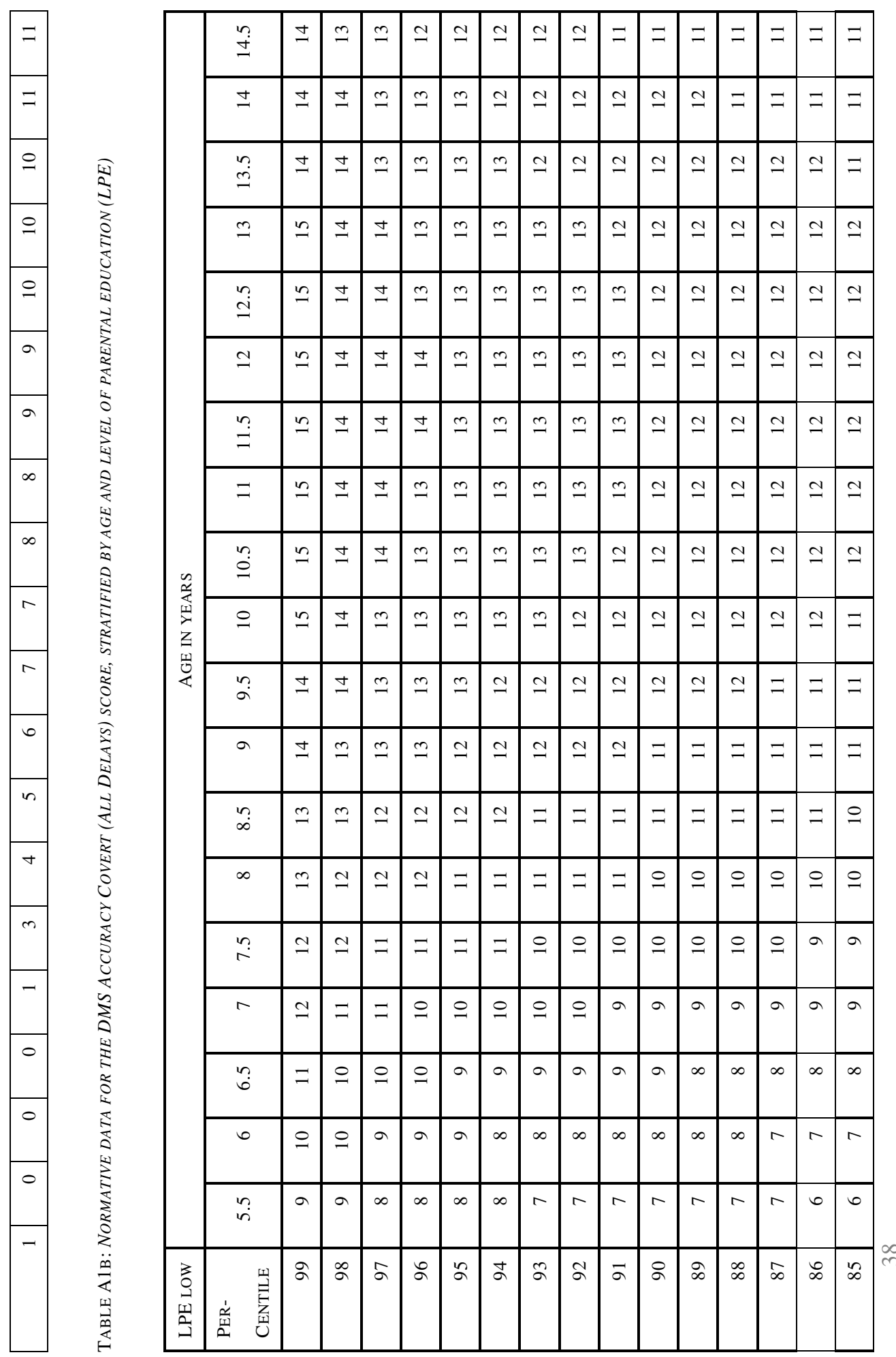




\begin{tabular}{|c|c|c|c|c|c|c|c|c|c|c|c|c|c|c|c|c|c|c|c|c|}
\hline$=$ & $=$ & 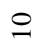 & 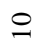 & $\stackrel{0}{-}$ & $\stackrel{ }{-}$ & $\infty$ & N & 0 & 0 & 0 & 0 & 6 & 0 & 0 & 6 & ○ & $n$ & in & $n$ & $n$ \\
\hline$=$ & $=$ & $=$ & $=$ & $=$ & 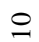 & $a$ & $r$ & $r$ & $r$ & $r$ & 0 & 0 & 0 & 0 & 6 & 0 & 0 & 0 & 0 & $n$ \\
\hline$=$ & $=$ & $=$ & $=$ & $=$ & $=$ & $a$ & $N$ & $r$ & $r$ & $r$ & $r$ & $r$ & $r$ & 0 & 0 & 0 & 0 & 0 & 0 & 0 \\
\hline$\simeq$ & $=$ & $=$ & $=$ & $=$ & $=$ & $a$ & $\infty$ & $r$ & $r$ & $r$ & $r$ & $r$ & $r$ & $r$ & $r$ & 0 & 0 & 0 & 0 & 0 \\
\hline I & $\simeq$ & $\simeq$ & $=$ & $=$ & $=$ & $a$ & $\infty$ & $r$ & $r$ & $r$ & $r$ & $r$ & $r$ & $r$ & $r$ & $r$ & 0 & 0 & 0 & 0 \\
\hline I & 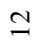 & $\simeq$ & $=$ & $=$ & $=$ & $a$ & $\infty$ & $r$ & $r$ & $r$ & $r$ & $r$ & $r$ & $r$ & $r$ & $r$ & $r$ & 0 & 0 & 6 \\
\hline $\mathrm{N}$ & $\mathrm{I}$ & $\simeq$ & $\simeq$ & $=$ & $=$ & $a$ & $\infty$ & $r$ & $r$ & $r$ & $r$ & $r$ & $r$ & $r$ & $r$ & $r$ & $r$ & 0 & 0 & 0 \\
\hline$\simeq$ & 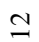 & $\simeq$ & $=$ & $=$ & $=$ & $a$ & $\infty$ & $r$ & $r$ & $r$ & $r$ & $r$ & $r$ & $r$ & $r$ & $r$ & 0 & 0 & 0 & 6 \\
\hline $\mathrm{I}$ & $=$ & $=$ & $=$ & $=$ & $=$ & $a$ & $\infty$ & $r$ & $r$ & $r$ & $r$ & $r$ & $r$ & $r$ & $r$ & 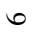 & 0 & 0 & 0 & 0 \\
\hline$=$ & $=$ & $=$ & $=$ & $=$ & $=$ & $a$ & $r$ & $r$ & $r$ & $r$ & $r$ & $r$ & $r$ & 0 & 6 & 0 & 6 & 0 & 6 & 6 \\
\hline$=$ & $=$ & $=$ & $=$ & $=$ & 으 & $a$ & $r$ & $r$ & $r$ & $r$ & 6 & 0 & 0 & 0 & 6 & 0 & 0 & 0 & 0 & $n$ \\
\hline$=$ & $=$ & $=$ & 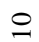 & 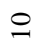 & 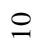 & $\infty$ & $r$ & 0 & 0 & 0 & 6 & 0 & 0 & 0 & 6 & 6 & $n$ & $n$ & $n$ & $n$ \\
\hline 0 & 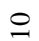 & 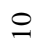 & 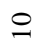 & 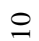 & 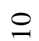 & $\infty$ & 0 & 0 & 0 & 0 & 0 & 0 & 0 & $n$ & $n$ & $n$ & $n$ & $n$ & $n$ & $n$ \\
\hline 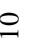 & 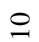 & 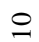 & 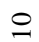 & $a$ & $a$ & $r$ & 0 & $n$ & $n$ & $n$ & $n$ & $n$ & $n$ & in & $n$ & in & $n$ & $\forall$ & $\forall$ & $\forall$ \\
\hline$a$ & $a$ & $a$ & $a$ & $a$ & $\infty$ & $r$ & in & $n$ & $n$ & in & $n$ & $n$ & $\nabla$ & $\nabla$ & $\forall$ & $\forall$ & $\forall$ & $\nabla$ & $\forall$ & $\forall$ \\
\hline$a$ & $a$ & $\infty$ & $\infty$ & $\infty$ & $\infty$ & 6 & in & $\forall$ & $\forall$ & $\forall$ & $\nabla$ & $\forall$ & $\nabla$ & $\forall$ & $\forall$ & $m$ & $m$ & $m$ & $m$ & $m$ \\
\hline$\infty$ & $\infty$ & $\infty$ & $\infty$ & $\infty$ & $r$ & 6 & $\forall$ & $\forall$ & $m$ & $m$ & $m$ & $m$ & $m$ & $m$ & $m$ & $m$ & $m$ & $m$ & $N$ & $N$ \\
\hline$r$ & $r$ & $r$ & $r$ & $r$ & 6 & $n$ & $m$ & $m$ & $m$ & $m$ & $m$ & N & N & $N$ & $N$ & $N$ & N & N & $N$ & - \\
\hline 6 & 6 & 6 & 0 & 6 & $n$ & $\nabla$ & $N$ & N & $N$ & N & 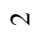 & N & - & - & - & - & - & - & - & - \\
\hline 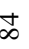 & $\infty$ & $\infty$ & $\infty$ & $\stackrel{\infty}{\infty}$ & $\stackrel{n}{\sim}$ & $\stackrel{\circ}{n}$ & $\stackrel{n}{\sim}$ & 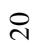 & $\stackrel{2}{=}$ & $\infty$ & I & $\underline{\sigma}$ & $\cong$ & \pm & $m$ & 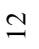 & $=$ & $\stackrel{0}{ }$ & $a$ & $\infty$ \\
\hline
\end{tabular}




\begin{tabular}{|c|c|c|c|c|c|c|}
\hline$n$ & in & $\nabla$ & $\nabla$ & $\nabla$ & $m$ & $n$ \\
\hline$n$ & $n$ & $n$ & $n$ & $\nabla$ & $\nabla$ & $m$ \\
\hline in & in & in & $n$ & $n$ & $\forall$ & $m$ \\
\hline 6 & 6 & $n$ & $n$ & $n$ & $\nabla$ & $\nabla$ \\
\hline 6 & 6 & $n$ & $n$ & $n$ & $\forall$ & $\nabla$ \\
\hline 6 & 0 & 0 & in & $n$ & in & $\nabla$ \\
\hline 0 & 6 & 0 & $n$ & $n$ & $n$ & $\nabla$ \\
\hline 6 & 0 & $n$ & $n$ & $n$ & $n$ & $\nabla$ \\
\hline 6 & 6 & $n$ & $n$ & $n$ & $\forall$ & $\nabla$ \\
\hline 0 & in & $n$ & $n$ & $n$ & $\nabla$ & $\nabla$ \\
\hline$n$ & $n$ & $n$ & $n$ & $\forall$ & $\forall$ & $m$ \\
\hline$n$ & $n$ & $n$ & $\nabla$ & $\nabla$ & $\forall$ & $m$ \\
\hline$\forall$ & $\forall$ & $\forall$ & $\nabla$ & $\nabla$ & $m$ & $N$ \\
\hline$\forall$ & $\nabla$ & $\forall$ & $m$ & $m$ & $m$ & $N$ \\
\hline$m$ & $m$ & $m$ & $m$ & $N$ & $N$ & - \\
\hline$m$ & $m$ & $N$ & $N$ & $N$ & - & - \\
\hline 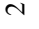 & $N$ & $N$ & - & - & - & 0 \\
\hline- & - & - & - & 0 & 0 & 0 \\
\hline 0 & 0 & 0 & 0 & 0 & 0 & 0 \\
\hline$r$ & 6 & in & $\nabla$ & $m$ & $N$ & - \\
\hline
\end{tabular}

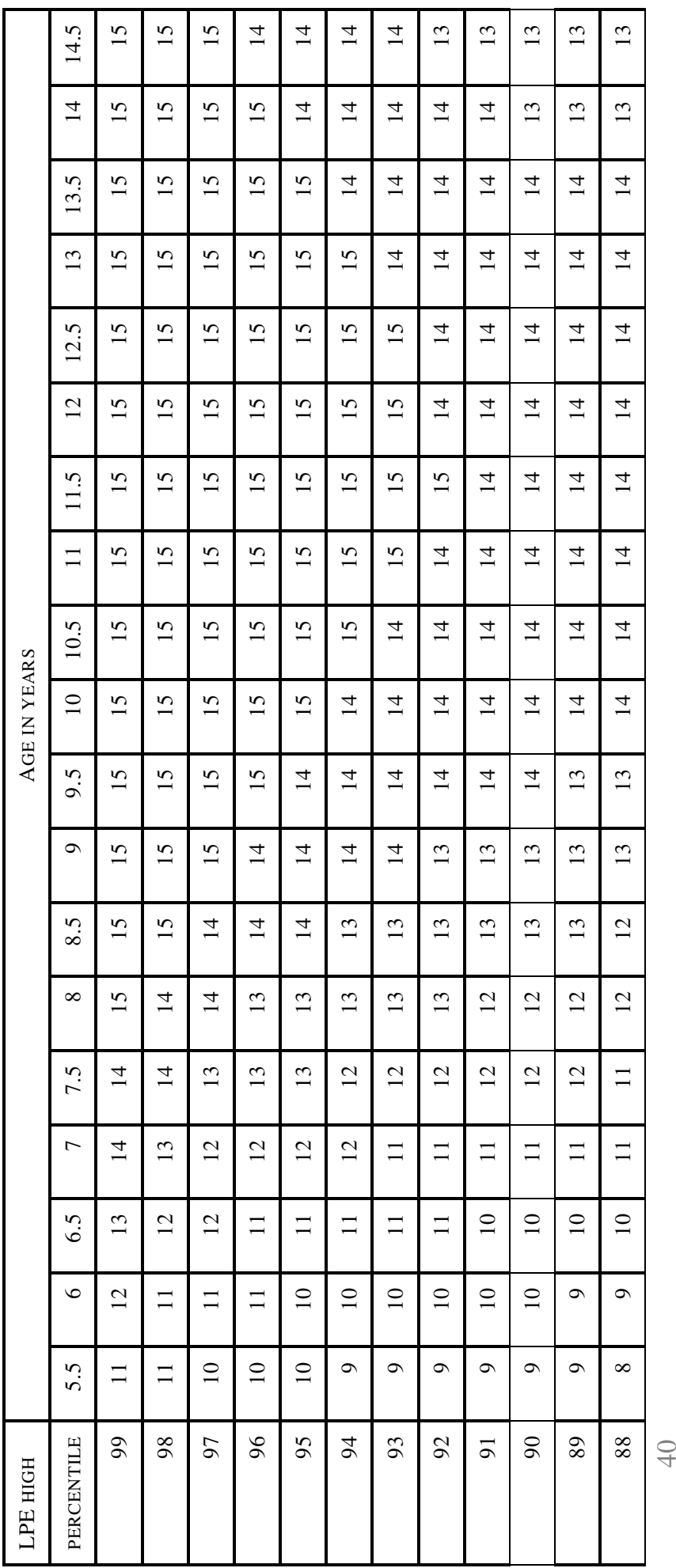




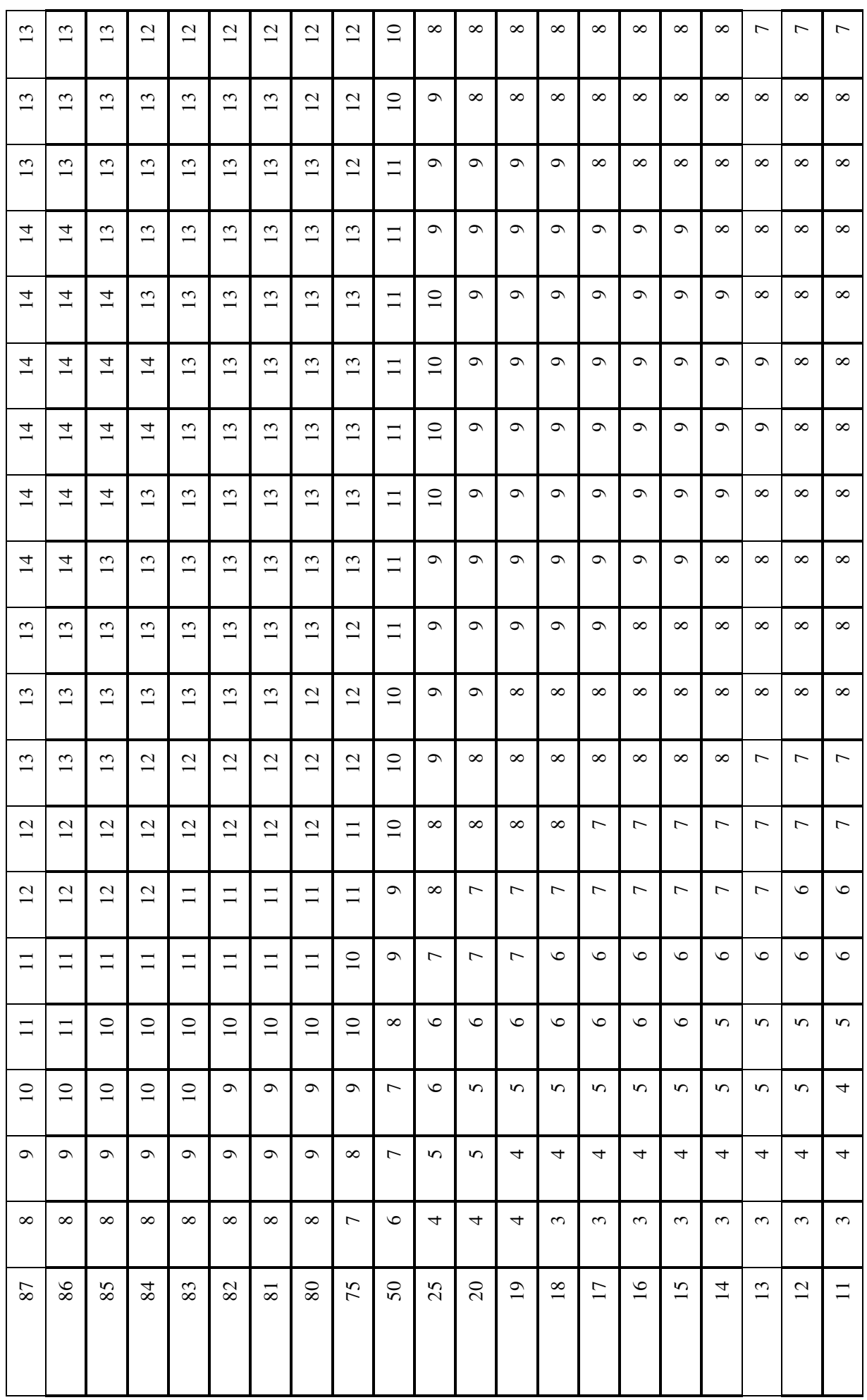



Table A1c: Cumulative percentages for the DMS Accuracy Overt (Simultaneous) score stratified by age from 4 correct and lower (as the rest achieves the maximum score of 5 correct, i.e., 100\%)

\begin{tabular}{|c|c|c|c|}
\hline & \multirow{8}{*}{ 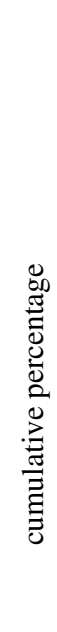 } & \multicolumn{2}{|l|}{ Age } \\
\hline Raw score & & 6 to 9 years old & 10 to 14 years old \\
\hline for 4 correct and lower & & $\%$ & $\%$ \\
\hline 4 correct & & 47 & 25 \\
\hline 3 correct & & 14 & 4 \\
\hline 2 correct & & 8 & 3 \\
\hline 1 correct & & 2 & 1 \\
\hline 0 correct & & 1 & 0 \\
\hline
\end{tabular}




\section{Chapter 2}

Table A2a: Normative data for the DMS Response Latency (accuracy and accuracy covert condition) stratified by sex

\begin{tabular}{|c|c|c|c|c|}
\hline \multirow[b]{2}{*}{$\begin{array}{l}\text { Percentil } \\
\text { e }\end{array}$} & \multicolumn{2}{|l|}{ Male } & \multicolumn{2}{|l|}{ Female } \\
\hline & $\begin{array}{l}\text { RT } \\
\text { accuracy }\end{array}$ & $\begin{array}{l}\text { RT accuracy } \\
\text { covert } \\
\text { (all delays) }\end{array}$ & RT accuracy & $\begin{array}{l}\text { RT accuracy covert } \\
\text { (all delays) }\end{array}$ \\
\hline & & (covert) & & (covert) \\
\hline 99 & 1477.1 & 1929,8 & 2087,9 & 2155,3 \\
\hline 95 & 2162.7 & 2277,6 & 2773.4 & 2630,3 \\
\hline 90 & 2528.1 & 2536,8 & 3138.9 & 2950,2 \\
\hline 85 & 2774.7 & 2738,0 & 3385.5 & 3190,4 \\
\hline 80 & 2970.7 & 2912,4 & 3581.5 & 3395,0 \\
\hline 75 & 3138.8 & 3071,9 & 3749.6 & 3579,8 \\
\hline 50 & 3817.3 & 3805,2 & 4428.1 & 4410,7 \\
\hline 25 & 4495.9 & 4674,3 & 5106.6 & 5371,8 \\
\hline 20 & 4664.0 & 4909,7 & 5274.8 & 5629,3 \\
\hline 15 & 4860.0 & 5193,9 & 5470.7 & 5939,0 \\
\hline 10 & 5106.6 & 5566,3 & 5717.3 & 6342,8 \\
\hline 5 & 5472.0 & 6147,9 & 6082.8 & 6970,3 \\
\hline 1 & 6157.6 & 7332,1 & 6768.4 & 8237,9 \\
\hline
\end{tabular}

* Overt condition stratified by age; average of all ages for percentile

Note: only scores corresponding with percentiles 1, 5, 10, 15, 20, 25, 50, 75, 80, 85, 90, 95 and 99 are given. 


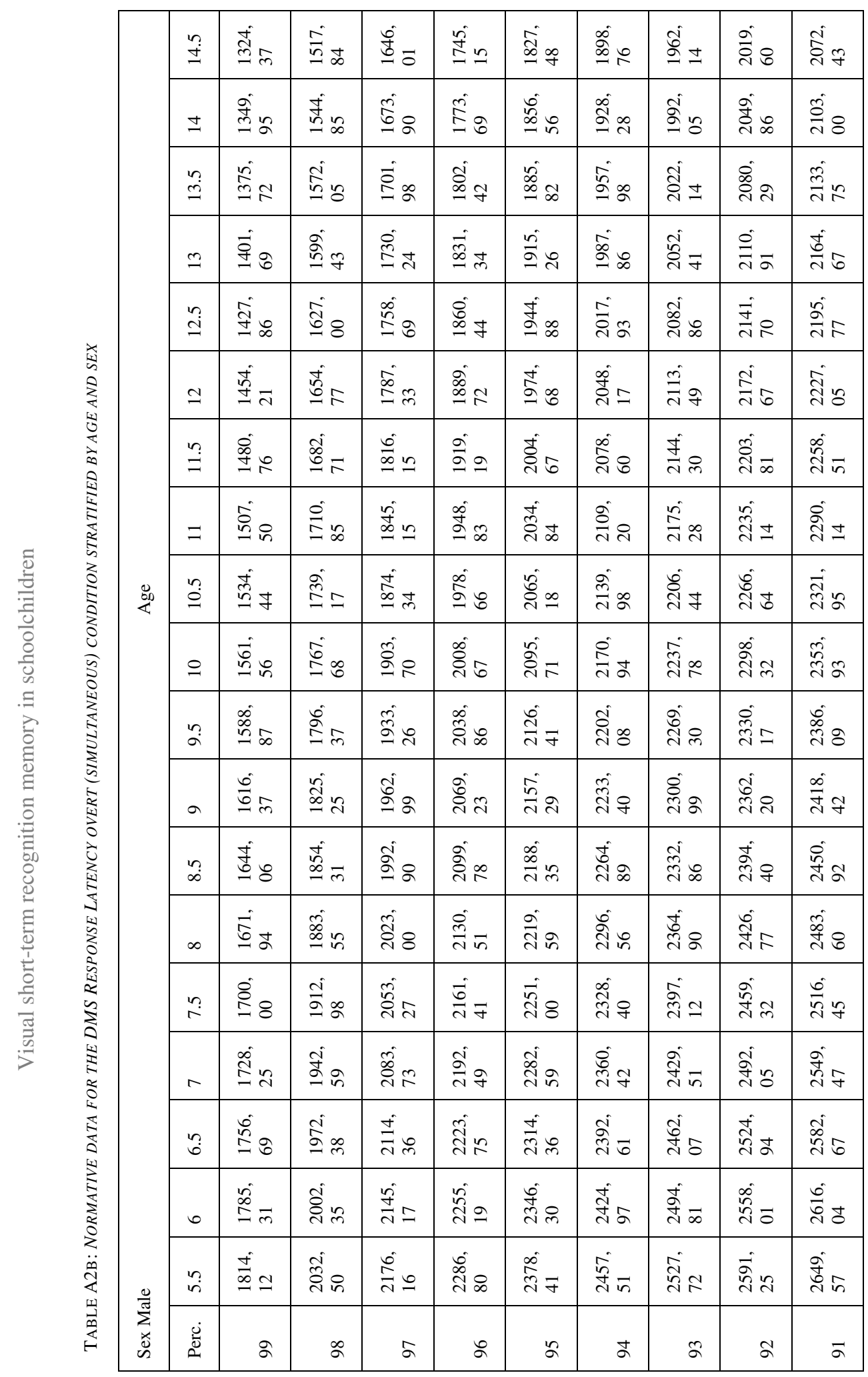




\begin{tabular}{|c|c|c|c|c|c|c|c|c|c|c|}
\hline 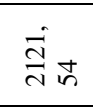 & 응 & సี่으 & ปี่ & ते & लें & 栉= & 亲品 & 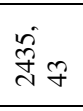 & 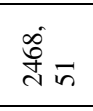 & 离 \\
\hline 窝 & $\begin{array}{l}\infty \\
\stackrel{\sim}{N}\end{array}$ & ปี่ & 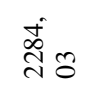 & 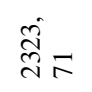 & 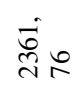 & $\begin{array}{l}\infty \\
\text { वें }\end{array}$ & $\begin{array}{l}\text { Do } \\
\text { d: }\end{array}$ & $\begin{array}{l}\infty \\
\text { do } \\
\text { dै }\end{array}$ & 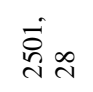 & ते \\
\hline$\frac{\infty}{\sim} \stackrel{\infty}{\sim}$ & तें & 茫 & 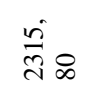 & $\begin{array}{l}\text { vi } \\
\text { di }\end{array}$ & 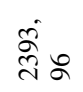 & 栾 & 总 & 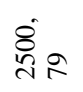 & 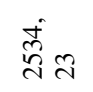 & สิ \\
\hline$\stackrel{\vec{d}}{\vec{d}}+$ & $\overrightarrow{\mathrm{d}}$ & 㝕 & 守 & $\begin{array}{l}\infty \\
\tilde{\nabla} \\
\tilde{\infty}\end{array}$ & 密 & 密 & 焉= & 趂 & 它 & 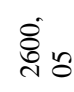 \\
\hline đึํำ & สิ่ & ल) & iิ̀- & 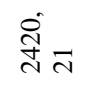 & $\begin{array}{l}\infty \\
\stackrel{\infty}{a} \infty \\
\stackrel{d}{\infty} \infty\end{array}$ & $\stackrel{8}{\stackrel{d}{d}=}$ & तुં & 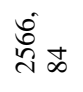 & 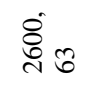 & तi \\
\hline 突 & 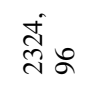 & 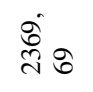 & 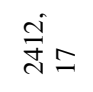 & $\begin{array}{l}\dot{v} \\
\text { yेd } \\
\text { N }\end{array}$ & 高 & तิ่ & 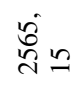 & : & 芯 & 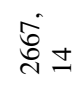 \\
\hline बेंે & 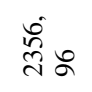 & $\overrightarrow{\vec{J}}$ & $\underset{d}{\text { 寺 }}$ & 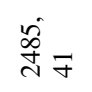 & 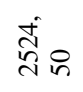 & 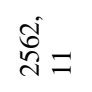 & 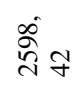 & लु & 递 & ¿্خ \\
\hline $\overrightarrow{\mathrm{f}} \overrightarrow{\mathrm{d}} \mathrm{d}$ & $\begin{array}{l}\dot{0} \\
\dot{\gamma} \cong\end{array}$ & 蔮吕 & 守 & $\frac{\infty}{\vec{d}} \sqrt{d}$ & 究吕 & $\begin{array}{l}\text { वें } \\
\text { तु }\end{array}$ & $\overrightarrow{\vec{d}} \dot{\vec{d}}$ & 递 & $\overrightarrow{\vec{D}_{i}}$ & 䒿。 \\
\hline îं & $\overrightarrow{\overrightarrow{\mathrm{g}}} \underset{\mathrm{d}}{\infty}$ & 号さ & 옹ㅇ & तें & बें & 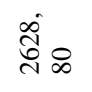 & 客 & 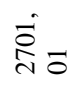 & 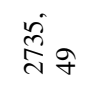 & 옹 \\
\hline 总。 & 志。 & 㞭尺 & 晃 & $\begin{array}{l}\vec{D} \\
\text { o. } \\
\text { an }\end{array}$ & 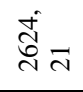 & 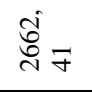 & 商 & 芬 & 过 & ه্心 \\
\hline 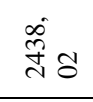 & 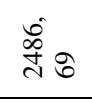 & तี่ & 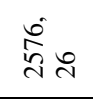 & $\overrightarrow{\tilde{C}}$ & 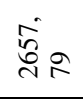 & 递充 & त̂. & 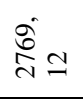 & 舒 & 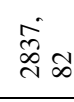 \\
\hline 守 & 공요 & 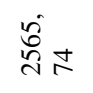 & 产的 & $\overrightarrow{\vec{b}} \dot{d} q$ & $\overrightarrow{\vec{g}}$ & 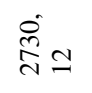 & 密问 & 离 & $\begin{array}{l}\infty \\
\tilde{N}^{0}\end{array}$ & id ? \\
\hline 令 & तें & 今े & 勇 & 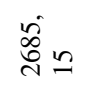 & 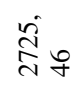 & 晜 & 离过 & 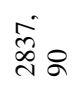 & 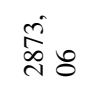 & 寄 \\
\hline $\begin{array}{l}\text { तूँ } \\
\text { तें }\end{array}$ & $\begin{array}{l}\infty \\
\text { तึं }\end{array}$ & 苟守子 & 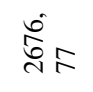 & $\stackrel{\partial}{\vec{\lambda}}+$ & 突㶽 & $\begin{array}{l}\infty \\
\text { ป̀ } \\
\text { ind }\end{array}$ & 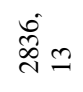 & 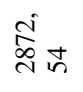 & 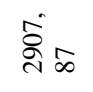 & सैं \\
\hline $\begin{array}{l}\text { वें. } \\
\text { तె }\end{array}$ & हैं & 客 & 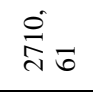 & 令ㅇ & 究 & 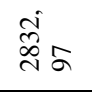 & ס̊: & 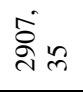 & व̃. & ते ली \\
\hline : & 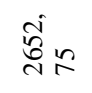 & ब्वें & $\underset{d}{\mathbb{d}} 8$ & $\begin{array}{l}\dot{\infty} \\
\stackrel{\sim}{\sim}\end{array}$ & 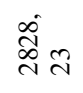 & 通 & 突施 & 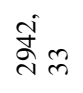 & 㐫8 & ㅎํ요 \\
\hline 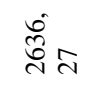 & 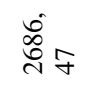 & 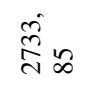 & $\stackrel{\infty}{\stackrel{\infty}{\Delta} \vec{\infty}}$ & 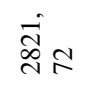 & 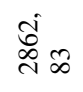 & 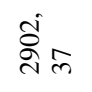 & 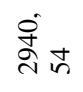 & 客子 & $\overrightarrow{\tilde{D}}$ & 离 \\
\hline 产 & तิ่ & 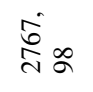 & $\underset{\vec{D}}{\vec{d}}=$ & $\begin{array}{l}\infty \\
\infty \\
\substack{\sim \\
\sim}\end{array}$ & 商问 & 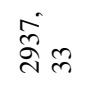 & مُ & 离市 & $\underbrace{\infty}_{\infty} \infty$ & $\stackrel{\infty}{\infty}$ \\
\hline 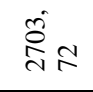 & 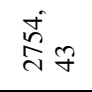 & 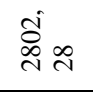 & 豙。 & 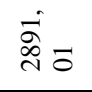 & 莡 & 苓年 & 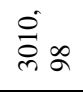 & io & 总 & $\stackrel{\mathrm{m}}{\mathrm{m}}$ \\
\hline 8 & $\stackrel{\infty}{ }$ & $\infty$ & $\bar{\infty}$ & $\infty$ & $\infty$ & $\underset{\infty}{ \pm}$ & $\infty$ & $\infty$ & $\vec{\infty}$ & \\
\hline
\end{tabular}




\begin{tabular}{|c|c|c|c|c|c|c|c|c|c|c|}
\hline & नें & 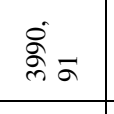 & $\begin{array}{l}\sigma \\
\end{array}$ & $\frac{a}{a}$ & & $f$ & 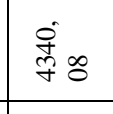 & $E$ & 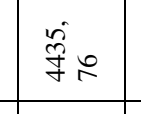 & 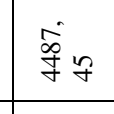 \\
\hline & $\sin$ & F & $\frac{a}{7} \pi$ & & & & $\frac{\overrightarrow{⿱ 亠 ⿰}}{\dot{y}}=$ & & & \\
\hline & 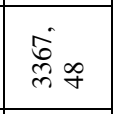 & 章素 & 政 & \% & & 等品 & 禁市 & of & \begin{tabular}{|l|}
$\frac{\infty}{b}$ \\
7
\end{tabular} & 妾: \\
\hline & 嘉 & $\frac{\delta}{\bar{y}}$ & 㚔多 & & & 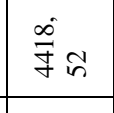 & 6 & \pm & t & 竞: \\
\hline & 冓 $_{\infty}$ & 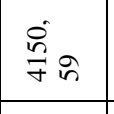 & 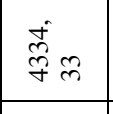 & & & & $\begin{array}{l}\text { 景 } \\
\end{array}$ & 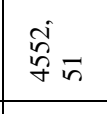 & & 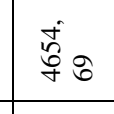 \\
\hline & $\frac{8}{9}$ & 装。 & 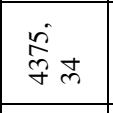 & & & & 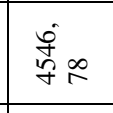 & \begin{tabular}{|l|l|} 
\\
\end{tabular} & & 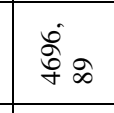 \\
\hline & 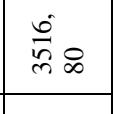 & 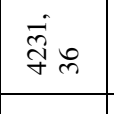 & $\begin{array}{l}0 \\
\frac{6}{y}\end{array}$ & 等= & & $\begin{array}{l}355 \\
\end{array}$ & 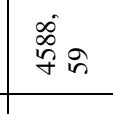 & $\begin{array}{l}\text { 尊品 } \\
\end{array}$ & 7 & + \\
\hline & 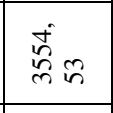 & 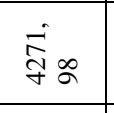 & 亲呆 & \begin{tabular}{|l|} 
等品 \\
\end{tabular} & & 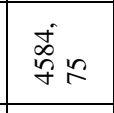 & $\begin{array}{l}\text { 产吉古 } \\
\end{array}$ & 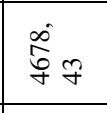 & 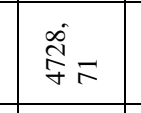 & 政 \\
\hline & 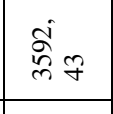 & 豙号: & 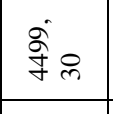 & & & & 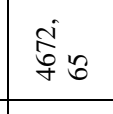 & 票下 & & 3 \\
\hline & 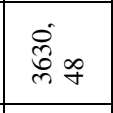 & 要。 & $\begin{array}{ll} \\
\\
3\end{array}$ & 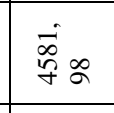 & & 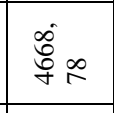 & $\begin{array}{l}\text { 孛。 } \\
\end{array}$ & 政士 & $\frac{38}{78}$ & 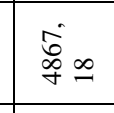 \\
\hline & 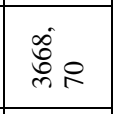 & 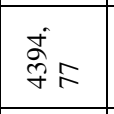 & 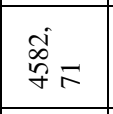 & $\frac{8}{9}$ & 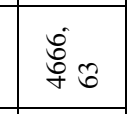 & 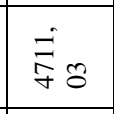 & 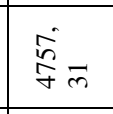 & 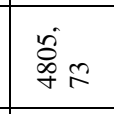 & 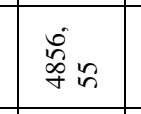 & 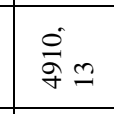 \\
\hline & 总: & 亲 & 莺声 & & & & 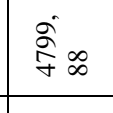 & of & & 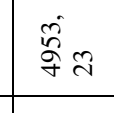 \\
\hline & 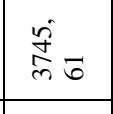 & 等守 & 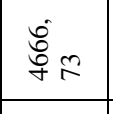 & & & & $\begin{array}{l}\text { 等品 } \\
\end{array}$ & 票品 & 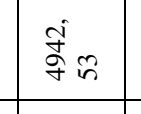 & 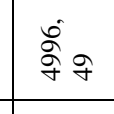 \\
\hline & 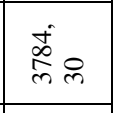 & 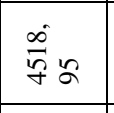 & 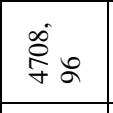 & 总: & To & 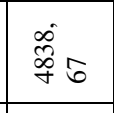 & 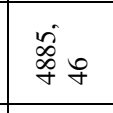 & 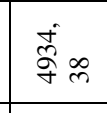 & 宷士 & 善2 \\
\hline & $\begin{array}{l}\mathbb{x}_{n} \\
\infty\end{array}$ & 钎古 & $\begin{array}{l}8 \\
8 \\
4\end{array}$ & $f^{2}$ & & 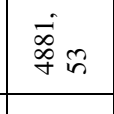 & 等要 & 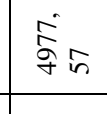 & $=$ & 罚: \\
\hline & 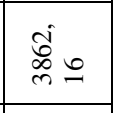 & 害品 & $\begin{array}{l}2 \\
q \\
q\end{array}$ & 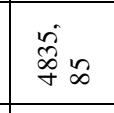 & & & 亭古 & 5 & 8 & 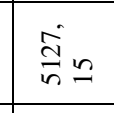 \\
\hline & 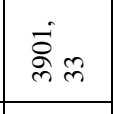 & 素严 & 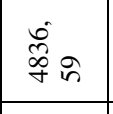 & +2 & & 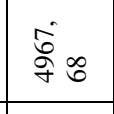 & 吉品 & 8 & $\frac{\circ}{\bar{n}}$ ब & + \\
\hline & 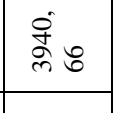 & 票。 & 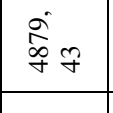 & 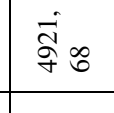 & $\frac{8}{9}$ & 㝘品 & 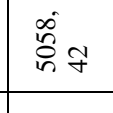 & $\frac{\mathscr{6}}{\bar{n}}$ & 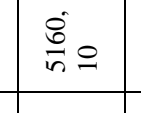 & 离: \\
\hline & 总 & 敞各 & 年궁 & 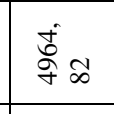 & | nk: & 管寺寺 & 咢方 & $\frac{5}{n} \infty$ & 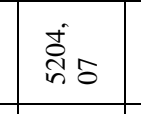 & 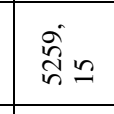 \\
\hline & & & & & & & & & & \\
\hline
\end{tabular}




\begin{tabular}{|c|c|c|c|c|c|c|c|c|c|c|}
\hline 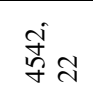 & 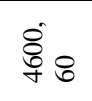 & هُ & $\begin{array}{l}\overrightarrow{\tilde{m}} 8 \\
f\end{array}$ & 窸 & 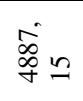 & 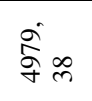 & 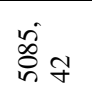 & 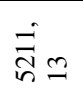 & 总品 & 点 \\
\hline 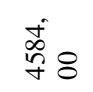 & 季 8 & 辛 & 点施 & 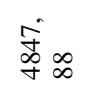 & 事上 & ิํำ & $\frac{\dot{\vec{d}}}{n}=$ & 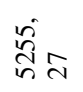 & 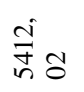 & 总 \\
\hline 产す & 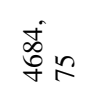 & $\begin{array}{l}\dot{y}+\infty \\
\vec{f} \infty\end{array}$ & $\begin{array}{l}\dot{0} \\
\text { on } \\
\text { on }\end{array}$ & 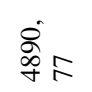 & 孚志 & 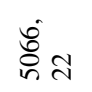 & $\begin{array}{l}\hat{N} \\
\hat{n}\end{array}$ & 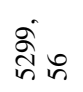 & 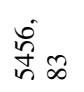 & $\begin{array}{l}\infty \\
\text { : } \\
\text { in }\end{array}$ \\
\hline 它 & 豙号 & $\begin{array}{l}\text { \&ें } \\
\text { के }\end{array}$ & \begin{tabular}{l}
$\infty$ \\
$\infty$ \\
ò \\
\multirow{\alpha}{*}{$\sigma$}
\end{tabular} & 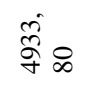 & 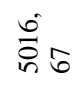 & $\frac{\partial}{i n}$ & iิ่8 & 趂 & 离品 & $\underset{i}{\stackrel{i}{i} \infty}$ \\
\hline$\stackrel{\dot{8}}{\frac{8}{4}}$ & $\begin{array}{l}\dot{8} \\
\dot{\sigma}\end{array}$ & 㣻 & 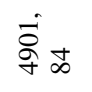 & $\begin{array}{l}\text { 字 } \\
\text { gे }\end{array}$ & 密 & $\frac{n}{n} 8$ & $\overrightarrow{\vec{D}} n$ & $\begin{array}{l}\infty \\
\infty \\
i n n \\
i n\end{array}$ & 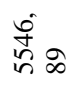 & 总 \\
\hline 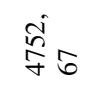 & 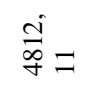 & $\begin{array}{l}\text { r. } \\
\text { os } 8\end{array}$ & $\begin{array}{l}\text { 焘 } \\
\text { 万人 }\end{array}$ & 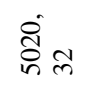 & 突。 & iे & 窟o & 勇品 & 令士 & $\begin{array}{l}\stackrel{0}{0} \\
\text { i. }\end{array}$ \\
\hline$\stackrel{\ddot{q}}{\vec{\gamma}} \overrightarrow{\mathrm{v}}$ & 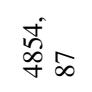 & $\begin{array}{l}\infty \\
\stackrel{\infty}{\sigma} \infty\end{array}$ & $\begin{array}{l}\infty \\
\stackrel{\circ}{\circ} \pm \\
\stackrel{\sigma}{\sigma} \pm\end{array}$ & 离 & 守的 & $\dot{\vec{d}} \vec{b}$ & $\begin{array}{l}\dot{8} \\
\text { in }\end{array}$ & 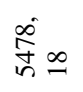 & 密的 & $\begin{array}{l}\hat{i} \\
\infty \\
\infty\end{array}$ \\
\hline 容 & $\stackrel{\stackrel{\circ}{\circ} \infty}{\stackrel{\infty}{a}}$ & 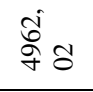 & $\dot{\vec{\rho}} \vec{n}$ & $\stackrel{\overrightarrow{0}}{n}$ & $\overrightarrow{\vec{\sigma}^{2}}$ & $\begin{array}{l}\dot{0} \\
\tilde{N}^{2}\end{array}$ & 离 & तี & 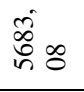 & $\begin{array}{l}\infty \\
\infty^{0} \\
n^{\circ}\end{array}$ \\
\hline 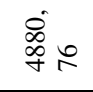 & 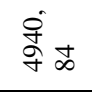 & 总 & 令 & $\overrightarrow{\vec{n}} \vec{n}$ & 疍 & 疍 & 害家 & 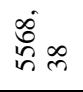 & \begin{tabular}{l}
$\dot{\infty}$ \\
\multirow{n}{*}{$\mathrm{n}$}
\end{tabular} & $\begin{array}{l}\text { 密 } \\
\text { in }\end{array}$ \\
\hline 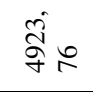 & 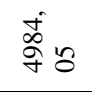 & 离 & $\stackrel{\infty}{=}$ & $\begin{array}{l}\dot{2} \\
\stackrel{n}{n} 0\end{array}$ & 空士 & î & 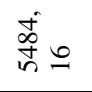 & 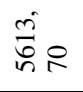 & 点 8 & ì \\
\hline 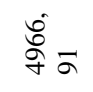 & 灾 & ถิ่ & $\begin{array}{l}\text { di } \\
\text { in }\end{array}$ & तें & 总= & 产出 & हैं & 总= & 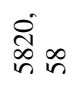 & 产。 \\
\hline$\stackrel{\circ}{\bar{D}} \overline{\mathrm{N}}$ & 宫 & $\begin{array}{l}0 \\
\stackrel{5}{n} 8\end{array}$ & 离员 & 令子 & 总 & 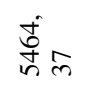 & 点 & 营市 & 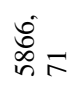 & $\begin{array}{l}\text { D. } \\
\text { ô: }\end{array}$ \\
\hline 胳。 & $\stackrel{\dot{J}}{\vec{n} \infty}$ & $\dot{\vec{n}}$ & $\begin{array}{l}\text { में } \\
\text { in }\end{array}$ & तi & mi & 商吕 & 产方 & 㤐苔 & ㄱํำ & $\overrightarrow{\vec{m}} \bar{i}$ \\
\hline 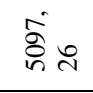 & $\begin{array}{l}\infty \\
n_{n}^{0} \\
n\end{array}$ & శึ่๋ & 离ळ & $\begin{array}{l}i \\
i \tilde{n} \\
i\end{array}$ & 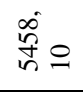 & $\begin{array}{l}\text { 点 } \\
\text { in }\end{array}$ & 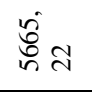 & 离ま & 拿守 & $\begin{array}{l}\infty \\
\stackrel{\infty}{\Delta} \infty \\
\end{array}$ \\
\hline $\overrightarrow{\vec{f}} \vec{b}$ & 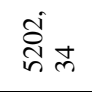 & $\begin{array}{l}\infty \\
\tilde{o}_{0}^{\circ} \pm\end{array}$ & हें & 离8 & 触8 & 鸽造 & 官 & $\begin{array}{l}\text { 季 } \\
\text { in }\end{array}$ & 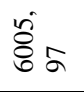 & तुำ \\
\hline$\frac{d}{\infty} \bar{a}$ & 导 & $\frac{i}{\tilde{n}}$ q & $\begin{array}{l}\infty \\
\infty \\
i n \infty \\
n \rightarrow \infty\end{array}$ & $\begin{array}{l}\overrightarrow{0}+\infty \\
\vec{b}+\infty\end{array}$ & 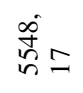 & 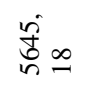 & 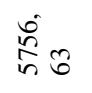 & $\begin{array}{c}\infty \\
\infty \\
\infty \\
i \\
i\end{array}$ & 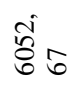 & 今̂d \\
\hline $\begin{array}{l}\text { तี่ } \\
\text { ชิ }\end{array}$ & 㤐下 & 岕に & $\begin{array}{l}\text { 学 } \\
\text { in in }\end{array}$ & 怘。 & 敌子 & 商主 & 容 & مू & gi & ซี้ส \\
\hline 空气 & 离 & 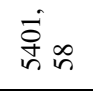 & 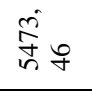 & 㫄去 & 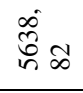 & $\begin{array}{l}\text { s. } \\
\text { in o }\end{array}$ & 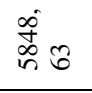 & 票的 & 它 & 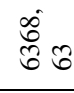 \\
\hline 鬲的 & î & 热 & $\begin{array}{l}\infty \\
\text { 离 } \\
\text { f }\end{array}$ & 㤐古 & $\begin{array}{l}\text { 我 } \\
\text { 总的 }\end{array}$ & $\begin{array}{l}i \\
i \\
i \\
i n\end{array}$ & 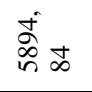 & 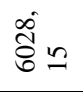 & 今̊ & F \\
\hline$\simeq$ & $=$ & $\cong$ & 0 & $\infty$ & & $\rho$ & . & + & $m$ & \\
\hline
\end{tabular}




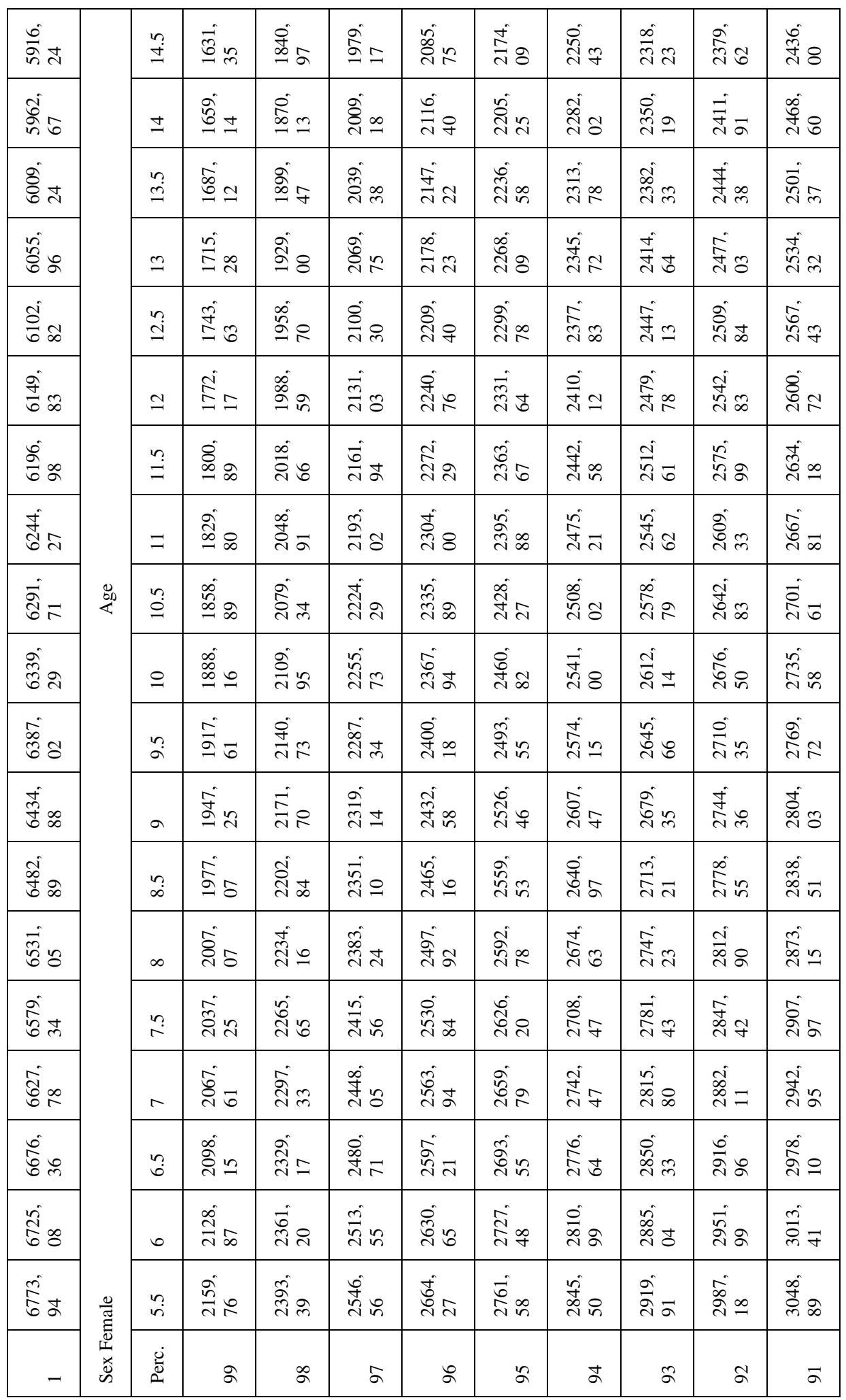




\begin{tabular}{|c|c|c|c|c|c|c|c|c|c|c|}
\hline $\begin{array}{l}\infty \\
\vdots \\
d \\
d\end{array}$ & 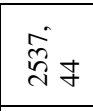 & 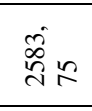 & हู่ส & 逭 & 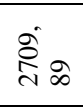 & 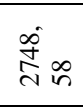 & $\begin{array}{l}w \\
\infty \\
i \\
\sim\end{array}$ & 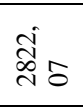 & 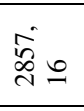 & 离 त \\
\hline स्तुં & $\begin{array}{l}\dot{8} \\
\text { तi }\end{array}$ & 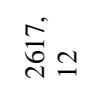 & $\overrightarrow{\vec{d}} \overrightarrow{\mathrm{d}} \overline{\mathrm{m}}$ & 炃 & 疍。 & $\begin{array}{l}\dot{1} \\
\substack{\infty \\
\sim}\end{array}$ & 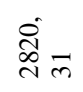 & 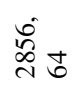 & 愈 & ลั่ \\
\hline 苫 & 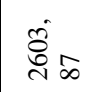 & : & 悹 & तेंo & $\stackrel{\infty}{\stackrel{\infty}{*} \infty}$ & 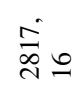 & 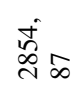 & $\overrightarrow{\vec{D}} \dot{d} \tilde{m}$ & ลั่ & م. \\
\hline $\begin{array}{l}\text { 离 } \\
\text { तें }\end{array}$ & 莺品 & 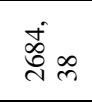 & సิ่ & 离可 & 敨学 & 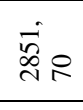 & 总 & ปัँ & 离离 & 离。 \\
\hline 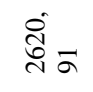 & 灾8 & $\stackrel{\infty}{\stackrel{\infty}{*}}$ & 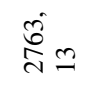 & 岕 & 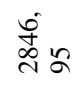 & 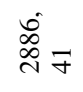 & 苛 & 离 & 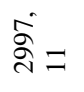 & 总。 \\
\hline 晜子 & $\stackrel{\sim}{a}_{\vec{N}}^{+}$ & $\begin{array}{l}\hat{v} \\
\text { ลें }\end{array}$ & 今े & 总 F & 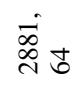 & ลี่ & 突㶽 & 究莴 & हृं & $\dot{\vec{s}}_{\tilde{D}}$ \\
\hline $\begin{array}{l}\infty \\
0 \\
\overbrace{}^{\circ}\end{array}$ & $\begin{array}{l}\infty \\
\overbrace{\Lambda}^{\infty} \infty\end{array}$ & 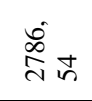 & $\overrightarrow{\mathbb{N}_{\infty}^{0} \infty}$ & $\begin{array}{l}\text { r. } \\
\text { do } \\
\text { dis }\end{array}$ & 突 & 离 & 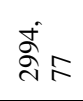 & 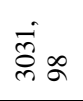 & 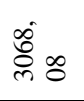 & $\stackrel{\text { }}{\mathrm{m}} \mathrm{d}$ \\
\hline ત્ત & 究品 & 总 & ơ⿱ & 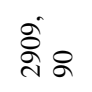 & $\begin{array}{l}\overrightarrow{5} \\
\text { îं in }\end{array}$ & 䓫苛 & 总= & 密 & 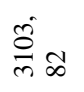 & $\stackrel{\vec{m}}{m} \circ$ \\
\hline 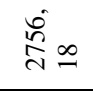 & 客 & 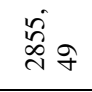 & $\overrightarrow{\overrightarrow{\mathrm{C}}}+\overrightarrow{\mathrm{N}}$ & 表主 & ڤั & 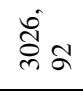 & 客 & 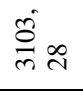 & 商 & $\stackrel{v}{\stackrel{0}{m}}$ \\
\hline 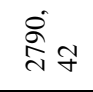 & 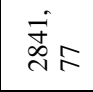 & 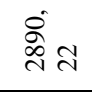 & ๙ึ் & $\begin{array}{l}\dot{\infty} \\
\text { aे t }\end{array}$ & ฮิ่ \& & ơ & 产字 & $\stackrel{\vec{\rho}}{\vec{m}} \underline{\sim}$ & 点 & $\dot{\vec{\sim}} \vec{~}$ \\
\hline 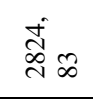 & 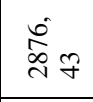 & ลิ่ & $\overrightarrow{\text { तें }}$ & 突离的 & 总冓 & $\begin{array}{l}\infty \\
D^{\circ}=\end{array}$ & $\stackrel{\vec{m}}{\vec{m}}$ & $\stackrel{\text { r. }}{\vec{m}}$ & 귤 & $\begin{array}{l}\dot{f} \\
\dot{m} \infty\end{array}$ \\
\hline $\begin{array}{l}\stackrel{0}{0} \\
\stackrel{\Delta}{\sim}\end{array}$ & $\overrightarrow{\vec{\nabla}}$ & $\begin{array}{l}0 \\
\stackrel{\circ}{\circ} \infty\end{array}$ & 总 & 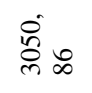 & 密ה & 点 & 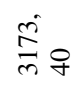 & $\overrightarrow{\vec{J}}$ 子 & 怨子 & 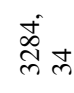 \\
\hline 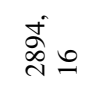 & هơ & $\begin{array}{l}\text { ڤे } \\
\text { aेf }\end{array}$ & 㝘施 & $\begin{array}{l}\text { D. } \\
\mathbb{e}^{\circ} \bar{n}\end{array}$ & गิ & $\stackrel{8}{\stackrel{8}{m} 8}$ & बें & 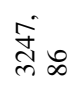 & 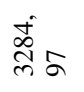 & $\overrightarrow{\tilde{N}}$ \\
\hline ลิ่ & $\begin{array}{l}\overrightarrow{0} \\
\overrightarrow{\mathrm{d}} \\
\text { f }\end{array}$ & 芯ळ & 今. & $\frac{\vec{d}}{m}{ }_{m}$ & $\stackrel{8}{\stackrel{8}{m}} \pm$ & ث્లై & 串8 & 㞼 & लై & 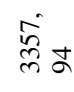 \\
\hline 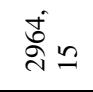 & : & 总 & $\stackrel{m}{\Rightarrow} g$ & $\begin{array}{l}\infty \\
\stackrel{\infty}{m} \\
m\end{array}$ & $\overrightarrow{\overrightarrow{\tilde{N}}}$ & సี่ & 焉 & $\overrightarrow{\vec{j}} \pm$ & $\begin{array}{l}\infty \\
\aleph_{\infty}^{\infty} \infty \\
\overbrace{n}^{\infty}\end{array}$ & 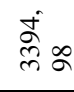 \\
\hline बूें & 令 & $\frac{8}{m} 8$ & $\stackrel{\vec{g}}{m}_{\infty}^{\infty}$ & $\stackrel{+}{\stackrel{5}{m}}$ & लें & बेंন & बेंల & 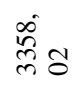 & 躎 & 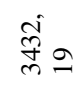 \\
\hline 总 & $\begin{array}{l}\overrightarrow{0} \\
\stackrel{\rho}{\infty} \sigma\end{array}$ & ma & $\stackrel{\infty}{m} \frac{\infty}{m} g$ & స్లి & 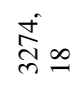 & $\stackrel{r}{p}_{\infty} \infty$ & 岕 & 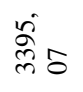 & 话 & 总。 \\
\hline 㝘付 & $\frac{\vec{d}}{m}+$ & $\stackrel{+}{\stackrel{m}{m}}$ & 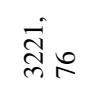 & $\overrightarrow{\tilde{d}}$ & $\stackrel{\circ}{\bar{p}_{\infty}} \infty$ & हี่ & 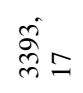 & 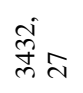 & 京 & 离 \\
\hline$\stackrel{8}{\stackrel{8}{m} \pm}$ & 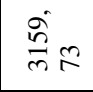 & $\stackrel{\vec{N}}{\vec{N}}$ & $\begin{array}{l}\infty \\
\text { సె่ }\end{array}$ & 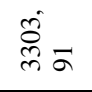 & 熟。 & $\begin{array}{l}\text { बi. } \\
\text { din }\end{array}$ & 害品 & 总 & 总士 & 恖 \\
\hline 8 & $\infty$ & $\infty$ & 2 & $\infty$ & $\infty$ & 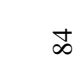 & $\infty$ & $\infty$ & $\vec{\infty}$ & \\
\hline
\end{tabular}




\begin{tabular}{|c|c|c|c|c|c|c|c|c|}
\hline & & 释= & 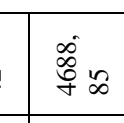 & & है। & 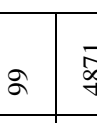 & & \\
\hline & 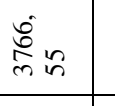 & 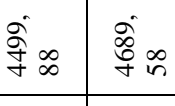 & $\overrightarrow{\underline{s}}$ & & 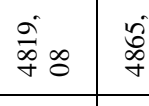 & & & \\
\hline & 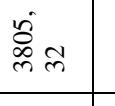 & & 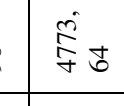 & & & $\{=$ & & \\
\hline & 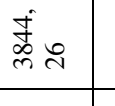 & & $\frac{6}{4}$ & & & & & \\
\hline & 細 & 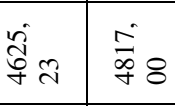 & 3 & & $\frac{7}{7 \infty}$ & & & \\
\hline & ฐ & 細 & $=\frac{1}{3}$ & 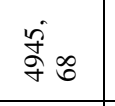 & 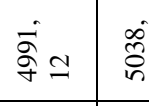 & & & \\
\hline & 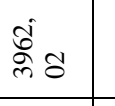 & 等。 & 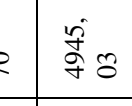 & 橧。 & 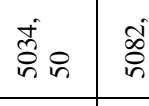 & 8 & & \\
\hline & 紊 & $\begin{array}{l}5 \% \\
7\end{array}$ & $=\frac{y_{3}}{3}$ & si & $\frac{6}{y_{n}^{\prime}}$ & & & 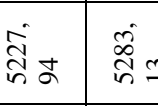 \\
\hline & 亨 & 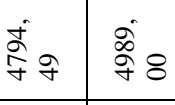 & $=\frac{\vec{g}}{\vec{n}}$ & $\vec{x}_{\mid}$ & 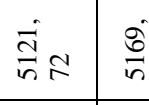 & 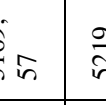 & & $8 \pm$ \\
\hline & 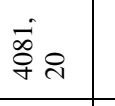 & 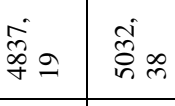 & 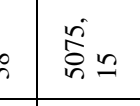 & 容手 & $\underline{n}$ & $\approx$ & & 藏等 \\
\hline & 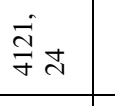 & 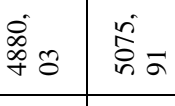 & $\frac{\mathscr{x}}{\bar{n}}=$ & $=$ & 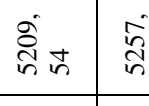 & & & \\
\hline & 章事 & 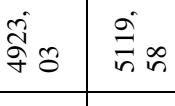 & $\frac{d}{n g}$ & 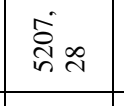 & : & 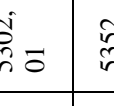 & & \\
\hline & & $\bar{F}$ & $=$ & 看 & 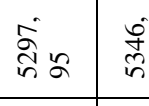 & & & \\
\hline & 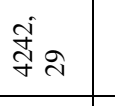 & 竞品 & 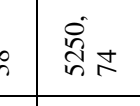 & : & 吾 & 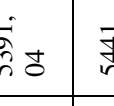 & & \\
\hline & 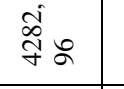 & 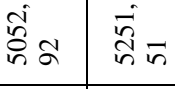 & 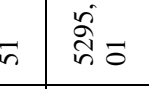 & 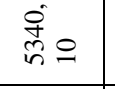 & 总: & & & \\
\hline & 7 & 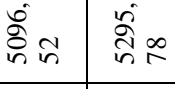 & 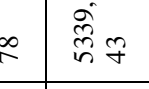 & $m$ & 覈 & 尊 & & \\
\hline & $\neq i$ & 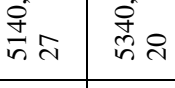 & 8 & 곰요 & $\mathscr{n}$ & So & & \\
\hline & 繁市 & 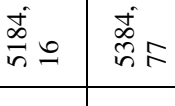 & 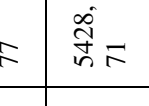 & & 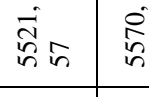 & & & \\
\hline & & "ृ. & 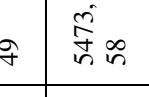 & $\bar{n} \bar{n}=1$ & 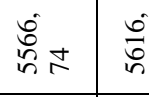 & & & \\
\hline & & & & & & & & \\
\hline
\end{tabular}




\begin{tabular}{|c|c|c|c|c|c|c|c|c|c|c|}
\hline & & 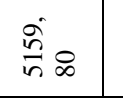 & 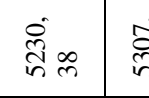 & & & & & & 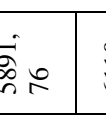 & \\
\hline & 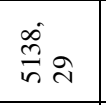 & 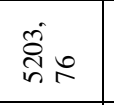 & & & & & & & 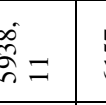 & \\
\hline & & & & & & & 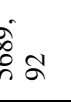 & 8 & 咅。 & \\
\hline & & & & & & & & & & \\
\hline & 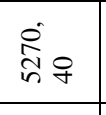 & & & & & & & & 8 & \\
\hline & & & & & & & & & 58 & \\
\hline & & 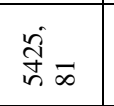 & 雱合 & & & & & & 68 & \\
\hline & $\frac{5}{5}$ & 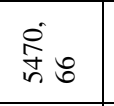 & 密市 & & & & & & 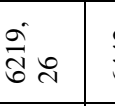 & \\
\hline & & & & 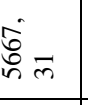 & & & 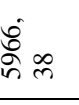 & $\frac{8}{8}$ & 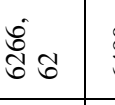 & \\
\hline & 票品 & 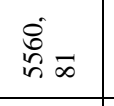 & & & & & $\overline{8} 8$ & 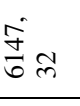 & 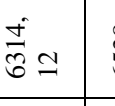 & \\
\hline & 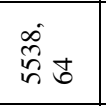 & 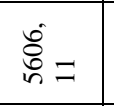 & 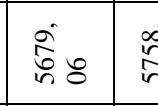 & 㓩士 & $\pi$ & 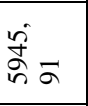 & 8 & $\frac{8}{67} 7$ & 8 & \\
\hline & & 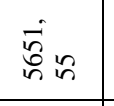 & 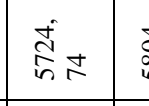 & 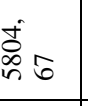 & 68 & 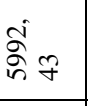 & 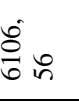 & 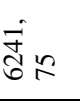 & 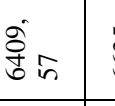 & \\
\hline & 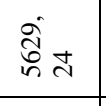 & 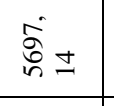 & 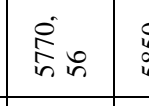 & 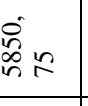 & & 管里 & $\underbrace{\prime}_{0}$ & $\underline{\simeq}$ & $\begin{array}{l}2 \\
z\end{array}$ & 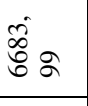 \\
\hline & 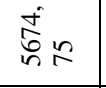 & 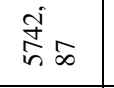 & 紊安 & & & & & 6 & 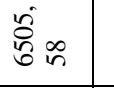 & \\
\hline & 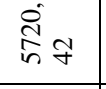 & 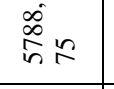 & 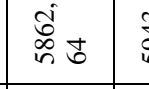 & $f=$ & $8 \pi$ & $5 x$ & & $\frac{6}{67}$ & 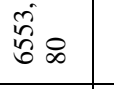 & \\
\hline & 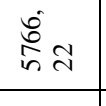 & 繯 & 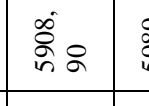 & $x^{2} \infty$ & $\begin{array}{l}50 \\
\end{array}$ & 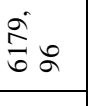 & 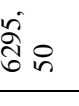 & $\therefore$ & 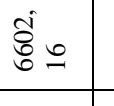 & \\
\hline & 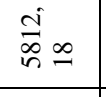 & & & & & & & & & \\
\hline & 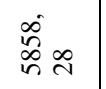 & 8 & 5 & & & & & & 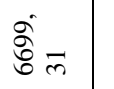 & \\
\hline & 吉品 & 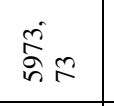 & 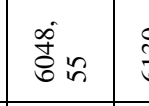 & & 68 & $8=$ & & & & \\
\hline & & & & & & & & & & \\
\hline
\end{tabular}




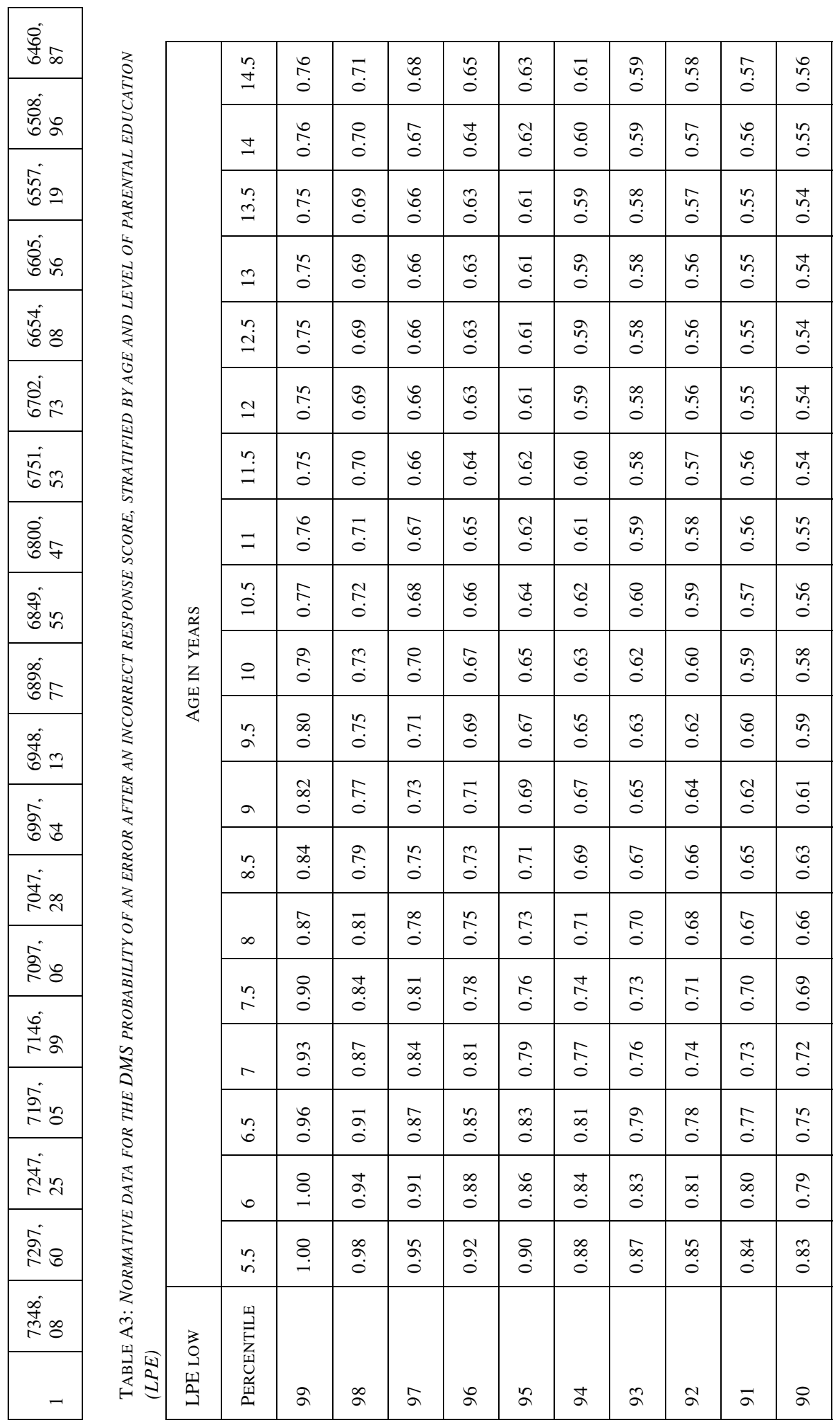




\begin{tabular}{|c|c|c|c|c|c|c|c|c|c|c|c|c|c|c|}
\hline$\stackrel{+}{n}$ & $\tilde{n}$ & $\stackrel{n}{n}$ & $\stackrel{n}{n}$ & $\stackrel{n}{0}$ & مי & 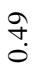 & $\stackrel{\infty}{\stackrel{\infty}{0}}$ & $\stackrel{\infty}{\stackrel{\infty}{0}}$ & $\stackrel{f}{+}$ & $\stackrel{?}{\stackrel{0}{\circ}}$ & లి & $\stackrel{0}{\stackrel{0}{0}}$ & $\stackrel{m}{\circ}$ & $\stackrel{2}{\circ}$ \\
\hline$\stackrel{n}{n}$ & $\stackrel{n}{n}$ & $\stackrel{n}{n}$ & $\tilde{n}$ & ஜn & $\stackrel{g}{\sigma}$ & 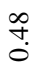 & f̊ & f & $\begin{array}{l}0 \\
\stackrel{0}{0}\end{array}$ & $\stackrel{\mathscr{P}}{\stackrel{0}{0}}$ & ָे & $\stackrel{0}{\stackrel{0}{0}}$ & $\frac{N}{0}$ & $\exists$ \\
\hline 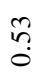 & ñ & $\tilde{n}$ & $\stackrel{n}{n}$ & 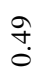 & 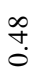 & $\stackrel{f}{0}$ & f̊. & 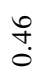 & $\stackrel{n}{\stackrel{n}{0}}$ & $\stackrel{\text { I }}{0}$ & \begin{tabular}{l}
$\infty$ \\
\multirow{0}{0}{} \\
0
\end{tabular} & $\frac{n}{0}$ & $\frac{1}{0}$ & $\overline{0}$ \\
\hline$\stackrel{n}{n}$ & $\tilde{n}$ & $\tilde{n}$ & $\stackrel{n}{0}$ & $\stackrel{\stackrel{q}{+}}{\circ}$ & $\stackrel{\infty}{\stackrel{0}{0}}$ & †े & $\begin{array}{l}0 \\
\stackrel{0}{0} \\
0\end{array}$ & $\begin{array}{l}0 \\
\stackrel{0}{0}\end{array}$ & $\stackrel{n}{\stackrel{n}{0}}$ & $\stackrel{\text { I }}{0}$ & 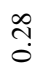 & $\stackrel{n}{\circ}$ & $\exists$ & $\stackrel{0}{\circ}$ \\
\hline$\stackrel{n}{n}$ & $\bar{n}$ & $\tilde{n}$ & $\stackrel{n}{0}$ & $\stackrel{\stackrel{q}{0}}{\circ}$ & $\stackrel{\infty}{\stackrel{0}{0}}$ & †े & $\begin{array}{l}0 \\
\stackrel{0}{0} \\
0\end{array}$ & $\begin{array}{l}0 \\
\stackrel{0}{0}\end{array}$ & $\stackrel{?}{\stackrel{n}{0}}$ & $\underset{0}{\vec{J}}$ & 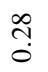 & $\stackrel{ \pm}{\stackrel{0}{0}}$ & $\exists$ & $\stackrel{0}{\circ}$ \\
\hline$\stackrel{n}{n}$ & $\tilde{n}$ & $\ddot{n}$ & $\stackrel{n}{0}$ & 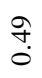 & $\stackrel{\infty}{+\infty}$ & $\stackrel{f}{\circ}$ & f̊. & 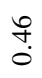 & $\stackrel{n}{\stackrel{n}{0}}$ & $\stackrel{\text { I }}{0}$ & $\stackrel{\substack{n \\
0}}{0}$ & $\stackrel{n}{\stackrel{0}{0}}$ & $\overline{0}$ & $\overline{0}$ \\
\hline$\stackrel{n}{n}$ & $\tilde{n}$ & $\tilde{n}$ & $\stackrel{n}{0}$ & $\stackrel{\stackrel{q}{+}}{\circ}$ & $\stackrel{g}{\sigma}$ & $\stackrel{\infty}{+\infty}$ & f̊ & $\begin{array}{l}0 \\
0 \\
0\end{array}$ & $\begin{array}{l}0 \\
\stackrel{0}{0}\end{array}$ & $\stackrel{\text { I }}{0}$ & ָे & $\frac{n}{0}$ & $\frac{1}{0}$ & $\exists$ \\
\hline$\stackrel{+}{n}$ & $\ddot{n}$ & $\stackrel{n}{n}$ & "ñ & ?̊ & $\stackrel{g}{\stackrel{\sigma}{\circ}}$ & $\stackrel{g}{+}$ & $\stackrel{\infty}{\stackrel{\infty}{0}}$ & f & $\begin{array}{l}0 \\
\stackrel{0}{0}\end{array}$ & $\stackrel{?}{\stackrel{0}{0}}$ & ֻे & $\stackrel{\circ}{\stackrel{0}{0}}$ & $\stackrel{m}{0}$ & $\frac{1}{0}$ \\
\hline$n$ & 華 & $\tilde{n}$ & $\stackrel{n}{n}$ & $\tilde{n}$ & $\bar{n}$ & ?̊ & $\underset{\stackrel{g}{q}}{\stackrel{g}{0}}$ & $\stackrel{\infty}{+}$ & $\stackrel{f}{0}$ & $\underset{0}{\sharp}$ & $\overrightarrow{0}$ & $\frac{1}{0}$ & $\frac{ \pm}{0}$ & $\frac{m}{0}$ \\
\hline$n$ & ñ & $\begin{array}{l}n \\
n \\
0\end{array}$ & $\stackrel{+}{n}$ & $\stackrel{n}{n}$ & $\tilde{n}$ & $\widetilde{n}$ & $\stackrel{n}{n}$ & $\begin{array}{l}\text { ? } \\
0\end{array}$ & 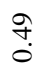 & $\stackrel{n}{\stackrel{n}{0}}$ & $\stackrel{\tilde{c}}{0}$ & $\frac{9}{0}$ & $\frac{n}{0}$ & $\stackrel{ \pm}{0}$ \\
\hline$\stackrel{\infty}{n}$ & $\tilde{n}$ & $\begin{array}{l}n \\
? \\
0\end{array}$ & $\stackrel{n}{n}$ & $\stackrel{+}{n}$ & $\stackrel{+}{n}$ & $\stackrel{n}{n}$ & $\stackrel{1}{n}$ & $\tilde{n}$ & \begin{tabular}{l}
$n$ \\
\hdashline \\
0
\end{tabular} & f̊ & $\stackrel{m}{0}$ & ণั & $\frac{1}{0}$ & $\frac{0}{0}$ \\
\hline $\begin{array}{l}\stackrel{8}{0} \\
\dot{0}\end{array}$ & ñ & $\begin{array}{l}\infty \\
n \\
0\end{array}$ & ñ & $\begin{array}{l}n \\
? \\
0\end{array}$ & $\begin{array}{l}n \\
? \\
0\end{array}$ & $\stackrel{n}{n}$ & $\stackrel{+}{n}$ & $\ddot{n}$ & $\stackrel{n}{n}$ & $\stackrel{g}{+}$ & $\stackrel{?}{0}$ & $\stackrel{\text { İ }}{0}$ & $\frac{9}{0}$ & $\frac{\infty}{0}$ \\
\hline $\begin{array}{l}\hat{b} \\
\stackrel{0}{0}\end{array}$ & $\begin{array}{l}\overline{0} \\
\dot{0}\end{array}$ & $\begin{array}{l}\stackrel{0}{0} \\
0\end{array}$ & $\stackrel{n}{0}$ & ñ? & $\begin{array}{l}\infty \\
? \\
0\end{array}$ & $\tilde{n}$ & \begin{tabular}{l}
$\stackrel{0}{n}$ \\
\hdashline
\end{tabular} & $\stackrel{n}{n}$ & $\stackrel{n}{n}$ & $\tilde{n}$ & $\stackrel{\infty}{\dddot{0}}$ & $\stackrel{\text { I }}{0}$ & $\vec{\jmath}$ & ָे \\
\hline $\begin{array}{l}n \\
b \\
0\end{array}$ & $\begin{array}{l}\dot{J} \\
\dot{0}\end{array}$ & $\begin{array}{l}\tilde{b} \\
0\end{array}$ & $\begin{array}{l}\mathcal{b} \\
\stackrel{0}{0}\end{array}$ & $\underset{\sigma}{\sigma}$ & $\begin{array}{l}8 \\
\stackrel{0}{0}\end{array}$ & กิ? & 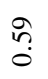 & $\begin{array}{l}\infty \\
\dddot{n} \\
0\end{array}$ & $\tilde{n}$ & ?ִ & $\stackrel{?}{\stackrel{0}{0}}$ & ஸ̃ & 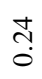 & $\stackrel{\overbrace{}}{\tilde{o}}$ \\
\hline $\begin{array}{l}\infty \\
\stackrel{0}{0} \\
\dot{0}\end{array}$ & $\begin{array}{l}\hat{\sigma} \\
0\end{array}$ & $\begin{array}{l}0 \\
\stackrel{0}{0}\end{array}$ & ?ֶ. & $\stackrel{t}{t}$ & $\underset{0}{\mathscr{0}}$ & $\begin{array}{l}\widetilde{\sigma} \\
0\end{array}$ & $\begin{array}{l}\tilde{b} \\
0\end{array}$ & $\underset{\sigma}{\sigma}$ & $\begin{array}{l}0 \\
0 \\
0\end{array}$ & $n$ & $\stackrel{\mathscr{P}}{\stackrel{0}{0}}$ & ֻ̊ & $\begin{array}{l}\text { N̦ } \\
0\end{array}$ & 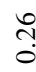 \\
\hline$\tilde{i}$ & $\stackrel{?}{\stackrel{0}{0}}$ & $\begin{array}{l}0 \\
0 \\
0\end{array}$ & $\begin{array}{l}\infty \\
\stackrel{0}{0} \\
0\end{array}$ & $\underset{0}{0}$ & \begin{tabular}{l}
0 \\
\hdashline \\
0
\end{tabular} & $\begin{array}{l}n \\
0 \\
0\end{array}$ & $\begin{array}{l}n \\
0 \\
0\end{array}$ & $\stackrel{t}{0}$ & $\stackrel{n}{0}$ & : & $\begin{array}{l}0 \\
\stackrel{0}{0} \\
0\end{array}$ & $\stackrel{m}{0}$ & $\stackrel{\text { ते }}{0}$ & 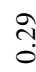 \\
\hline$\stackrel{t}{\stackrel{0}{0}}$ & $\stackrel{m}{\circ}$ & $\stackrel{N}{\stackrel{0}{0}}$ & $\tilde{\sigma}$ & $\stackrel{?}{\stackrel{0}{0}}$ & $\stackrel{?}{\stackrel{0}{0}}$ & $\begin{array}{l}0 \\
0 \\
0\end{array}$ & $\begin{array}{l}\infty \\
\stackrel{0}{0} \\
\dot{0}\end{array}$ & $\underset{0}{0}$ & $\hat{\sigma}$ & ?ో. & ?̊ & 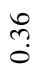 & $\stackrel{m}{0}$ & $\stackrel{?}{3}$ \\
\hline$\stackrel{\infty}{\stackrel{\infty}{0}}$ & $\stackrel{r}{0}$ & $\stackrel{0}{\stackrel{0}{0}}$ & $\stackrel{n}{\stackrel{0}{0}}$ & $\frac{t}{0}$ & $\stackrel{m}{\stackrel{0}{0}}$ & $\stackrel{\mathbb{2}}{\stackrel{0}{0}}$ & $\stackrel{1}{\stackrel{0}{0}}$ & $\overrightarrow{0}$ & $\stackrel{?}{\stackrel{0}{0}}$ & ک̊. & $\stackrel{n}{n}$ & $\stackrel{?}{\stackrel{0}{0}}$ & $\hat{n}$ & $\begin{array}{l}\text { ?ִ } \\
\text { ?n }\end{array}$ \\
\hline $\begin{array}{l}\stackrel{\infty}{\infty} \\
0 \\
0\end{array}$ & $\begin{array}{l}\bar{\infty} \\
0\end{array}$ & $\begin{array}{l}\infty \\
\stackrel{0}{0} \\
0\end{array}$ & $\stackrel{\overbrace{}}{\circ}$ & $\stackrel{\infty}{\stackrel{\infty}{0}}$ & $\stackrel{\hat{\imath}}{\hat{0}}$ & $\begin{array}{l}\stackrel{0}{0} \\
\vdots\end{array}$ & $\begin{array}{l}\stackrel{0}{0} \\
\vdots\end{array}$ & $\stackrel{n}{\mathfrak{0}}$ & $\stackrel{t}{i}$ & $\stackrel{\nabla}{0}$ & $\tilde{n}$ & $\underset{0}{\sharp}$ & $\underset{0}{*}$ & $\stackrel{o}{\stackrel{0}{0}}$ \\
\hline$\infty$ & $\infty$ & $\bar{\infty}$ & $\infty$ & 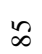 & $\underset{\infty}{ \pm}$ & 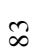 & $\infty$ & $\bar{\infty}$ & $\infty$ & $\stackrel{n}{2}$ & in & $\stackrel{\sim}{\sim}$ & ते & $\stackrel{2}{-}$ \\
\hline
\end{tabular}




\begin{tabular}{|c|c|c|c|c|c|c|c|c|c|c|c|c|c|c|}
\hline$\frac{1}{0}$ & $=$ & $\begin{array}{l}\stackrel{0}{0} \\
0\end{array}$ & $\stackrel{\circ}{\circ}$ & $\stackrel{\infty}{\stackrel{\circ}{0}}$ & ¿. & $\stackrel{\circ}{\circ}$ & $\stackrel{n}{\circ}$ & $\stackrel{t}{0}$ & $\stackrel{3}{0}$ & $\begin{array}{l}\Omega \\
\stackrel{0}{0}\end{array}$ & $\stackrel{8}{8}$ & $\stackrel{8}{8}$ & $\underset{0}{8}$ & $\stackrel{8}{8}$ \\
\hline$=$ & $\begin{array}{l}\stackrel{0}{0} \\
\stackrel{0}{0}\end{array}$ & $\begin{array}{l}a \\
\dot{0}\end{array}$ & $\stackrel{\infty}{\stackrel{\infty}{0}}$ & $\stackrel{0}{0}$ & ¿. & $\begin{array}{l}0 \\
\stackrel{0}{0}\end{array}$ & $\stackrel{n}{0}$ & $\stackrel{0}{0}$ & $\begin{array}{l}\tilde{O} \\
\stackrel{0}{0}\end{array}$ & $\stackrel{\overrightarrow{0}}{\dot{0}}$ & $\stackrel{8}{:}$ & $\stackrel{8}{:}$ & $\begin{array}{l}8 \\
\stackrel{0}{0}\end{array}$ & $\begin{array}{l}\stackrel{8}{0} \\
\stackrel{0}{0}\end{array}$ \\
\hline$\stackrel{\circ}{\circ}$ & $\stackrel{\stackrel{\partial}{\partial}}{\circ}$ & $\stackrel{8}{\circ}$ & $\stackrel{\infty}{\stackrel{\infty}{0}}$ & $\stackrel{0}{0}$ & $\stackrel{\circ}{\circ}$ & $\stackrel{n}{\circ}$ & $\stackrel{+}{0}$ & $\stackrel{m}{0}$ & 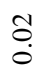 & $\stackrel{8}{\circ}$ & $\stackrel{8}{\circ}$ & $\stackrel{8}{\circ}$ & $\stackrel{8}{\circ}$ & $\stackrel{8}{\circ}$ \\
\hline$\stackrel{\circ}{\circ}$ & $\begin{array}{l}\stackrel{g}{0} \\
\stackrel{0}{0}\end{array}$ & $\begin{array}{l}\infty \\
\stackrel{0}{0}\end{array}$ & $\stackrel{5}{0}$ & $\stackrel{\bullet}{\circ}$ & $\stackrel{\leftrightarrow}{\circ}$ & $\stackrel{n}{0}$ & $\stackrel{t}{0}$ & 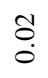 & $\stackrel{\overrightarrow{0}}{\circ}$ & $\stackrel{8}{\circ}$ & $\stackrel{8}{\circ}$ & $\stackrel{8}{\circ}$ & $\stackrel{8}{\circ}$ & $\stackrel{8}{\circ}$ \\
\hline$\stackrel{\circ}{\circ}$ & $\begin{array}{l}\stackrel{g}{0} \\
\dot{0}\end{array}$ & $\begin{array}{l}\infty \\
\stackrel{0}{0}\end{array}$ & $\stackrel{5}{0}$ & $\stackrel{8}{\circ}$ & $\stackrel{n}{\circ}$ & $\stackrel{t}{\dot{D}}$ & $\stackrel{n}{0}$ & 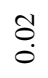 & $\stackrel{\overrightarrow{0}}{\circ}$ & $\stackrel{8}{\circ}$ & $\stackrel{8}{\circ}$ & $\stackrel{8}{\circ}$ & $\stackrel{8}{\circ}$ & $\stackrel{8}{\circ}$ \\
\hline$\stackrel{\circ}{\circ}$ & $\begin{array}{l}\stackrel{g}{0} \\
\stackrel{0}{0}\end{array}$ & $\stackrel{\infty}{\stackrel{\infty}{0}}$ & $\stackrel{0}{0}$ & $\stackrel{0}{0}$ & $\stackrel{\leftrightarrow}{\circ}$ & $\stackrel{n}{o}$ & $\stackrel{t}{0}$ & $\stackrel{m}{0}$ & $\stackrel{\overrightarrow{0}}{\circ}$ & $\stackrel{8}{\circ}$ & $\stackrel{8}{\circ}$ & $\stackrel{8}{\circ}$ & $\stackrel{8}{\circ}$ & $\stackrel{8}{\circ}$ \\
\hline$\stackrel{\circ}{\circ}$ & $\stackrel{\circ}{\circ}$ & $\stackrel{8}{\circ}$ & $\stackrel{\infty}{\circ}$ & $\stackrel{0}{0}$ & $\stackrel{\leftrightarrow}{\circ}$ & $\stackrel{n}{o}$ & $\stackrel{t}{0}$ & $\stackrel{m}{0}$ & 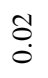 & $\stackrel{0}{0}$ & $\stackrel{8}{\circ}$ & $\stackrel{8}{\circ}$ & $\stackrel{8}{\circ}$ & $\stackrel{8}{\circ}$ \\
\hline$\vec{J}$ & $\stackrel{\circ}{\circ}$ & $\stackrel{0}{\circ}$ & $\stackrel{\circ}{\circ}$ & $\stackrel{\infty}{\stackrel{\infty}{0}}$ & ¿̊. & $\stackrel{\circ}{\circ}$ & $\stackrel{n}{\circ}$ & $\stackrel{t}{0}$ & $\stackrel{n}{0}$ & $\stackrel{\sigma}{\circ}$ & $\stackrel{8}{\circ}$ & $\stackrel{8}{\circ}$ & $\stackrel{8}{\circ}$ & $\stackrel{8}{\circ}$ \\
\hline$\frac{1}{0}$ & $\frac{1}{0}$ & $\exists$ & $\stackrel{0}{\stackrel{0}{0}}$ & $\stackrel{8}{\circ}$ & $\stackrel{\infty}{\circ}$ & $\stackrel{\circ}{\circ}$ & $\stackrel{\leftrightarrow}{\circ}$ & $\stackrel{\leftrightarrow}{\circ}$ & $\stackrel{t}{0}$ & 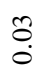 & $\stackrel{\sigma}{0}$ & $\stackrel{8}{\circ}$ & $\stackrel{8}{\circ}$ & $\stackrel{8}{\circ}$ \\
\hline$\frac{ \pm}{0}$ & $\stackrel{m}{0}$ & $\frac{1}{0}$ & $\exists$ & $\stackrel{0}{\stackrel{0}{0}}$ & $\stackrel{\circ}{\circ}$ & $\stackrel{\infty}{\stackrel{\infty}{0}}$ & $\stackrel{1}{0}$ & $\stackrel{\circ}{\circ}$ & $\stackrel{n}{0}$ & $\stackrel{t}{0}$ & 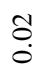 & $\stackrel{\Xi}{0}$ & $\stackrel{8}{\circ}$ & $\stackrel{8}{\circ}$ \\
\hline$\stackrel{n}{\circ}$ & $\frac{n}{0}$ & $\stackrel{ \pm}{\circ}$ & $\stackrel{m}{\circ}$ & $\stackrel{\sim}{\stackrel{0}{0}}$ & $=$ & $\stackrel{\circ}{\stackrel{0}{\circ}}$ & $\stackrel{8}{\circ}$ & $\stackrel{\infty}{\stackrel{\infty}{0}}$ & $\stackrel{0}{0}$ & $\stackrel{8}{\circ}$ & $\stackrel{t}{0}$ & $\stackrel{8}{0}$ & $\stackrel{\sigma}{0}$ & $\stackrel{8}{\circ}$ \\
\hline$\frac{\pi}{0}$ & $\frac{5}{0}$ & $\stackrel{0}{\stackrel{0}{0}}$ & $\stackrel{n}{\circ}$ & $\stackrel{ \pm}{\square}$ & $\stackrel{m}{\circ}$ & $\stackrel{\sim}{\stackrel{0}{0}}$ & $\exists$ & $\stackrel{\circ}{\stackrel{0}{0}}$ & $\stackrel{8}{\circ}$ & $\stackrel{\infty}{\stackrel{\infty}{0}}$ & $\stackrel{8}{\circ}$ & $\begin{array}{l}\qquad \\
\stackrel{0}{0} \\
0\end{array}$ & $\stackrel{m}{0}$ & $\stackrel{\Xi}{\circ}$ \\
\hline ָे & $\stackrel{9}{\circ}$ & $\stackrel{\infty}{\stackrel{\infty}{0}}$ & $\frac{1}{0}$ & $\begin{array}{l}0 \\
\stackrel{0}{0}\end{array}$ & $\frac{n}{0}$ & $\stackrel{ \pm}{\circ}$ & $\stackrel{m}{\circ}$ & $\stackrel{N}{\stackrel{0}{0}}$ & $\exists$ & $\stackrel{\circ}{\stackrel{0}{0}}$ & $\stackrel{\infty}{\stackrel{\infty}{0}}$ & : & $\stackrel{n}{\circ}$ & $\stackrel{\leftrightarrow}{0}$ \\
\hline$\stackrel{\text { }}{\tilde{o}}$ & $\tilde{\widetilde{o}}$ & $\stackrel{\text { ָे }}{0}$ & $\stackrel{\text { ঙิ }}{0}$ & $\stackrel{9}{\circ}$ & $\stackrel{\infty}{\frac{1}{0}}$ & $\frac{1}{0}$ & $\stackrel{0}{\stackrel{0}{0}}$ & $\frac{n}{0}$ & $\stackrel{ \pm}{\overrightarrow{0}}$ & $\stackrel{\sim}{\stackrel{0}{0}}$ & $\exists$ & $\begin{array}{l}\stackrel{a}{0} \\
\stackrel{0}{0}\end{array}$ & $\stackrel{0}{0}$ & $\stackrel{\leftrightarrow}{\circ}$ \\
\hline$\stackrel{n}{n}$ & 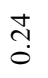 & $\stackrel{\overbrace{}}{0}$ & $\stackrel{\text { ָ̦ }}{0}$ & $\stackrel{\widetilde{N}}{\stackrel{0}{0}}$ & $\bar{\sim}$ & $\stackrel{\text { ָ̦ }}{\circ}$ & $\stackrel{9}{\circ}$ & $\stackrel{\infty}{\stackrel{0}{0}}$ & $\stackrel{0}{\stackrel{0}{0}}$ & $\stackrel{n}{\circ}$ & $\stackrel{ \pm}{\circ}$ & $\stackrel{2}{\circ}$ & $\stackrel{\circ}{\stackrel{0}{0}}$ & $\stackrel{\infty}{\stackrel{0}{0}}$ \\
\hline $\begin{array}{l}\infty \\
\stackrel{\infty}{0}\end{array}$ & $\stackrel{\overbrace{}}{o}$ & $\begin{array}{l}\text { \ִ } \\
0\end{array}$ & 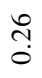 & 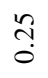 & $\stackrel{\text { I }}{\sim}$ & $\stackrel{\overbrace{}}{\stackrel{n}{n}}$ & $\stackrel{\text { ָ̦ }}{0}$ & $\stackrel{\widetilde{o}}{0}$ & $\stackrel{9}{\circ}$ & $\stackrel{\infty}{\stackrel{\infty}{0}}$ & $\frac{1}{0}$ & $\stackrel{n}{\circ}$ & $\stackrel{m}{\circ}$ & $\exists$ \\
\hline$\vec{n}$ & $\vec{m}$ & @़? & ָे & $\begin{array}{l}\infty \\
\stackrel{\infty}{0} \\
0\end{array}$ & $\hat{\sim}$ & 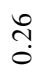 & 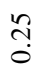 & $\stackrel{\stackrel{+}{+}}{0}$ & $\stackrel{\overbrace{}}{0}$ & 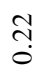 & 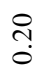 & $\stackrel{9}{\circ}$ & $\frac{1}{0}$ & $\stackrel{n}{\circ}$ \\
\hline$\stackrel{n}{m}$ & $\stackrel{+}{m}$ & $\stackrel{m}{0}$ & $\stackrel{m}{?}$ & $\stackrel{n}{0}$ & $\stackrel{m}{0}$ & ?̊ & ָे & 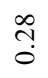 & $\stackrel{\overbrace{}}{o}$ & 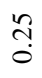 & $\stackrel{\stackrel{+}{+}}{\stackrel{0}{0}}$ & $\begin{array}{l}\text { तै } \\
\text { a }\end{array}$ & ํ. & $\stackrel{\infty}{\stackrel{0}{0}}$ \\
\hline ले & $\stackrel{\infty}{\dddot{\infty}}$ & m. & m. & $\begin{array}{l}\text { ? } \\
\text { ?. }\end{array}$ & $\stackrel{m}{0}$ & ले & $\stackrel{m}{?}$ & $\stackrel{\text { ?ै }}{3}$ & $\vec{m}$ & $\stackrel{\text { }}{0}$ & $\stackrel{\infty}{\stackrel{\infty}{0}}$ & 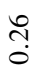 & $\stackrel{\stackrel{+}{d}}{0}$ & $\stackrel{\text { İ }}{0}$ \\
\hline$\stackrel{\infty}{\sim}$ & $\beth$ & $\mathscr{0}$ & $\cong$ & $\Xi$ & $\stackrel{m}{=}$ & $\simeq$ & $=$ & 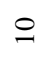 & $a$ & $\infty$ & $r$ & 0 & $n$ & 寸 \\
\hline
\end{tabular}




\begin{tabular}{|c|c|c|c|c|c|c|c|c|c|c|c|c|c|c|}
\hline$\stackrel{8}{8}$ & $\stackrel{8}{8}$ & $\stackrel{8}{8}$ & \multirow{19}{*}{ 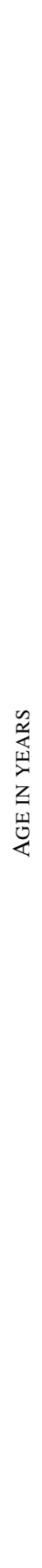 } & $\stackrel{\sim}{ \pm}$ & $\begin{array}{l}\tilde{b} \\
\dot{0}\end{array}$ & $\stackrel{n}{n}$ & $\stackrel{+}{n}$ & $\stackrel{\bar{n}}{\circ}$ & $\stackrel{g}{\stackrel{\leftrightarrow}{0}}$ & f̊f & $\begin{array}{l}0 \\
\stackrel{+}{0}\end{array}$ & $\underset{0}{\mathbb{O}}$ & 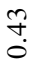 & ָ̃ \\
\hline$\stackrel{8}{\circ}$ & $\stackrel{8}{\circ}$ & $\stackrel{8}{\circ}$ & & \pm & $\begin{array}{l}\tilde{b} \\
\stackrel{0}{0}\end{array}$ & $\stackrel{\circ}{\dddot{n}}$ & $\stackrel{n}{0}$ & ?ִ & $\stackrel{\infty}{\stackrel{\infty}{0}}$ & \begin{tabular}{l}
0 \\
\multirow{0}{0}{} \\
0
\end{tabular} & $\stackrel{f}{\circ}$ & $\stackrel{?}{\stackrel{9}{0}}$ & 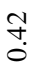 & $\underset{0}{F}$ \\
\hline $\begin{array}{l}8 \\
0 \\
0\end{array}$ & $\begin{array}{l}8 \\
0 \\
0\end{array}$ & $\begin{array}{l}8 \\
0 \\
0\end{array}$ & & $\ddot{n}$ & $\begin{array}{l}\bar{\sigma} \\
0\end{array}$ & $\begin{array}{l}\mathscr{0} \\
n \\
0\end{array}$ & ñ & \begin{tabular}{l}
$\stackrel{n}{n}$ \\
\hdashline
\end{tabular} & $\stackrel{f}{\stackrel{f}{0}}$ & \begin{tabular}{l}
0 \\
\multirow{0}{0}{} \\
0
\end{tabular} & $\stackrel{f}{\stackrel{f}{0}}$ & $\stackrel{?}{\stackrel{f}{0}}$ & $\underset{0}{F}$ & 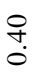 \\
\hline $\begin{array}{l}8 \\
0\end{array}$ & $\begin{array}{l}8 \\
0\end{array}$ & $\begin{array}{l}8 \\
0\end{array}$ & & 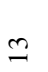 & $\begin{array}{l}\bar{\sigma} \\
0\end{array}$ & 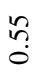 & กี & 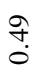 & $\stackrel{f}{f}$ & $\stackrel{\text { ra }}{\circ}$ & $\begin{array}{l}\text { J } \\
0\end{array}$ & $\stackrel{\text { I }}{0}$ & $\stackrel{F}{\tilde{0}}$ & $\begin{array}{l}\text { P. } \\
\stackrel{0}{0}\end{array}$ \\
\hline $\begin{array}{l}8 \\
0\end{array}$ & $\begin{array}{l}8 \\
0\end{array}$ & $\begin{array}{l}8 \\
0\end{array}$ & & $\stackrel{\sim}{\mathfrak{c}}$ & $\begin{array}{l}\bar{\sigma} \\
0\end{array}$ & $\begin{array}{l}n \\
n \\
0\end{array}$ & กี & 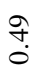 & $\stackrel{f}{f}$ & $\stackrel{\text { ra }}{\circ}$ & $\begin{array}{l}+ \\
0 \\
0\end{array}$ & $\stackrel{\text { I }}{0}$ & $\underset{0}{J}$ & $\begin{array}{l}\text { ? } \\
0\end{array}$ \\
\hline $\begin{array}{l}8 \\
0\end{array}$ & $\begin{array}{l}8 \\
0\end{array}$ & $\begin{array}{l}8 \\
0\end{array}$ & & $\cong$ & $\begin{array}{l}\bar{\sigma} \\
0\end{array}$ & $\begin{array}{l}n \\
n \\
0\end{array}$ & ก̂. & 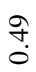 & $\stackrel{f}{f}$ & \begin{tabular}{l}
0 \\
\multirow{0}{0}{} \\
0
\end{tabular} & $\begin{array}{l}\text { J } \\
0\end{array}$ & $\stackrel{?}{\stackrel{f}{0}}$ & $\underset{0}{\overrightarrow{0}}$ & $\begin{array}{l}\text { ? } \\
0\end{array}$ \\
\hline $\begin{array}{l}8 \\
0\end{array}$ & $\begin{array}{l}8 \\
0\end{array}$ & $\begin{array}{l}8 \\
0\end{array}$ & & $\stackrel{n}{=}$ & $\begin{array}{l}\bar{\sigma} \\
0\end{array}$ & 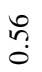 & $\stackrel{n}{n}$ & ?̊n? & $\stackrel{\infty}{\stackrel{\infty}{0}}{ }_{0}$ & \begin{tabular}{l}
0 \\
\multirow{0}{0}{} \\
0
\end{tabular} & $\begin{array}{l}\text { J } \\
0\end{array}$ & $\stackrel{?}{\stackrel{f}{0}}$ & $\stackrel{\text { I }}{0}$ & $\underset{0}{F}$ \\
\hline $\begin{array}{l}8 \\
0\end{array}$ & $\begin{array}{l}8 \\
0\end{array}$ & $\begin{array}{l}8 \\
0 \\
0\end{array}$ & & $\Xi$ & $\begin{array}{l}\tilde{\sigma} \\
0 \\
0\end{array}$ & $n$ & $\stackrel{n}{n}$ & $\bar{n}$ & 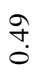 & f̊ & $\stackrel{n}{\stackrel{n}{0}}$ & $\underset{\stackrel{+}{0}}{\stackrel{f}{0}}$ & $\stackrel{?}{\stackrel{9}{0}}$ & F. \\
\hline $\begin{array}{l}8 \\
0\end{array}$ & $\begin{array}{l}8 \\
0\end{array}$ & $\begin{array}{l}8 \\
0 \\
0\end{array}$ & & $\stackrel{n}{\varrho}$ & $\begin{array}{l}\text { 乃़} \\
0\end{array}$ & $\begin{array}{l}\infty \\
n \\
0\end{array}$ & $\stackrel{+}{n}$ & กี & $\begin{array}{l}\stackrel{n}{n} \\
0\end{array}$ & 市 & $\begin{array}{l}0 \\
\stackrel{0}{0}\end{array}$ & $\stackrel{?}{\stackrel{f}{0}}$ & $\stackrel{f}{+}$ & $\stackrel{T}{\stackrel{5}{0}}$ \\
\hline $\begin{array}{l}8 \\
0\end{array}$ & $\begin{array}{l}8 \\
0\end{array}$ & $\begin{array}{l}8 \\
0\end{array}$ & & $\varrho$ & $\begin{array}{l}n \\
\mathfrak{0} \\
0\end{array}$ & ஜ̂? & $\begin{array}{l}0 \\
n \\
0\end{array}$ & $\stackrel{m}{n}$ & $\tilde{n}$ & ț. & $\stackrel{\substack{+0}}{0}$ & $\stackrel{0}{+}$ & $\stackrel{n}{\stackrel{n}{0}}$ & t. \\
\hline $\begin{array}{l}8 \\
0\end{array}$ & $\begin{array}{l}8 \\
0\end{array}$ & $\begin{array}{l}8 \\
0\end{array}$ & & $\tilde{a}$ & $\begin{array}{l}\stackrel{0}{0} \\
0\end{array}$ & $\begin{array}{l}\bar{\sigma} \\
0\end{array}$ & 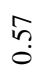 & ñ & $\stackrel{n}{n}$ & $\tilde{n}$ & 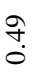 & 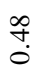 & $\stackrel{f}{f}$ & $\stackrel{n}{\stackrel{n}{0}}$ \\
\hline $\begin{array}{l}8 \\
0\end{array}$ & $\begin{array}{l}8 \\
0\end{array}$ & $\begin{array}{l}8 \\
0\end{array}$ & & $a$ & $\begin{array}{l}\infty \\
\stackrel{\infty}{0} \\
0\end{array}$ & $\begin{array}{l}\tilde{6} \\
0 \\
0\end{array}$ & $\stackrel{n}{n}$ & ñ & ñn & $\tilde{n}$ & $\bar{n}$ & ?̊n & 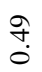 & f̊ \\
\hline $\begin{array}{l}8 \\
0\end{array}$ & $\begin{array}{l}8 \\
0\end{array}$ & $\begin{array}{l}8 \\
0 \\
0\end{array}$ & & $\begin{array}{l}n \\
\infty \\
\infty\end{array}$ & $\vec{i}$ & $\begin{array}{l}n \\
0 \\
0\end{array}$ & $\begin{array}{l}\tilde{\sigma} \\
\dot{0}\end{array}$ & ñ? & $\tilde{n}$ & ñ & ra & กี & $\bar{n}$ & ?̊ \\
\hline $\begin{array}{l}\text { Oे } \\
0\end{array}$ & $\begin{array}{l}8 \\
0\end{array}$ & $\begin{array}{l}8 \\
0 \\
0\end{array}$ & & $\infty$ & $\stackrel{m}{\mathfrak{0}}$ & $\begin{array}{l}\infty \\
0 \\
0 \\
0\end{array}$ & $\begin{array}{l}\mathbb{J} \\
\dot{0}\end{array}$ & $\begin{array}{l}\text { Vै } \\
\stackrel{0}{0}\end{array}$ & ñ? & $\begin{array}{l}\infty \\
n \\
n\end{array}$ & "ron & ñ & ñ? & ñ? \\
\hline $\begin{array}{l}0 \\
0 \\
0\end{array}$ & $\begin{array}{l}\text { Oे } \\
0\end{array}$ & $\begin{array}{l}8 \\
0 \\
0\end{array}$ & & $\stackrel{n}{r}$ & $\stackrel{0}{\stackrel{0}{0}}$ & $\stackrel{?}{\stackrel{0}{0}}$ & $\begin{array}{l}\tilde{\sigma} \\
0\end{array}$ & $\begin{array}{l}\text { ț } \\
\stackrel{0}{0}\end{array}$ & $\begin{array}{l}\tilde{\sigma} \\
0\end{array}$ & : & ñ? & $\stackrel{\infty}{n}$ & $\begin{array}{l}0 \\
\text { n? } \\
0\end{array}$ & $n$ \\
\hline$\stackrel{\circ}{\circ}$ & $\stackrel{n}{\circ}$ & $\stackrel{8}{\circ}$ & & $r$ & 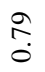 & $\underset{⿱}{\stackrel{0}{0}}$ & $\stackrel{?}{\stackrel{0}{0}}$ & $\begin{array}{l}\infty \\
\stackrel{0}{0} \\
0\end{array}$ & $\begin{array}{l}n \\
0 \\
0\end{array}$ & $\begin{array}{l}\text { t. } \\
\text {. }\end{array}$ & $\begin{array}{l}\mathcal{O} \\
\stackrel{0}{0}\end{array}$ & $\stackrel{\square}{0}$ & nิ? & $\begin{array}{l}\infty \\
? \\
0\end{array}$ \\
\hline$\frac{\mathfrak{T}}{\stackrel{0}{0}}$ & $\begin{array}{l}\stackrel{\theta}{\partial} \\
\dot{0}\end{array}$ & $\stackrel{\leftrightarrow}{0}$ & & $\ddot{n}$ & $\begin{array}{l}\text { Oै } \\
\stackrel{0}{0}\end{array}$ & $\stackrel{\hat{z}}{0}$ & $\stackrel{+}{\stackrel{t}{0}}$ & $\vec{r}$ & $\stackrel{a}{\mathfrak{b}}$ & గ్రి: & $\begin{array}{l}n \\
\stackrel{0}{0} \\
\end{array}$ & $\begin{array}{l}\text { ț } \\
\stackrel{0}{0}\end{array}$ & 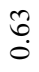 & $\begin{array}{l}\mathcal{S} \\
\stackrel{0}{0}\end{array}$ \\
\hline$\stackrel{0}{\frac{0}{0}}$ & $\frac{N}{0}$ & $\stackrel{0}{0}$ & & 6 & $\begin{array}{l}\stackrel{0}{\infty} \\
\stackrel{0}{0}\end{array}$ & $\begin{array}{l}\bar{\infty} \\
0\end{array}$ & $\stackrel{f}{0}$ & $\stackrel{n}{\stackrel{2}{0}}$ & $\stackrel{1}{0}$ & $\stackrel{ }{\circ}$ & $\begin{array}{l}\hat{0} \\
\stackrel{0}{0}\end{array}$ & $\begin{array}{l}\infty \\
\stackrel{0}{0} \\
0\end{array}$ & $\begin{array}{l}: \\
: \\
:\end{array}$ & $\begin{array}{l}n \\
\stackrel{0}{0} \\
0\end{array}$ \\
\hline$\stackrel{\text { ঙ̣ }}{0}$ & $\stackrel{0}{\stackrel{0}{0}}$ & $\vec{\square}$ & & $\stackrel{n}{i n}$ & $\stackrel{̊}{\circ}$ & $\begin{array}{l}\mathscr{\infty} \\
\infty \\
0\end{array}$ & $\stackrel{\infty}{\infty}$ & $\stackrel{\overbrace{}}{\circ}$ & $\stackrel{\circ}{\stackrel{0}{0}}$ & $\stackrel{n}{\stackrel{n}{0}}$ & $\stackrel{m}{\stackrel{0}{\circ}}$ & $\stackrel{\mathbb{N}}{\stackrel{0}{0}}$ & $\stackrel{?}{\stackrel{0}{\circ}}$ & : \\
\hline$m$ & $N$ & - & 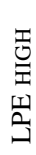 & 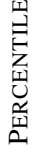 & ลे & $\infty$ & $\hat{a}$ & ฉ̊ & $\approx$ & d & $\alpha$ & $\sigma$ & $\bar{a}$ & ฉ \\
\hline
\end{tabular}




\begin{tabular}{|c|c|c|c|c|c|c|c|c|c|c|c|c|c|c|}
\hline$\underset{0}{\exists}$ & $\begin{array}{l}\text { + } \\
\stackrel{0}{0}\end{array}$ & ભ़े & $\stackrel{\infty}{\tilde{m}}$ & $\stackrel{n}{0}$ & 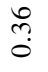 & $\stackrel{n}{m}$ & $\stackrel{+}{\text { m. }}$ & $\stackrel{+}{\text { m. }}$ & $\stackrel{m}{0}$ & ?ొ & $\begin{array}{l}0 \\
\stackrel{1}{0}\end{array}$ & $\stackrel{\text { Oִ }}{0}$ & $\stackrel{8}{8}$ & $\stackrel{8}{8}$ \\
\hline $\begin{array}{l}\stackrel{+}{+} \\
\dot{0}\end{array}$ & ڤे & 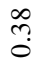 & $\stackrel{n}{0}$ & ? & $\stackrel{n}{\tilde{m}}$ & $\stackrel{+}{\text { m}}$ & $\stackrel{+}{m}$ & $\stackrel{m}{\oplus}$ & $\stackrel{\text { ?ै }}{0}$ & $\stackrel{\overbrace ָ}{\grave{0}}$ & $\frac{n}{0}$ & $\begin{array}{l}\mathrm{O} \\
\stackrel{0}{0}\end{array}$ & $\stackrel{8}{:}$ & $\begin{array}{l}\stackrel{8}{0} \\
\stackrel{0}{0}\end{array}$ \\
\hline ڤి. & 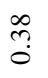 & $\stackrel{n}{0}$ & 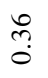 & $\stackrel{n}{m}$ & $\stackrel{+}{m}$ & $\stackrel{+}{m}$ & $\stackrel{m}{0}$ & $\stackrel{\sim}{\tilde{o}}$ & $\overrightarrow{0}$ & 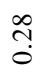 & $\stackrel{n}{\circ}$ & $\ddot{0}$ & $\stackrel{8}{\circ}$ & $\stackrel{8}{\circ}$ \\
\hline ભે & 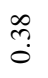 & $\stackrel{m}{0}$ & 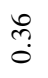 & $\stackrel{n}{m}$ & $\stackrel{+}{m}$ & $\stackrel{m}{?}$ & $\stackrel{m}{0}$ & $\stackrel{\sim}{0}$ & $\vec{m}$ & 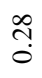 & $\stackrel{ \pm}{\oplus}$ & $\stackrel{\Xi}{0}$ & $\stackrel{8}{\circ}$ & $\stackrel{8}{\circ}$ \\
\hline ભे & $\stackrel{\infty}{\prod_{0}^{\infty}}$ & $\stackrel{n}{0}$ & @ొ & $\stackrel{n}{m}$ & $\stackrel{+}{m}$ & $\stackrel{m}{?}$ & $\stackrel{\sim}{\tilde{0}}$ & $\stackrel{\sim}{\tilde{0}}$ & $\vec{m}$ & $\stackrel{\infty}{\stackrel{\infty}{0}}$ & $\stackrel{ \pm}{\circ}$ & $\stackrel{\Xi}{0}$ & $\stackrel{8}{\circ}$ & $\stackrel{8}{\circ}$ \\
\hline ભे? & $\stackrel{\infty}{\prod_{0}^{\infty}}$ & $\stackrel{m}{0}$ & 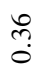 & $\stackrel{n}{m}$ & $\stackrel{+}{m}$ & $\stackrel{m}{?}$ & $\stackrel{m}{0}$ & $\stackrel{\sim}{\tilde{0}}$ & $\vec{m}$ & $\stackrel{\infty}{\stackrel{\infty}{0}}$ & $\stackrel{ \pm}{\dot{0}}$ & $\stackrel{\Xi}{0}$ & $\stackrel{8}{\circ}$ & $\stackrel{8}{\circ}$ \\
\hline ભे? & $\stackrel{\infty}{\overbrace{0}^{\infty}}$ & $\stackrel{m}{0}$ & n़? & 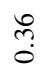 & $\stackrel{n}{n}$ & $\stackrel{+}{m}$ & $\stackrel{m}{0}$ & $\stackrel{\sim}{\tilde{0}}$ & $\stackrel{\sim}{0}$ & $\stackrel{\infty}{\stackrel{\infty}{0}}$ & $\stackrel{n}{\circ}$ & $\stackrel{\Xi}{0}$ & $\stackrel{8}{\circ}$ & $\stackrel{8}{\circ}$ \\
\hline$\stackrel{+}{+}$ & ڤે & $\stackrel{\infty}{\stackrel{\infty}{0}}$ & $\hat{n}$ & ?! & 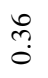 & $\stackrel{n}{m}$ & $\stackrel{m}{m}$ & $\stackrel{m}{0}$ & $\stackrel{m}{0}$ & $\stackrel{\text { సे }}{0}$ & $\begin{array}{l}\stackrel{0}{0} \\
0\end{array}$ & 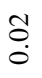 & $\stackrel{8}{\circ}$ & $\stackrel{8}{\circ}$ \\
\hline$\underset{0}{\exists}$ & $\stackrel{?}{+}$ & ڤे? & $\stackrel{\infty}{\stackrel{\infty}{0}}$ & $\stackrel{\infty}{\stackrel{\infty}{0}}$ & $\tilde{m}$ & 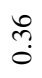 & $\stackrel{m}{0}$ & $\stackrel{+}{\tilde{O}}$ & $\stackrel{+}{0}$ & @ి & $\frac{1}{0}$ & $\stackrel{\overbrace{}}{0}$ & $\stackrel{8}{\circ}$ & $\stackrel{8}{\circ}$ \\
\hline$\stackrel{?}{\leftrightarrow}$ & $\stackrel{\stackrel{f}{\leftrightarrow}}{\circ}$ & $\stackrel{f}{\circ}$ & $\stackrel{?}{+}$ & ڤે? & 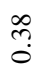 & $\stackrel{n}{0}$ & $\stackrel{n}{0}$ & 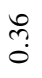 & $\stackrel{n}{0}$ & $\stackrel{n}{0}$ & $\stackrel{\infty}{\stackrel{0}{0}}$ & $\stackrel{n}{\circ}$ & $\ddot{0}$ & $\stackrel{\square}{\circ}$ \\
\hline$\stackrel{+}{\stackrel{+}{0}}$ & $\stackrel{?}{\stackrel{0}{0}}$ & $\stackrel{\text { I }}{\circ}$ & $\underset{f}{\vec{\sigma}}$ & $\underset{\ominus}{\ni}$ & 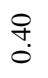 & ભి & $\stackrel{\infty}{\prod_{0}^{\infty}}$ & $\stackrel{n}{0}$ & $\stackrel{n}{0}$ & $\stackrel{m}{0}$ & 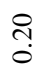 & $\stackrel{\circ}{\circ}$ & $\stackrel{\mathscr{O}}{0}$ & 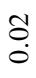 \\
\hline$\stackrel{+}{+}$ & $\stackrel{f}{\stackrel{n}{0}}$ & $\stackrel{⿱}{*}$ & $\stackrel{?}{\stackrel{0}{0}}$ & $\stackrel{\mathscr{P}}{\circ}$ & $\stackrel{\stackrel{f}{\leftrightarrow}}{\circ}$ & $\stackrel{f}{\circ}$ & 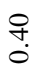 & ले & ले & $\stackrel{n}{m}$ & $\stackrel{\widetilde{N}}{\tilde{o}}$ & $\stackrel{\infty}{\circ}$ & $\stackrel{n}{0}$ & $\begin{array}{l} \pm \\
\stackrel{0}{0}\end{array}$ \\
\hline$\stackrel{\leftrightarrow}{\stackrel{q}{0}}$ & $\stackrel{\infty}{\stackrel{\infty}{0}}$ & $\stackrel{\leftrightarrow}{\stackrel{f}{0}}$ & $\begin{array}{l}0 \\
\stackrel{+}{0}\end{array}$ & $\stackrel{n}{\stackrel{n}{0}}$ & $\stackrel{+}{+}$ & $\stackrel{?}{\leftrightarrow}$ & $\stackrel{\sim}{\stackrel{f}{0}}$ & $\stackrel{\sim}{\stackrel{f}{0}}$ & $\underset{\dot{J}}{\vec{j}}$ & 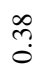 & 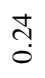 & $=$ & $\stackrel{i}{0}$ & $\stackrel{\circ}{\stackrel{\circ}{0}}$ \\
\hline$\tilde{n}$ & $\stackrel{n}{n}$ & $\stackrel{\stackrel{q}{+}}{\circ}$ & $\stackrel{\infty}{\stackrel{\infty}{0}}$ & $\stackrel{\leftrightarrow}{\circ}$ & $\begin{array}{l}\stackrel{+}{+} \\
\stackrel{0}{0}\end{array}$ & $\stackrel{\circ}{\stackrel{0}{0}}$ & $\stackrel{\leftrightarrow}{\stackrel{0}{0}}$ & $\stackrel{f}{\stackrel{f}{0}}$ & $\stackrel{?}{\stackrel{0}{0}}$ & $\stackrel{+}{\stackrel{q}{0}}$ & 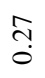 & $\stackrel{m}{\circ}$ & $\stackrel{\circ}{\circ}$ & $\stackrel{\circ}{\circ}$ \\
\hline$\stackrel{+}{n}$ & $\stackrel{n}{n}$ & $\stackrel{n}{n}$ & $\ddot{n}$ & ?̊? & $\stackrel{\stackrel{q}{0}}{\circ}$ & $\stackrel{\infty}{+}$ & $\stackrel{\infty}{\stackrel{\infty}{0}}$ & $\stackrel{\text { fo }}{\circ}$ & $\stackrel{+}{\stackrel{0}{0}}$ & $\stackrel{?}{\circ}$ & సे & $\stackrel{0}{\stackrel{0}{0}}$ & $\stackrel{m}{\circ}$ & $\stackrel{\sim}{\stackrel{0}{0}}$ \\
\hline$\tilde{n}$ & $\stackrel{?}{n}$ & $\tilde{n}$ & $\stackrel{+}{n}$ & $\stackrel{n}{n}$ & $\stackrel{n}{n}$ & กี & $\bar{n}$ & $\stackrel{n}{n}$ & $\stackrel{\stackrel{\leftrightarrow}{\circ}}{\circ}$ & $\stackrel{\circ}{\stackrel{0}{0}}$ & $\stackrel{m}{0}$ & $\stackrel{9}{\circ}$ & $\stackrel{0}{\stackrel{0}{0}}$ & $\stackrel{n}{\circ}$ \\
\hline $\begin{array}{l}\stackrel{8}{0} \\
\dot{0}\end{array}$ & $\stackrel{n}{0}$ & $\begin{array}{l}\infty \\
n \\
0\end{array}$ & $\stackrel{\infty}{n}$ & $\tilde{n}$ & $\begin{array}{l}\stackrel{n}{0} \\
\stackrel{n}{0}\end{array}$ & $\stackrel{n}{n}$ & $\stackrel{+}{n}$ & $\stackrel{n}{n}$ & $\stackrel{n}{0}$ & $\stackrel{\stackrel{q}{\circ}}{\circ}$ & 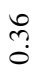 & $\stackrel{\text { İ }}{0}$ & $\stackrel{9}{\circ}$ & $\stackrel{\infty}{\stackrel{0}{0}}$ \\
\hline $\begin{array}{l}\text { ț } \\
\stackrel{0}{0}\end{array}$ & $\begin{array}{l}\Re \\
\stackrel{0}{0}\end{array}$ & $\begin{array}{l}\text { Ț } \\
\stackrel{0}{0}\end{array}$ & $\begin{array}{l}\vec{b} \\
\dot{0}\end{array}$ & $\begin{array}{l}\stackrel{8}{0} \\
\dot{0}\end{array}$ & $\stackrel{n}{0}$ & ñ. & $\stackrel{\infty}{n}$ & $\stackrel{n}{n}$ & $\begin{array}{l}n \\
\stackrel{n}{0}\end{array}$ & $\stackrel{n}{n}$ & 定 & $\begin{array}{l}\stackrel{\circ}{0} \\
\stackrel{0}{0}\end{array}$ & $\stackrel{\overbrace{}}{0}$ & $\stackrel{\text { İ }}{0}$ \\
\hline $\begin{array}{l}\infty \\
\stackrel{0}{0} \\
\dot{0}\end{array}$ & $\begin{array}{l}\hat{\sigma} \\
\dot{0}\end{array}$ & $\begin{array}{l}: \\
\stackrel{0}{0}\end{array}$ & $\begin{array}{l}\because \\
\ddot{0}\end{array}$ & $\begin{array}{l}\vec{t} \\
\stackrel{0}{0}\end{array}$ & $\begin{array}{l}\hat{b} \\
\dot{0}\end{array}$ & $\begin{array}{l}\text { Ob. } \\
\stackrel{0}{0}\end{array}$ & $\begin{array}{l}\text { Cె } \\
\stackrel{0}{0}\end{array}$ & $\begin{array}{l}\bar{\sigma} \\
\stackrel{0}{0}\end{array}$ & $\begin{array}{l}\stackrel{8}{0} \\
\stackrel{0}{0}\end{array}$ & $\stackrel{n}{n}$ & $\underset{:}{\stackrel{f}{0}}$ & ़ֻ & 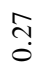 & 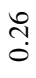 \\
\hline ळ) & $\stackrel{\infty}{\infty}$ & $\hat{\infty}$ & $\infty$ & 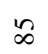 & 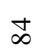 & $\infty$ & $\widetilde{\infty}$ & $\bar{\infty}$ & $\stackrel{\infty}{\infty}$ & $\stackrel{2}{\sim}$ & in & $\stackrel{2}{\sim}$ & ㄱ. & 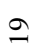 \\
\hline
\end{tabular}




\begin{tabular}{|c|c|c|c|c|c|c|c|c|c|c|c|c|c|c|}
\hline$\underset{0}{8}$ & $\stackrel{8}{8}$ & $\stackrel{8}{8}$ & $\stackrel{8}{\circ}$ & $\stackrel{8}{8}$ & : & $\begin{array}{l}8 \\
\stackrel{0}{0} \\
\end{array}$ & $\stackrel{8}{\circ}$ & $\stackrel{8}{8}$ & $\stackrel{8}{8}$ & $\begin{array}{l}8 \\
\stackrel{0}{0} \\
\end{array}$ & $\stackrel{8}{8}$ & $\begin{array}{l}8 \\
\stackrel{0}{0}\end{array}$ & $\underset{0}{8}$ & $\underset{8}{8}$ \\
\hline $\begin{array}{l}\stackrel{0}{0} \\
\stackrel{0}{ }\end{array}$ & $\stackrel{8}{\circ}$ & $\stackrel{8}{\circ}$ & $\stackrel{8}{\circ}$ & $\stackrel{8}{\circ}$ & $\stackrel{8}{\circ}$ & $\begin{array}{l}8 \\
\stackrel{0}{0}\end{array}$ & $\stackrel{8}{\circ}$ & $\stackrel{8}{\circ}$ & $\stackrel{\circ}{\circ}$ & $\begin{array}{l}8 \\
\stackrel{0}{0}\end{array}$ & $\stackrel{\circ}{\circ}$ & $\begin{array}{l}\stackrel{8}{0} \\
\stackrel{0}{0}\end{array}$ & $\begin{array}{l}8 \\
\stackrel{0}{0}\end{array}$ & $\begin{array}{l}\stackrel{8}{0} \\
\dot{0}\end{array}$ \\
\hline $\begin{array}{l}8 \\
0\end{array}$ & $\begin{array}{l}8 \\
0 \\
0\end{array}$ & $\begin{array}{l}8 \\
0 \\
0\end{array}$ & $\stackrel{8}{\circ}$ & $\begin{array}{l}8 \\
0\end{array}$ & $\begin{array}{l}\stackrel{0}{0} \\
\stackrel{0}{1}\end{array}$ & $\begin{array}{l}8 \\
\stackrel{0}{0}\end{array}$ & $\stackrel{8}{\circ}$ & $\begin{array}{l}8 \\
0 \\
0\end{array}$ & $\begin{array}{l}8 \\
0\end{array}$ & $\stackrel{8}{8}$ & $\begin{array}{l}8 \\
0\end{array}$ & $\begin{array}{l}\stackrel{8}{0} \\
0\end{array}$ & $\begin{array}{l}\stackrel{8}{0} \\
0\end{array}$ & $\begin{array}{l}8 \\
0 \\
0\end{array}$ \\
\hline$\stackrel{8}{\circ}$ & $\stackrel{8}{\circ}$ & $\stackrel{8}{8}$ & $\stackrel{8}{\circ}$ & $\stackrel{8}{8}$ & $\stackrel{8}{\circ}$ & $\begin{array}{l}8 \\
\\
0\end{array}$ & $\stackrel{8}{\circ}$ & $\stackrel{8}{8}$ & $\stackrel{8}{\circ}$ & \begin{tabular}{l}
8 \\
\hdashline
\end{tabular} & $\stackrel{8}{8}$ & $\begin{array}{l}8 \\
\stackrel{0}{0}\end{array}$ & \begin{tabular}{l}
8 \\
\hdashline
\end{tabular} & $\stackrel{8}{\circ}$ \\
\hline$\stackrel{8}{\circ}$ & $\stackrel{8}{\circ}$ & $\stackrel{8}{\circ}$ & $\stackrel{8}{\circ}$ & $\stackrel{8}{8}$ & $\stackrel{8}{\circ}$ & $\begin{array}{l}8 \\
0 \\
0\end{array}$ & $\stackrel{8}{\circ}$ & $\stackrel{8}{8}$ & $\stackrel{8}{\circ}$ & $\begin{array}{l}8 \\
\stackrel{0}{0}\end{array}$ & $\stackrel{8}{8}$ & $\begin{array}{l}8 \\
\stackrel{0}{0}\end{array}$ & \begin{tabular}{l}
8 \\
\hdashline
\end{tabular} & $\stackrel{8}{8}$ \\
\hline$\stackrel{8}{8}$ & $\begin{array}{l}8 \\
0\end{array}$ & $\begin{array}{l}8 \\
0\end{array}$ & $\stackrel{8}{\circ}$ & $\begin{array}{l}8 \\
0\end{array}$ & $\stackrel{8}{0}$ & $\begin{array}{l}8 \\
0\end{array}$ & $\stackrel{8}{0}$ & $\begin{array}{l}8 \\
0\end{array}$ & $\begin{array}{l}8 \\
0\end{array}$ & $\stackrel{8}{\circ}$ & $\underset{0}{8}$ & $\begin{array}{l}8 \\
0\end{array}$ & $\stackrel{8}{8}$ & $\begin{array}{l}8 \\
0 \\
0\end{array}$ \\
\hline$\stackrel{8}{\circ}$ & $\stackrel{8}{\circ}$ & $\stackrel{8}{\circ}$ & $\stackrel{8}{\circ}$ & $\stackrel{8}{8}$ & $\stackrel{8}{\circ}$ & $\begin{array}{l}8 \\
0\end{array}$ & $\stackrel{8}{\circ}$ & $\stackrel{8}{\circ}$ & $\stackrel{8}{\circ}$ & \begin{tabular}{l}
8 \\
\hdashline
\end{tabular} & $\stackrel{8}{\circ}$ & $\begin{array}{l}8 \\
\stackrel{0}{0}\end{array}$ & $\stackrel{8}{\circ}$ & $\stackrel{8}{8}$ \\
\hline $\begin{array}{l}8 \\
0\end{array}$ & $\begin{array}{l}8 \\
0\end{array}$ & $\begin{array}{l}8 \\
0 \\
0\end{array}$ & $\stackrel{8}{\circ}$ & $\begin{array}{l}8 \\
0\end{array}$ & $\stackrel{8}{\circ}$ & $\begin{array}{l}8 \\
:\end{array}$ & $\stackrel{8}{\circ}$ & $\begin{array}{l}8 \\
0 \\
0\end{array}$ & $\begin{array}{l}8 \\
0\end{array}$ & $\stackrel{8}{\circ}$ & $\begin{array}{l}8 \\
0\end{array}$ & $\begin{array}{l}\stackrel{8}{0} \\
0\end{array}$ & $\begin{array}{l}8 \\
0\end{array}$ & $\begin{array}{l}8 \\
0 \\
0\end{array}$ \\
\hline $\begin{array}{l}8 \\
0\end{array}$ & $\stackrel{8}{8}$ & $\stackrel{8}{8}$ & $\stackrel{8}{\circ}$ & $\stackrel{8}{8}$ & $\stackrel{8}{8}$ & $\begin{array}{l}8 \\
0 \\
0\end{array}$ & $\stackrel{8}{\circ}$ & $\stackrel{8}{8}$ & $\stackrel{8}{8}$ & $\stackrel{8}{8}$ & $\stackrel{8}{\circ}$ & \begin{tabular}{l}
8 \\
\hdashline
\end{tabular} & $\stackrel{8}{8}$ & $\stackrel{8}{8}$ \\
\hline$\stackrel{8}{\circ}$ & $\stackrel{8}{\circ}$ & $\stackrel{8}{\circ}$ & $\stackrel{8}{\circ}$ & $\stackrel{8}{\circ}$ & $\stackrel{8}{\circ}$ & $\begin{array}{l}8 \\
\stackrel{0}{0}\end{array}$ & $\stackrel{8}{\circ}$ & $\stackrel{8}{\circ}$ & $\stackrel{8}{\circ}$ & $\begin{array}{l}8 \\
\stackrel{0}{0}\end{array}$ & $\stackrel{8}{\circ}$ & $\begin{array}{l}8 \\
\stackrel{0}{0}\end{array}$ & $\stackrel{8}{\circ}$ & $\stackrel{8}{8}$ \\
\hline $\begin{array}{l}\text { ô } \\
\stackrel{0}{0}\end{array}$ & $\stackrel{\Xi}{0}$ & $\stackrel{8}{8}$ & $\stackrel{8}{\circ}$ & $\stackrel{8}{8}$ & $\stackrel{8}{8}$ & $\begin{array}{l}8 \\
\\
0\end{array}$ & $\stackrel{8}{\circ}$ & $\stackrel{8}{8}$ & $\stackrel{8}{\circ}$ & $\stackrel{8}{\circ}$ & $\stackrel{8}{8}$ & $\begin{array}{l}8 \\
\stackrel{0}{0}\end{array}$ & $\stackrel{8}{\circ}$ & $\stackrel{8}{\circ}$ \\
\hline $\begin{array}{l} \pm \\
0 \\
0\end{array}$ & $\begin{array}{l}0 \\
0 \\
0\end{array}$ & $\begin{array}{l}\text { Oे } \\
0\end{array}$ & $\ddot{0}$ & $\begin{array}{l}8 \\
0\end{array}$ & $\begin{array}{l}8 \\
0\end{array}$ & $\stackrel{8}{8}$ & $\stackrel{8}{\circ}$ & $\begin{array}{l}8 \\
0\end{array}$ & $\begin{array}{l}8 \\
0\end{array}$ & $\stackrel{8}{0}$ & $\stackrel{8}{8}$ & $\begin{array}{l}8 \\
0\end{array}$ & $\stackrel{8}{8}$ & $\begin{array}{l}8 \\
0 \\
0\end{array}$ \\
\hline $\begin{array}{l}\stackrel{0}{0} \\
0\end{array}$ & $\begin{array}{l}n \\
0 \\
0\end{array}$ & $\begin{array}{l}\dot{O} \\
\dot{0}\end{array}$ & $\stackrel{\overbrace{}}{0}$ & $\begin{array}{l}\text { ô. } \\
0 \\
0\end{array}$ & $\begin{array}{l}\text { ô. } \\
0 \\
0\end{array}$ & $\begin{array}{l}0 \\
0 \\
0\end{array}$ & $\stackrel{8}{\circ}$ & $\begin{array}{l}8 \\
0 \\
0\end{array}$ & $\begin{array}{l}8 \\
0\end{array}$ & $\stackrel{8}{\circ}$ & $\begin{array}{l}8 \\
0\end{array}$ & $\begin{array}{l}8 \\
0\end{array}$ & $\begin{array}{l}\stackrel{8}{0} \\
\stackrel{0}{0}\end{array}$ & $\begin{array}{l}8 \\
0 \\
0\end{array}$ \\
\hline $\begin{array}{l}\infty \\
\stackrel{0}{0} \\
0\end{array}$ & $\begin{array}{l}\infty \\
\stackrel{0}{0}\end{array}$ & $\begin{array}{l}\hat{0} \\
0\end{array}$ & $\stackrel{\circ}{\circ}$ & $\begin{array}{l}n \\
0 \\
0\end{array}$ & $\begin{array}{l} \pm \\
0 \\
0\end{array}$ & $\begin{array}{l}\hat{o} \\
0 \\
0\end{array}$ & $\stackrel{\sigma}{0}$ & $\stackrel{0}{0}$ & $\begin{array}{l}8 \\
0\end{array}$ & $\stackrel{8}{\circ}$ & $\begin{array}{l}8 \\
0\end{array}$ & $\begin{array}{l}\stackrel{8}{0} \\
0\end{array}$ & $\begin{array}{l}8 \\
0\end{array}$ & $\begin{array}{l}8 \\
0 \\
0\end{array}$ \\
\hline $\overrightarrow{0}$ & $\stackrel{0}{\stackrel{0}{0}}$ & $\stackrel{0}{\stackrel{0}{0}}$ & $\stackrel{\circ}{\circ}$ & $\begin{array}{l}\infty \\
\stackrel{0}{0}\end{array}$ & $\hat{0}$ & $\begin{array}{l}\stackrel{8}{0} \\
\stackrel{0}{0}\end{array}$ & $\stackrel{?}{\circ}$ & $\begin{array}{l}\text { Jे } \\
0\end{array}$ & 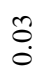 & 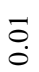 & $\stackrel{8}{:}$ & $\stackrel{8}{\stackrel{0}{0}}$ & $\stackrel{8}{\circ}$ & $\begin{array}{l}8 \\
0 \\
0\end{array}$ \\
\hline$\underset{0}{ \pm}$ & $\stackrel{m}{\overrightarrow{0}}$ & $\stackrel{m}{0}$ & $\stackrel{\frac{1}{0}}{0}$ & $\overrightarrow{0}$ & $\stackrel{0}{\stackrel{0}{0}}$ & oे. & $\stackrel{\infty}{\circ}$ & $\hat{0}$ & $\begin{array}{l}\stackrel{0}{0} \\
\stackrel{0}{0}\end{array}$ & $\begin{array}{l}\text { d. } \\
\stackrel{0}{0}\end{array}$ & 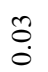 & $\ddot{0}$ & $\begin{array}{l}8 \\
0\end{array}$ & $\stackrel{8}{0}$ \\
\hline$\stackrel{\infty}{0}$ & $\frac{1}{0}$ & $\frac{0}{0}$ & $\frac{n}{0}$ & $\underset{0}{ \pm}$ & $\stackrel{m}{0}$ & $\frac{N}{0}$ & $=$ & $\stackrel{0}{\stackrel{0}{0}}$ & $\stackrel{\circ}{0}$ & $\begin{array}{l}\infty \\
\stackrel{0}{0}\end{array}$ & $\stackrel{0}{\circ}$ & $\stackrel{n}{0}$ & $\stackrel{n}{0}$ & $\ddot{0}$ \\
\hline$\tilde{\widetilde{\jmath}}$ & $\overrightarrow{\tilde{o}}$ & तి & $\stackrel{2}{0}$ & $\stackrel{\infty}{\overrightarrow{0}}$ & $\frac{1}{0}$ & $\stackrel{0}{\stackrel{0}{0}}$ & $\frac{n}{0}$ & $\stackrel{ \pm}{0}$ & $\stackrel{m}{\overrightarrow{0}}$ & $\overrightarrow{0}$ & $\stackrel{0}{\stackrel{0}{0}}$ & $\begin{array}{l}\infty \\
\stackrel{0}{0} \\
0\end{array}$ & $\stackrel{0}{0}$ & $\begin{array}{l}n \\
0 \\
0\end{array}$ \\
\hline$\stackrel{n}{n}$ & 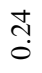 & 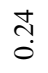 & $\stackrel{\overbrace{}}{0}$ & 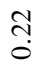 & $\stackrel{\vec{N}}{0}$ & $\begin{array}{l}\text { ָे } \\
\text {. }\end{array}$ & $\stackrel{2}{\circ}$ & $\frac{\infty}{\stackrel{0}{0}}$ & $\frac{1}{0}$ & $\frac{n}{0}$ & $\stackrel{ \pm}{\overrightarrow{0}}$ & $\frac{1}{0}$ & $\overrightarrow{0}$ & $\begin{array}{l}\stackrel{\theta}{0} \\
\dot{0}\end{array}$ \\
\hline$\stackrel{\infty}{\sim}$ & I & $\stackrel{0}{-}$ & $\stackrel{n}{n}$ & \pm & 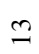 & $\simeq$ & $=$ & $\varrho$ & $a$ & $\infty$ & $r$ & 6 & $n$ & $\checkmark$ \\
\hline
\end{tabular}




\begin{tabular}{|c|c|c|}
\hline$\stackrel{8}{0}$ & $\stackrel{8}{0}$ & $\stackrel{8}{0}$ \\
\hline$\stackrel{8}{\circ}$ & $\stackrel{8}{\circ}$ & $\stackrel{8}{\circ}$ \\
\hline$\stackrel{8}{\circ}$ & $\stackrel{8}{\circ}$ & $\stackrel{8}{0}$ \\
\hline 8 & $\stackrel{8}{\circ}$ & $\stackrel{8}{0}$ \\
\hline$\stackrel{8}{\circ}$ & $\stackrel{8}{\circ}$ & $\stackrel{8}{\circ}$ \\
\hline$\stackrel{8}{\circ}$ & $\stackrel{8}{\circ}$ & $\stackrel{8}{\circ}$ \\
\hline$\stackrel{8}{0}$ & $\stackrel{8}{0}$ & $\stackrel{8}{0}$ \\
\hline$\stackrel{8}{\circ}$ & $\stackrel{8}{\circ}$ & $\stackrel{8}{\circ}$ \\
\hline$\stackrel{8}{\circ}$ & $\stackrel{8}{\circ}$ & $\stackrel{8}{0}$ \\
\hline$\stackrel{8}{\circ}$ & $\stackrel{8}{\circ}$ & $\stackrel{8}{\circ}$ \\
\hline$\stackrel{8}{\circ}$ & 8 & $\stackrel{8}{8}$ \\
\hline$\stackrel{8}{\circ}$ & $\stackrel{8}{\circ}$ & $\stackrel{8}{0}$ \\
\hline$\stackrel{8}{\circ}$ & $\stackrel{8}{\circ}$ & $\stackrel{0}{0}$ \\
\hline$\stackrel{\circ}{\circ}$ & $\stackrel{0}{0}$ & $\stackrel{8}{0}$ \\
\hline$\stackrel{8}{\circ}$ & $\stackrel{0}{0}$ & $\stackrel{8}{0}$ \\
\hline$\stackrel{8}{\circ}$ & $\stackrel{0}{0}$ & $\stackrel{8}{\circ}$ \\
\hline$\stackrel{8}{\circ}$ & $\stackrel{8}{0}$ & $\stackrel{8}{0}$ \\
\hline$\delta_{0}^{0}$ & $\stackrel{8}{0}$ & $\stackrel{8}{0}$ \\
\hline$\stackrel{\leftrightarrow}{\circ}$ & $\begin{array}{l}\stackrel{O}{0} \\
\stackrel{0}{0}\end{array}$ & $\stackrel{8}{\circ}$ \\
\hline$m$ & $\sim$ & - \\
\hline
\end{tabular}


苍 


\section{CHAPTER 3}

Measuring visual, spatial, and visual spatial short-term memory in schoolchildren: studying the influence of age, sex, and level of parental education and demographically corrected norms

Published as: Toornstra, A., Hurks, P. P. M., Van der Elst, W., Kok, G., \& Curfs, L. M. G. (2019). Measuring visual, spatial and visual spatial short-term memory in schoolchildren: Studying the influence of demographic factors and regression-based normative data. Journal of Pediatric Neuropsychology. doi 10.1007/s40817-019-00070-6 


\title{
Chapter 3
}

\begin{abstract}
The study aims to establish demographically corrected norms for three computerized tasks measuring different aspects of visual short-term memory (VSTM) in Ukrainian schoolchildren. These tasks measure respectively visual STM (the Pattern Recognition Memory (PRM) test), spatial STM (the Spatial Span (SSP) task), and visual spatial STM (the Paired Associates Learning (PAL) task). All tasks were administered to $n$ $=186$ children aged 5.10 years to 14.5 years old to evaluate the influence of demographic variables. Relevant demographic factors that influence task scores (VSTM), i.e., age and level of parental education, are identified and in keeping with the current literature. No sex differences were found. Based on these data, regression-based, demographically corrected norms were established per task. This approach to constructing norms differs from how (worldwide) PRM, SSP, and/or PAL norms have been constructed traditionally. In the latter approach, norms are calculated for each age group separately and without correcting for level of parental education, whereas in the regression-based normative method, multiple regression models are used to compute the expected test scores of an individual (rather than the subgroup means that are used in the traditional approach). Consequently, the regressionbased norms for the PRM, SSP, and PAL presented in this paper are individualized, taking into account the unique characteristics of the individual that is tested on these tasks. Last, the confidence intervals of the PRM scores of the Ukrainian schoolchildren and the Western norm group largely overlapped, except for the youngest age group, which adds to the literature about cultural effects on cognition.
\end{abstract}

Keywords: Visual (spatial) memory; Demographic influences; Continuous norms, schoolchildren; Ukraine 


\section{Introduction}

The Cambridge Neuropsychological Test Automated Battery $\left(\right.$ CANTAB $^{\circledR}$; Cambridge Cognition, 2012), is a test battery frequently used in both clinical and behavioral research. Three CANTAB ${ }^{\circledR}$ subtasks, i.e., the Pattern Recognition Memory (PRM) task, the Spatial Span (SSP) task, and Paired Associates Learning (PAL) task, are most often used to evaluate visual short-term memory (VSTM) in children. VSTM is typically defined as a storage system that holds limited amounts of information in mind for a brief period of time (Baddeley et al., 2011). VSTM is required in learning (Kessels et al., 2000). Hitch, Towse, and Hutton (2001) have shown that indeed VSTM is a predictor of success in school. Separate brain mechanisms are believed to be involved in the short-term storage of information from different modalities, i.e., within the domain of VSTM (e.g., visual, versus spatial information; Smith, Jonides, \& Kroeppe, 1996; Bigelow and Poremba, 2012). For instance, neurobiological research shows a ventral pathway in the brain, which is more concerned with processing and storing of colors and shapes (i.e., the features) of an object, and a dorsal pathway, which is involved in the processing and storage of locations of objects and/or their spatial relations (Gruhn \& Rauscher, 2002; Leng \& Shaw, 1991; Kravitz, Saleem, Baker, Ungerleider, \& Mishkin, 2013). The ventral (parvocellular) pathway is thought to involve the primary visual cortex and temporal brain areas associated with short- and long-term memory, emotions, reward, and learning (Kravitz et al., 2013). The dorsal (magnocellular) pathway is thought to be an occipital parietal network, which lies between the primary visual cortex and subcortical areas; is associated with e.g., visually guided action (including the (pre)motor cortex) and spatial navigation; and is involved in processing random and possibly dynamic relations between objects (i.e., their locations) (Kravitz et al., 2013). Simultaneously, features of an object (ventral processing) are thought to be, to some degree, incorporated in dorsal temporal processes to, e.g., guide decisions (i.e., not grasping a prickly-looking cactus). Indeed, the three CANTAB ${ }^{\circledR}$ subtasks, i.e., the PRM, the SSP, and the PAL, measure different aspects of VSTM: visual memory, spatial memory and visual-spatial memory respectively (see below for a detailed description of the tasks). In this study, the CANTAB ${ }^{\circledR}$ and specifically these subtasks were used to test these different components of non-verbal VSTM.

The PRM tests recall of abstract-colored patterns. The participant needs to identify the pattern that was previously shown (i.e., the target pattern) in a series of patterns (for an example, see Figure 1 in the 'Methods' section). The SSP is different from the PRM in that it requires the child to remember and to reproduce a location of the target and not the (visual) pattern of the target (spatial VSTM; for an example, see Figure 2). Finally, in the PAL, the child needs to remember and reproduce both the pattern and location of the target (see Figure 3). Administering and analyzing responses on the three tasks allows for a more complete understanding of brief VSTM in children; e.g., remembering a pattern is different from remembering a location.

These tests however, also implicate other cognitive functions associated with visual short-term memory, e.g., a general executive (Baddeley et al., 2011). Diamond 


\section{Chapter 3}

(2013) defined executive functions (EFs) as a set of independent yet interconnected cognitive skills that, among others, support one to meet demands of novel tasks, consider concepts from several perspectives and plan and execute solutions. Attention is an EF thought to underlie all other cognitive functions, and maturation of these EFs has been associated with development of prefrontal brain areas (Anderson, 2002). Alloway, Gathercole and Pickering (2006) found that domain general processes, e.g., the employment of attention, were more active in $4-6$ years old children in spatial VSTM tasks, which was thought to be related to the relative immaturity of these general cognitive functions.

Processes involving more than brief storage, e.g., the manipulation of information in the service of complex cognitive behaviors such as comparing and updating information are also theorized to be working memory (Fuster, 2002). It is beyond the scope of this article to discuss in-depth visual information processing and STM and/or working memory.

Importantly, results obtained on tests such as the PRM, the SSP, and/or the PAL are only meaningful when compared to appropriate norm data for a sample of schoolchildren (Lezak, Howieson, Bigler, \& Tranel, 2012). In the current study, normative data for these $\mathrm{CANTAB}^{\circledR}$ subtests were prepared for children living in the Ukraine. Even though normative data for these CANTAB ${ }^{\circledR}$ subtests have been published for schoolchildren living in, among others, countries such as, America, Australia, and Finland (De Luca et al., 2003; Lehto, Juujärvi, Kooistra, \& Pulkkinen, 2003; Luciana \& Nelson, 2002), this is not the case for a Ukrainian pediatric sample.

It is relevant to collect normative data for these tests for schoolchildren living in different countries, since cognitive functions, in turn have been associated with environmental factors such as culture. Thinking, knowledge, values, and beliefs are thought to constitute culture and are formed in social situations (Ardila, 2005). These variances in social and cultural contexts are linked to geographical differences. Ukraine, with its long and complex history may share cultural characteristics with other Slavic and/or former USSR countries (Subtelny, 2009). Variances in test performance could for instance arise due to different levels of familiarity with testing, including non-verbal tests (Ardila, 1995). Comparison of test scores of for, example Ukrainian children against Western norms (e.g., Western European and American norms as is mostly the case with the CANTAB ${ }^{\circledR}$ ) may help to identify differences and similarities between these two groups and is therefore included in this study (in line with e.g., Zasenko et al., 2017, and Toornstra et al., 2019).

As mentioned above, results of these tasks are only relevant when compared to suitable norm data (Lezak et al., 2012). Norm data presently available internationally for these CANTAB ${ }^{\circledR}$ subtests include several limits. These norms tend to be calculated based on means and standard deviations for relevant subgroups of children discretely, e.g., per two-year age band (De Luca et al., 2003; Lehto et al., 2003; Luciana and Nelson, 2002). Including age as a demographic factor in establishing norms is consistent with research. For example, multiple studies have shown that performances on numerous cognitive tests are influenced by the demographic variable 'age' (Lezak et al., 2012). There are to the best of our knowledge, relatively fewer studies on the PRM, SSP, and PAL tasks in children (De Luca et al., 2003; Luciana and Nelson 2002; Robbins et al., 1994; Sahakian and Owen, 
1992). Studies in children using these $\mathrm{CANTAB}^{\circledR}$ and comparable subtasks have shown that VSTM can be measured in children aged 5 years and older and age-related improvements can be observed with these tasks (Davidson, Amso, Anderson, and Diamond, 2006; De Luca et al., 2003; Green et al., 2019; Luciana and Nelson 2002). In addition, performances on (spatial) VSTM tasks, comparable to the PRM, SSP, and PAL tests, have been shown to increase with age, i.e., from early pre-school years well into middle school (De Luca et al., 2003; Luciana and Nelson, 2002).

However, calculating norms based on means and standard deviations for relevant subgroups of children separately, e.g., per two-year age bands, has its limitations (see e.g., Van der Elst, Hurks, Wassenberg, Meijs, \& Jolles, 2011). In calculating normative data, the total sample needs to be subdivided into several different subgroups based on, e.g., age. Four examples of the disadvantages of making these subgroups in the data are that (1) test scores are only applied to one particular (age) subgroup in the sample while (2) children closely in age (i.e., 1 month apart; e.g., 6.9 versus 7.0 years old) may be in different age subgroups. Additionally, (3) the (age) subgroups themselves may be subject to unusual sample characteristics but result in a norm for that (age) subgroup.

Another (4) limitation is that often other demographic characteristics (e.g., not only age, but also, e.g., sex, and the level of parental education (LPE)) are not included, or considered, while constructing norms. Including these demographic characteristics next to age, in the traditional way of norming described above would result in even more subgroups. These other demographic characteristics have been associated with interindividual differences in cognitive neuropsychological test performances in, e.g., adults (and participant's own level of education) including visual spatial memory (Heaton, Taylor, \& Manly, 2003; Norman et al., 2011). For instance, Voyer, Voyer, and Saint-Aubin (2017) conducted a meta-analysis regarding the effects of sex differences on performances on various (spatial) VSTM tests in 3- to 86-years old participants. They included, in total, 180 studies and found, overall, a small but significant effect in favor of males. However, when distinguishing between VSTM (pattern recognition as in the PRM) and memory for location (as seen in the SSP and PAL tasks), they found an effect in favor of females on tasks concerning memory for location. Furthermore, the demographic variable LPE has been studied relatively little in relation to VSTM in schoolchildren. LPE is perceived as an approximation of socio-economic status (SES) (Davis-Kean, 2005) and higher levels of both have been positively linked to an increase in children's cognitive functions in general and among others to VSTM specifically (Hackman et al., 2014; Kaplan et al., 2001; Noble, McCandliss, \& Farah, 2007). In addition, Noble and colleagues (2015) found, in their large sample (1099 participants from 3 - 20 years old) an association between SES and differences in children's brain structures (e.g., brain structures associated with memory performance and spatial skills). This showed especially when comparing the brains of children in the lower SES group to children from the other SES groups (adjusted for age and genetic factors). Differences in environment were hypothesized to be among others, family stress, limitations in cognitive stimulation and nutrition, and exposure to toxins of which children in lower SES environments might experience more. Taking into account 


\section{Chapter 3}

these unique characteristics (i.e., including age, but also sex and LPE) of the individual who is tested on the tasks, would make the norms more individualized in our opinion.

To circumvent the disadvantages of traditional norming methods mentioned above, we will employ in our study an alternative and promising method called continuous norming to collect PRM, SSP, and PAL normative data (in line with, e.g., Van Breukelen and Vlaeyen, 2005; Van der Elst et al., 2011; Zachary \& Gorsuch, 1985). This different method of norming is based on multiple regressions while weighing several demographic variables, such as age, sex, and LPE. In these regression analyses, both continuous variables (e.g., age in years) and categorical variables (e.g., sex or LPE) can be simultaneously incorporated, without creating subgroups for each variable (Van der Elst et al., 2011). Using age as a continuous variable means that the relation between age and the predicted score is calculated from all data points in the whole sample (i.e., combining children from all age groups in one analysis). This results in more refined estimates of agebased norm scores (Bechger, Hemker, \& Maris, 2009). Additionally, the possibility of incorporating more than one demographic variable in regression models makes this normative method rather individualized, as mentioned above (Van der Elst et al., 2011). These findings underline the relevance to study the effects of the demographic variables age, sex, and LPE, and establish the normal range and norm data for the PRM, SSP, and the PAL for this sample of Ukrainian schoolchildren $(n=186)$ age 5.10 to 14.5 years old.

\section{Methods}

\section{Participants}

The sample comprised of $n=186$ children enrolled in two local primary and middle schools in Ukraine. Per school all children of these schools were asked and $80.5 \%$ took part. Characteristics of this sample are displayed in Table 1. Age was defined as a continuous variable ranging from age 5.10 - 14.5 years. The level of parental education was established as the level of education completed by the parent(s) or guardians in a household; low was defined as education up till grade 9 (primary and middle school) and high was from grade 10 (high school) onward, in line with the Ukrainian educational system (Ukraine Channel, 2017). Education is compulsory up until and including grade 9. This is akin to Western ages of compulsory education, i.e., 16 years old. The highest level of education was assigned as the mean for the household in the $6.5 \%$ of the cases when there was a difference (high vs. low) between parents' (or caregivers') level of education (as in e.g., Koball and Jiang, 2018). Attending school beyond the compulsory years is, in general in Ukraine but even more in these rural areas linked to socio-economic status. High school and further education require children to travel further to larger institutes, which necessitates families to have or access means to do so. The kind of schooling parents attended has been linked to test scores in children (Ardila, Rosselli, Matute, \& Guajardo, 2005). 
Table 1: Demographic data of the sample

\begin{tabular}{|l|l|l|l|l|l|l|l|}
\hline & $n$ & $\begin{array}{l}\text { Age (Mean } \\
\text { in years) }\end{array}$ & Age SD & \multicolumn{2}{|l|}{ Sex } & \multicolumn{3}{|l|}{$\begin{array}{l}\text { Level of Parental } \\
\text { Education (LPE) }\end{array}$} \\
\cline { 3 - 8 } & & & & Female & Male & Low & High \\
\hline Total Sample & 186 & 9.63 & 2.49 & 90 & 96 & 23 & 163 \\
\hline
\end{tabular}

\section{Procedure}

Researchers informed schools through word of mouth in the area about the study. School management invited the parents to information meetings about the study. Information and consent letters were provided by school management and then taken home by the parents. Testing started after parents and children had provided their teachers with the informed consent letters. Testing was carried out during school hours and no compensation was provided.

As part of the CANTAB ${ }^{\circledR}$ (Cambridge Cognition, 2012), the PRM, the SSP, and the PAL were administered individually in a separate room according to the prescribed standardized procedure. The tests were administered on a HP Pavilion TS sleek-book laptop with a 15-inch touch screen. Two CANTAB ${ }^{\circledR}$-trained researchers administered the PRM, the SSP, and the PAL applying the predescribed script and practice items in the children's native language. Tests and outcome measures are described below.

The research ethics committee of the Faculty of Psychology and Neuroscience of Maastricht University, The Netherlands, approved this study. All data were collected according to ethics regulations of the World Medical Association Declaration of Helsinki (2013). In line with these ethic regulations the first researcher gave each child and his/her parents/guardians a brief individual report (stating the individual's number of items remembered, e.g., an encouragement to remember one more item over the next month and some general ideas to nurture brain development), while management received anonymous summaries of class and school achievements.

\section{Instrument}

The PRM is a VSTM task (Cambridge Cognition, 2012). Validity in children was established among others in school children aged 4-12 years old (Luciana and Nelson, 2002; Teixeira, Zachi, Roque, Taub, \& Ventura, 2011). Furthermore, a moderate test-retest reliability (.64 to .84 ) was found on $\mathrm{CANTAB}^{\circledR}$ tasks (including a test comparable to the PRM, the SSP, and the PAL; Lowe \& Rabbitt, 1998). For the PRM task, the procedure is as follows (Figure 1): First, abstract colored target patterns appear in the middle of the screen, one at a time on a black background. After a series is finished, two patterns (the target and a 


\section{Chapter 3}

distractor) appear on the screen. The participant needs to identify which of these two patterns was part of the previously presented target pattern, by touching it on the screen. Auditory and visual feedback is provided to indicate a(n) (in)correct response.
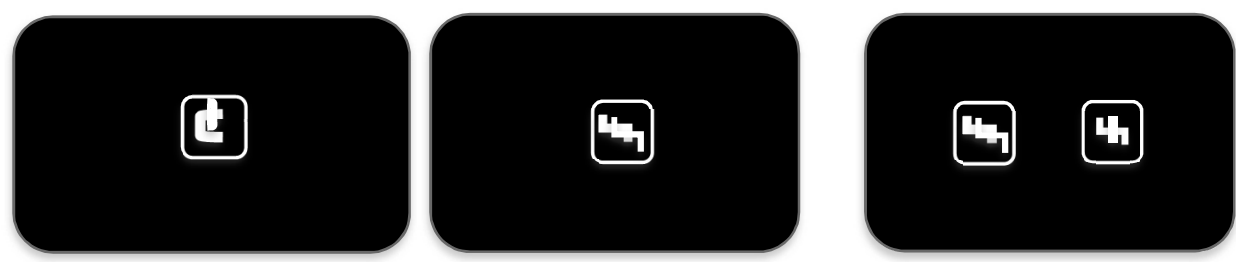

Figure 1. PRM on a touch screen: One series of 12 single patterns is followed by a forced choice recognition of the target patterns (this is not a real but similar CANTABß PRM sample).

The outcome measure PRM reports accuracy, i.e., how many patterns were recognized correctly. The participant completes two series of twelve trials in total, making the maximum accuracy score 24 correctly identified patterns. Total administration time is on average 4.5 minutes (Cambridge Cognition, 2012). In general, in these three CANTAB ${ }^{\circledR}$ tasks, touching boxes outside the designated squares or touching them twice (illegal responses), is not registered but only the first touch of a specific box.

The SSP is a computerized version of the Corsi Block Tapping task (see Milner, 1971), which is used to assess spatial (including temporal) VSTM. Support for the validity of the SSP in pediatric samples was for example found by Lehto et al. (2003) and Luciana and Nelson (2002). The procedure of this task is as follows (Figure 2): A number of randomly located boxes change into a particular color one after the other and then turn white again. The participant's task is to remember the location and exact order of boxes that changed color. He or she needs to touch the previously colored boxes in the same order shown earlier, after which the trial is finished.
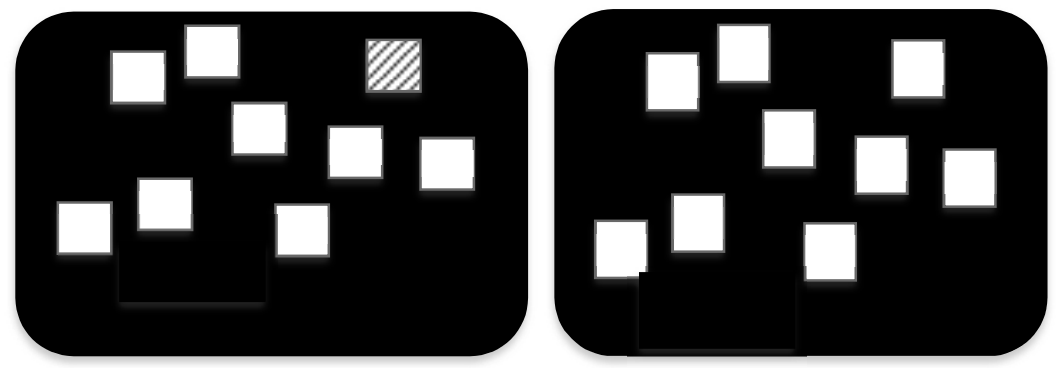

Figure 2. SSP on a touch screen: Showing one box that has changed color in this example trial of 3 boxes in total (this is not a real CANTAB ${ }^{\circledR}$ SSP sample). 
Each trial starts on a new black screen. Task difficulty rises with increasing span length by one block at a time, ranging from two to nine boxes. There are several difficulty levels depending on the number of boxes that change color. Per difficulty level, e.g., two boxes, subjects have three attempts to repeat the previously shown string of two. The test terminates in case of three erroneous responses at a certain difficulty level and continues to the next level after a correct response is completed. A trial is scored as correct if all boxes have been touched in the exact same order as previously shown. The outcome measure of the SSP is the total amount of cubes correctly located on the first attempt (i.e., first trial out of the possible three attempts) over all difficulty levels. The maximum SSP score is 44 boxes correct (i.e., 8 trials, starting at 2 boxes and increasing with one box each). Total administration time about 5.5 minutes (Cambridge Cognition, 2012).

The PAL is said to assess visual spatial STM. Validity of this test was found in schoolchildren (Luciana and Nelson, 1998, 2002).
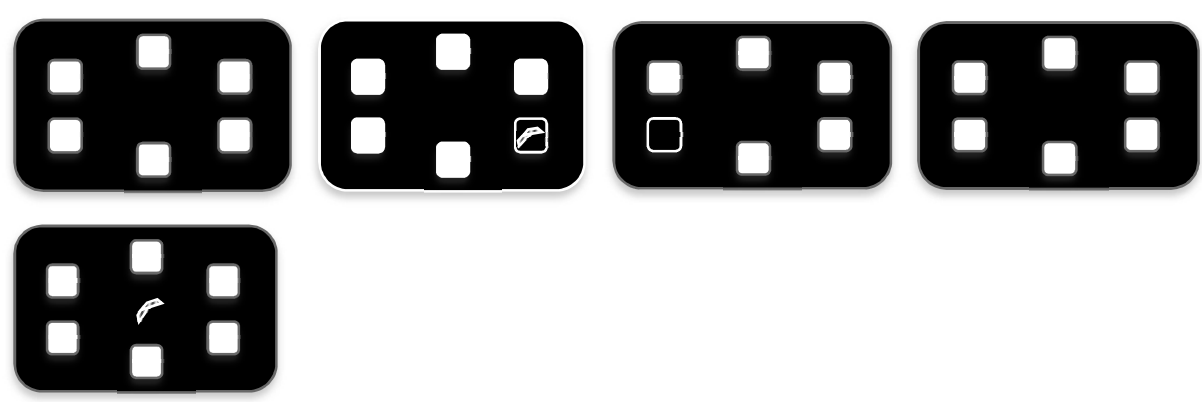

Figure 3. The PAL on a touch screen: a trial with a target pattern in the middle. The PAL increases in difficulty to 8 patterns (this is not a real CANTAB ${ }^{\circledR}$ PAL sample).

The procedure is as follows (Figure 3): White boxes are displayed in a circle on the screen, and one or more boxes contain an unique (hidden) pattern. A trial starts with one of the boxes opening and revealing a complex colored pattern. Then, the box closes, and a next box opens to reveal its pattern. Once all boxes are closed again, a target pattern appears in the middle of the screen. The participant needs to touch the box where the target pattern was located (and previously shown) in the trial. Then another target pattern appears, and the participant needs to locate this one and so forth. The trial ends when all patterns have been successfully located in one attempt. In case of an error, the whole trial (and thus all boxes) is registered as incorrect, and it is shown again with the target patterns; the participant needs to try the same trial from the beginning, which may be repeated up till a maximum of 10 times. As in the SSP, a trial is only scored as correct if all boxes are identified correctly. The test is terminated if the $10^{\text {th }}$ attempt is also incorrect. The test continues to the next level as soon as a correct trial is completed, and a new black screen appears with the number of white boxes for the particular level. The number of boxes opening - and thus, the difficulty level - increases over trials to a maximum of 8 boxes (i.e., the range consists 


\section{Chapter 3}

of 1-8 boxes). The outcome measure of the PAL is the number of patterns successfully located in the first attempt across difficulty levels. The maximum PAL score is 32 correctly identified patterns. Total administration time is on average 11 minutes (Cambridge Cognition, 2012).

\section{Statistical analyses}

Means and standard deviations were calculated for the PRM, the SSP, and the PAL (see Table 2). Then Pearson correlations among these PRM, SSP, and PAL outcome measures were computed (Table 3).

The regression analyses included the variables age, sex, and LPE. The categorical variables sex and LPE were dummy coded ( 0 female and 1 male; LPE low $=0$ and LPE high = 1). Also, all two-way interactions were included (e.g., age $\times$ LPE as an independent variable) as predictors that contribute to explaining the variance in the PRM, SSP, and/or PAL scores (van der Elst et al., 2011). Age was centered (Age_C = calendar age in months - mean age of the sample 115.58 months) to avoid multi-collinearity due to inclusion of power terms (Van der Elst, van Boxtel, van Breukelen, \& Jolles, 2005). Quadratic and cubic age were included in the analyses to ensure the best possible fit of the model (e.g., curvilinear) as opposed to a linear relation, i.e., allowing for a smaller increase in scores at certain ages and a larger increase in scores during other age periods (Shaw et al., 2006). Multiple linear regressions were fitted to the data, applying a step-down hierarchical method. Predictors with a significant value of $p<.05$ were included. The observed scores and expected scores (based on the regression models) were plotted and visually evaluated. More complex models allow for a better fitting model, e.g., in case of multiple curves in the data. To effectively model the potentially more complex curvilinear relation between the demographic variables and the test score at hand, fractional polynomials were fitted.

Fractional polynomials can be used when initial visual inspection of the data indicates a non-linear relationship i.e., the effect of a variable; e.g., age may be greater at a lower age compared to the effect in the middle age group children and change direction once more in older children. Adjusting for the possibility of multiple curves in the data resulted in choosing fractional polynomial models because the parameters for each curve can be more reliably estimated compared to multiple linear regressions (Van der Elst, Molenberghs, Hilgers, Verbeke, \& Heussen, 2016). For a better fit a restricted set of 7 exponents was used $\{-2,-1,-0.5,0,0.5,1,2\}$ with exponent $^{0}$ as the natural $\log$ (Van der Elst et al., 2016). Each of these values were used for the combinations of powers, while this restricted set has proven to reliably result in an optimally fitting regression model and prevents having to consider too many (multiples of hundreds) fractional polynomials (Van der Elst et al., 2016). The best model was selected among a proposed set of several models in the analysis based on the Akaike Information Criterion (AIC, i.e., lower indicates a better fit). Finally, the fit of the fractional polynomial models was again graphically evaluated against the observed scores, i.e., by adding the expected scores to the plot. 
The assumptions of regressions were tested for each model. Regression analysis is based on the assumption of a normal distribution of the residuals (Field, 2009). Visual inspection to assess this assumption of normality was carried out using P-plots of the residuals. Next, the normal distribution was tested using the Kolmogorov-Smirnov test on the residuals. Then the predicted values were divided in quartiles. These quartile groups were applied to the standardized residuals and Levene's test was used to assess homoscedasticity (Van der Elst et al., 2011). Multi-collinearity was evaluated using Variance Inflation Factors (VIF $\leq 10$ ), while Cook's distance and standardized residuals > $|3|$ of the mean, were calculated to identify influential cases (Fisher et al., 2014).

Finally, normative data based on the regression (fractional polynomial) models were calculated using the following procedure (Van Breukelen and Vlaeyen, 2005; Van der Elst et al., 2016). The expected scores were calculated using the final models. Then, the residuals were computed (= observed score - expected score). Next, the residuals were standardized by calculating residual / SD (residual) with SD (residual) $=$ the standard deviation of the residuals in the normative sample (Van der Elst et al., 2011). Finally, the residuals were converted into percentile values using the normal distribution (with mean = 0 and $S D=S D$ (residual)) if the assumption for normality of the standardized residuals was met in the normative sample (otherwise, the empirical distribution of the standardized residuals of the test score in this sample was used). The Appendix shows these calculated scores. The alpha level of .05 was used to avoid Type 1 errors. Analyses were carried out in SPSS (version 24) and R.

Last, the Ukrainian sample was compared to the Western norm to establish how the groups relate to each other. Individual scores and LPE were not available for the CANTAB $^{\circledR}$, so overall means of the two samples could only be compared in a descriptive manner, i.e., by establishing the confidence intervals (CIs) (Field, 2009). The mean child CANTAB ${ }^{\circledR}$ PRM observed score (Cambridge Cognition, 2014), based on its traditionally grouped two-year age brackets (i.e., 6-7 years old up till 13 - years old; see Figure 4) was compared to the same age group of the Ukrainian PRM sample. Only the PRM outcome measure was comparable to the CANTAB ${ }^{\circledR}$ norm group outcome measure. The SSP and PAL outcome measures used here were calculated to only include correct items on the first attempt in contrast to other studies, and could thus not be compared to the $\mathrm{CANTAB}^{\circledR}$ norm group (see materials; Green et al., 2019; Luciana and Nelson, 2002, Mattson et al., 2010).

\section{Results}

The means, standard deviations ( $S D)$ and confidence intervals (CIs) for the observed data of the PRM, the SSP, and the PAL are displayed in Table 2. Three children did not start the SSP, because they wished to terminate the test battery early. 
Table 2: Means and Standard Deviations of the observed scores for the PRM, the SSP, and the PAL amount correct

\begin{tabular}{|l|l|l|l|l|l|l|l|}
\hline & Mean & $\begin{array}{l}\text { Std. } \\
\text { Deviation }\end{array}$ & $\mathrm{n}$ & $\begin{array}{l}\text { Minimum of } \\
\text { the sample }\end{array}$ & $\begin{array}{l}\text { Maximum of } \\
\text { the sample }\end{array}$ & $\begin{array}{l}\text { Confidence } \\
\text { Interval }\end{array}$ & $\begin{array}{l}\text { Maximum } \\
\text { possible } \\
\text { test score }\end{array}$ \\
\hline PRM & 19.09 & 3.45 & 186 & 8 & 24 & $18.59-19.59$ & 24 \\
\hline SSP & 7.94 & 7.50 & 183 & 0 & 38 & $6.85-9.03$ & 44 \\
\hline PAL & 9.61 & 4.80 & 186 & 1 & 26 & $8.91-10.30$ & 32 \\
\hline
\end{tabular}

The comparison of the overall PRM means (observed scores) of the Ukrainian sample to the $C A N T A B \circledR$ norm groups included only the age groups which overlapped between the two populations, e.g., 6 - 7 years old and excluding the youngest and oldest age groups (Figure 4). The CIs for the means of the two samples overlapped for children from $8-13$ years old indicating that there is no significant difference between the means for the compared age groups (see Figure 4 for all CIs). However, the CIs of the means of the $6-7$ years old, of the CANTAB® (19.60-21.52) and Ukrainian (16.39-18.53) samples, did not overlap.

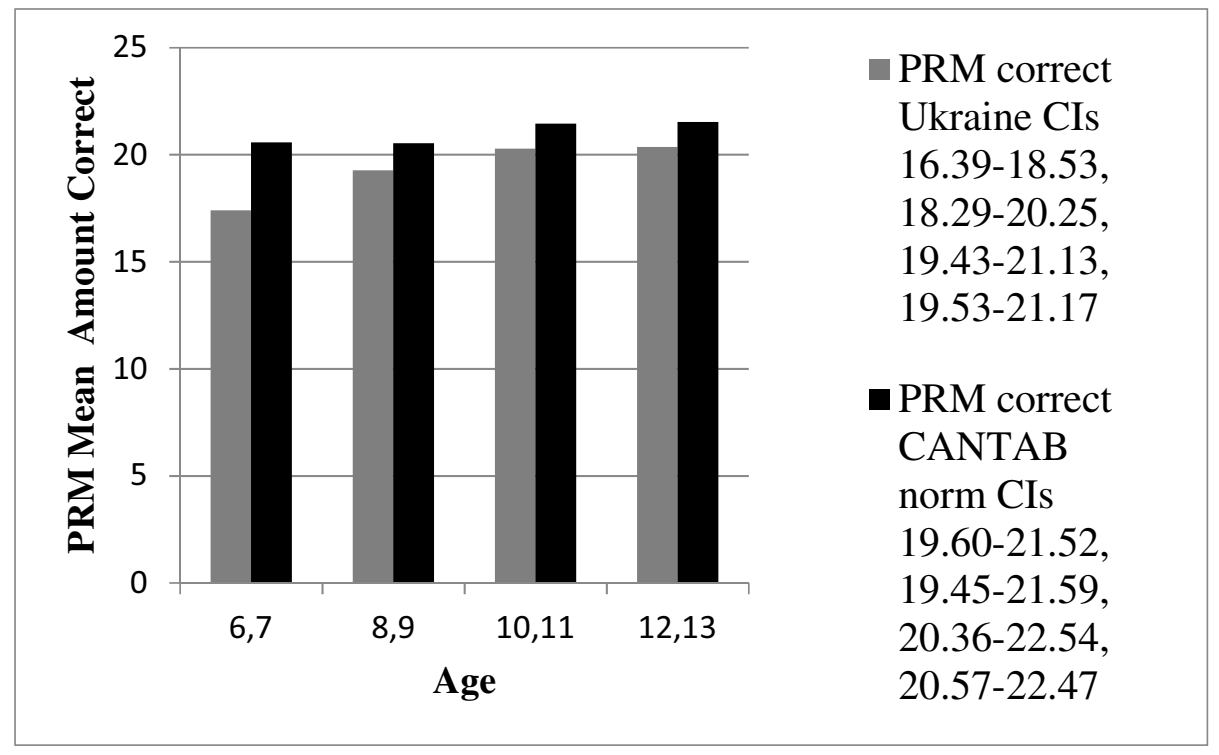

Figure 4. Observed scores for the mean PRM correct comparing the Ukrainian sample to the CANTAB ${ }^{\circledast}$ traditional mean norms (two-year age groups) including confidence intervals (CIs). 
Table 3 shows the correlations between these outcome measures for the Ukrainian sample. The PRM, the SSP, and the PAL outcome measures correlated significantly among each other; a higher accuracy on PRM correlated to a higher accuracy on the PAL for example. The correlations ranged from 0.22 to 0.38 , which is not high (i.e., .90 or higher). This indicates a significant relation between all three scores, but at the same time the PRM, the SSP, and the PAL appear to measure different components as well. This difference may be supported by the overlap in CIs of the SSP and PAL, but the CIs of both tests do not overlap with those for the PRM (Table 2), measuring accuracy on the first attempt (although test differences need to be kept in mind).

Table 3: Pearson zero order correlations for the PRM, SSP and PAL correct

\begin{tabular}{|l|l|l|l|}
\hline$n=186$ & PRM & SSP & PAL \\
\hline PRM & 1 & & \\
\hline SSP & $0.38^{* *}$ & 1 & \\
\hline PAL & $0.22 * *$ & $0.30^{* *}$ & 1 \\
\hline
\end{tabular}

** correlation is significant at the 0.01 level (2-tailed)

Table 4: Final regression models for the PRM, SSP, and PAL correct outcome measures

\begin{tabular}{|c|c|c|c|c|c|c|c|}
\hline Test score & $\mathrm{n}$ & Variable & B & $\mathrm{p}$ & $\mathrm{t}$ & $\begin{array}{l}\text { Std. } \\
\text { Deviation } \\
\text { (residual) }\end{array}$ & $\mathrm{R}^{2}$ \\
\hline \multirow{4}{*}{$\begin{array}{l}\text { PRM } \\
\text { (Box-Cox } \\
\text { transformation, } \\
\lambda 2.00 \text { ) }\end{array}$} & 186 & (Constant) & 72.27 & 0.00 & 9.20 & & \multirow[t]{4}{*}{$.16^{*}$} \\
\hline & & Age_C & 0.41 & 0.00 & 4.68 & & \\
\hline & & Age_C ${ }^{2}$ & -0.01 & 0.00 & -3.08 & & \\
\hline & & LPE & 17.11 & 0.03 & 2.21 & 34.13 & \\
\hline \multirow{4}{*}{$\begin{array}{l}\text { SSP } \\
\text { (Box-Cox } \\
\text { transformation, } \\
\lambda 0.85 \text { ) }\end{array}$} & $181 * *$ & (Constant) & 14.69 & 0.00 & 1.78 & & \multirow[t]{4}{*}{$.30 *$} \\
\hline & & $\mathrm{Age}^{3}$ & 0.001 & 0.00 & 2.65 & & \\
\hline & & $\mathrm{Age}^{3}$ (LNAge) & -0.0001 & 0.00 & -2.70 & & \\
\hline & & $\mathrm{Age}^{2}$ & -0.03 & 0.00 & -2.29 & 5.88 & \\
\hline
\end{tabular}




\begin{tabular}{|l|l|l|l|l|l|l|l|}
\hline PAL & $176^{* *}$ & (Constant) & 8.25 & 0.00 & 9.66 & & $.30^{*}$ \\
\cline { 2 - 7 } & & Age_C & 0.13 & 0.00 & 5.12 & \\
\cline { 2 - 7 } & Age_C & -0.0004 & 0.4 & -0.93 & \\
\cline { 2 - 7 } & Age_C & -0.00004 & 0.02 & -2.37 & \\
\cline { 2 - 6 } & LPE & 1.92 & 0.02 & 2.29 & 3.66 & \\
\hline
\end{tabular}

Note: Encoding of the predictors: Age_C $=$ Calendar Age in months - Mean Calendar Age in months 115.58, LPE (level of parental education).

** $\mathrm{N}$ is lower due to removal of outliers (residuals $>3$ Standard Deviations). $* \mathrm{p} \leq .03$.

Subsequently, the final regression (fractional polynomial) models $(p \leq .03)$ are shown in Table 4. Box Cox transformations were applied because initial analyses indicated heteroscedasticity in the observed scores of the PRM and the SSP (Osborne, 2010). Additionally, the PAL and SSP displayed 3 and 2 outliers respectively (residual Standard Deviation $>|3|$ ), that were removed. After this, assumptions for regressions were mostly met for the final models, i.e., all values of the Levene's statistic $p>.05$ except for SSP (Bartlett $p=.51$ ). Bartlett's test of sphericity is based on a likelihood ratio to make an estimate of the distribution (Filed, 2009). This test was more suitable for the SSP due to the distribution of the residuals showing a natural log and cubic age effect (Table 4).

Cook's distance values were $\leq .01$; all Variance Inflation Factors were $\leq 6.9$ (which is below the VIF cut-off value of 10 that indicates multi-collinearity). Kolmogorov-Smirnov values were $p \geq .01$, except for the SSP, $p<.001$. The assumption of normal distribution as assessed in the Kolmogorov-Smirnov test was not met for the SSP, despite visual inspection of residual plots showing reasonably normal distributions. Yet, to meet statistical assumptions, norms for the SSP were based on the distribution of the standardized residuals of the SSP test scores of the Ukrainian sample (i.e., the empirical distribution), as opposed to the theoretical standard normal distribution of residuals (Van der Elst et al., 2012). Sex did not influence test performance, which also shows when comparing the mean observed scores for females and males respectively, per task, i.e., PRM $n=96$ (mean 18.86), and $n=$ 90 (mean 19.33); SSP $n=88$ (mean 7.943), and $n=95$ (mean 7.937); PAL $n=90$ (mean 10.11), and $n=96$ (mean 9.14). Also, none of the interactions (e.g., Age_C $\times$ LPE) did reach significance.

For all outcome measures, there was a curvilinear effect (see Figure 5, based on the final norm calculations and if applicable reverted Box Cox transformations; Osborne, 2010). The PAL and SSP also showed a cubic age effect, indicating less steep development with age in the youngest age group. Highest levels for the PRM were achieved from 10.5 years old; for the SSP, from 12 years old; and for the PAL, from 12.5 years old, 
respectively. Accuracy started to level off from 14 years old in the PRM, and from 13 years old in the SSP and the PAL.

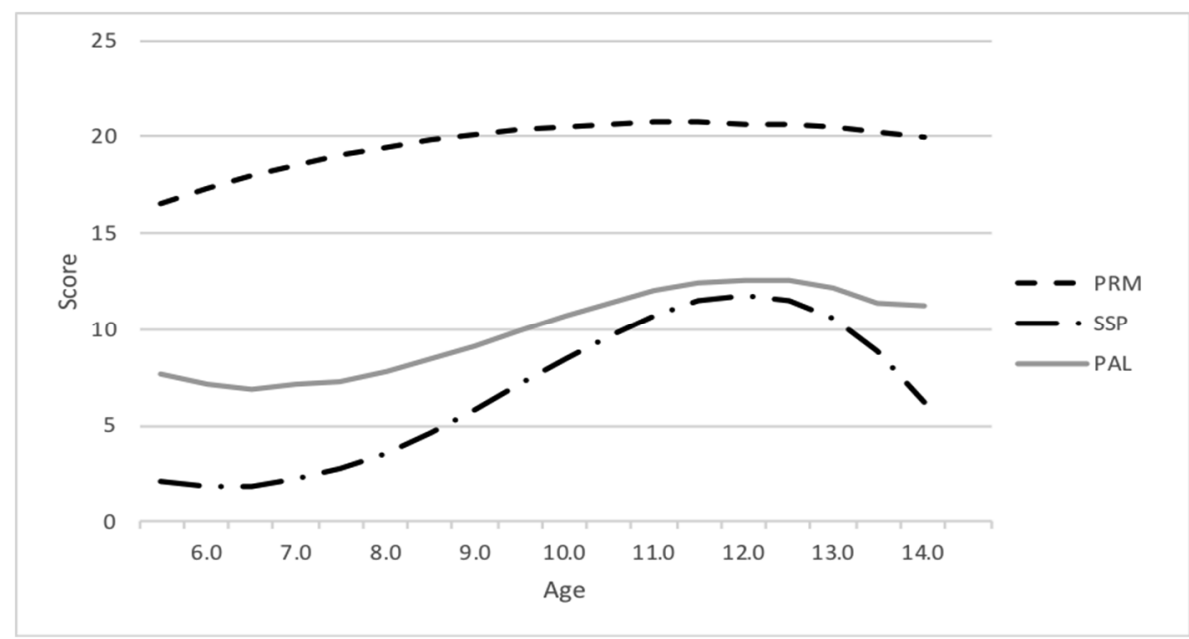

Figure 5. Results of the regression scores (Table 4) by age for the PRM (LPE high), SSP, and PAL (LPE high).

Last, the more detailed reporting of the SSP and the PAL (correct items on the first attempt only) allowed some comparison between the PRM, the SSP, and the PAL (i.e., visual, spatial and visual spatial memory). The maximum score on the PRM of 24 items was achieved by $4.8 \%$ of the Ukrainian schoolchildren. The maximum possible score was not achieved on the SSP and PAL; $0.6 \%$ scored 38 (out of 44) on the SSP and $1.7 \%$ of this sample scored 28 (out of 32) on the PAL. The CIs of the mean observed scores (Table 2) for the PRM (18.59 - 19.59) do not overlap with the SSP and the PAL $(6.85$ - 9.03; 8.91 10.30 respectively), confirming differences in score ranges.

\section{Normative procedure}

Norms for the PRM, the SSP, and the PAL correct outcome measures (Appendix Tables A1 - A3) are established using the procedure described above. For example, assume that a 6 years old child, whose parents have a high LPE, scored 6 points correct on the PAL. First in this normative procedure, the expected score for this child is calculated using the regression model presented in Table 4 . This is Constant $+[0.13 \times($ age child in months average age sample $)]+\left(-0.0004 \times\right.$ Age_C $\left.{ }^{2}\right)+\left(-0.00004 \times\right.$ Age_C $\left.^{3}\right)+(1.92 \times$ LPE $)$, that is $8.25+0.13 \times(72-115.72)+(-0.0004 \times 1911.44)+(-0.00004 \times-83568.09)+(1.92 \times 1)=$ 7.068. The residual is calculated, which is -1.068 (score $6-7.068$ ). Next, the residual is standardized, $-0.29(-1.068 / 3.66)$. The standardized residual is converted into a percentile value based on the normal distribution. A standardized residual of -0.29 corresponds to a 


\section{Chapter 3}

value of $45^{\text {th }}$ percentile. This means that $45 \%$ of the population of 6 years old children, whose parents have a high LPE obtain a PAL score that is equal or lower than 6 . The PAL score of this child is therefore in the lower part of normal limits $\left(5^{\text {th }}-95^{\text {th }}\right.$ percentile $)$. The SSP did not meet the normality assumption as evaluated in the Kolmogorov-Smirnov test. Norms were therefore based on the distribution of the standardized regression residuals (Van der Elst et al., 2012). The respective norm tables are in the Appendix Tables A1 - A3.

\section{Discussion}

The aim of the present study was to evaluate the effects of demographic variables on three tests measuring respectively visual, spatial and visual spatial short-term memory (STM) in school aged Ukrainian children. Also, normative data were established for this population by applying a promising method called continuous norming on these tests. This different approach to norming included the demographic variables age, sex, and level of parental education (LPE) and results in more adequate estimates of norm scores for reasons mentioned in the introduction (Van Breukelen and Vlaeyen, 2005; Van der Elst et al., 2011).

First, we found that all outcome measures on visual (PRM), spatial (SSP), and visual spatial (PAL) STM tasks correlated significantly, ranging from .22 to .38. The correlation indicates measurement of the same construct to some extent, yet it equally implies unique constructs, as the correlations are not high (i.e., $\geq .90$ ). Support for the correlation between visual, spatial, and visual spatial STM compared to, e.g., verbal STM was found by Smith et al. (1996). This model of domain specificity for visual (spatial) information was supported by Luck and Vogel (2013). Similarly, Alloway et al. (2006) found in children from 4 to 11 years old that brief memory processes rely on a central executive thought to be involved in domain general activities such as allocation of attention, while domain specific visual STM (VSTM) appears a system that represents and maintains visual, spatial and visual spatial information briefly (Baddeley et al., 2011; Luria, Sessa, Gotler, Jolicœur, and Dell'Acqua, 2009). Simultaneously, the far from perfect correlations find support in research distinguishing the brief storage of visual information in a visual- and a spatial network, i.e., the ventral (involved in processing of shape and colors of objects) and the dorsal pathway (implicated in processing spatial information), respectively (Courtney, Ungerleider, Keil, \& Haxby, 1996; Logie and Marchetti, 1991; Quinn, 1994; Rauscher et al., 1997). Moreover, the $\mathrm{SSP}$, requiring participants to remember a specific sequence of locations of squares, is thought to be a different task (associated with the dorsal pathway), i.e., a visual spatialsequential process (Mammarella, Pazzaglia \& Cornoldi, 2008).

Second, we found changes on test performance with age on all outcome measures, showing in principle increasing accuracy scores with maturation, albeit a curvilinear development (i.e., we found quadratic age effects on all outcome measures, Figure 5). Luciana and Nelson $(1998,2002)$ found age-related development on the PRM, the SSP, and the PAL in two samples of American children from 4 to 14+ years old. Most increases in accuracy appeared to be achieved in younger children (up until 8 years old; Luciana and 
Nelson 1998, 2002). Curvilinearity found in this study, also showed most increases in PRM scores with age in younger children and continued until about 11 years old. The overall mean scores of the Western PRM CANTAB ${ }^{\circledR}$ norms and the Ukrainian sample showed overlapping CIs for 8 - 13 years old children, which indicates a non-significant difference in the mean PRM scores between the two samples. However, the pattern of age related increases in SSP and PAL in this sample equally showed differences compared to some literature (De Luca et al., 2003; Luciana and Nelson, 1998, 2002), i.e., we observed cubic age effects (stabilizing scores) (Figure 5). These may relate to domain specific processes. Johnson, Mareschal and Csibra (2008) reviewed the evidence from infancy (i.e., younger children compared to e.g., Luciana \& Nelson (2002)) and concluded that refined development in the dorsal stream (spatial information processing) occurs later compared to the ventral stream, which in turn also implicates development of more complex connections between ventral and dorsal streams (Kravitz et al., 2013). The SSP is thought to test spatialsequential VSTM, i.e., a particular sequence of locations, which is associated with processing the location of objects in the dorsal pathway and the arbitrary and possibly dynamic relations between those processed objects (Kravitz et al., 2013; Mammarella et al., 2008). This applies to the PAL task as well, i.e., remembering the location of an increasing amount of target patterns per trial and related response items, and the covering of both target and response stimuli, imply a sequential process (Mammarella et al., 2008). Additionally, the PAL involves remembering matching pairs of target stimuli (VSTM for patterns) and their respective locations, thus implicating ventral and dorsal processing (Johnson et al., 2008; Kravitz et al., 2013). Furthermore, stabilizing scores in our study in younger children may implicate general executive functions (EFs) which according to Alloway et al. (2006) affect especially younger children in visual spatial tasks (Anderson, 2002). An environmental factor contributing to explaining the above suggested lower mean PRM score of younger children in the Ukrainian compared to the Western sample and literature, may be related to differences in school systems; compulsory education starts from 5 years old in, e.g., England and 6 years old in the Ukraine (Ukraine Channel, 2017). Last, development with age for spatial VSTM is proposed to continue into young adulthood (Farrell Pagulayan, Busch, Medina, Bartok, \& Krikorian, 2006; Luciana, Conklin, Hooper, \& Yarger, 2005; Luciana and Nelson, 2002), indicating a need for research including 15-21 years old.

The third finding concerns the demographic variable LPE, which is associated with socio-economic status (SES; Davis-Kean, 2005). A lower LPE (and thereby SES) background has been linked to decreased cognitive performances in children (Ardila et al., 2005; Bradley \& Corwyn, 2002, Koball \& Jiang, 2018). Higher LPE in this study is a significant positive predictor for PRM and PAL scores. This means that higher LPE is, in principle, associated with higher correct scores on tasks measuring visual and visual spatial STM (i.e., both tasks contain the detailed object features associated with parvocellular ventral processing). There were no effects on spatial STM (i.e., the SSP scores), which may support theory on distinguishing the ventral from the dorsal pathway (Kravitz et al., 2013). This is important because LPE is an environmental variable, and refined findings such as 


\section{Chapter 3}

these may help to identify specific areas in stages of development (i.e., the parvocellular object feature processing) that could benefit from intervention. On the other hand, finding no LPE effects on the SSP may contribute to research as well. Spatial VSTM is theorized to be associated with mathematics development in school, which in turn has been the subject of inconclusive findings (Alloway \& Passolunghi, 2011; Mix and Cheng, 2012; Raghubar, Barnes, and Hecht, 2010). Equally, learning is thought to become more fine-tuned after infancy and children learn to recognize which features are important to attend to, and allocate attention (i.e., employ executive functions) based on these selections (e.g., topdown processes; De Graaf, 2016) to support more complex pattern recognition tasks such as the PAL. It could be conceived that parents encouraging their children in these processes (e.g., playing memory games together) influence development. Researching the effects of LPE on (spatial) VSTM is thus, not only relevant for current norm scores, but also for identifying variables that may be linked to advantageous developmental pattern recognition. Last, the criterion for the high level LPE group (from grade 10 and higher) may appear relatively low, yet might have wider applicability. The National Center for Education Statistics (2014) for example, estimated that $7 \%$ of the US population did not obtain a high school diploma by age 24 years old.

The fourth finding is the absence of sex differences on these outcome measures. This is in line with several studies, which did not find sex differences on tasks measuring spatial VSTM (Farrell Pagulayan et al., 2006; Kessels et al., 2000; Luciana \& Nelson, 1998). Sex differences on spatial VSTM continue to be debated since Voyer et al. (2017) found in their meta-analysis an overall significant but small effect in visual spatial skills in favor of males (independent of age). They argued, however, that differences found, might be explained by moderator variables such as the task medium (i.e., computerized tests or remembering a pair of matched locations versus a sequence of locations), affecting the size and direction of effects in task subcategories in their review analysis of visual spatial development.

There are some limitations. The first concerns the sample size in this study. Indeed the sample size is relatively small, yet a range of studies used a similar sample size (e.g., Luciana and Nelson (2002) consisted of one school and a self-selected sample with on average $n=65$ in each age subgroup; De Luca et al. (2003), $n=194$ total, divided into age subgroups). The power of a test is defined as the probability that the null hypothesis of a significance test (like, e.g., $t$-tests to test the null $\mathrm{H} 0$ : beta $k=0$ in regression models) is rejected when the alternative is true (i.e., beta $k$ does not equal zero). In our models, significant effects of age and LPE were observed. This implies that the null hypotheses related to age and LPE were rejected and that there was therefore by definition sufficient power to conduct these tests. Furthermore, there were no sex differences, which is in line with several earlier studies (measuring visual, spatial and visual spatial STM (Farrell Pagulayan et al., 2006; Kessels et al., 2000; Luciana and Nelson, 1998) and thus supporting the evidence in this study.

The second limitation concerns sample characteristics. The high level LPE group was larger in numbers than the lower level LPE group. However, this is observed in more 
studies of norm data (Cherner et al., 2007; Norman, Evans, Miller, and Heaton, 2000). Regression based norms are thought to be a more accurate approximation of norms because the regression equations include the data for all demographic groups (as opposed to being based on a particular subgroup, e.g., lower LPE) (Van Breukelen and Vlaeyen, 2005). Not using subgroups but all scores in one regression analysis means statistically significant results can be achieved with fewer data. Furthermore, the sample consisted of public schools in rural areas. Towns fulfill a central role in Ukraine in that children travel to the largest one in an area, so schools are not necessarily smaller compared to urban schools. However, research including urban areas will increase generalizability as, e.g., Barona, Reynolds, and Chastain, (1984) found effects for urban-rural regions in children and adults with head trauma on the WAIS-R scores.

The third limitation concerns comparison to western norms, which was only carried out for the PRM (see results and Figure 4). Calculating for the SSP and PAL scores, the number correct on the first attempt enhanced comparing different forms of VSTM (e.g., not including the additional and varying number of attempts allowed and administered in this study per task). This may limit opportunities for comparison to other research or applicability of the norms, yet may also show how the described procedure of norming could be applied to scores on any of the outcome measures available in the three tasks. Further research is needed (beyond the descriptive measures (CIs) used) to establish (non-) significance of differences between the Western and our sample. However, the lack of overlap between the youngest age group of the two samples on the PRM might implicate early education. Compulsory schooling starts from 6 years old (Ukraine Channel, 2017). There are Kindergarten facilities for 5 years old children in Ukraine, but availability, schooling of educators and children's attendance does not fall under educational law.

Effects of schooling on cognitive development have been reported. Small but significant differences were found in cognition (albeit different tests) when comparing children from Flanders (Belgium) and The Netherlands for instance. These neighbouring countries share a similar language. Resing and Bleichroth (2015, p. 151) found a difference on subtests of the RAKIT intelligence test (6-12.5 years old) between the two nationalities, which among others, concerned processing and recognising patterns. Differences in findings in the WPPSI intelligence test in a sample from this mixed population, supported this claim; 4-7 years old Dutch children who habitually attend school from 4 years old, had higher scores than Flamish children of the same age (Hurks \& Hendriksen, 2015, p. 121). There were no differences in performance on the same tests in children younger than 4 years old. Results were explained primarily by differences in school systems; Kindergarten in Belgium (despite starting from 2.5 years old) focuses more on social and general school skills, whereas the focus in the Netherlands is more on cognitive skills (Schittekatte \& Hurks, 2015, p. 171). Neighbouring countries which may be perceived as similar (given the shared language) thus show differences in cognitive development at specific ages. The PRM showed lower scores in the youngest Ukrainain age group compared to the Western norm. If similar countries (Belgian Flanders and Holland) as discussed show differences in cognitive development in early years, then research might benefit from a focus on 


\section{Chapter 3}

differences in early years education including dissimilar populations such as western samples versus Ukraine. Additionally, this was a cross-sectional study. Longitudinal design would lend greater credence to future research as this may reveal the developmental trajectory of test scores in one sample (the same children) over time. Including younger children up until young adulthood is therefore recommended.

\section{Final conclusion}

Demographically corrected norms for three computerized VSTM tasks were established for a sample of Ukrainian schoolchildren. The regression-based analyses differs from traditional approaches to norming in allowing the inclusion of multiple demographic variables simultaneously. Age and level of parental education influenced VSTM task scores, while the demographic variable sex did not influence test performance in this sample. The regression-based normative method included all data points in the sample simultaneously and thus results in more suitable and individualized norms. Comparison of the VSTM task for patterns only, between the Ukrainian sample and the Western norm indicated no significant differences except for the youngest age group, which may point toward differences in early education. Demographically corrected norms increase the relevance of the interpretation of test scores for clinical settings. 


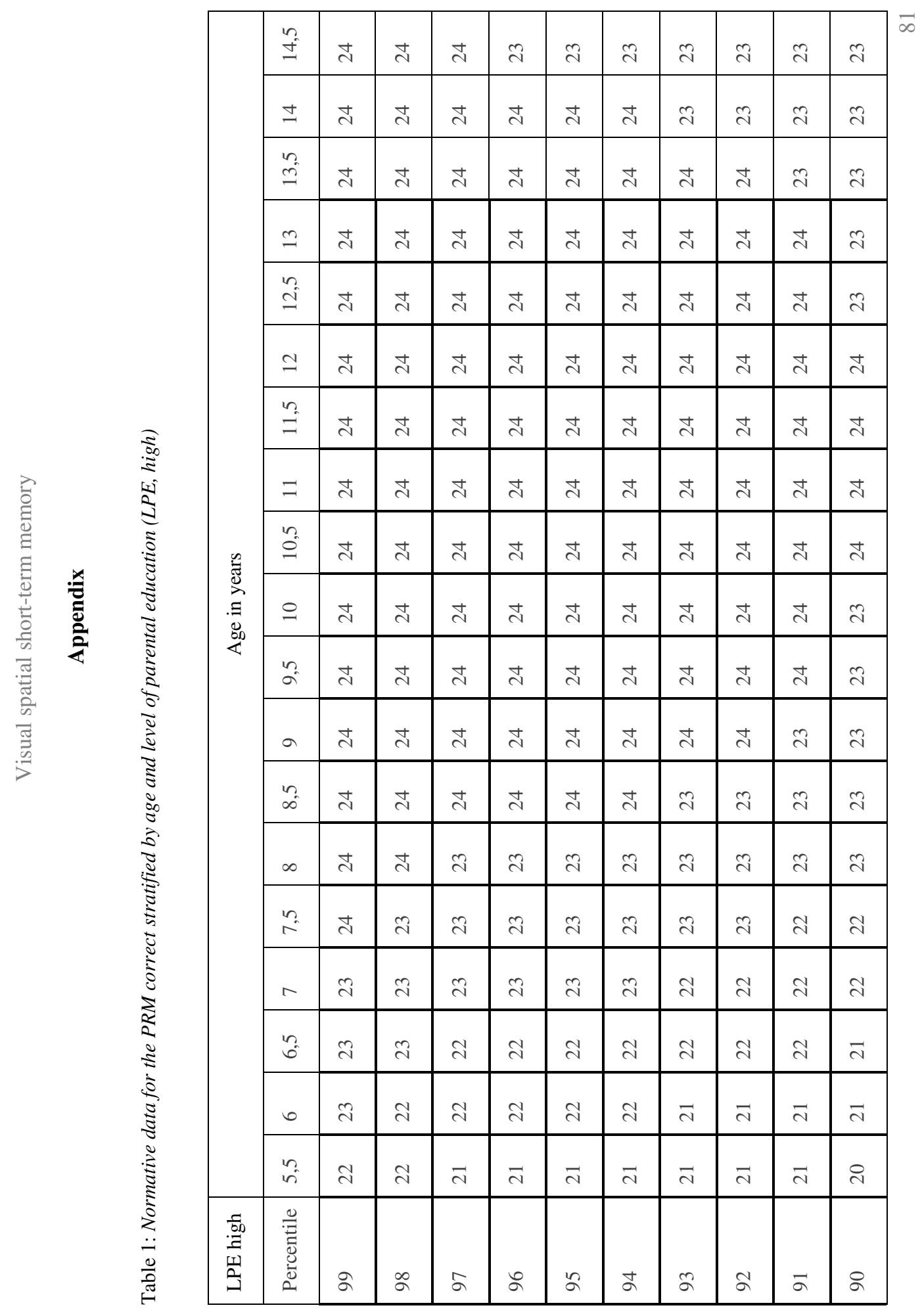




\begin{tabular}{|c|c|c|c|c|c|c|c|c|c|c|c|c|c|}
\hline$\tilde{\lambda}$ & $\ddot{\lambda}$ & ה & त & ה & ה & त & ה & $\tilde{N}$ & त & הี & i & $\Xi$ & 당 \\
\hline$\ddot{\sim}$ & $\ddot{\lambda}$ & $\ddot{\sim}$ & $\ddot{\lambda}$ & $\ddot{\sim}$ & $\ddot{\sim}$ & ป & $\widetilde{N}$ & $\widetilde{N}$ & $\approx$ & $\widetilde{\sim}$ & ㄱ & $\infty$ & 드 \\
\hline$\overparen{\sim}$ & $\ddot{\lambda}$ & $\ddot{\sim}$ & $\ddot{\sim}$ & $\ddot{\sim}$ & $\ddot{\sim}$ & $\ddot{\lambda}$ & $\widetilde{\sim}$ & $\tilde{\sim}$ & $\approx$ & $\widetilde{\sim}$ & 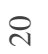 & $\cong$ & I \\
\hline$\overparen{\lambda}$ & $\tilde{\lambda}$ & $\vartheta$ & $\tilde{\lambda}$ & $\tilde{\lambda}$ & $\widetilde{\imath}$ & $\ddot{\lambda}$ & $\widetilde{\lambda}$ & $\widehat{\lambda}$ & $\approx$ & ปี & ㅇ & 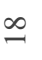 & $\stackrel{\infty}{=}$ \\
\hline 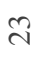 & $\tilde{\lambda}$ & $\approx$ & 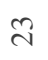 & $\tilde{\lambda}$ & $\tilde{\lambda}$ & $\ddot{\lambda}$ & 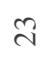 & $\widehat{\lambda}$ & $\approx$ & ปี & 요 & $\cong$ & $\stackrel{\infty}{=}$ \\
\hline$\widehat{\lambda}$ & $\tilde{\lambda}$ & $\ddot{\sim}$ & $\vec{\sim}$ & $\widehat{\sim}$ & $\ddot{\imath}$ & $\ddot{\lambda}$ & $\ddot{\sim}$ & $\widetilde{\sim}$ & $\ddot{\sim}$ & $\ddot{\imath}$ & $\vec{\sim}$ & $\cong$ & $\stackrel{\infty}{-}$ \\
\hline$\widetilde{\lambda}$ & $\tilde{\lambda}$ & $\ddot{\imath}$ & $\vec{\sim}$ & $\tilde{\sim}$ & $\ddot{\sim}$ & $\tilde{\lambda}$ & $\ddot{\sim}$ & $\widehat{\sim}$ & 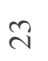 & $\ddot{\sim}$ & $\vec{\sim}$ & 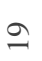 & $\stackrel{\infty}{-}$ \\
\hline$\overparen{\lambda}$ & $\tilde{\lambda}$ & $\ddot{\sim}$ & $\ddot{\sim}$ & $\widehat{\lambda}$ & $\tilde{\sim}$ & $\overparen{\lambda}$ & 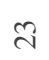 & $\overparen{\sim}$ & $\overparen{\lambda}$ & $\hat{\sim}$ & $\vec{\sim}$ & $\cong$ & $\stackrel{\infty}{\sim}$ \\
\hline$\overparen{\sim}$ & $\tilde{\lambda}$ & $\ddot{\imath}$ & $\ddot{\sim}$ & $\widehat{\sim}$ & $\widehat{\sim}$ & $\overparen{\lambda}$ & 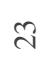 & $\overparen{\sim}$ & $\ddot{\lambda}$ & 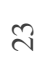 & $\vec{\sim}$ & $\cong$ & $\stackrel{\infty}{\sim}$ \\
\hline$\ddot{\sim}$ & $\widetilde{\lambda}$ & $\ddot{\sim}$ & $\ddot{\sim}$ & $\ddot{\lambda}$ & $\ddot{\imath}$ & $\ddot{\lambda}$ & 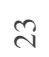 & $\overparen{\sim}$ & $\approx$ & ป & ‡ & $\stackrel{\infty}{-}$ & $\stackrel{\infty}{\simeq}$ \\
\hline$\widehat{\lambda}$ & $\tilde{\lambda}$ & $\ddot{\sim}$ & $\ddot{\sim}$ & $\tilde{\sim}$ & $\tilde{\sim}$ & $\tilde{\lambda}$ & $\ddot{\sim}$ & $\widetilde{\sim}$ & $\approx$ & ป & ㄱ & $\infty$ & $\stackrel{\infty}{-}$ \\
\hline$\widetilde{\lambda}$ & $\tilde{\lambda}$ & $\ddot{\sim}$ & $\ddot{\sim}$ & $\ddot{\lambda}$ & $\ddot{\imath}$ & $\ddot{\lambda}$ & $\ddot{\imath}$ & $\ddot{\lambda}$ & त & ป & 이 & $\cong$ & 드 \\
\hline$\widetilde{\lambda}$ & $\tilde{\lambda}$ & $\ddot{\sim}$ & $\ddot{\sim}$ & ป & ป & ป & ป & ป & ป & ป & ㅇ & $\stackrel{\infty}{-}$ & ㄷ \\
\hline ৯ & ป & ป & ป & ป & ป & ป & ป & ৯ & $\approx$ & ปี & $\stackrel{2}{2}$ & $\Xi$ & $\underline{-}$ \\
\hline 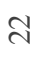 & ป & ป & ป & ป & ป & ป & ป & ป & $\approx$ & $\vec{\sim}$ & 2 & 근 & 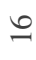 \\
\hline ป & ป & $\curvearrowright$ & $\vec{\sim}$ & $\vec{\sim}$ & $\vec{\sim}$ & $\vec{\sim}$ & $\vec{\sim}$ & $\vec{\sim}$ & $\vec{\sim}$ & $\vec{\sim}$ & $\infty$ & $\underline{0}$ & $\cong$ \\
\hline$\vec{\sim}$ & $\vec{\sim}$ & $\vec{\sim}$ & $\bar{\sim}$ & $\vec{\sim}$ & $\vec{\sim}$ & $\vec{\sim}$ & $\vec{\sim}$ & $\vec{\sim}$ & $\vec{\sim}$ & તి & $\infty$ & $\cong$ & \pm \\
\hline $\bar{\sim}$ & $\vec{\sim}$ & $\vec{\sim}$ & 요 & 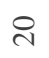 & ิ & ્ㅗ & ิㅗ & 이 & 이 & તి & 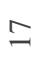 & \pm & $\cong$ \\
\hline ণ & ్ㅗ & ิ & 요 & ิ & 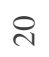 & ્ & ๙ิ & 이 & ㄱ & 9 & $\varrho$ & $m$ & $\simeq$ \\
\hline$\infty$ & $\infty$ & $\infty$ & $\infty$ & $\mathscr{\infty}$ & ఫ্ & $\tilde{\infty}$ & స్ & $\bar{\infty}$ & 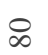 & $\curvearrowleft$ & in & $\approx$ & ㄱ \\
\hline
\end{tabular}




\begin{tabular}{|c|c|c|c|c|c|c|c|c|c|c|c|c|c|}
\hline I & 0 & 0 & 0 & 0 & 0 & 으 & 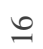 & $n$ & $n$ & $\because$ & 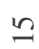 & $\because$ & 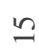 \\
\hline$\beth$ & 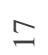 & $\underline{0}$ & 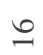 & 0 & 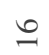 & 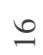 & $\varrho$ & 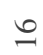 & 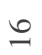 & 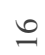 & $\because$ & $\because$ & 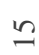 \\
\hline ㄱ & I & I & 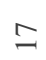 & 드 & 그 & 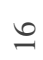 & $\stackrel{0}{0}$ & 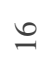 & $\stackrel{0}{-}$ & $\underline{0}$ & $\stackrel{0}{0}$ & $\underline{0}$ & $\stackrel{\bullet}{0}$ \\
\hline 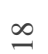 & I & I & $=$ & 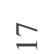 & 드 & 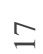 & $I$ & 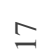 & I & $\underline{0}$ & $\underline{0}$ & $\underline{0}$ & $\stackrel{\bullet}{\sigma}$ \\
\hline$\infty$ & 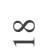 & $=$ & $=$ & 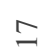 & 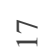 & 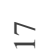 & $I$ & $I$ & I & 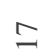 & $\underline{v}$ & $\underline{0}$ & $\stackrel{\bullet}{\sigma}$ \\
\hline$\infty$ & $\stackrel{\infty}{.}$ & 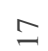 & 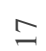 & 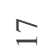 & $\therefore$ & 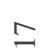 & $=$ & $=$ & $=$ & $=$ & 0 & 0 & $\mathscr{\sigma}$ \\
\hline$\infty$ & $\infty$ & 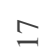 & $\therefore$ & 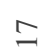 & 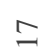 & 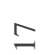 & $=$ & $=$ & $=$ & $=$ & $=$ & 0 & $\stackrel{0}{0}$ \\
\hline$\infty$ & 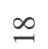 & $I$ & $\therefore$ & 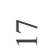 & 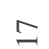 & 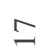 & $\therefore$ & 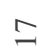 & 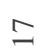 & $=$ & $I$ & 0 & 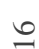 \\
\hline$\stackrel{\infty}{\sim}$ & $\stackrel{\infty}{\sim}$ & 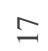 & 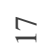 & 든 & 드 & 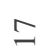 & I & I & 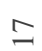 & 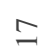 & 0 & 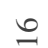 & $\underset{\sigma}{0}$ \\
\hline$\infty$ & $I$ & 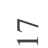 & 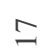 & $=$ & $\therefore$ & $\therefore$ & 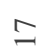 & 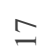 & $\therefore$ & 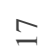 & 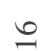 & 0 & 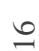 \\
\hline$\infty$ & $I$ & 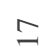 & 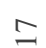 & 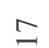 & 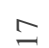 & 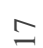 & 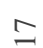 & 0 & 0 & 0 & 0 & 0 & 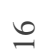 \\
\hline 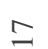 & 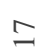 & $=$ & 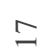 & $\approx$ & 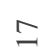 & 0 & 0 & 0 & 0 & 0 & $\underline{\sigma}$ & 0 & $\cong$ \\
\hline 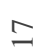 & 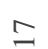 & 0 & 0 & 0 & 0 & 0 & 0 & 0 & 0 & $\underline{0}$ & $n$ & $\cong$ & $n$ \\
\hline$\underline{\sigma}$ & 0 & 0 & 0 & 0 & 0 & 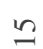 & 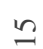 & $\because$ & 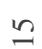 & $\because$ & $n$ & \pm & \pm \\
\hline$\underline{\ddots}$ & 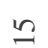 & $n$ & $\cong$ & 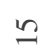 & $\cong$ & $n$ & $n$ & 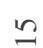 & \pm & \pm & \pm & \pm & \pm \\
\hline 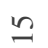 & 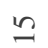 & $\Xi$ & \pm & \pm & \pm & \pm & \pm & \pm & \pm & 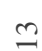 & 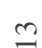 & 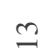 & $\stackrel{m}{2}$ \\
\hline$\Xi$ & $\Xi$ & 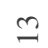 & $m$ & 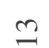 & 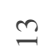 & 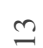 & $m$ & 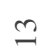 & 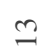 & $\simeq$ & $\simeq$ & $\simeq$ & $=$ \\
\hline$\underline{n}$ & $\underline{n}$ & $\simeq$ & $\simeq$ & $\simeq$ & $\simeq$ & $\simeq$ & $=$ & $=$ & $=$ & $=$ & 으 & $a$ & $a$ \\
\hline$\simeq$ & $=$ & 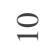 & $\circ$ & $ㅇ$ & 으 & $a$ & $a$ & $\infty$ & 6 & 0 & $\nabla$ & $\nabla$ & $m$ \\
\hline 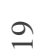 & $\infty$ & I & 0 & $\cong$ & \pm & $\cong$ & $\simeq$ & $=$ & 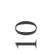 & $a$ & $\infty$ & $N$ & 6 \\
\hline
\end{tabular}




\begin{tabular}{|c|c|c|c|c|c|c|c|c|c|c|c|c|c|}
\hline \pm & \pm & 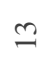 & 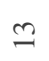 & $\simeq$ & \multirow{19}{*}{ 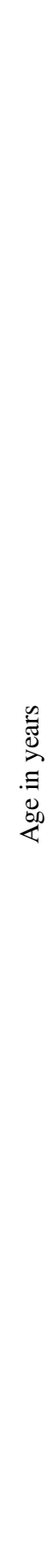 } & $\begin{array}{l}n \\
\pm\end{array}$ & $\stackrel{\sim}{\sim}$ & $\ddot{d}$ & 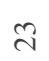 & $\ddot{\sim}$ & $\widetilde{N}$ & $\approx$ & ป \\
\hline$\cong$ & \pm & $\Xi$ & \pm & 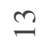 & & \pm & $\stackrel{\sim}{\sim}$ & 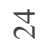 & $\ddot{\sim}$ & $\ddot{\sim}$ & $\ddot{\sim}$ & $\approx$ & ป \\
\hline 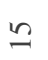 & $\underline{n}$ & $\Xi$ & \pm & 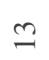 & & $\underline{n}$ & $\stackrel{\sim}{\sim}$ & $\stackrel{\sim}{\sim}$ & 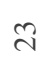 & $\ddot{\sim}$ & 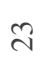 & $\ddot{\sim}$ & $\widetilde{\sim}$ \\
\hline$\stackrel{0}{ }$ & 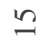 & $\stackrel{n}{n}$ & 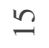 & \pm & & $\stackrel{n}{2}$ & $\stackrel{\sim}{d}$ & 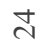 & ন & $\approx$ & 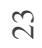 & $\approx$ & $\tilde{\sim}$ \\
\hline$\varrho$ & $n$ & $\stackrel{n}{n}$ & 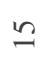 & \pm & & $\stackrel{n}{\simeq}$ & $\stackrel{\sim}{\sim}$ & 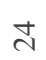 & $\stackrel{\sim}{\sim}$ & $\ddot{\sim}$ & $\partial$ & 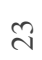 & 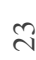 \\
\hline$\stackrel{\bullet}{-}$ & $\stackrel{0}{-}$ & 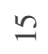 & $\bumpeq$ & \pm & & $\simeq$ & $\underset{\sim}{\sim}$ & $\stackrel{\Delta}{d}$ & $\stackrel{\Delta}{\Delta}$ & $\stackrel{ \pm}{d}$ & $\ddot{\sim}$ & $\ddot{\sim}$ & $\ddot{\sim}$ \\
\hline$\stackrel{\bullet}{0}$ & $\underline{-}$ & $\stackrel{n}{n}$ & $\cong$ & \pm & & 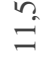 & $\stackrel{\sim}{\sim}$ & $\stackrel{\sim}{\sim}$ & $\stackrel{\sim}{d}$ & $\stackrel{\sim}{\sim}$ & $\ddot{\sim}$ & 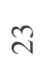 & $\widehat{\sim}$ \\
\hline$\stackrel{\bullet}{0}$ & $\stackrel{0}{-}$ & 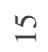 & $\cong$ & \pm & & $\exists$ & $\underset{\sim}{\sim}$ & $\stackrel{+}{\sim}$ & $\stackrel{+}{\sim}$ & $\stackrel{+}{\sim}$ & 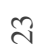 & $\ddot{\sim}$ & $\widetilde{\lambda}$ \\
\hline$\stackrel{\bullet}{-}$ & 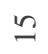 & $\cong$ & $\cong$ & \pm & & $\stackrel{0}{0}$ & $\stackrel{\sim}{\sim}$ & $\stackrel{\sim}{\sim}$ & $\stackrel{\sim}{\sim}$ & $\vec{\lambda}$ & $\vec{\sim}$ & $\ddot{\lambda}$ & $\tilde{\sim}$ \\
\hline$\varrho$ & $\mathfrak{n}$ & 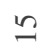 & $n$ & $\Xi$ & & 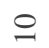 & $\stackrel{d}{d}$ & 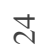 & $\stackrel{d}{d}$ & $\ddot{\sim}$ & $\ddot{\sim}$ & 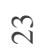 & $\widetilde{\sim}$ \\
\hline 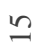 & 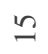 & 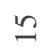 & \pm & \pm & & $\tilde{n}^{2}$ & $\stackrel{\Delta}{d}$ & $\stackrel{\Delta}{d}$ & $\stackrel{d}{\Delta}$ & $\ddot{\imath}$ & 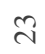 & $\ddot{\sim}$ & $\widetilde{\sim}$ \\
\hline$\cong$ & 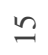 & \pm & \pm & $\mathscr{m}$ & & $a$ & $\stackrel{\Delta}{\sim}$ & $\stackrel{d}{d}$ & $\ddot{\sim}$ & $\tilde{\sim}$ & $\tilde{\lambda}$ & $\tilde{\lambda}$ & $\widetilde{N}$ \\
\hline 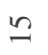 & \pm & \pm & $\mathscr{m}$ & 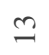 & & $\begin{array}{l}n \\
\infty\end{array}$ & $\stackrel{\Delta}{\sim}$ & $\ddot{\sim}$ & $\tilde{\lambda}$ & $\tilde{\lambda}$ & $\theta$ & $\widetilde{\sim}$ & $\widetilde{N}$ \\
\hline \pm & 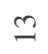 & 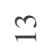 & $\underline{n}$ & $\simeq$ & & $\infty$ & $\stackrel{\Delta}{d}$ & $\ddot{\sim}$ & $\vec{\lambda}$ & $\vec{\lambda}$ & $\widetilde{N}$ & $\approx$ & $\widetilde{N}$ \\
\hline 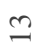 & $\underline{M}$ & $\simeq$ & $\simeq$ & 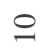 & & $\stackrel{n}{\sim}$ & $\ddot{\lambda}$ & $\theta$ & 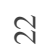 & $\widetilde{\sim}$ & $\widetilde{\sim}$ & $\widetilde{\sim}$ & $\widetilde{N}$ \\
\hline$\simeq$ & $=$ & $=$ & 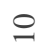 & 0 & & $r$ & $\ddot{\lambda}$ & $\approx$ & 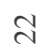 & ป & $\approx$ & $\vec{\sim}$ & $\vec{\sim}$ \\
\hline$=$ & & $\infty$ & $n$ & $m$ & & $\tilde{n}^{n}$ & $\ddot{\sim}$ & ป & ป & $\vec{\sim}$ & $\bar{\sim}$ & $\vec{\sim}$ & $\bar{\sim}$ \\
\hline 6 & 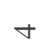 & $m$ & $m$ & $N$ & & 0 & $\approx$ & $\approx$ & $\vec{\sim}$ & $\vec{\sim}$ & $\bar{v}$ & ๙ิ & ¿ \\
\hline$m$ & $N$ & $N$ & - & 0 & & $n$ & ปี & $\bar{\sim}$ & $\vec{\sim}$ & 요 & ㄱ. & ๙ & 2 \\
\hline$n$ & $\nabla$ & $m$ & $N$ & - & $\frac{3}{\frac{3}{0}}$ & 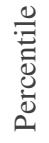 & ฉे & $\stackrel{\infty}{\circ}$ & á & ๙๐ & $\curvearrowleft$ & む゙ & ๙ \\
\hline
\end{tabular}




\begin{tabular}{|c|c|c|c|c|c|c|c|c|c|c|c|c|c|}
\hline ปี & ปี & 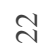 & $\vec{\sim}$ & $\bar{\sim}$ & $\vec{\sim}$ & $\vec{\sim}$ & $\vec{\sim}$ & $\vec{\sim}$ & $\vec{\lambda}$ & $\bar{\sim}$ & $\bar{\sim}$ & $\bar{\sim}$ & ㄱ \\
\hline ป & ปี & $\approx$ & तี & $\widetilde{N}$ & ה & $\vec{\sim}$ & $\vec{\sim}$ & $\vec{\sim}$ & $\vec{\sim}$ & $\bar{\sim}$ & $\bar{\sim}$ & $\bar{\sim}$ & ㄱ \\
\hline ปี & ป & ป & ปี & ป & ત & ป & ป & $\bar{\lambda}$ & $\bar{\lambda}$ & $\vec{\sim}$ & $\vec{\sim}$ & $\vec{\sim}$ & $\vec{\sim}$ \\
\hline$\ddot{\lambda}$ & ปี & ป & ปี & ป & ป & ป & ป & ป & ป & $\vec{\sim}$ & $\vec{\sim}$ & $\vec{\sim}$ & $\vec{\sim}$ \\
\hline$\approx$ & $\tilde{\lambda}$ & ป & ปี & ป & ป & ปี & ป & ป & ปี & ป & $\vec{\sim}$ & $\vec{\sim}$ & $\vec{\sim}$ \\
\hline$\ddot{\sim}$ & $\ddot{\sim}$ & $\tilde{\imath}$ & ป & ป & $\approx$ & ป & $\approx$ & $\widetilde{N}$ & ป & ป & $\approx$ & $\widetilde{N}$ & $\bar{\sim}$ \\
\hline$\ddot{\sim}$ & $\ddot{\imath}$ & $\tilde{\imath}$ & ป & ป & $\widetilde{\beth}$ & ปี & $\approx$ & ป & ปี & ป & ป & $\approx$ & $\vec{\sim}$ \\
\hline$\ddot{\sim}$ & $\ddot{\imath}$ & $\widehat{\imath}$ & $\widetilde{\sim}$ & ป & त & ป & $\approx$ & त & त & $\approx$ & $\approx$ & $\approx$ & $\bar{\sim}$ \\
\hline$\ddot{\sim}$ & $\tilde{\lambda}$ & ป & ปี & ป & ป & ป & त & त & ה & $\widetilde{N}$ & $\approx$ & $\bar{\sim}$ & $\vec{\sim}$ \\
\hline$\ddot{\sim}$ & $\ddot{\sim}$ & ป & ปี & ป & ป & ป & $\approx$ & तี & ปี & $\widetilde{\sim}$ & $\vec{\sim}$ & $\bar{\sim}$ & $\vec{\sim}$ \\
\hline ป & ป & ป & ปี & ป & $\approx$ & ป & ป & ป & $\vec{\sim}$ & $\bar{\sim}$ & $\vec{\sim}$ & $\bar{\sim}$ & $\bar{\sim}$ \\
\hline ป & ป & ป & ป & ป & ป & ป & $\vec{\sim}$ & $\bar{\lambda}$ & $\vec{\sim}$ & $\vec{\sim}$ & $\vec{\sim}$ & $\vec{\sim}$ & $\vec{\sim}$ \\
\hline ป & ป & ป & ป & ป & $\vec{\sim}$ & $\vec{\sim}$ & $\vec{\sim}$ & $\bar{\lambda}$ & $\vec{\sim}$ & $\vec{\sim}$ & $\vec{\sim}$ & $\vec{\sim}$ & ㄱ \\
\hline ปี & ป & $\vec{\sim}$ & $\bar{\sim}$ & $\bar{\lambda}$ & $\vec{\sim}$ & $\vec{\sim}$ & $\vec{\sim}$ & $\bar{v}$ & $\vec{\sim}$ & $\bar{\sim}$ & 이 & 이 & ¿ \\
\hline$\vec{\sim}$ & $\vec{\sim}$ & $\vec{\sim}$ & $\bar{\sim}$ & $\bar{\sim}$ & $\vec{\sim}$ & $\vec{\sim}$ & ㄱ & 오 & ㅇ & ㅇ & 이 & 이 & ㄱ \\
\hline$\vec{\sim}$ & $\bar{v}$ & $\vec{\sim}$ & $\bar{\sim}$ & 요 & 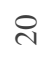 & 오 & ㄱ. & 요 & ㅇ & 이 & 이 & 이 & 9 \\
\hline ㅇ & 요 & 요 & 요 & 요 & 이 & 요 & 이 & $\stackrel{2}{2}$ & $\stackrel{2}{-}$ & $\stackrel{2}{2}$ & $\stackrel{2}{ }$ & 2 & 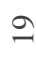 \\
\hline ㅇ & ㅇ & ㄱ. & $\stackrel{2}{ }$ & 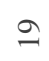 & 9 & 9 & 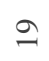 & 9 & 9 & $\stackrel{2}{2}$ & $\infty$ & $\stackrel{\infty}{\infty}$ & $\infty$ \\
\hline 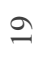 & 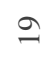 & 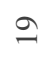 & $\cong$ & $\stackrel{2}{2}$ & $\stackrel{2}{ }$ & $\stackrel{\infty}{-}$ & $\stackrel{\infty}{\sim}$ & $\stackrel{\infty}{-}$ & $\stackrel{\infty}{-}$ & $\stackrel{\infty}{-}$ & $\stackrel{\infty}{-}$ & $\stackrel{\infty}{\sim}$ & I \\
\hline ส & $\bar{a}$ & ๙ & $\infty$ & $\infty$ & $\hat{\infty}$ & $\infty$ & $n$ & ‡ & $\tilde{\infty}$ & న & $\vec{\infty}$ & $\infty$ & $\approx$ \\
\hline
\end{tabular}




\begin{tabular}{|c|c|c|c|c|c|c|c|c|c|c|c|c|c|}
\hline$\stackrel{\infty}{\sim}$ & $\stackrel{0}{-}$ & 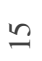 & 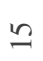 & 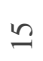 & $n$ & 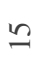 & \pm & $\Xi$ & $\Xi$ & \pm & 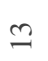 & $\stackrel{m}{2}$ & 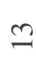 \\
\hline 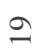 & $\stackrel{0}{-}$ & $\stackrel{0}{-}$ & $\stackrel{0}{-}$ & $\cong$ & 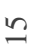 & $\cong$ & $\cong$ & 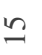 & $\cong$ & \pm & \pm & $\Xi$ & \pm \\
\hline$\cong$ & I & $\mathscr{0}$ & 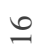 & 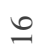 & 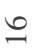 & $\mathscr{0}$ & $\bumpeq$ & $\bumpeq$ & $\bumpeq$ & $\cong$ & $\cong$ & $\Xi$ & \pm \\
\hline$\cong$ & I & 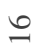 & $\mathscr{0}$ & 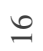 & $\underset{\imath}{0}$ & 0 & $\underline{\sigma}$ & $\underline{-1}$ & 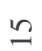 & $\cong$ & $\because$ & $\cong$ & \pm \\
\hline$\cong$ & ㄷ & I & I & 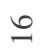 & 0 & 0 & $\underline{\sigma}$ & 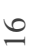 & 0 & 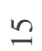 & $\because$ & 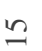 & $\because$ \\
\hline$\cong$ & I & I & I & $\beth$ & $\underline{\ddots}$ & 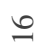 & $\underline{0}$ & 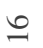 & 으 & 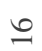 & 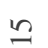 & $\cong$ & 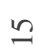 \\
\hline$\cong$ & I & I & 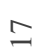 & $I$ & $\mathscr{\ddots}$ & $\underline{1}$ & $\underline{\ddots}$ & 0 & 0 & 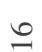 & $n$ & 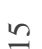 & 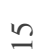 \\
\hline 2 & I & I & I & $I$ & $\underline{0}$ & 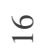 & $\mathscr{0}$ & 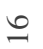 & 으 & $\because$ & 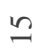 & 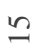 & $n$ \\
\hline$\cong$ & ㄷ & I & 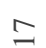 & 0 & 0 & 0 & 0 & 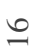 & 0 & $\cong$ & $n$ & 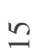 & $n$ \\
\hline$\cong$ & I & I & 0 & 0 & 0 & 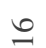 & $\underline{\ddots}$ & 0 & 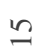 & $\cong$ & 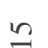 & 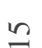 & $n$ \\
\hline$\cong$ & I & 0 & 0 & 0 & 0 & $\underline{\ddots}$ & 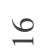 & 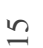 & 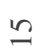 & $\cong$ & 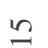 & $\cong$ & \pm \\
\hline$\cong$ & I & $\underline{0}$ & $\underline{0}$ & 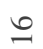 & $\underline{0}$ & $\cong$ & 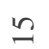 & 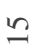 & 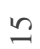 & $\cong$ & $\Xi$ & $\Xi$ & $\Xi$ \\
\hline$\stackrel{\infty}{\sim}$ & $\underline{0}$ & 0 & 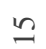 & 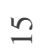 & $n$ & $\because$ & 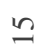 & 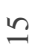 & む & $\Xi$ & $\Xi$ & $\Xi$ & $\underline{\sim}$ \\
\hline$\infty$ & 0 & 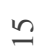 & 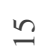 & 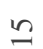 & $\underline{n}$ & \pm & \pm & \pm & \pm & 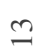 & $\cong$ & 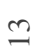 & 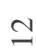 \\
\hline 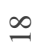 & 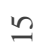 & 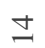 & $\Xi$ & $\Xi$ & $\Xi$ & $\Xi$ & $\underline{\sim}$ & $\cong$ & 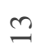 & $\stackrel{m}{=}$ & $\simeq$ & $\simeq$ & $=$ \\
\hline$\beth$ & $\Xi$ & $\stackrel{n}{\sim}$ & $\stackrel{n}{=}$ & $\stackrel{M}{=}$ & 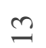 & 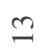 & $\simeq$ & $\simeq$ & $\simeq$ & $=$ & $=$ & 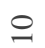 & 으 \\
\hline 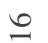 & 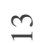 & 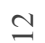 & $\simeq$ & 工 & I & $=$ & $=$ & $=$ & 으 & $a$ & $a$ & 6 & $n$ \\
\hline$\cong$ & $\simeq$ & $=$ & 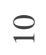 & 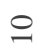 & 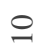 & $a$ & $\infty$ & 0 & in & 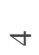 & $\nabla$ & $m$ & $m$ \\
\hline \pm & 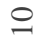 & 0 & $n$ & $\nabla$ & $\nabla$ & $m$ & $m$ & $m$ & 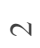 & $N$ & N & - & - \\
\hline in & $\approx$ & 오 & 9 & $\infty$ & $I$ & $\because$ & $\cong$ & \pm & 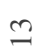 & 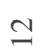 & $=$ & 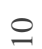 & $a$ \\
\hline
\end{tabular}




\begin{tabular}{|c|c|c|c|c|c|c|c|}
\hline$\simeq$ & $\simeq$ & $=$ & $=$ & $a$ & $n$ & $m$ & - \\
\hline 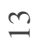 & $\cong$ & $\simeq$ & $\simeq$ & $=$ & $a$ & $\nabla$ & $N$ \\
\hline$\Xi$ & $\cong$ & $\cong$ & $\simeq$ & $\simeq$ & $=$ & $\infty$ & $m$ \\
\hline$\Xi$ & \pm & $\cong$ & $\stackrel{m}{ }$ & $\simeq$ & $=$ & $a$ & $m$ \\
\hline \pm & \pm & $\Xi$ & $\underline{n}$ & $m$ & I & 으 & $\nabla$ \\
\hline$\stackrel{n}{n}$ & \pm & $\Xi$ & $\underline{n}$ & 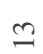 & I & $=$ & $n$ \\
\hline$\cong$ & \pm & \pm & $m$ & $\cong$ & $\simeq$ & $=$ & $n$ \\
\hline 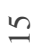 & \pm & $\Xi$ & $\stackrel{m}{\sim}$ & $\cong$ & $\simeq$ & $=$ & $n$ \\
\hline$\Xi$ & $\Xi$ & $\Xi$ & $\stackrel{m}{=}$ & $\cong$ & $\simeq$ & $ㅇ$ & $\nabla$ \\
\hline \pm & \pm & $\Xi$ & $m$ & $\simeq$ & $\simeq$ & 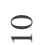 & $\nabla$ \\
\hline \pm & \pm & 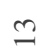 & $\stackrel{m}{=}$ & $\simeq$ & $=$ & $a$ & $m$ \\
\hline$\underline{-}$ & 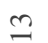 & $m$ & $\simeq$ & $=$ & 으 & 0 & $\mathrm{~N}$ \\
\hline 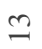 & I & $\simeq$ & $=$ & $\stackrel{0}{0}$ & $a$ & $\nabla$ & $\mathrm{N}$ \\
\hline$\simeq$ & $\simeq$ & $=$ & 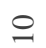 & $\infty$ & $\nabla$ & $m$ & - \\
\hline$=$ & $\varrho$ & $a$ & 0 & 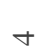 & $m$ & $N$ & 0 \\
\hline$a$ & 0 & 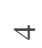 & $m$ & $m$ & $N$ & - & 0 \\
\hline$\nabla$ & $m$ & $m$ & $N$ & - & - & 0 & 0 \\
\hline N & N & - & - & 0 & 0 & 0 & 0 \\
\hline- & 0 & 0 & 0 & 0 & 0 & 0 & 0 \\
\hline$\infty$ & $r$ & 6 & $n$ & $\nabla$ & $m$ & $N$ & - \\
\hline
\end{tabular}

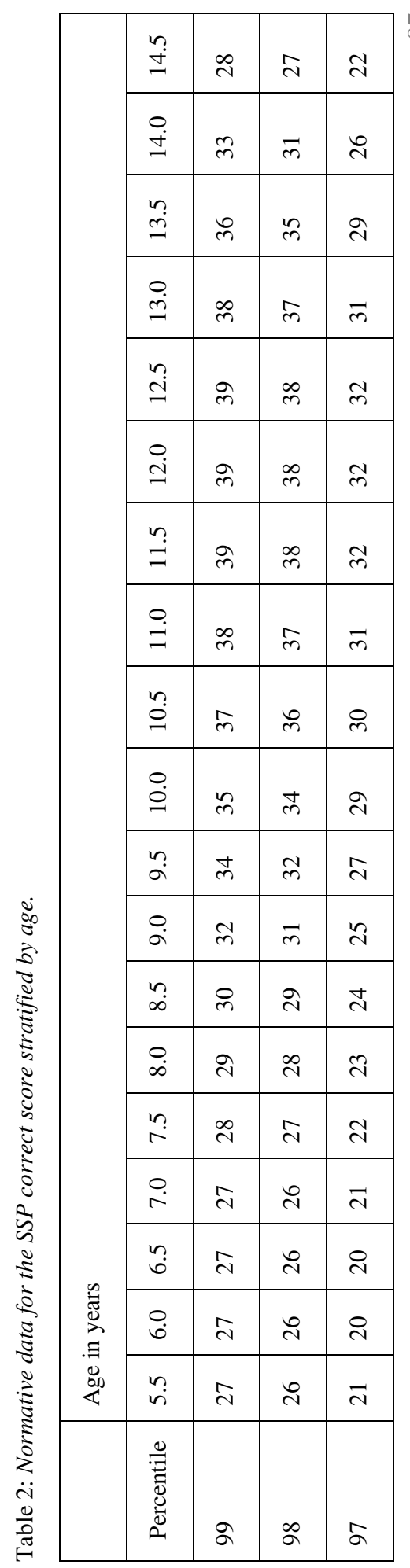




\begin{tabular}{|c|c|c|c|c|c|c|c|c|c|c|c|c|c|}
\hline ¿ & $\curvearrowright$ & 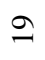 & $\stackrel{\infty}{-}$ & $\stackrel{\infty}{\sim}$ & $=$ & $\stackrel{0}{0}$ & $\stackrel{0}{0}$ & $\simeq$ & \pm & $\simeq$ & $=$ & $=$ & 으 \\
\hline$\stackrel{J}{d}$ & $\theta$ & $\ddot{\lambda}$ & ส & $\approx$ & $\vec{\lambda}$ & $\vec{N}$ & तิ & $\stackrel{2}{ }$ & $\stackrel{\infty}{\sim}$ & $=$ & 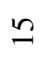 & 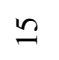 & $\Xi$ \\
\hline$\hat{\lambda}$ & $\hat{\lambda}$ & i & $\ddot{\imath}$ & $\ddot{a}$ & $\stackrel{J}{\sim}$ & $\stackrel{d}{4}$ & $\lambda$ & $\approx$ & $\bar{\sim}$ & 2 & $\stackrel{\infty}{.}$ & $\stackrel{\infty}{\sim}$ & 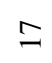 \\
\hline$\stackrel{\lambda}{ }$ & নิ & $\stackrel{\infty}{\sim}$ & $\hat{\imath}$ & $\hat{\lambda}$ & i & i & $\leadsto$ & $\stackrel{J}{\sim}$ & $\ddot{\sim}$ & $\vec{\lambda}$ & तิ & ¿ & $\stackrel{a}{ }$ \\
\hline i & i & ৯े & $\stackrel{\infty}{\sim}$ & $\stackrel{\infty}{\sim}$ & $\hat{\imath}$ & $\hat{v}$ & $\stackrel{2}{i}$ & $\ddot{a}$ & $\stackrel{J}{\sim}$ & ส & $\bar{\lambda}$ & $\vec{\sim}$ & iి \\
\hline $\bar{m}$ & iి & in & ते & $\stackrel{\infty}{\sim}$ & $\stackrel{\infty}{\sim}$ & $\hat{\imath}$ & $\hat{\imath}$ & $\stackrel{i}{\sim}$ & $\stackrel{\Xi}{d}$ & $\ddot{\lambda}$ & $\vec{\lambda}$ & $\vec{\sim}$ & ¿ి \\
\hline in & iి & ते & $\stackrel{\infty}{\sim}$ & $\stackrel{\infty}{\sim}$ & $\grave{\imath}$ & $\hat{\lambda}$ & $\stackrel{\sim}{N}$ & $\ddot{\sim}$ & $\underset{\sim}{J}$ & $\tilde{\lambda}$ & $\vec{\lambda}$ & $\bar{\sim}$ & ¿ \\
\hline i & ন̀ & $\stackrel{\infty}{\sim}$ & $\stackrel{\infty}{\sim}$ & $\hat{\lambda}$ & $\hat{\imath}$ & i & $\dddot{i}$ & $\stackrel{\Xi}{\sim}$ & $\ddot{\lambda}$ & ส & i & ㄱ. & $\stackrel{2}{2}$ \\
\hline$\stackrel{\infty}{\sim}$ & $\stackrel{\infty}{\sim}$ & $\hat{\lambda}$ & i & $\stackrel{i}{i}$ & $\ddot{v}$ & $\ddot{n}$ & $\dot{d}$ & $\ddot{\lambda}$ & $\tilde{d}$ & तิ & $\stackrel{2}{ }$ & $\stackrel{2}{ }$ & $\stackrel{\infty}{-}$ \\
\hline$\hat{\imath}$ & $\stackrel{\sim}{\sim}$ & $\stackrel{\sim}{\sim}$ & $\ddot{\imath}$ & $\stackrel{J}{\sim}$ & I & $\tilde{\lambda}$ & $\ddot{\lambda}$ & $\tilde{\sim}$ & $\vec{\sim}$ & 2 & $\stackrel{\infty}{-}$ & $\stackrel{\infty}{\sim}$ & $=$ \\
\hline$\check{\lambda}$ & $\ddot{\imath}$ & $\stackrel{\sim}{\sim}$ & 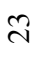 & 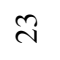 & $\widetilde{N}$ & $\tilde{\mathrm{N}}$ & $\bar{\sim}$ & 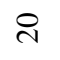 & $\stackrel{2}{2}$ & $\stackrel{\infty}{-}$ & 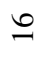 & $\stackrel{\bullet}{0}$ & $\cong$ \\
\hline$\stackrel{J}{\sim}$ & $\ddot{\imath}$ & $\ddot{\nu}$ & $\tilde{N}$ & $\bar{\sim}$ & $\vec{N}$ & iి & i & $\stackrel{2}{2}$ & $\stackrel{\infty}{\sim}$ & $\stackrel{0}{0}$ & 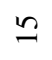 & 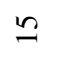 & $\Xi$ \\
\hline$\approx$ & $\vec{\sim}$ & $\vec{\lambda}$ & $\gtrsim$ & ¿ & 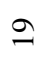 & 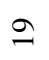 & $\stackrel{\infty}{.}$ & $=$ & $\stackrel{0}{ }$ & $\cong$ & $\cong$ & $\underline{n}$ & $\simeq$ \\
\hline$\vec{\sim}$ & ¿ & 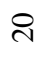 & $\stackrel{2}{ }$ & $\stackrel{2}{2}$ & $\stackrel{\infty}{=}$ & $=$ & 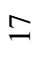 & $\stackrel{\bullet}{0}$ & $\cong$ & $\underline{2}$ & $\simeq$ & $\simeq$ & $=$ \\
\hline i & $\stackrel{2}{ }$ & 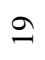 & $\stackrel{\infty}{-}$ & $\stackrel{\infty}{\sim}$ & $=$ & $\stackrel{0}{0}$ & $\stackrel{0}{0}$ & $\simeq$ & \pm & $\simeq$ & $=$ & $=$ & $\circ$ \\
\hline$\stackrel{2}{ }$ & $\stackrel{\infty}{\stackrel{-1}{2}}$ & $\stackrel{\infty}{\sim}$ & $\simeq$ & $=$ & $\stackrel{0}{0}$ & $\stackrel{0}{0}$ & $\cong$ & \pm & $\underline{n}$ & $\simeq$ & $=$ & $=$ & 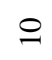 \\
\hline$\stackrel{2}{2}$ & $\stackrel{\infty}{\sim}$ & $\stackrel{\infty}{\mathscr{0}}$ & 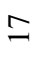 & $\stackrel{\bullet}{ }$ & $\stackrel{0}{0}$ & $\because$ & $\because$ & \pm & $\mathscr{m}$ & $=$ & 은 & 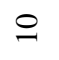 & $a$ \\
\hline$\stackrel{2}{ }$ & $\stackrel{\infty}{\infty}$ & $\stackrel{\infty}{\longrightarrow}$ & $\simeq$ & $\stackrel{\bullet}{=}$ & $\stackrel{0}{0}$ & $\cong$ & $\because$ & \pm & $\underline{m}$ & $=$ & 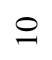 & 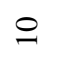 & $a$ \\
\hline 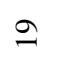 & $\stackrel{\infty}{\sim}$ & $\stackrel{\infty}{\sim}$ & $=$ & $\simeq$ & $\stackrel{0}{0}$ & $\stackrel{0}{0}$ & $\cong$ & \pm & $\cong$ & $\simeq$ & $=$ & $=$ & $\stackrel{0}{0}$ \\
\hline 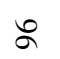 & $n$ & d & $\hat{\alpha}$ & 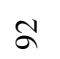 & $\bar{a}$ & \& & $\infty$ & $\infty$ & $\hat{\infty}$ & $\infty$ & $\ddot{\infty}$ & $\underset{\infty}{+}$ & $\infty$ \\
\hline
\end{tabular}




\begin{tabular}{|c|c|c|c|c|c|c|c|c|c|c|c|c|c|}
\hline 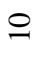 & 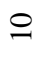 & $\stackrel{0}{=}$ & $\infty$ & $m$ & 0 & 0 & 0 & 0 & 0 & 0 & 0 & 0 & 0 \\
\hline \pm & \pm & \pm & $\simeq$ & 0 & $N$ & - & - & - & 0 & 0 & 0 & 0 & 0 \\
\hline$\beth$ & $\stackrel{0}{ }$ & $\mathscr{0}$ & $\cong$ & $a$ & $\nabla$ & $n$ & $m$ & $m$ & $N$ & $N$ & $N$ & $N$ & $N$ \\
\hline$\stackrel{2}{ }$ & $\stackrel{\infty}{\sim}$ & $\stackrel{\infty}{\sim}$ & 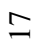 & $=$ & 0 & $n$ & $n$ & $\nabla$ & $\nabla$ & $\nabla$ & $m$ & $n$ & $m$ \\
\hline ㄱ & 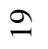 & 9 & $\stackrel{\infty}{-}$ & $=$ & 0 & 0 & $n$ & in & $\nabla$ & $\nabla$ & $\nabla$ & $\nabla$ & $\nabla$ \\
\hline$\stackrel{\sim}{ }$ & ণ্ণ & ㄱ. & $\stackrel{\infty}{-}$ & $\simeq$ & $r$ & 0 & 6 & $n$ & $n$ & $n$ & $\nabla$ & $\nabla$ & $\nabla$ \\
\hline 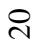 & $\curvearrowright$ & 9 & $\stackrel{\infty}{-}$ & $=$ & 0 & 0 & $n$ & $n$ & $\nabla$ & $\nabla$ & $\nabla$ & $\nabla$ & $\nabla$ \\
\hline 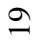 & $\curvearrowright$ & $\stackrel{\infty}{-}$ & $\beth$ & $=$ & 0 & $n$ & $n$ & $n$ & $\nabla$ & $\nabla$ & $m$ & $n$ & $m$ \\
\hline$\stackrel{\infty}{-}$ & 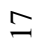 & $I$ & $\stackrel{\varrho}{ }$ & $\stackrel{0}{ }$ & $n$ & $\nabla$ & $\forall$ & $\forall$ & $n$ & $m$ & $m$ & $n$ & $N$ \\
\hline$\stackrel{0}{0}$ & 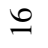 & $\mathscr{0}$ & 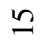 & $\infty$ & $\nabla$ & $m$ & $m$ & $m$ & $N$ & $N$ & $N$ & $N$ & - \\
\hline 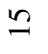 & $\cong$ & $\cong$ & $\cong$ & $r$ & $\sim$ & $\sim$ & $N$ & - & - & - & 0 & 0 & 0 \\
\hline$\cong$ & $\cong$ & 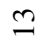 & $\simeq$ & 0 & - & - & 0 & 0 & 0 & 0 & 0 & 0 & 0 \\
\hline$\simeq$ & $\simeq$ & $\simeq$ & 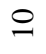 & $n$ & 0 & 0 & 0 & 0 & 0 & 0 & 0 & 0 & 0 \\
\hline$=$ & $=$ & $=$ & $a$ & + & 0 & 0 & 0 & 0 & 0 & 0 & 0 & 0 & 0 \\
\hline 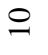 & 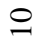 & $\stackrel{0}{ }$ & $\infty$ & $m$ & 0 & 0 & 0 & 0 & 0 & 0 & 0 & 0 & 0 \\
\hline$a$ & $a$ & $a$ & $\infty$ & $N$ & 0 & 0 & 0 & 0 & 0 & 0 & 0 & 0 & 0 \\
\hline$a$ & $a$ & $a$ & $r$ & $N$ & 0 & 0 & 0 & 0 & 0 & 0 & 0 & 0 & 0 \\
\hline$a$ & $a$ & $a$ & $r$ & $N$ & 0 & 0 & 0 & 0 & 0 & 0 & 0 & 0 & 0 \\
\hline$a$ & $a$ & $a$ & $\infty$ & $N$ & 0 & 0 & 0 & 0 & 0 & 0 & 0 & 0 & 0 \\
\hline$\infty$ & $\bar{\infty}$ & $\infty$ & $\stackrel{n}{r}$ & 요 & 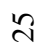 & ¿ి & $\supseteq$ & $\stackrel{\infty}{-}$ & 드 & $\stackrel{0}{-}$ & 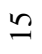 & $\Xi$ & 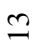 \\
\hline
\end{tabular}




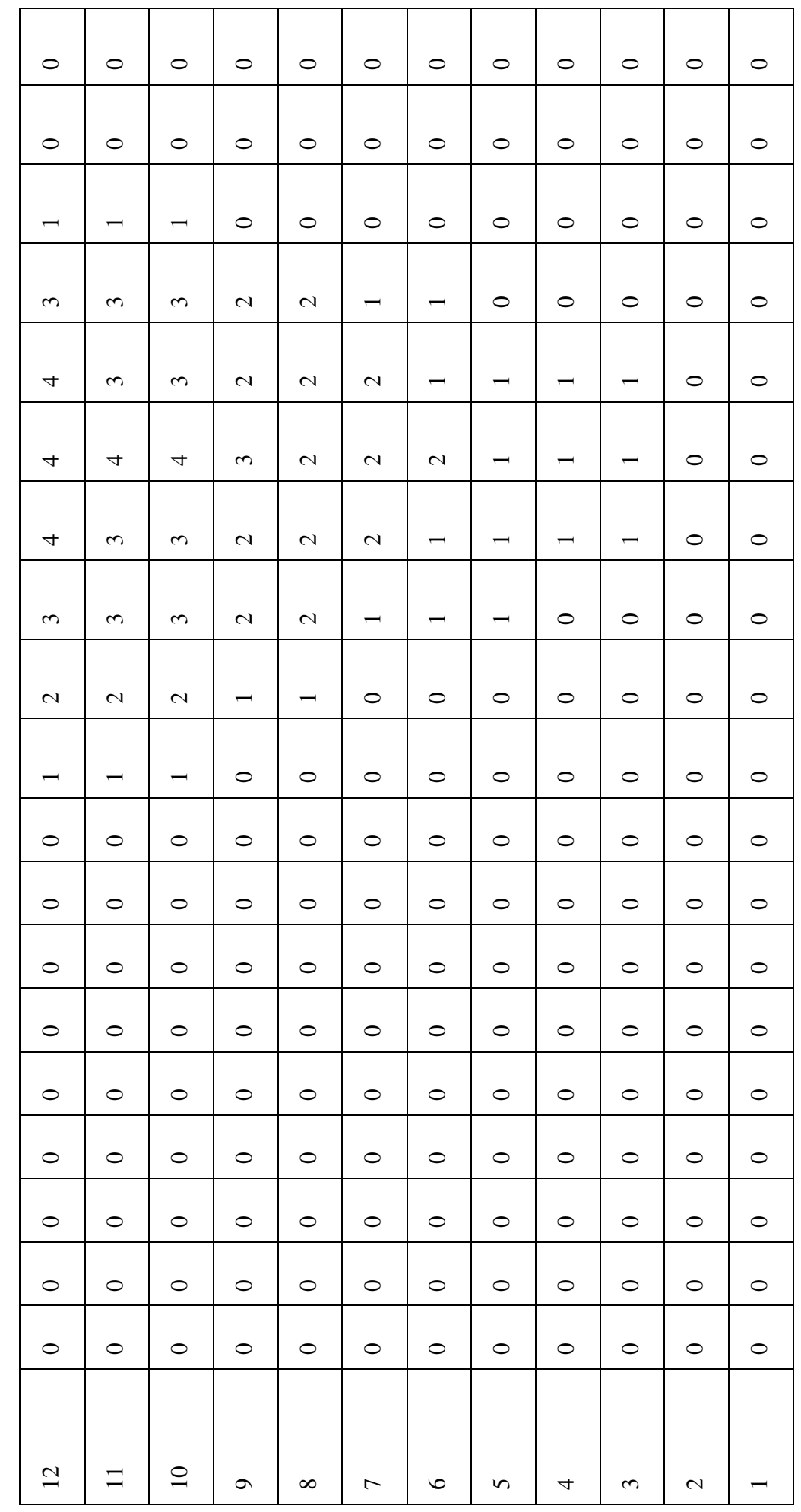

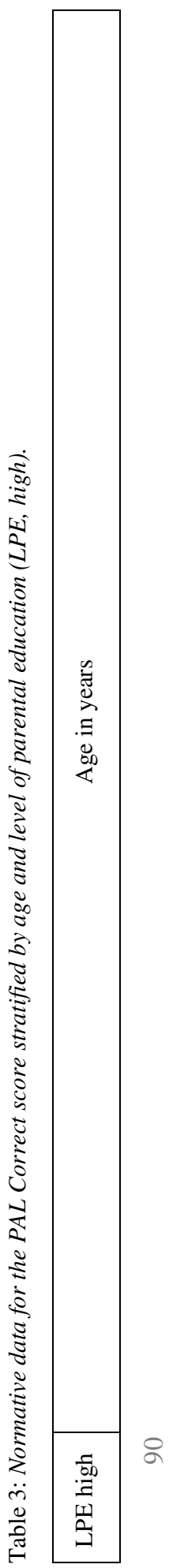




\begin{tabular}{|c|c|c|c|c|c|c|c|c|c|c|c|c|c|}
\hline$\stackrel{\because}{ \pm}$ & $\infty$ & I & I & $\underline{-}$ & $\underline{0}$ & $\stackrel{0}{0}$ & $\cong$ & $\cong$ & $\stackrel{n}{2}$ & $\cong$ & \pm & \pm & \pm \\
\hline$\stackrel{\circ}{ \pm}$ & ㄱ. & $\stackrel{2}{2}$ & $\stackrel{\infty}{=}$ & $\infty$ & I & I & I & $\underline{0}$ & $\underline{-}$ & $\stackrel{0}{0}$ & $\stackrel{0}{0}$ & $\stackrel{\bullet}{ }$ & $\cong$ \\
\hline$\stackrel{n}{n}$ & ণి & $\stackrel{2}{ }$ & $\stackrel{\infty}{=}$ & $\infty$ & I & ㄷ & I & I & $\underline{\ddots}$ & $\underline{\ddots}$ & $\underline{0}$ & $\stackrel{\bullet}{0}$ & $\cong$ \\
\hline$\stackrel{0}{\ddot{2}}$ & $\bar{\sim}$ & $\stackrel{\lambda}{ }$ & $\stackrel{2}{二}$ & $\stackrel{2}{2}$ & $\stackrel{\infty}{=}$ & 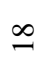 & $\stackrel{\infty}{-}$ & I & I & I & $I$ & $\underline{\ddots}$ & $\stackrel{0}{-}$ \\
\hline$\stackrel{\sim}{\simeq}$ & $\vec{\sim}$ & 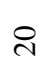 & 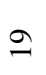 & $\stackrel{2}{ }$ & 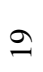 & 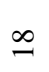 & $\stackrel{\infty}{\underline{0}}$ & 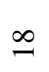 & I & I & I & I & ㄷ \\
\hline$\stackrel{\circ}{\stackrel{\Delta}{c}}$ & $\vec{\sim}$ & శ્ & 이 & $\stackrel{2}{=}$ & 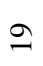 & $\stackrel{\infty}{=}$ & $\stackrel{\infty}{-}$ & $\stackrel{\infty}{=}$ & $\cong$ & I & I & I & ㄷ \\
\hline$\stackrel{n}{=}$ & $\bar{\sim}$ & ิㅗ & $\stackrel{2}{=}$ & $\stackrel{2}{ }$ & $\stackrel{\infty}{-}$ & $\stackrel{\infty}{=}$ & $\infty$ & $\infty$ & ㄷ & I & I & I & \\
\hline$\stackrel{\circ}{=}$ & $\bar{\sim}$ & ㄱ. & $\stackrel{2}{2}$ & $\infty$ & $\stackrel{\infty}{-}$ & 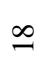 & I & ㄷ & ㅍ & 드 & $\underline{0}$ & $\underline{0}$ & 느 \\
\hline$\stackrel{n}{\varrho}$ & ત્તి & 9 & $\propto$ & $\infty$ & I & I & I & I & $\underline{\ddots}$ & $\underline{-}$ & $\underline{\ddots}$ & $\underline{\ddots}$ & 느 \\
\hline$\stackrel{\circ}{\circ}$ & 9 & $\stackrel{\infty}{-}$ & $\infty$ & I & I & $\underline{0}$ & $\underline{0}$ & $\underline{0}$ & $\underline{\ddots}$ & 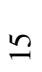 & $\cong$ & $\cong$ & 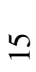 \\
\hline$\tilde{a}$ & $\stackrel{\infty}{-}$ & 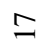 & 드 & $\mathscr{0}$ & 0 & $\stackrel{0}{ }$ & $\bumpeq$ & $\cong$ & 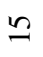 & 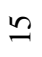 & \pm & \pm & \pm \\
\hline$\stackrel{\circ}{\circ}$ & $\stackrel{\infty}{-}$ & I & $\underline{0}$ & $\underline{0}$ & $\stackrel{n}{2}$ & $\stackrel{n}{n}$ & $\stackrel{n}{2}$ & $\Xi$ & $\Xi$ & $\Xi$ & $\Xi$ & $\stackrel{m}{=}$ & 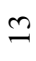 \\
\hline$n$ & I & $\underline{0}$ & $\stackrel{n}{2}$ & $\stackrel{n}{\sim}$ & $\Xi$ & $\Xi$ & $\Xi$ & $\Xi$ & $\stackrel{m}{-}$ & $\stackrel{m}{=}$ & $\stackrel{M}{=}$ & 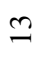 & 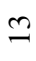 \\
\hline$\stackrel{\infty}{\infty}$ & 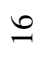 & $\stackrel{n}{2}$ & $\stackrel{n}{2}$ & \pm & $\Xi$ & 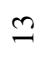 & $\stackrel{m}{=}$ & $\stackrel{m}{-}$ & $\stackrel{m}{=}$ & I & $\simeq$ & 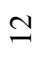 & $\simeq$ \\
\hline$\stackrel{n}{r}$ & $\mathscr{0}$ & 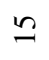 & $\Xi$ & \pm & $\stackrel{m}{=}$ & 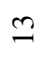 & $\stackrel{2}{=}$ & 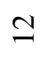 & 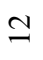 & $\simeq$ & $\simeq$ & 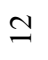 & $=$ \\
\hline$\stackrel{\circ}{\circ}$ & $\underline{0}$ & $\stackrel{n}{2}$ & $\Xi$ & $\stackrel{m}{=}$ & $\stackrel{m}{=}$ & $\stackrel{m}{=}$ & $\simeq$ & 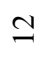 & $\simeq$ & I & $\simeq$ & $=$ & $=$ \\
\hline ?ִ & 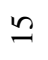 & $\Xi$ & \pm & $\stackrel{2}{2}$ & 9 & $\stackrel{m}{=}$ & $\simeq$ & 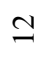 & 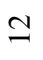 & $\simeq$ & $=$ & $=$ & $=$ \\
\hline$\stackrel{0}{0}$ & $\underline{-}$ & $\stackrel{n}{2}$ & $\Xi$ & $\stackrel{n}{=}$ & $\stackrel{2}{-}$ & $\stackrel{m}{=}$ & $\simeq$ & 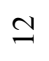 & 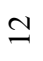 & $\simeq$ & $\simeq$ & $=$ & $=$ \\
\hline$\ddot{n}$ & $\stackrel{0}{ }$ & $\stackrel{n}{2}$ & $\stackrel{n}{2}$ & \pm & \pm & $\stackrel{m}{=}$ & $\underline{2}$ & 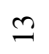 & $\stackrel{m}{2}$ & 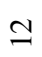 & $\simeq$ & $\simeq$ & $\simeq$ \\
\hline & ฉ̀ & a & $\hat{a}$ & ๙ & 2 & J & ๙ૂ & নু & $\bar{a}$ & $\sigma$ & $\infty$ & $\infty$ & $\infty$ \\
\hline
\end{tabular}




\begin{tabular}{|c|c|c|c|c|c|c|c|c|c|c|c|c|c|}
\hline \pm & \pm & 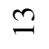 & 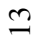 & $\cong$ & $\mathscr{2}$ & 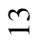 & $\simeq$ & 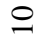 & $r$ & $r$ & $r$ & 0 & 0 \\
\hline$\because$ & 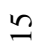 & $\simeq$ & $\because$ & $\because$ & \pm & \pm & \pm & $=$ & $a$ & $\infty$ & $\infty$ & $\infty$ & $\infty$ \\
\hline 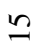 & 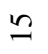 & 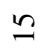 & 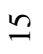 & 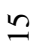 & 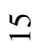 & \pm & \pm & $=$ & $a$ & $\infty$ & $\infty$ & $\infty$ & $\infty$ \\
\hline$\stackrel{0}{ }$ & $\stackrel{0}{-}$ & $\mathscr{0}$ & $\stackrel{0}{ }$ & 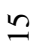 & 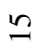 & $\because$ & 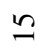 & $\simeq$ & 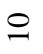 & $a$ & $a$ & $a$ & $a$ \\
\hline $\mathscr{0}$ & $\stackrel{0}{-}$ & $\mathscr{0}$ & $\stackrel{0}{ }$ & $\stackrel{0}{ }$ & $\stackrel{0}{ }$ & $\stackrel{0}{0}$ & 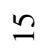 & 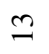 & 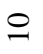 & $a$ & $a$ & $a$ & $a$ \\
\hline 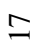 & $\stackrel{0}{-}$ & $\mathscr{0}$ & $\stackrel{0}{ }$ & $\stackrel{0}{ }$ & $\stackrel{0}{ }$ & $\stackrel{0}{0}$ & 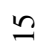 & 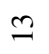 & & 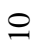 & $a$ & $a$ & $a$ \\
\hline $\mathscr{0}$ & 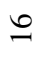 & 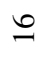 & $\mathscr{\sigma}$ & $\mathscr{\sigma}$ & $\stackrel{0}{\sigma}$ & 0 & $\cong$ & $\simeq$ & $\varrho$ & $a$ & $a$ & $a$ & $a$ \\
\hline 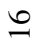 & $\varrho$ & $\underline{0}$ & $\underline{-}$ & $n$ & 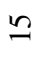 & $n$ & \pm & $\simeq$ & $ㅇ$ & $a$ & $a$ & $a$ & $a$ \\
\hline 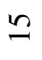 & $\cong$ & 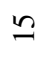 & 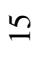 & 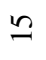 & 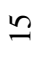 & $\curvearrowleft$ & \pm & $=$ & $a$ & $\infty$ & $\infty$ & $\infty$ & $\infty$ \\
\hline 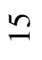 & 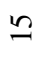 & $\Xi$ & $\Xi$ & \pm & $\Xi$ & \pm & 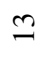 & $=$ & $\infty$ & $\infty$ & $\infty$ & $r$ & $r$ \\
\hline \pm & \pm & \pm & 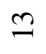 & 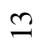 & 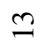 & 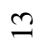 & $\simeq$ & 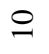 & $r$ & $r$ & $r$ & $r$ & 0 \\
\hline 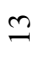 & $\cong$ & 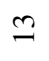 & 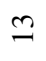 & $\stackrel{n}{-}$ & $\simeq$ & $\simeq$ & $\simeq$ & $a$ & $r$ & 0 & 0 & 0 & 0 \\
\hline$\simeq$ & $\simeq$ & $\simeq$ & $\simeq$ & $\simeq$ & $\simeq$ & $=$ & $=$ & $\infty$ & 6 & $n$ & $n$ & in & in \\
\hline$\simeq$ & $\simeq$ & $=$ & $=$ & $=$ & $=$ & $=$ & 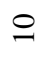 & $\infty$ & in & $n$ & $n$ & $\nabla$ & $\nabla$ \\
\hline$=$ & $=$ & $=$ & $=$ & $=$ & $\stackrel{ }{=}$ & $\stackrel{0}{=}$ & $\stackrel{\varrho}{0}$ & $r$ & in & $\nabla$ & $\nabla$ & $\nabla$ & $\nabla$ \\
\hline$=$ & $=$ & $=$ & $=$ & $\stackrel{0}{ }$ & 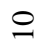 & 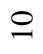 & 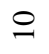 & $r$ & in & $\nabla$ & $\nabla$ & $\nabla$ & $\nabla$ \\
\hline$=$ & $=$ & 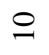 & 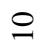 & $ㅇ$ & 으 & $ㅇ$ & $a$ & $r$ & $\nabla$ & $\nabla$ & $\nabla$ & $m$ & $m$ \\
\hline$=$ & $=$ & $=$ & $=$ & 으 & 으 & $ㅇ$ & 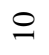 & $r$ & in & $\nabla$ & $\nabla$ & $\nabla$ & $\nabla$ \\
\hline$\simeq$ & $=$ & $=$ & $=$ & $=$ & $=$ & $\Xi$ & $\stackrel{\varrho}{ }$ & $\infty$ & in & $n$ & $\nabla$ & $\nabla$ & $\nabla$ \\
\hline$\infty$ & $\infty$ & 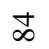 & $\infty$ & $\infty$ & $\bar{\infty}$ & $\triangleright$ & $\stackrel{n}{\sim}$ & in & $\stackrel{n}{\sim}$ & ते & $\stackrel{2}{2}$ & $\infty$ & ב \\
\hline
\end{tabular}




\begin{tabular}{|c|c|c|c|c|c|c|c|c|c|c|c|c|c|}
\hline 6 & 6 & 0 & 6 & 0 & $n$ & $n$ & $n$ & $n$ & $\forall$ & $\nabla$ & $\nabla$ & $m$ & $m$ \\
\hline$\infty$ & $r$ & $N$ & $\lambda$ & N & $r$ & $r$ & 6 & 0 & 0 & 0 & $n$ & $n$ & $\nabla$ \\
\hline$\infty$ & $\infty$ & $r$ & $r$ & $r$ & $r$ & $r$ & 6 & 0 & 6 & 6 & $n$ & $n$ & $\nabla$ \\
\hline$a$ & $\infty$ & $\infty$ & $\infty$ & $\infty$ & $\infty$ & $r$ & $r$ & $r$ & $r$ & $r$ & $r$ & 0 & 6 \\
\hline$a$ & $a$ & $a$ & $\infty$ & $\infty$ & $\infty$ & $\infty$ & $\infty$ & $r$ & $r$ & $r$ & $r$ & $r$ & 6 \\
\hline$a$ & $a$ & $a$ & $\infty$ & $\infty$ & $\infty$ & $\infty$ & $\infty$ & $r$ & $r$ & 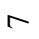 & $r$ & 6 & 6 \\
\hline$a$ & $a$ & $\infty$ & $\infty$ & $\infty$ & $\infty$ & $\infty$ & $\infty$ & $r$ & $r$ & $r$ & 0 & 0 & 0 \\
\hline$\infty$ & $\infty$ & $\infty$ & $\infty$ & $\infty$ & $\infty$ & $r$ & $r$ & $r$ & $r$ & 0 & 0 & $n$ & $n$ \\
\hline$\infty$ & $\infty$ & $r$ & $r$ & $r$ & $r$ & $r$ & $r$ & 0 & 0 & 0 & $n$ & $n$ & $\nabla$ \\
\hline$r$ & $r$ & $r$ & 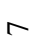 & 0 & 6 & 0 & 6 & $n$ & $n$ & $n$ & $\nabla$ & $\nabla$ & $m$ \\
\hline 0 & 6 & 0 & $n$ & $n$ & $n$ & $n$ & $n$ & $\nabla$ & $\forall$ & $\nabla$ & $\nabla$ & $m$ & $m$ \\
\hline 6 & $n$ & $n$ & $n$ & $n$ & $n$ & $\nabla$ & $\nabla$ & $\nabla$ & $\nabla$ & $m$ & $m$ & N & $N$ \\
\hline$n$ & $n$ & $\nabla$ & $\forall$ & $\nabla$ & $\nabla$ & $m$ & $m$ & $m$ & $m$ & $N$ & N & N & - \\
\hline$\nabla$ & $\nabla$ & $\nabla$ & $\forall$ & $m$ & $m$ & $m$ & $N$ & N & $N$ & $N$ & - & 一 & 0 \\
\hline$\nabla$ & $m$ & $m$ & $m$ & $m$ & $m$ & $N$ & $N$ & $N$ & - & - & - & 0 & 0 \\
\hline$m$ & $m$ & $m$ & $m$ & $m$ & N & N & $N$ & - & - & - & - & 0 & 0 \\
\hline$m$ & $m$ & $m$ & $m$ & $m$ & N & N & $N$ & N & - & - & - & 0 & 0 \\
\hline$m$ & $m$ & $m$ & $m$ & $m$ & $m$ & $N$ & $N$ & $N$ & $N$ & - & - & - & 0 \\
\hline$\nabla$ & $\nabla$ & $\nabla$ & $\nabla$ & $m$ & $m$ & $m$ & $n$ & $m$ & $N$ & $N$ & $N$ & 一 & - \\
\hline$\underline{-}$ & $\stackrel{n}{n}$ & $\Xi$ & $\cong$ & $\simeq$ & 二 & $\stackrel{ }{ }$ & $a$ & $\infty$ & N & 6 & $n$ & $\nabla$ & $m$ \\
\hline
\end{tabular}




\begin{tabular}{|c|c|c|c|c|c|c|c|c|c|c|c|c|c|}
\hline$N$ & - & \multirow{19}{*}{ 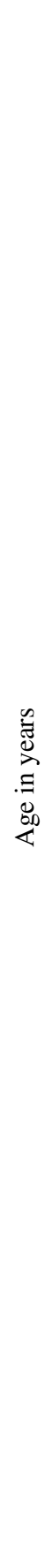 } & $\stackrel{n}{ \pm}$ & $\stackrel{0}{ }$ & 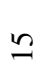 & 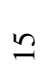 & $\Xi$ & \pm & $\Xi$ & $\cong$ & 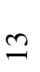 & 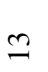 & $\cong$ \\
\hline$\nabla$ & $m$ & & $\stackrel{\circ}{\dot{I}}$ & $\stackrel{\infty}{-}$ & $\beth$ & $\mathscr{0}$ & $\stackrel{0}{ }$ & 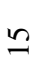 & 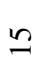 & 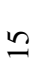 & \pm & \pm & \pm \\
\hline$\sigma$ & $m$ & & $\stackrel{n}{\sim}$ & $\stackrel{\infty}{-}$ & I & $\mathscr{0}$ & $\underline{0}$ & $n$ & $n$ & $n$ & $\because$ & さ & \pm \\
\hline$n$ & $\forall$ & & $\stackrel{\circ}{\ddot{2}}$ & $\cong$ & $\stackrel{\infty}{-}$ & I & I & 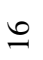 & $\stackrel{0}{ }$ & $\stackrel{0}{ }$ & $\because$ & $n$ & $\stackrel{n}{2}$ \\
\hline$n$ & $\forall$ & & $\stackrel{n}{i}$ & $\cong$ & $\infty$ & 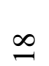 & I & 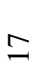 & 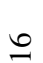 & $\mathscr{0}$ & 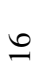 & 0 & $\simeq$ \\
\hline$n$ & $\nabla$ & & $\stackrel{\text { ㄱ. }}{\mathrm{I}}$ & $\stackrel{2}{ }$ & $\stackrel{\infty}{-}$ & $\infty$ & I & $\beth$ & $\stackrel{0}{-}$ & $\stackrel{0}{-}$ & $\underline{\sigma}$ & $\stackrel{0}{0}$ & 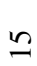 \\
\hline$n$ & $\forall$ & & $\stackrel{n}{=}$ & 9 & $\stackrel{\infty}{-}$ & I & I & $\beth$ & $\underline{0}$ & $\stackrel{0}{-}$ & $\stackrel{0}{-}$ & 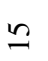 & 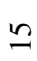 \\
\hline 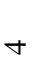 & $m$ & & $\stackrel{0}{=}$ & 9 & $\stackrel{\infty}{-}$ & I & $\stackrel{0}{0}$ & $\underline{0}$ & $\underline{0}$ & $n$ & $n$ & $n$ & $\simeq$ \\
\hline t & $m$ & & $\stackrel{n}{\varrho}$ & $\stackrel{\infty}{\sim}$ & I & $\mathscr{0}$ & $\underline{0}$ & $\underline{0}$ & $n$ & $n$ & $n$ & $\Xi$ & $\Xi$ \\
\hline$m$ & $N$ & & $\stackrel{\circ}{\circ}$ & ㄷ & $\stackrel{0}{0}$ & $\mathscr{0}$ & $\cong$ & 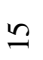 & \pm & \pm & \pm & $\Xi$ & $\cong$ \\
\hline$N$ & - & & $\tilde{a}$ & I & $\stackrel{\bullet}{ }$ & $\bumpeq$ & \pm & \pm & \pm & 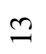 & 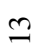 & 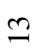 & $\cong$ \\
\hline- & 0 & & $\stackrel{\circ}{a}$ & $\underline{0}$ & $\cong$ & \pm & \pm & $\cong$ & $\stackrel{m}{=}$ & $\underline{\sim}$ & $\simeq$ & $\simeq$ & $\simeq$ \\
\hline 0 & 0 & & 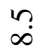 & $\cong$ & \pm & $\underline{n}$ & 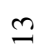 & $\cong$ & $\simeq$ & $\simeq$ & $\simeq$ & $=$ & $=$ \\
\hline 0 & 0 & & $\stackrel{\circ}{\infty}$ & \pm & 9 & 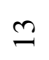 & $\simeq$ & $\simeq$ & 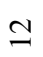 & $=$ & $=$ & $=$ & $=$ \\
\hline 0 & 0 & & $\stackrel{n}{r}$ & \pm & 9 & $\simeq$ & $\simeq$ & $=$ & $=$ & $=$ & 으 & 으 & 으 \\
\hline 0 & 0 & & $\stackrel{\circ}{r}$ & \pm & $\cong$ & $\simeq$ & $\simeq$ & $=$ & $=$ & $=$ & 으 & 으 & 으 \\
\hline 0 & 0 & & $\tilde{\sigma}$ & $\stackrel{m}{-}$ & $\simeq$ & $\simeq$ & $=$ & $=$ & $=$ & 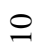 & 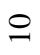 & 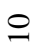 & 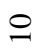 \\
\hline 0 & 0 & & $\stackrel{0}{0}$ & $\Xi$ & $\cong$ & $\simeq$ & $\simeq$ & $=$ & $=$ & $=$ & 으 & 으 & 으 \\
\hline 0 & 0 & & $n$ & $\Xi$ & $\cong$ & $\cong$ & $\simeq$ & $\simeq$ & $=$ & $=$ & $\Xi$ & 二 & 이 \\
\hline$N$ & - & $\frac{\frac{3}{0}}{\frac{11}{2}}$ & & ลे & $\stackrel{\infty}{\circ}$ & $\hat{a}$ & ๙ั & 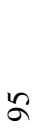 & d & $a$ & 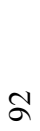 & $\bar{a}$ & ৪ \\
\hline
\end{tabular}




\begin{tabular}{|c|c|c|c|c|c|c|c|c|c|c|c|c|c|}
\hline$\simeq$ & $\simeq$ & 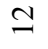 & 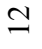 & $\simeq$ & $\simeq$ & $=$ & $=$ & $=$ & $=$ & $\stackrel{\varrho}{ }$ & $\infty$ & $n$ & $n$ \\
\hline \pm & \pm & $\stackrel{m}{-}$ & 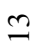 & 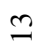 & $\stackrel{m}{-}$ & $\stackrel{m}{2}$ & 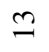 & $\cong$ & $\simeq$ & $\simeq$ & $a$ & $r$ & 6 \\
\hline \pm & \pm & \pm & $\stackrel{m}{-}$ & 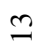 & $\stackrel{m}{-}$ & $\stackrel{m}{-}$ & $\stackrel{m}{-}$ & 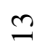 & $\stackrel{n}{-}$ & $\simeq$ & $a$ & $r$ & 6 \\
\hline 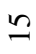 & 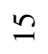 & \pm & \pm & \pm & \pm & $\Xi$ & \pm & 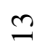 & $\stackrel{n}{=}$ & $\stackrel{m}{-}$ & 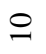 & $\infty$ & $r$ \\
\hline 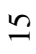 & $\bumpeq$ & 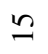 & 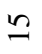 & \pm & \pm & $\Xi$ & \pm & \pm & \pm & 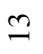 & $=$ & $\infty$ & $\infty$ \\
\hline 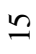 & 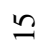 & 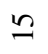 & 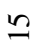 & \pm & \pm & $\Xi$ & \pm & \pm & \pm & 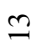 & $=$ & $\infty$ & $\infty$ \\
\hline 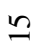 & 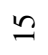 & 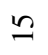 & \pm & $\Xi$ & $\Xi$ & $\Xi$ & \pm & \pm & \pm & 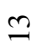 & $=$ & $\infty$ & $r$ \\
\hline 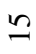 & \pm & \pm & \pm & \pm & $\Xi$ & $\Xi$ & $\underline{m}$ & 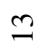 & $\stackrel{n}{-}$ & 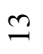 & 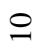 & $\infty$ & $r$ \\
\hline \pm & \pm & \pm & $\stackrel{m}{-}$ & 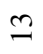 & $\stackrel{m}{-}$ & $\stackrel{m}{-}$ & 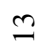 & 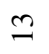 & $\stackrel{n}{-}$ & $\simeq$ & 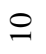 & $r$ & 6 \\
\hline$\cong$ & 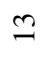 & $\cong$ & $\cong$ & $\stackrel{n}{=}$ & 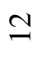 & $\simeq$ & 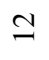 & $\simeq$ & $\simeq$ & $=$ & $a$ & 0 & 6 \\
\hline$\cong$ & $\simeq$ & I & I & $\simeq$ & $\simeq$ & $\simeq$ & $=$ & $=$ & $=$ & 이 & $\infty$ & 0 & in \\
\hline$\simeq$ & $\simeq$ & $=$ & $=$ & $=$ & $=$ & $=$ & $=$ & $\varrho$ & $\varrho$ & 으 & $r$ & $n$ & $\nabla$ \\
\hline$\Xi$ & $=$ & $=$ & $\varrho$ & $\stackrel{0}{=}$ & 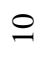 & 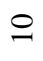 & $\stackrel{\varrho}{0}$ & 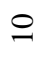 & 으 & $a$ & 0 & $\nabla$ & $m$ \\
\hline 으 & 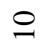 & 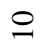 & $\stackrel{0}{ }$ & $\stackrel{0}{ }$ & $a$ & $a$ & $a$ & $a$ & $a$ & $\infty$ & 0 & $m$ & $m$ \\
\hline 으 & 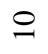 & $a$ & $a$ & $a$ & $a$ & $a$ & $a$ & $a$ & $\infty$ & $\infty$ & $n$ & $m$ & $N$ \\
\hline 으 & $a$ & $a$ & $a$ & $a$ & $a$ & $a$ & $\infty$ & $\infty$ & $\infty$ & $\infty$ & $n$ & $m$ & $N$ \\
\hline$a$ & $a$ & $a$ & $a$ & $a$ & $a$ & $\infty$ & $\infty$ & $\infty$ & $\infty$ & $r$ & $n$ & $N$ & $N$ \\
\hline 으 & $a$ & $a$ & $a$ & $a$ & $a$ & $a$ & $\infty$ & $\infty$ & $\infty$ & $\infty$ & $n$ & $m$ & $N$ \\
\hline 으 & $\stackrel{0}{ }$ & 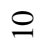 & $\stackrel{0}{ }$ & $\stackrel{ }{=}$ & $a$ & $a$ & $a$ & $a$ & $a$ & $\infty$ & 0 & $m$ & $m$ \\
\hline ळे & $\infty$ & $\hat{\infty}$ & $\infty$ & $\infty$ & ా & 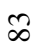 & శ్ & $\bar{\infty}$ & $\infty$ & $n$ & in & $\approx$ & तి \\
\hline
\end{tabular}




\begin{tabular}{|c|c|c|c|c|c|c|c|c|c|c|c|c|c|}
\hline$n$ & $n$ & $\nabla$ & $\nabla$ & $\nabla$ & $\nabla$ & $\nabla$ & $\forall$ & $m$ & $m$ & $m$ & $m$ & $m$ & $N$ \\
\hline 6 & 6 & 6 & 0 & 0 & $n$ & $n$ & $n$ & in & $n$ & $\nabla$ & $\forall$ & $\nabla$ & $\forall$ \\
\hline 6 & 6 & 6 & 0 & 0 & $n$ & $n$ & $n$ & $n$ & $n$ & $n$ & $\forall$ & $\nabla$ & $\forall$ \\
\hline$N$ & $N$ & $r$ & N & 0 & 6 & 0 & 6 & 6 & 6 & $n$ & $n$ & $n$ & $r$ \\
\hline$N$ & $r$ & $r$ & N & $N$ & $r$ & $N$ & 6 & 0 & 6 & 0 & $n$ & $n$ & $n$ \\
\hline$r$ & $r$ & $r$ & $N$ & $r$ & $r$ & $r$ & 0 & 6 & 6 & 0 & 6 & $n$ & $r$ \\
\hline$r$ & $r$ & $r$ & 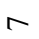 & $r$ & $r$ & 0 & 6 & 6 & 6 & 0 & $r$ & $r$ & $r$ \\
\hline$N$ & $r$ & $r$ & 6 & 0 & 6 & 0 & 6 & 6 & $r$ & 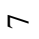 & $\lambda$ & N & 6 \\
\hline 0 & 6 & 0 & 6 & 0 & 0 & $n$ & $n$ & $n$ & $n$ & $n$ & $\forall$ & $\nabla$ & $\nabla$ \\
\hline 6 & $n$ & $n$ & $n$ & $n$ & $n$ & $n$ & $\nabla$ & $\forall$ & 6 & 0 & $n$ & $n$ & $n$ \\
\hline$n$ & $n$ & $n$ & $\forall$ & $\nabla$ & $\forall$ & $n$ & $n$ & $n$ & $n$ & $n$ & $\nabla$ & $\nabla$ & $\nabla$ \\
\hline$\nabla$ & $\nabla$ & $\nabla$ & $\nabla$ & $m$ & $m$ & $m$ & $m$ & $m$ & $m$ & $N$ & $N$ & N & $N$ \\
\hline$m$ & $m$ & $m$ & $m$ & $m$ & $m$ & $N$ & $N$ & $N$ & $m$ & $m$ & $m$ & $m$ & $N$ \\
\hline$m$ & $N$ & N & $N$ & N & N & $N$ & $N$ & $m$ & $m$ & $N$ & $N$ & N & $N$ \\
\hline$N$ & $\mathrm{~N}$ & N & N & N & - & - & - & - & $\mathrm{N}$ & N & N & 一 & - \\
\hline$N$ & $\mathrm{~N}$ & $N$ & $N$ & - & - & - & - & $N$ & 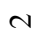 & N & 一 & - & - \\
\hline $\mathrm{N}$ & $\mathrm{N}$ & - & - & - & - & - & - & $N$ & $\mathrm{~N}$ & $\sim$ & $N$ & - & - \\
\hline$N$ & $N$ & $N$ & $N$ & - & - & - & - & - & $N$ & $N$ & $N$ & $N$ & - \\
\hline$m$ & $N$ & $N$ & $N$ & $N$ & $N$ & $N$ & 一 & - & - & $m$ & $n$ & $N$ & $N$ \\
\hline$\stackrel{2}{ }$ & $\infty$ & I & $\underline{-}$ & $\cong$ & $\Xi$ & 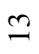 & $\simeq$ & $=$ & 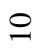 & $a$ & $\infty$ & $r$ & 6 \\
\hline
\end{tabular}




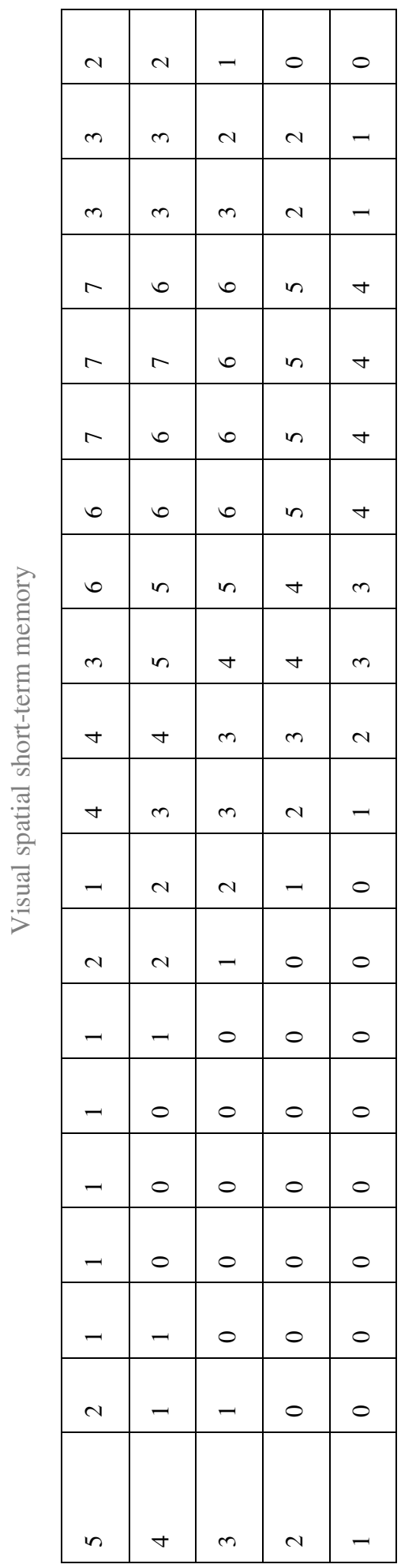

a 
苛 


\section{CHAPTER 4}

\section{Measuring goal setting in school-aged children: studying the influence of age, sex, and level of parental education and demographically corrected norms}

Toornstra, A., Hurks, P. P. M., Van der Elst, W., Kok, G., \& Curfs, L. M. G. (In preparation). Measuring goal setting in school-aged children: studying the influence of age, sex, and level of parental education and demographically corrected norms. 


\title{
Chapter 4
}

\begin{abstract}
Aim of the study was to establish demographically corrected norms for tasks measuring goal setting, and more specifically planning and reasoning in children. Three tasks were administered to $n=195$ Ukrainian children age 5.10 to 14.5 years old, i.e., the Spatial Working Memory (SWM), the Stockings of Cambridge test (SOC), and the Naglieri Nonverbal Ability test (NNAT). Main outcome per test was accuracy: i.e., the total number correct for the SOC and NNAT, and the total amount of incorrect responses for the SWM. Correlations among accuracy measures varied from -.51 to .60 indicating the measurement of related but simultaneously unique constructs. Age was associated with more accurate test performances on all outcome measures. On the NNAT we found a curvilinear association between age and accuracy, which means that younger children's NNAT accuracy score increased more with age compared to older children. A cubic age effect on accuracy was found for the SWM and SOC, i.e., test scores were relatively stable at younger and older ages, with a curvilinear increase in test scores in the other age groups. Sex was not associated with accuracy scores on any of the tests. Last, a higher level of parental education (LPE) was associated with higher accuracy scores, but only on the NNAT. Demographically corrected norms were presented per test. Demographic variables in norm analyses enhance insight in the scores and application in clinical settings and research.
\end{abstract}

Keywords: Visual spatial working memory, planning, problem solving, Spatial Working Memory, Stockings of Cambridge, $\mathrm{CANTAB}^{\odot}$, NNAT, continuous norming 


\section{Introduction}

Goal setting is an important skill for daily life. Anderson (2002, p. 74) defined goal setting as "the ability to develop new concepts, as well as the capacity to plan actions in advance and approach tasks in an efficient and strategic manner". Goal setting for example includes the ability to derive new notions (i.e., reasoning), plan steps to achieve end-goals and apply this plan efficiently. As such, deficits in goal setting may lead to inadequate planning, an inadequate execution of plans and limited conceptualizing of novel ideas while e.g., trying to solve a problem. The number of instruments available to measure goal setting in children is relatively scarce and suitable norm data are limited. However, interpretation of test scores is only meaningful when compared to suitable norm data for a sample of Ukrainian schoolchildren. Therefore, this study aims to collect norm data for three tests measuring goal setting: namely the Spatial Working Memory test (SWM) and the Stockings of Cambridge (SOC), which are both sub tests from the Cambridge Neuropsychological Test Automated Battery $\left(\right.$ CANTAB $^{\odot}$; Cambridge Cognition, 2012) and the Naglieri Nonverbal-reasoning Ability Test (NNAT; Naglieri, 2003), which are described in detail below. A relatively novel and promising method for norming is used here, which is called continuous norming (Van Breukelen \& Vlaeyen, 2005; Van der Elst et al., 2011). This method includes demographic variables of the sample to evaluate their influence on the scores and to increase the meaningfulness of the norms (Heaton et al., 2003). More information about this is provided below in the method section. These tests included, meaningful norming procedures and suitable statistical analyses are discussed next.

In the Spatial Working Memory (SWM) task, participants see boxes on the screen, and the task is to find as fast as possible tokens hidden behind some of the boxes (see Figure 1 for an example). A box can be opened by touching it. Applying a process of elimination enhances token search efficiency. As such, this task is believed to measure planning, i.e., how efficiently can one find the tokens.

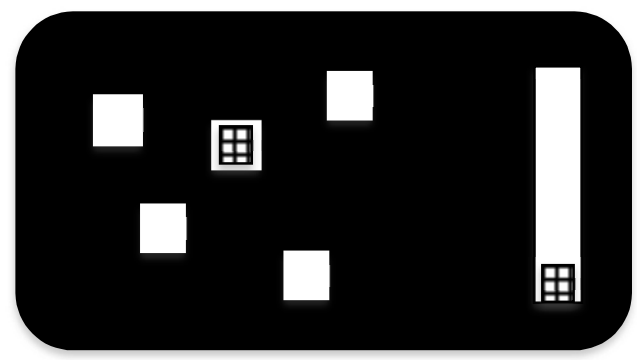

Figure 1. SWM on a touch screen: Simultaneous view of closed boxes, one open box with a token and the column on the right with a previously found token (this is not a real CANTAB ${ }^{\odot} \mathrm{SWM}$ sample). 


\section{Chapter 4}

The Stockings of Cambridge (SOC) is a computerized adaptation of the Tower of London task (Ozonoff et al., 2004; Shallice, 1982). The SOC measures the ability to plan and to execute a multi-step solution to a problem. Participants see colored circles ('balls') in the bottom half of the screen that need to be moved to 'stockings', until a pattern is created that is identical to the example pattern displayed in the top half of the screen. Moves can only be made according to a set of rules. One rule is for example, that a ball at the bottom cannot be moved if there is one on top of it (see Figure 2 for an example). The trials increase in level of difficulty by increasing the number of moves needed to solve a problem.

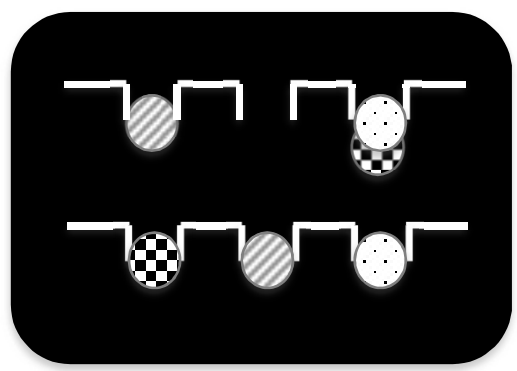

Figure 2: The SOC on a touch screen: A two-move problem. The balls in the bottom 'stockings' need to be dragged and moved till they are exactly in the same position as the top half of the screen (this is not a real $\mathrm{CANTAB}^{\odot} \mathrm{SOC}$ example).

Lastly, the Naglieri Nonverbal-reasoning Ability Test (NNAT) consists of pattern matrices. Participants need to identify the correct missing part from five response options shown in order to complete the target drawing (see Figure 3 for examples). This test is believed to measure reasoning, which falls within the functional domain of goal setting (Anderson, 2002; Giessman, Gambrell, \& Stebbins, 2013; Naglieri \& Ford, 2003).
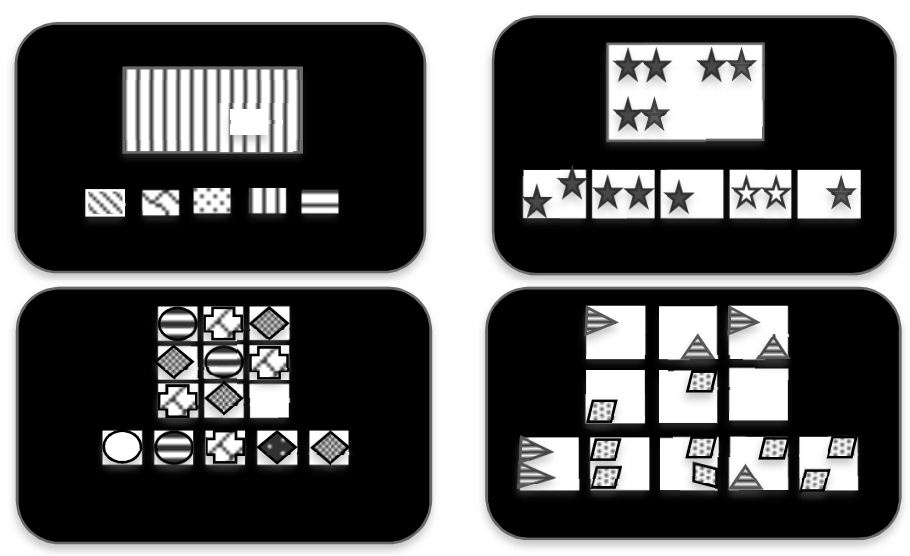

Figure 3. NNAT examples of the four different reasoning tasks: 1. Pattern completion, 2. Reasoning and analogy, 3. Serial reasoning, 4. Spatial visualization (these are not real NNAT samples). 
Our next aim is to collect norm data available for the tasks included in this study. In pediatric populations norm data available for the tests discussed here have, to the best of our knowledge, been calculated in a traditional way (De Luca et al., 2003; Lehto et al., 2003; Luciana \& Nelson, 2002; Roque et al., 2011). Calculations in the traditional way tend to be based on two-year age categories that are created in a sample. For example the norm for 7-8 years old children is based on the 7-8 year old subgroup in the sample only (Van der Elst et al., 2011). Furthermore, the boundaries between these subgroups raise points for discussion. A specific age sub group in the sample may have certain characteristics (i.e., performing unexpectedly well). These sampling characteristics for a specific age sub group would then become the norm for all children in that specific age bracket. The boundaries of subgroups themselves also raise concerns. Children of virtually the same age may be in different age groups, while children who differ almost two years may be in the same age category, i.e., a child of 12.9 is in the 11 to 12 years old age bracket while a child of 13.1 is in the 13 to 14 years old age group (Van der Elst et al., 2011). To avoid these discussion points in traditional norming a favorable method called continuous norming has been introduced, which will be applied in this study to collect SWM, SOC, and NNAT normative data for schoolchildren (Van Breukelen \& Vlaeyen, 2005; Van der Elst et al., 2011).

This distinctive method of norming is based on multiple regressions including several demographic variables, such as age, sex, and level of parental education (LPE). The demographic variables of age, sex, and LPE have been linked to differences in neuropsychological test performance in the literature (Lange, Chelune, Taylor, Woodward, \& Heaton, 2006; Strauss et al., 2006; Tallberg, Ivachova, Jones Tinghag, \& Östberg, 2008). In pediatric populations, research showed support for the validity of the SWM, SOC and NNAT, e.g., in children with ADHD (Fried, Hirshfield-Becker, Petty, Batchelder, \& Biederman, 2015), children with fetal alcohol spectrum disorders (Rasmussen, Soleimani, \& Pei, 2011) and in multi-lingual development in children (Naglieri, Booth, \& Winsler, 2004). Furthermore, studies involving typically developing children using the SWM, the SOC and the NNAT have shown that these tests can be administered to children from 5 years old onward (Luciana \& Nelson, 2002; Green et al., 2019; Carman \& Taylor, 2010; Naglieri \& Ronning, 2000). An age related development has been associated e.g., with improved goal setting abilities from 4 - 12 years old (Green et al., 2019; Luciana \& Nelson, 2002; Roque et al., 2011). However, the amount of studies examining the validity of goal setting tasks such as the SWM and the SOC in pediatric populations is still limited.

Data on the next demographic variable sex, are still inconclusive in goal setting abilities in children. For instance, Pangelinan et al. (2011) did not find sex differences on the SWM in their sample of 172 children aged 6-13 years old. Rojahn and Naglieri (2006) did not find sex differences on the NNAT test in children from 6-9 years old. In 10-13 years old children however, females scored slightly higher on this test and there was a slight sex difference in favor of males in 15-16 years old children. Rojahn and Naglieri (2006) deemed the effects too small to apply to norm scores. 


\section{Chapter 4}

Additionally, the demographic variable level of parental education (LPE) has limitedly been studied in relation to goal setting abilities in schoolchildren. LPE is perceived as an estimate of socio-economic status (SES, White, 1982). It has been found to correlate positively with children's higher academic achievement (Hackman \& Farah, 2009) and cognitive functions other than goal setting, e.g., working memory (Mezzacappa, 2004; Naglieri \& Ronning, 2000a; Noble et al., 2007). These findings underline the relevance to study the influence of age, sex, and LPE on the goal setting tasks here and to include demographic variables in these analyses attempting to establish norms.

In the statistical method of regression (Van der Elst et al., 2011) sex and LPE are categorical variables and age is a continuous variable and can simultaneously be included. This means that age as a continuous variable will be applied to each data point in the sample (Bechger et al., 2009). Both the option of including several demographic variables in the analyses and the application of each variable to all data (and not a specific sub category in the data) makes these regressions more adequate and individualized (Van der Elst et al., 2011). Last, the sample under study differs from the norm populations of the tests in this study. The current sample consists of schoolchildren in Ukraine. Non-verbal tests have been thought to be suitable for measurement in different populations but may not be culture free (e.g., in their design) thus supporting our thesis (Rosselli \& Ardila, 2003). Norms have to the best of our knowledge not been established for Ukraine and are therefore needed in order to interpret scores in a meaningful manner (Lange et al., 2006). The aim of the present study was to assess the effect of demographic variables and establish a normative data range on the SWM, SOC and NNAT test, for a sample of $n=195$ schoolchildren in Ukraine, age 5.10 to 14.5 years old.

\section{Methods}

\section{Participants}

The sample of $n=195$ children was enrolled in local schools in Ukraine, which provide a nine-year compulsory education up till the children are in general 15 years old (Table 1). Age was approximately evenly distributed except for the youngest and oldest age groups. Per school all children were invited and $80.5 \%$ participated. The level of parental education (LPE) was based on the level of education completed by the parent(s) in a household; low described education up till grade 9 (similar to primary and middle school) and high applied to grade 10 (high school or polytechnic) and above, according to the Ukrainian educational system (Ukraine Channel, 2017). Education is compulsory up till and including grade 9. The highest level was assigned as the mean for the household (high vs. low) in the $6.5 \%$ of the cases showing a discrepancy between parent (or caregiver)'s level of education (in with e.g., Koball \& Jiang, 2018). 
Table 1: Basic Demographic Data

\begin{tabular}{|c|c|c|c|c|c|c|c|}
\hline & \multirow[t]{2}{*}{$\mathrm{n}$} & \multirow[t]{2}{*}{$\begin{array}{l}\text { Age (Mean } \\
\text { in years) }\end{array}$} & \multirow[t]{2}{*}{ Age SD } & \multicolumn{2}{|l|}{ Sex } & \multicolumn{2}{|c|}{$\begin{array}{l}\text { Level of } \\
\text { Parental } \\
\text { Education } \\
\text { (LPE) }\end{array}$} \\
\hline & & & & Female & Male & Low & High \\
\hline SWM & 169 & 9.89 & 2.43 & 78 & 91 & 17 & 152 \\
\hline SOC & 152 & 10.02 & 2.43 & 69 & 83 & 14 & 138 \\
\hline NNAT & 195 & 9.74 & 2.52 & 95 & 100 & 24 & 171 \\
\hline
\end{tabular}

\section{Procedure and instrument}

Researchers contacted schools to partake in the study, after which responding school managements invited parents to information meetings about this study. Information and consent letters were provided by the researchers and the school management and then taken home. Testing started after parents and children had given informed consent letters to their class teachers.

The SWM and SOC, as part of the Cambridge Neuropsychological Test Automated Battery (CANTAB ${ }^{\odot}$, Cambridge Cognition, 2012), and the NNAT were administered individually in a separate room. $\mathrm{CANTAB}^{\odot}$ trained testers administered the SOC and SWM on a HP Pavilion TS sleek-book laptop with a 15-inch touch screen. The NNAT was administered on a different day within the same week with the provided trial booklet. Two trained researchers administered tests. Instructions followed the pre-described scripts and practice items in the school's language of instruction. All tests terminated after their respective termination rules (see materials). The research ethics committee of the Faculty of Psychology and Neuroscience of Maastricht University, The Netherlands, approved this study. All data were obtained in compliance with ethics regulations of the WMA declaration of Helsinki (2013). School management received debriefing by anonymous group reports per class.

\section{Materials}

First, Spatial Working Memory (SWM) is a computerized self-ordered search task, which requires planning and cognitive flexibility (Cambridge Cognition, 2012). Validity for the SWM in children was found among others in clinical studies on early childhood deprivation (Bos, Fox, Zeanah, \& Nelson, 2009; Sachse et al., 2013). Both studies 


\section{Chapter 4}

compared the clinical groups to control groups and found that $\mathrm{CANTAB}^{\odot}$ test findings distinguished cognitions between both groups in line with expectations related to previously made diagnoses. Lowe and Rabbitt (1998) found a moderate reliability (0.6-0.68) on the $\mathrm{CANTAB}^{\odot} \mathrm{SWM}^{\text {SW }}$

In this task, blue tokens need to be found in boxes that appear on the screen. Once a token has been found, this token is placed in an empty column on the right side of the screen (Figure 1). Tokens are hidden according to pre-set rules unknown to the participant: The box where a token was found will not have a token again for the duration of this particular trial. Once a token has been found, a continued search from that box working in a systematic way is deemed most effective (Owen, Downes, Sahakian, Polkey, \& Robbins, 1990). Each trial starts with a similar screen with boxes and the trials increase in difficulty by increasing the number of tokens that need to be found to a maximum of 8 (thus increasing the chance of errors as more boxes need to be remembered that contained a token). Main outcome measure is the number of errors, i.e., fewer errors are thought to reflect an efficient search strategy and are therefore seen as a measure of accuracy (Cambridge Cognition, 2012). The SWM Total Errors, is used for norming. The break off point for the whole test is based on too many total errors. Total administration time is on average 10 minutes.

The Stocking of Cambridge (SOC; Cambridge Cognition, 2012) is a computerized task of planning and reasoning. Validity for the SOC in children among others was found in a clinical study on autism spectrum disorders and controls in 6 to 47 years old (Ozonoff et al., 2004). Lowe and Rabbitt (1998) found a moderate reliability (0.6-0.68) on an older version of the $\mathrm{CANTAB}^{\odot} \mathrm{SOC}$. The SOC test procedure is as follows: Two displays with three colored balls each are shown on a screen. The balls in the lower half of the screen need to be moved until they form an exact copy of the example pattern in the upper part on the screen (see Figure 2). The balls can only be moved according to specific rules, e.g., only one ball can be moved at a time, and a ball at the bottom cannot be moved if there is one on top. The number of moves is seen as a measure of the ability to plan, with fewer moves indicating better planning ability. The problems increase in difficulty with respect to the necessary minimum number of moves, from 2 to 5 moves, to copy the image at the top of the screen. Each of these trials starts with a new screen. The outcome measure 'Problems solved in minimum moves' reflects the amount of times a trial was successfully completed, using the minimum amount of moves possible. The best possible score is 12 (i.e., 12 problems solved using the minimum number of moves). The test break off point is when three consecutive problems registered as too many moves (the exact amount is dependent on the problem, i.e., 7 moves for a problem that can be solved in 3 moves). Total administration time is on average 13 minutes.

Last, the Naglieri Nonverbal Ability Test (NNAT) is an untimed one-on-one task on paper (Naglieri, 2003). It purports to measure reasoning and goal setting. The test consists of four different forms of reasoning in increasingly difficult trials and thus involves cognitive flexibility and goal setting (see Figure 3). Proof of the test's validity for children was found in correlations of the group-version of the NNAT with well-established tests, 
such as the Raven's Progressive Matrices (RPM) test (Naglieri, 2003; Naglieri \& Ronning, 2000b; Raven, 2000). The procedure is as follows: One page shows a two-colored matrix with a part left blank. The correct missing part needs to be chosen from 5 response options underneath (see Figure 3). The outcome measure is the summation of correct responses (maximum possible is 72). Total administration time of this untimed paper test depends on the individual student; the break off point is four consecutive incorrect trials.

\section{Statistical analyses}

First means and standard deviations were calculated for accuracy scores of all tests (see Table 2). Then the observed means of the Ukrainian schoolchildren were compared to the observed means of the Western norm samples for the SWM, the SOC and the NNAT respectively by evaluating the overlap between the confidence intervals (CIs) of the two samples. This comparison was done based on the Western age sub groups, and sub groups that were the same compared to the Ukrainian sample, which means the oldest and youngest children were excluded. Next Pearson zero order correlations were calculated between the Ukrainian SWM, SOC, and NNAT observed scores (Table 3). Fractional polynomial (regression) models were established for each outcome measure of the SWM, SOC and NNAT, with the demographic variables age, sex, level of parental education (LPE) and their two-way interactions (e.g., age * LPE). Age was centered (Age_C = calendar age in months - mean age of the sample 116.44 months), and quadratic (age_C ${ }^{2}$ ) and cubic (age_C $\mathrm{C}^{3}$ ) age were included to evaluate curvilinear effects, i.e., scores increase less with age in older children (Van der Elst et al., 2011). Fractional polynomial models with exponents were used to achieve a better fit of the plotted observed scores and the predicted scores including the same demographic variables (age, sex, LPE) and their twoway interactions (Van der Elst et al., 2016). A restricted set of 7 exponents $\{-2,-1,-0.5,0$, $0.5,1,2\}$ is used. Sex was dummy coded as $0=$ female and $1=$ male. LPE was dummy coded as $0=$ low and $1=$ high. We established which demographic variables were predictive for each of the outcomes measures of the SWM, SOC, and NNAT. The full models were reduced in a stepwise hierarchical way by removing the least significant predictor if its $p$-value was higher than 0.05 and Akaike's Information Criterion was used to identify the two best powers ( AIC; i.e., the lower the better the fit) for the models.

Next, the assumptions of fractional polynomial (regression) analysis were tested for each model, that is the normal distribution of residuals, the absence of heteroscedasticity, multicollinearity and influential cases (Field, 2009; Van der Elst et al., 2006). The normal distribution was checked using the Kolmogorov-Smirnov test. The occurrence of multicollinearity was tested by calculating the Variance Inflation Factors $(\mathrm{VIF} \leq 10)$ while Cook's distance and standard deviation of residuals $>|3|$ were used to identify influential cases (Fisher et al., 2014). The residuals were plotted and visually inspected. Last, the standardized residuals were grouped in quartiles based on the predicted scores. Levene's test was used to evaluate heteroscedasticity in the standardized residuals based on these quartile groups. 


\section{Chapter 4}

In the last step, the normative data were calculated using the expected scores for each participant based on the final models. The residuals were calculated (= observed score - expected score). The residuals were standardized using the standard deviation $(S D)$ of the residuals of the analyzed sample (= residual /SD (residual) of the normative sample; Van der Elst et al., 2011). Last, the residuals were converted in percentile values based on the normal distribution if the normality assumption (as evaluated in the Kolmogorov-Smirnov test) is met. If this normality assumption is not met, the empirical distribution of the standardized residuals of the sample is used. Making these calculations per child in practice is tedious so tables with these converted scores are in the appendix to support ease of use. An alpha level of .05 was applied to avoid Type 1 errors. All calculations were made in SPSS version 24 and $R$.

\section{Results}

The means and standard deviations $(S D)$ for the SWM, the SOC and the NNAT outcome measures (observed untransformed scores of the Ukrainian sample) are presented in Table 2. The tests were part of a test battery and especially younger children started to drop out in the latter part of this battery consisting of the SWM and the SOC (i.e., not starting the test), while others dropped out due to absenteeism and/or illness (SWM $n=169$ and SOC $n=152$ children).

Table 2. Means and standard deviations (SD) for the SWM, the SOC and the NNAT outcome measures (observed scores)

\begin{tabular}{|l|l|l|l|l|l|}
\hline & Mean & $\begin{array}{l}\text { Std. } \\
\text { Deviation }\end{array}$ & $\mathrm{n}$ & $\begin{array}{l}\text { Minimum of } \\
\text { the sample }\end{array}$ & $\begin{array}{l}\text { Maximum of } \\
\text { the sample }\end{array}$ \\
\hline SWM Total Errors & 54.65 & 21.30 & 169 & 2 & 96 \\
\hline $\begin{array}{l}\text { SOC Problems Solved } \\
\text { Minimum Moves }\end{array}$ & 5.08 & 3.09 & 152 & 0 & 11 \\
\hline NNAT & 19.85 & 8.93 & 195 & 0 & 48 \\
\hline
\end{tabular}

The results of the correlation analysis between the SWM, SOC and NNAT outcome measures are presented in Table 3. All accuracy measures correlated significantly with each other in the expected direction, meaning that higher accuracy on one is linked to higher accuracy on the other (i.e., lower is better on SWM errors hence the negative correlations with NNAT and SOC problems solved in minimum moves scores). 
Table 3: Pearson zero order correlations for the SWM, the SOC and the NNAT outcome measures and the demographic variables

\begin{tabular}{|c|c|c|c|c|c|c|c|}
\hline & & 1 & 2 & 3 & 4 & 5 & 6 \\
\hline 1 & Age & 1 & & & & & \\
\hline 2 & Sex & 0.03 & 1 & & & & \\
\hline 3 & $\begin{array}{l}\text { Level of Parental Education } \\
\text { (LPE) }\end{array}$ & 0.13 & -0.1 & 1 & & & \\
\hline 4 & SWM Total Errors & $-.47 * *$ & 0.0 & $-.18 *$ & 1 & & \\
\hline 5 & $\begin{array}{l}\text { SOC Problems Solved } \\
\text { Minimum Moves }\end{array}$ & $.40^{* *}$ & -0.2 & $.21^{*}$ & $-.53 * *$ & 1 & \\
\hline 6 & NNAT & $.51 * *$ & 0.0 & $.35 * *$ & $-.51 * *$ & $.60 * *$ & 1 \\
\hline
\end{tabular}

** CoRRELATION IS SIGNIFICANT AT THE 0.01 LEVEL (2-TAILED).

* Correlation is SignificAnt AT THE 0.05 LEVEL (2-TAiLED).

Next, the final regression models $(p<.00)$ are shown in Table 4 . The NNAT, the SWM, and the SOC are all curvilinear models. The AICs are for the SOC 618.11 and the SWM 1509.36. Box-Cox transformations were applied to SWM Total Error and SOC scores, because preliminary analyses suggested heteroscedasticity with the untransformed scores (Osborne, 2010). Also, outliers, which are scores with an absolute standardized residual $S D>3.0$ were removed. After these transformations and removal of outliers, the assumptions of regression analyses were mostly met for the final models, i.e., KolmogorovSmirnov values $p \geq .05$, except for SWM Total errors $p=.000$. All values of the Levene's statistic $p \geq .05$; Cook's distance values $\leq .01$; and all Variance Inflation Factors $\leq 1.02$ (except for SWM Total Errors, VIF 6.4, which is below the cut-off of 10 that is indicative of multi-collinearity). There were only significant main effects and no significant interaction effects. The SWM Total Errors did not meet the normality assumption as evaluated in the Kolmogorov-Smirnov test. Calculations for this analysis were therefore not based on the standard normal distribution but on the distribution of the standardized residuals (Zhou, 1998).

None of the participants obtained the maximum or lowest possible score (lowest, i.e., 0 errors on the SWM) on any of the outcome measures. The best score achieved were 11 (out of 12) on the SOC by $2 \%, 2$ errors on the SWM by $0.6 \%$, and 48 (out of 72) on the NNAT by $0.5 \%$ of the participants. Last, the confidence intervals (CIs, $p=0.01$ ) for the grand mean scores showed overlap for the SWM (CI Ukrainian sample ranges between 


\section{Chapter 4}

40.82 - 56.92 and 18.96 - 47.99 for the CANTAB norm group; Cambridge Cognition, 2014). The CIs of the grand mean scores showed minimal overlap between the Ukrainian sample and the CANTAB norm group on the SOC (CI 3.99-6.55 and 6.06-8.75 respectively). There was no overlap between the CIs of the grand mean NNAT scores (CI Ukrainian children $15.69-21.31$ and CI $22.80-30.45$ in the NNAT norm sample; Naglieri, 2003), so the score ranges differed between the two samples.

Table 4: Final regression models for the SWM, the SOC and the NNAT

\begin{tabular}{|c|c|c|c|c|c|c|c|}
\hline Test score & $\mathrm{n}$ & Variable & B & $\mathrm{p}$ & $\mathrm{t}$ & $\begin{array}{l}\text { Stand. } \\
\text { deviation } \\
\text { (residual) }\end{array}$ & $\mathrm{R}^{2}$ \\
\hline \multirow{4}{*}{$\begin{array}{l}\text { SWM Total Errors } \\
\text { (Box-Cox } \\
\text { transformation, } \\
\lambda 1.40 \text { ) }\end{array}$} & \multirow{2}{*}{$\begin{array}{l}16 \\
9\end{array}$} & (Constant) & 121.04 & .000 & 0.63 & & \\
\hline & & $\mathrm{Age}^{2}$ & 0.17 & .000 & -1.59 & & \\
\hline & & $\mathrm{Age}^{3}$ & -0.0069 & .000 & 1.63 & & \\
\hline & & $\begin{array}{l}\mathrm{Age}^{3} \\
\text { (LNAge) }\end{array}$ & 0.001 & .000 & 2.36 & 47.27 & $.27 *$ \\
\hline \multirow{3}{*}{$\begin{array}{l}\text { SOC Problems Solved } \\
\text { in Minimum Moves } \\
\text { (Box-Cox } \\
\text { transformation, } \lambda 1.60 \text { ) }\end{array}$} & $\begin{array}{l}15 \\
2\end{array}$ & (Constant) & -2.57 & .000 & -0.86 & & \\
\hline & & $\mathrm{Age}^{3}$ & 0.00009 & .000 & 3.64 & & \\
\hline & & $\begin{array}{l}\mathrm{Age}^{3} \\
\text { (LNAge) }\end{array}$ & -0.00002 & .000 & -3.54 & 4.20 & $.21 *$ \\
\hline \multirow[t]{4}{*}{ NNAT } & $\begin{array}{l}19 \\
3 * \\
*\end{array}$ & (Constant) & 14.96 & .000 & 9.99 & & \\
\hline & & Age_C & 0.16 & .000 & 9.43 & & \\
\hline & & Age_C ${ }^{2}$ & -0.002 & .002 & -3.19 & & \\
\hline & & LPE & 7.53 & .000 & 5.13 & 6.61 & $.41 *$ \\
\hline
\end{tabular}

$* p<.000$

** $N$ is lower due to removal of outliers 


\section{Normative procedure}

Based on the established regression models, norms for the SWM, the SOC and the NNAT outcome measures are calculated for the Ukrainian sample by applying the procedure described above (norm tables in appendices). Box-Cox transformations were reverted back to untransformed scores (Osborne, 2010). For example, presume that an 11 years old child completed 20 items correct on the NNAT and level of education of the child's parents is high. The regression model for the NNAT is Constant $+\mathrm{B}_{1}$ (Age_C) + $\mathrm{B}_{2}\left(\right.$ Age_C $\left.\mathrm{C}^{2}\right)+\mathrm{B}_{3}(\mathrm{LPE})$ with the model presented in Table 4 . This is Constant $+0.16 *$ (age child in months - average age sample $)+\left(-0.002 *\right.$ Age_C $\left.C^{2}\right)+(1 * 7.53)$, that is $14.96+$ $[0.16 *(132-116.44)]+(-0.002 * 242.11)+7.53=24.5$. The residual is computed, which is $20-24.5=-4.5$. In the third step the residual is standardized $-0.68(-4.5 / 6.61)$. The standardized residual is turned into a percentile value based on the standard normal cumulative distribution. A standardized residual of -0.68 corresponds with a percentile value equal to 23 . This means that $23 \%$ of the population of 11 years old children with high LPE obtain a NNAT score that is equal or lower than 20. The NNAT score of this child is therefore in the lower part of the normal range $\left(5^{\text {th }}-95^{\text {th }}\right.$ percentile). Lastly, the NNAT outcome measure showed quadratic age effect, which indicates a curvilinear development with age. The SWM and SOC scores showed cubic age effects, that is a trajectory with multiple curves. The cross-sectional developmental trajectories of all scores are in Figure 4. The NNAT and SOC show in principle increasing accuracy between the ages of 5.5 to 13.5 years old and then start to level off. Figure 4 also shows development of scores with age for the outcome measure SWM Total Errors in which a lower score is better. Several bends in the regression line are typical for a cubic age effect as in SWM Total Errors, which appears to stabilize from 13 years old. Norm tables are in the appendix. 


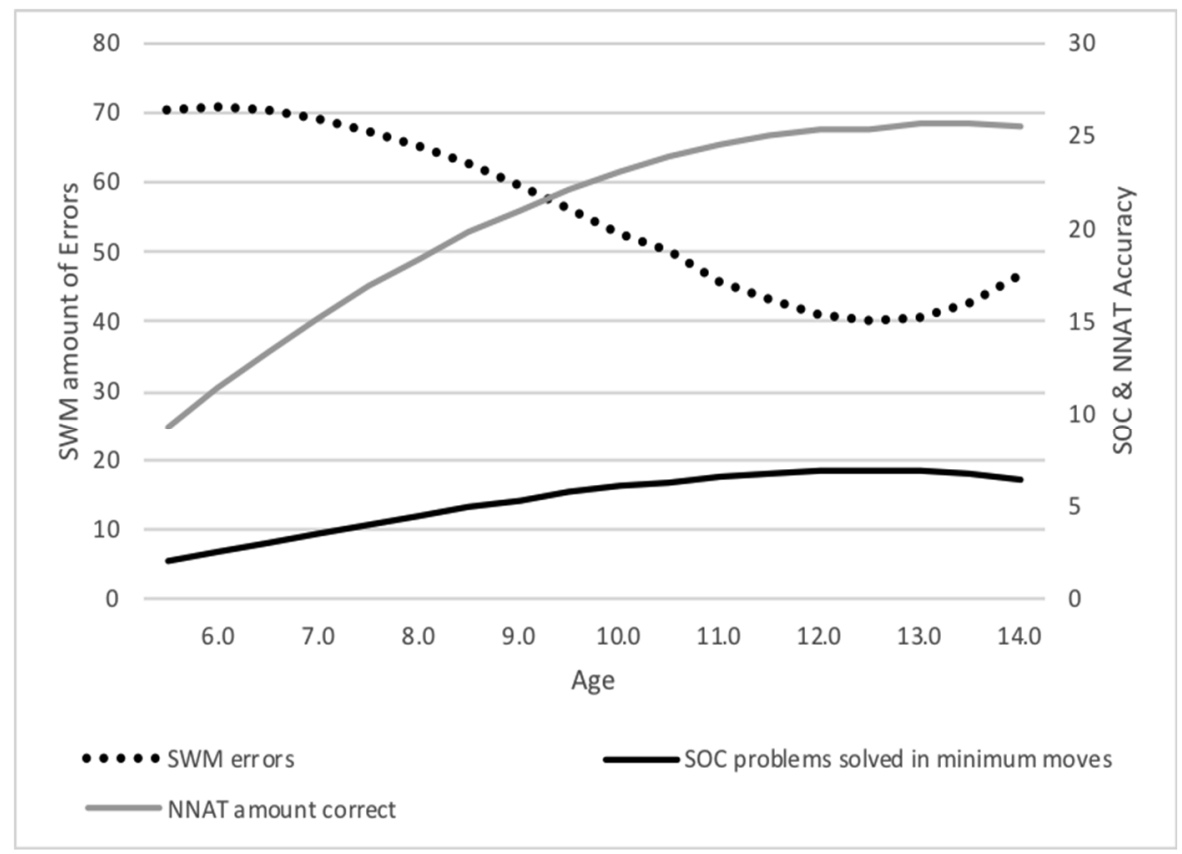

Figure 4. Trajectory of the regression-based scores for the SWM, the SOC, the NNAT (high LPE) accuracy scores by age

\section{Discussion}

The aim of this study was to establish demographically corrected pediatric norms for three tests measuring goal setting, and more specifically planning and reasoning in school aged children in the Ukraine. Anderson (2002) defined goal setting as "the ability to develop new concepts, as well as the capacity to plan actions in advance and approach tasks in an efficient and strategic manner" (p. 74). The normal range of performance on these tasks was established by applying an auspicious method called continuous norming. This different approach to norming included taking into consideration the impact of continuous and categorical demographic variables simultaneously on test performance. Demographic variables included in this study are, age, sex, and level of parental education (LPE; Van Breukelen \& Vlaeyen, 2005; Van der Elst et al., 2011). This method results in more suitable estimates of norm scores for reasons considered in the introduction. Results of this study will be discussed next.

First, we found that accuracy scores on these tests correlated in the expected directions. These correlations support the notion that planning, reasoning and executing the planned steps to achieve a goal occur in the domain of EFs as hypothesized by Anderson (2002). The correlations are on the other hand moderate (i.e., strong > .90), which indicates differences in underlying constructs in the tests. 
Second, we found age-related changes on test performance on all tests, i.e., we found that on average older children made fewer errors on the SWM and solved higher amounts of problems on the SOC and the NNAT. Pangelinan et al. (2011) associated age in typically developing children from $6-12.9$ years old to increasing accuracy on the SWM. Furthermore, the NNAT showed in principle curvilinear development with age, and stabilizing scores in the oldest children. The SOC and the SWM showed cubic development with age, i.e., some stabilizing in scores at lower and higher ages (Figure 4). Development of working memory, a key component underlying planning as required in the SWM, SOC and NNAT, has been associated with (curvi)linear development with age from early childhood (Anderson, 2002; De Luca et al., 2003; Gathercole et al., 2004; Luciana \& Nelson, 200; Naglieri, 2003). However, Crescentini, Seyed-Allaei, Vallesi, and Shallice (2012) showed that planning also differs from memory (i.e., areas of brain activation involved) in a Tower of London task (comparable to the SOC). This is in line with Anderson (2002) who posed that planning and goal setting are related executive functions, yet different from other functions such as information processing. Therefore, in principle increased accuracy found with age may be associated with planning and goal setting which was also shown in the relatively fewer studies on normative data in children on the SWM, SOC (Green et al., 2019; DeLuca et al., 2009; Lehto et al., 2003; Luciana \& Nelson, 2002; Roque et al., 2011).

Third, the Ukrainian sample was compared to the Western norm to examine whether test scores overlapped (Rosselli \& Ardila, 2003). The comparison was limited to traditional age sub groups, which was the only available demographic variable for both populations (Cambridge Cognition, 2014; Naglieri, 2003). The confidence intervals (CIs) of the grand mean of the SWM between the Ukrainian sample and the CANTAB norm sample overlapped, which was also but only just the case for the SOC scores. This indicates a comparable performance in planning, and goal setting (Lehto et al., 2003). The NNAT however, showed no overlap in CIs between the Ukrainian sample compared to the Western mean scores. Additionally, $0.5 \%$ in the Ukrainian sample achieved the highest score on the NNAT, which was 48 for the sample (out of a maximum possible of 72 on this test) so there were no test ceiling effects. Therefore, the NNAT scores appear to indicate a lower mean compared to the Western population. However, these comparisons were only based on descriptive data (CIs) and did not include the demographic variables sex and LPE, which would need to be included in future statistically more rigorous comparisons assessing cultural differences in test scores.

Fourth, we found neither sex effects, nor an age * sex interaction on any of the tests. This is in line with Luciana \& Nelson (1998) who did not find sex differences on the accuracy scores of the SOC and SWM. Sex differences were found in a study on a large sample of the NNAT, but Rojahn and Naglieri (2006) deemed effect sizes negligible and were subsequently not included in the standardized norms, supporting the findings here.

The last finding is an effect of the demographic variable of level of parental education (LPE) on the NNAT, linking higher NNAT accuracy to higher LPE. LPE is associated with socio-economic status of families, which in turn is linked to life-outcomes 


\section{Chapter 4}

such as cognitive development of children (Bradley \& Corwyn, 2002). Bronfenbrenner and Morris (2006) hypothesized that planning is an example of a cognitive ability that is subject to environmental influences i.e., a child that experiences modelling and learning of planning from e.g., the adults in his/her life may be more likely to use it when a situation requires. Lohman and Gambrell (2012) however, found that children of lower SES performed better on the NNAT. Lohman and Gambrell (2012) link these results to test design differences, i.e., describing the NNAT and the Raven's as figural reasoning tests as opposed to most other non-verbal reasoning tests. The latter are thought to use among others, pictures and line drawings which may be associated with other constructs in testing (e.g., language).

There are some limitations in interpreting the data of this study. First, the number of parents with a low LPE group was smaller than the high level LPE group. In multiple linear regressions each data point of the whole sample is used (i.e., relation of the demographic variables to the dependent variable of the test score) and thus fewer data are needed to arrive at statistically valid results (Van Breukelen \& Vlaeyen, 2005). Secondly, the number of regressions computed might increase the chance of type I errors. Although it cannot be stated with absolute certainty that some relationships are not chance findings (Piechatzek et al., 2009), the discussion shows that the findings in our study are supported by (neuro)psychological literature. Furthermore, discussing the difference between shortterm memory and working memory is not within the scope of this study (Gluck, Mercado, $\&$ Meyers, 2008, p. 171-179). Discussions here focus on the ability of keeping information in memory for a short while and to manipulate that information according to task demands, which aligns with Anderson's conceptualization of working memory (2002). The third limitation concerns e.g., Carman and Taylor's questions about the validity of methods related to the NNAT and its ability to identify gifted children from different SES backgrounds (2010).

\section{Conclusion}

Demographically corrected norms were established for three tests measuring goal setting in children, i.e., the SWM, the SOC, and the NNAT. Demographic variables (age, sex, LPE) were associated with test scores on these tests, but not all were significant. Including demographic variables is therefore important when calculating norm scores (Mitrushina et al., 2005). Two tests showed differences in score trajectories compared to the Western norms (i.e., cubic age effects), which supports our contention to examine differences between populations in relation to test scores (Rosselli \& Ardila, 2003). Providing the norm tables and establishing the normal range for different populations especially for functions that are difficult to evaluate (e.g., the executive functions of planning, reasoning and goal setting) is important for use in clinical and research settings. 


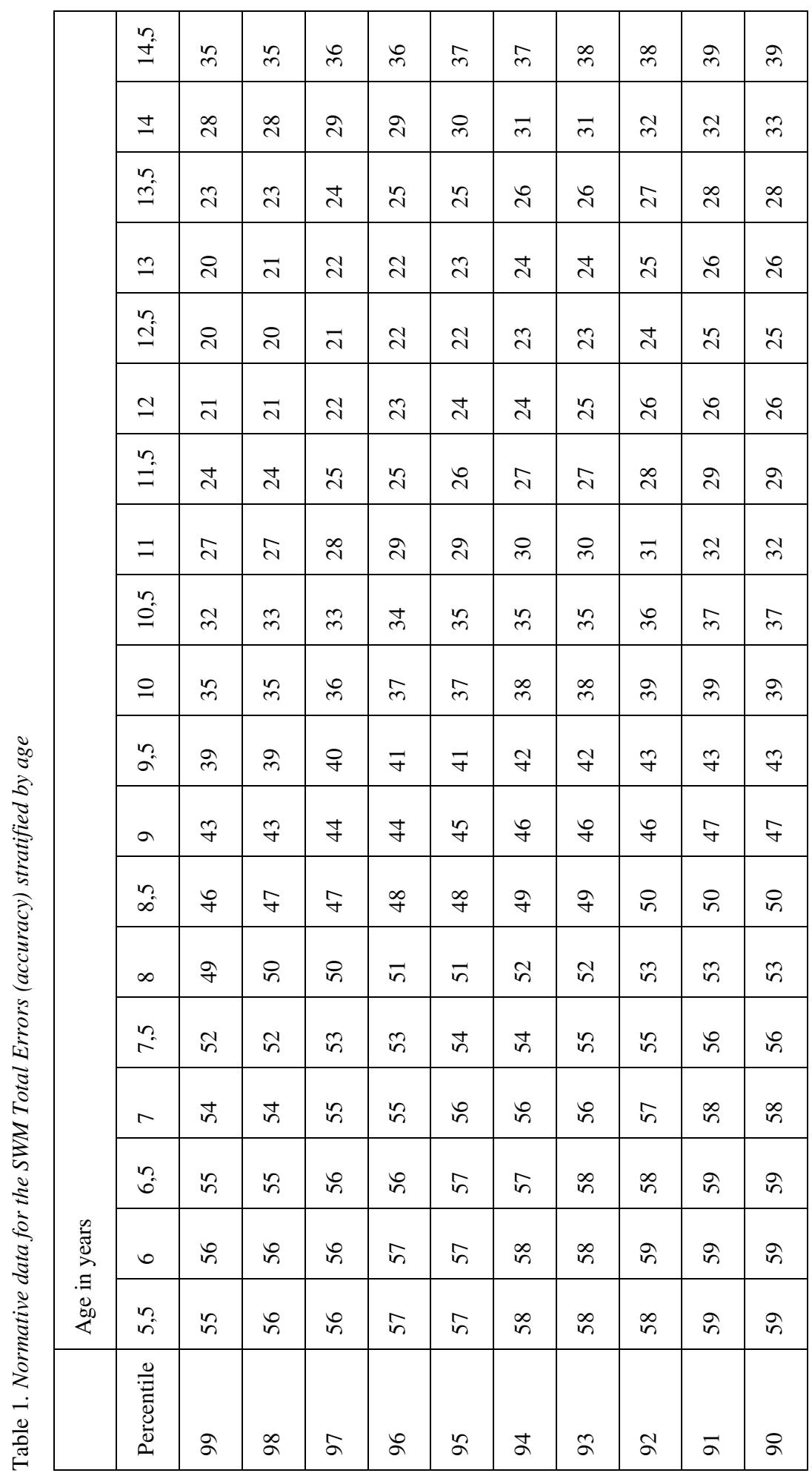




\begin{tabular}{|c|c|c|c|c|c|c|c|c|c|c|c|c|c|}
\hline ले & q & q & q & q & $F$ & $F$ & $\bar{F}$ & F & F & $\stackrel{?}{f}$ & กิ & 8 & $\widetilde{\sigma}$ \\
\hline$\tilde{m}$ & $m$ & $m$ & $\vec{m}$ & $\dot{m}$ & $\stackrel{+}{m}$ & $\check{m}$ & $\ddot{m}$ & $\ddot{n}$ & 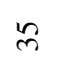 & $\hat{m}$ & fo & $i n$ & $i$ \\
\hline$\stackrel{\infty}{\sim}$ & ¿े & તે & নे & i & i & $\bar{m}$ & $\bar{m}$ & $\bar{m}$ & $\bar{m}$ & $m$ & $q$ & $\vec{n}$ & $\hat{n}$ \\
\hline$\stackrel{\sim}{\sim}$ & $\stackrel{i}{\circ}$ & $\bar{\lambda}$ & $\hat{\lambda}$ & $\hat{\lambda}$ & $\stackrel{\sim}{\sim}$ & $\stackrel{i}{i}$ & $\stackrel{\infty}{\sim}$ & ঐे & ঐे & $\bar{m}$ & $F$ & in & $\vec{n}$ \\
\hline$\stackrel{\sim}{\sim}$ & $\stackrel{i}{i}$ & i & $\hat{\lambda}$ & $\hat{\lambda}$ & $\hat{\lambda}$ & $\stackrel{\infty}{\sim}$ & $\stackrel{\infty}{\sim}$ & $\stackrel{\infty}{\sim}$ & $\stackrel{\infty}{\sim}$ & i & 우 & $\stackrel{q}{q}$ & $\vec{n}$ \\
\hline $\bar{\lambda}$ & $\hat{\imath}$ & $\hat{\lambda}$ & $\stackrel{\infty}{\sim}$ & $\stackrel{\infty}{\sim}$ & $\stackrel{\infty}{\sim}$ & ते & ते & ฉે & in & $\bar{m}$ & $F$ & in & $\tilde{n}$ \\
\hline ते & 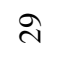 & $\stackrel{m}{\text { r }}$ & i & 尺 & $\bar{m}$ & $\bar{m}$ & $\bar{m}$ & 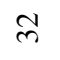 & $\approx$ & ले & $\mathscr{q}$ & $\tilde{n}$ & $\tilde{n}$ \\
\hline$\approx$ & ले & $m$ & m & ले & mे & mे & mे & $\ddot{m}$ & $\ddot{m}$ & $\hat{m}$ & f & $n$ & $\stackrel{\circ}{\circ}$ \\
\hline$\hat{m}$ & $\hat{m}$ & $\stackrel{\infty}{m}$ & $\underset{m}{\infty}$ & $\underset{\infty}{\infty}$ & ले & ले & ले & \& & P & $F$ & in & in & 8 \\
\hline q & q & q & 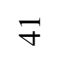 & $\bar{\gamma}$ & $F$ & F & F & F & F & 拐 & $\tilde{n}$ & $\bar{\sigma}$ & $\widehat{\sigma}$ \\
\hline 打 & $\forall$ & F & 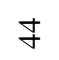 & $\stackrel{f}{f}$ & $\stackrel{f}{f}$ & iq & fo & $\mathscr{q}$ & $\mathscr{q}$ & $\stackrel{\infty}{+}$ & $i$ & ț & 6 \\
\hline F & $\stackrel{\infty}{+}$ & $\stackrel{\infty}{+}$ & $\stackrel{\infty}{+}$ & $\stackrel{\infty}{+}$ & $\stackrel{q}{q}$ & qे & gे & in & in & $\bar{n}$ & in & $\hat{\sigma}$ & 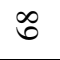 \\
\hline $\bar{n}$ & $\bar{n}$ & $\bar{n}$ & $\bar{n}$ & $\approx$ & $\approx$ & $\tilde{n}$ & $n$ & $n$ & $n$ & in & $\mathcal{\sigma}$ & $\stackrel{R}{R}$ & $\nabla$ \\
\hline in & $\ddot{n}$ & in & $\ddot{n}$ & $n$ & $i n$ & $n$ & $n$ & $i$ & $i$ & in & 6 & $\approx$ & $\stackrel{\nabla}{\sim}$ \\
\hline in & in & in & $i n$ & $\hat{n}$ & $\hat{n}$ & $\stackrel{\infty}{n}$ & $\stackrel{\infty}{n}$ & $\stackrel{\infty}{n}$ & $\stackrel{\infty}{n}$ & 8 & $\widehat{\sigma}$ & $\stackrel{n}{\sim}$ & $\stackrel{\circ}{\sim}$ \\
\hline$\stackrel{\infty}{n}$ & $\stackrel{\infty}{n}$ & $\stackrel{\infty}{n}$ & in & in & in & in & 8 & 8 & 8 & $\bar{\sigma}$ & 8 & $\approx$ & $\stackrel{\infty}{\sim}$ \\
\hline in & in & in & 8 & 8 & 8 & $\vec{\sigma}$ & $\bar{\sigma}$ & $\bar{\sigma}$ & $\bar{\sigma}$ & $\widehat{్}$ & 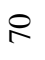 & $\stackrel{\infty}{\sim}$ & $\stackrel{2}{2}$ \\
\hline in & 8 & 8 & 8 & 8 & $\bar{\sigma}$ & $\overrightarrow{6}$ & $\bar{\sigma}$ & $\mathcal{b}$ & $\widetilde{\sigma}$ & $\tilde{6}$ & $\nabla$ & $\stackrel{\infty}{\sim}$ & 2 \\
\hline in & in & 8 & 8 & 8 & 8 & $\overrightarrow{6}$ & $\overline{6}$ & $\bar{\sigma}$ & $\bar{\sigma}$ & 8 & $尺$ & $\stackrel{\infty}{\sim}$ & $\stackrel{2}{r}$ \\
\hline ळे & $\infty$ & $\infty$ & $\infty$ & $\infty$ & ఉ & $\infty$ & $\tilde{\infty}$ & $\bar{\infty}$ & $\infty$ & $\stackrel{2}{\sim}$ & in & $\stackrel{2}{a}$ & ते \\
\hline
\end{tabular}




\begin{tabular}{|c|c|c|c|c|c|c|c|c|c|c|c|c|c|}
\hline 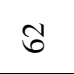 & $\widetilde{\sigma}$ & 8 & 8 & 8 & 8 & t & t & t & t & $\ddot{8}$ & 8 & $\ddot{8}$ & 8 \\
\hline$i$ & in & $\stackrel{\infty}{n}$ & $\stackrel{\infty}{n}$ & $\infty$ & $\stackrel{\infty}{n}$ & $\stackrel{\infty}{n}$ & $\stackrel{\infty}{n}$ & in & in & in & 8 & 8 & 8 \\
\hline$\tilde{n}$ & it & in & in & in & in & $i n$ & in & $i n$ & in & $\ddot{n}$ & $i$ & in & in \\
\hline$\vec{n}$ & $\therefore$ & $\approx$ & $\approx$ & $n$ & $n$ & $n$ & in & $\tilde{n}$ & $n$ & 䓟 & in & in & in \\
\hline $\bar{n}$ & $\bar{n}$ & $\approx$ & $\approx$ & $\approx$ & $\approx$ & $n$ & $n$ & $\approx$ & $n$ & 吉 & $\stackrel{\sharp}{n}$ & 吾 & in \\
\hline$\approx$ & $\approx$ & $n$ & in & in & $n$ & in & 䓟 & 苚 & in & in & $i$ & in & in \\
\hline$n$ & 点 & in & $i n$ & $n$ & $i n$ & $i n$ & in & $\stackrel{\circ}{n}$ & $\stackrel{i}{n}$ & in & in & in & in \\
\hline$i$ & in & in & in & in & in & $\stackrel{\infty}{n}$ & $\stackrel{\infty}{n}$ & $\stackrel{\infty}{n}$ & in & in & in & in & 8 \\
\hline 8 & $\bar{\sigma}$ & $\bar{\sigma}$ & $\vec{b}$ & $\bar{\sigma}$ & $\widetilde{\sigma}$ & $\widetilde{\sigma}$ & $\widetilde{\sigma}$ & $\widehat{\sigma}$ & $\widetilde{\delta}$ & 8 & 8 & 8 & t \\
\hline రి & 8 & 8 & 8 & 8 & t & t & t & t & t & $\ddot{8}$ & $n$ & $\stackrel{8}{0}$ & 8 \\
\hline 8 & $\stackrel{8}{0}$ & 8 & 5 & 5 & 5 & 5 & 5 & $\tilde{\sigma}$ & $\stackrel{\infty}{\circ}$ & $\ddot{\circ}$ & $\stackrel{\infty}{\circ}$ & वे & b \\
\hline 8 & 8 & $R$ & $R$ & $尺$ & $\therefore$ & $\therefore$ & $\therefore$ & $\approx$ & $F$ & $F$ & $\vec{r}$ & $N$ & a \\
\hline 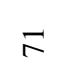 & $\therefore$ & $\approx$ & $\approx$ & $\approx$ & $\approx$ & $\approx$ & $\approx$ & $\approx$ & \pm & I & $\underset{ }{ \pm}$ & $\approx$ & $\approx$ \\
\hline I & $\approx$ & $\approx$ & 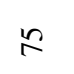 & $\approx$ & $\approx$ & 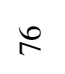 & $\therefore$ & $\stackrel{2}{2}$ & 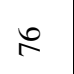 & \& & $\therefore$ & 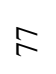 & 尺 \\
\hline$\stackrel{2}{\circ}$ & \& & 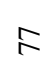 & 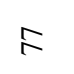 & $\therefore$ & $\stackrel{\infty}{\curvearrowright}$ & 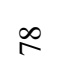 & 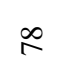 & $\stackrel{\infty}{\curvearrowright}$ & $\stackrel{\infty}{\curvearrowright}$ & R & $\stackrel{\imath}{ }$ & $\stackrel{\imath}{ }$ & 尺 \\
\hline$\stackrel{\infty}{\sim}$ & $\stackrel{\infty}{\curvearrowright}$ & 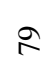 & $\stackrel{2}{2}$ & $\stackrel{\imath}{ }$ & g & g & $\infty$ & $\infty$ & $\infty$ & 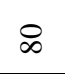 & $\vec{\infty}$ & $\vec{\infty}$ & $\vec{\infty}$ \\
\hline$\stackrel{\imath}{ }$ & g & $\infty$ & $\triangleright$ & $\infty$ & $\infty$ & $\vec{\infty}$ & $\bar{\infty}$ & $\bar{\infty}$ & $\bar{\infty}$ & $\infty$ & $\tilde{\infty}$ & $\tilde{\infty}$ & $\tilde{\infty}$ \\
\hline$\hat{\imath}$ & $\infty$ & $\infty$ & $\infty$ & $\infty$ & $\bar{\infty}$ & $\vec{\infty}$ & $\bar{\infty}$ & $\bar{\infty}$ & $\bar{\infty}$ & $\tilde{\infty}$ & $\tilde{\infty}$ & $\tilde{\infty}$ & $\infty$ \\
\hline$\stackrel{R}{ }$ & 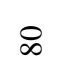 & $\infty$ & $\triangleright$ & $\infty$ & $\infty$ & $\vec{\infty}$ & $\vec{\infty}$ & $\vec{\infty}$ & $\vec{\infty}$ & $\tilde{\infty}$ & $\tilde{\infty}$ & $\widetilde{\infty}$ & $\widetilde{\infty}$ \\
\hline 2 & $\stackrel{\infty}{\simeq}$ & $=$ & $\underline{\varrho}$ & $\cong$ & \pm & 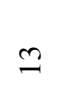 & $\simeq$ & $=$ & 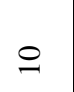 & $a$ & $\infty$ & $r$ & 0 \\
\hline
\end{tabular}




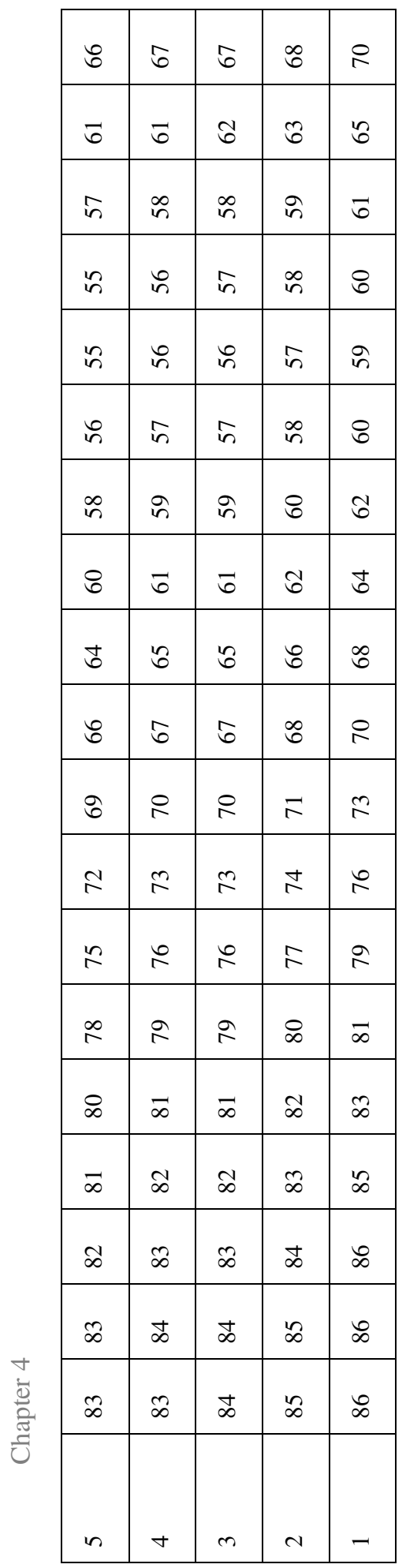

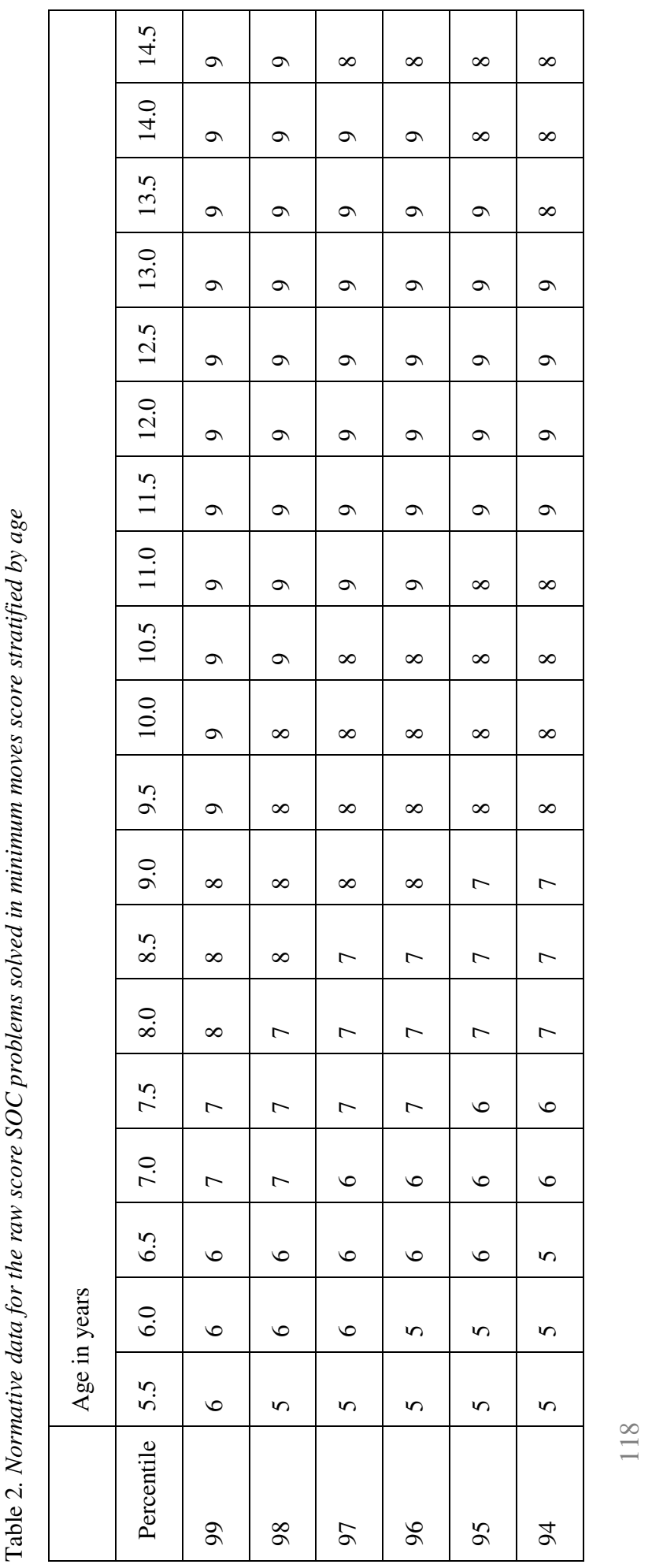




\begin{tabular}{|c|c|c|c|c|c|c|c|c|c|c|c|c|c|}
\hline$\infty$ & $\infty$ & $\infty$ & $\infty$ & $\infty$ & $\infty$ & $\infty$ & $N$ & 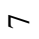 & $N$ & $r$ & $r$ & 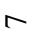 & $N$ \\
\hline$\infty$ & $\infty$ & $\infty$ & $\infty$ & $\infty$ & $\infty$ & $\infty$ & $\infty$ & $\infty$ & $\infty$ & $\infty$ & $\infty$ & $\infty$ & $N$ \\
\hline$\infty$ & $\infty$ & $\infty$ & $\infty$ & $\infty$ & $\infty$ & $\infty$ & $\infty$ & $\infty$ & $\infty$ & $\infty$ & $\infty$ & $\infty$ & $\infty$ \\
\hline$a$ & $\infty$ & $\infty$ & $\infty$ & $\infty$ & $\infty$ & $\infty$ & $\infty$ & $\infty$ & $\infty$ & $\infty$ & $\infty$ & $\infty$ & $\infty$ \\
\hline$a$ & $\infty$ & $\infty$ & $\infty$ & $\infty$ & $\infty$ & $\infty$ & $\infty$ & $\infty$ & $\infty$ & $\infty$ & $\infty$ & $\infty$ & $\infty$ \\
\hline$a$ & $\infty$ & $\infty$ & $\infty$ & $\infty$ & $\infty$ & $\infty$ & $\infty$ & $\infty$ & $\infty$ & $\infty$ & $\infty$ & $\infty$ & $\infty$ \\
\hline$\infty$ & $\infty$ & $\infty$ & $\infty$ & $\infty$ & $\infty$ & $\infty$ & $\infty$ & $\infty$ & $\infty$ & $\infty$ & $\infty$ & $\infty$ & $\infty$ \\
\hline$\infty$ & $\infty$ & $\infty$ & $\infty$ & $\infty$ & $\infty$ & $\infty$ & $\infty$ & $\infty$ & $\infty$ & $\infty$ & $\infty$ & $\infty$ & $\infty$ \\
\hline$\infty$ & $\infty$ & $\infty$ & $\infty$ & $\infty$ & $\infty$ & $\infty$ & $\infty$ & $r$ & $r$ & $r$ & $r$ & $r$ & $r$ \\
\hline$\infty$ & $\infty$ & $\infty$ & $\infty$ & $\infty$ & $r$ & $r$ & $r$ & $r$ & $r$ & $r$ & $r$ & 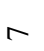 & $r$ \\
\hline$\infty$ & $r$ & $r$ & $r$ & $N$ & $N$ & $r$ & $r$ & N & $r$ & $r$ & $r$ & $r$ & $r$ \\
\hline$r$ & $r$ & $r$ & $r$ & 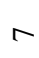 & $r$ & $r$ & $r$ & $r$ & $r$ & $r$ & $r$ & $r$ & 6 \\
\hline$r$ & $r$ & $r$ & $r$ & $N$ & $\sigma$ & 6 & 0 & 0 & 6 & 6 & 0 & 6 & 0 \\
\hline$r$ & 6 & 6 & 0 & 0 & 6 & 6 & 0 & 0 & 6 & 6 & 6 & 6 & 0 \\
\hline 6 & 0 & 6 & 6 & 6 & 6 & 6 & 0 & 6 & 6 & $n$ & $n$ & $n$ & $n$ \\
\hline 6 & 0 & 6 & 0 & $n$ & $n$ & $n$ & in & $n$ & $n$ & $n$ & $n$ & $n$ & $n$ \\
\hline$n$ & $n$ & $n$ & $n$ & $n$ & $n$ & $n$ & $n$ & $n$ & $n$ & $n$ & $n$ & $n$ & $\nabla$ \\
\hline$n$ & $n$ & $n$ & $n$ & $n$ & $n$ & $\nabla$ & $\nabla$ & $\nabla$ & $\nabla$ & $\forall$ & $\nabla$ & $\nabla$ & $\nabla$ \\
\hline$n$ & $\nabla$ & $\nabla$ & $\nabla$ & $\forall$ & $\forall$ & $\nabla$ & $\nabla$ & $\forall$ & $\forall$ & $\nabla$ & $\nabla$ & $\forall$ & $\forall$ \\
\hline ๙่ & สু & $\bar{a}$ & ฉ & ఎे & $\infty$ & $\infty$ & $\infty$ & $\infty$ & ১ & $\infty$ & $\infty$ & $\infty$ & 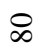 \\
\hline
\end{tabular}




\begin{tabular}{|c|c|c|c|c|c|c|c|c|c|c|c|c|c|}
\hline$r$ & 0 & $n$ & $n$ & $n$ & $n$ & $n$ & $n$ & $n$ & $n$ & $n$ & $\forall$ & $\nabla$ & $\nabla$ \\
\hline$r$ & 0 & 0 & $n$ & $n$ & $n$ & $n$ & $n$ & $n$ & in & $n$ & $n$ & $n$ & $n$ \\
\hline$\infty$ & $r$ & 6 & 6 & 0 & 6 & $n$ & $n$ & $n$ & in & $n$ & $n$ & in & in \\
\hline$\infty$ & $r$ & 6 & 6 & 0 & 6 & 6 & 6 & $n$ & $n$ & $n$ & $n$ & $n$ & in \\
\hline$\infty$ & $r$ & 6 & 6 & 0 & 6 & 6 & 6 & 6 & $n$ & $n$ & $n$ & $n$ & $n$ \\
\hline$\infty$ & $r$ & 0 & 6 & 0 & 6 & 6 & 6 & $n$ & $n$ & $n$ & $n$ & $n$ & $n$ \\
\hline$\infty$ & $r$ & 0 & 6 & 0 & 6 & $n$ & $n$ & $n$ & $n$ & $n$ & $n$ & $n$ & $n$ \\
\hline$r$ & $r$ & 6 & $n$ & $n$ & $n$ & $n$ & $n$ & $n$ & $n$ & $n$ & $n$ & $n$ & $n$ \\
\hline$r$ & 0 & $n$ & $n$ & $n$ & $n$ & $n$ & $n$ & $n$ & $n$ & $n$ & $n$ & $n$ & $\forall$ \\
\hline$r$ & 0 & $n$ & $n$ & $n$ & $n$ & in & $n$ & $n$ & $n$ & $\nabla$ & $\nabla$ & $\nabla$ & $\nabla$ \\
\hline$N$ & 0 & $n$ & $n$ & $\nabla$ & $\forall$ & $\nabla$ & $\forall$ & $\forall$ & $\forall$ & $\nabla$ & $\nabla$ & $\nabla$ & $\nabla$ \\
\hline 0 & $n$ & $\forall$ & $\nabla$ & $\nabla$ & $\forall$ & $\nabla$ & $\nabla$ & $\nabla$ & $\forall$ & $\nabla$ & $\nabla$ & $m$ & $m$ \\
\hline 6 & $n$ & $\nabla$ & $\nabla$ & $\nabla$ & $\nabla$ & $m$ & $m$ & $m$ & $m$ & $m$ & $m$ & $m$ & $m$ \\
\hline$n$ & $n$ & $m$ & $m$ & $m$ & $m$ & $m$ & $m$ & $m$ & $m$ & $m$ & $m$ & $N$ & $N$ \\
\hline$n$ & $\nabla$ & $m$ & $m$ & $m$ & $N$ & $N$ & $N$ & $N$ & N & $\sim$ & $\sim$ & $N$ & $\mathrm{~N}$ \\
\hline$n$ & $\forall$ & $N$ & $\sim$ & N & $\sim$ & $\sim$ & N & $N$ & - & - & - & - & - \\
\hline$\nabla$ & $m$ & $N$ & - & - & - & - & - & - & - & 0 & 0 & 0 & 0 \\
\hline$\forall$ & $m$ & - & - & 0 & 0 & 0 & 0 & 0 & 0 & 0 & 0 & 0 & 0 \\
\hline$m$ & $N$ & 0 & 0 & 0 & 0 & 0 & 0 & 0 & 0 & 0 & 0 & 0 & 0 \\
\hline $\mathfrak{n}$ & 으 & $\sqrt[n]{ }$ & సิ & 9 & $\infty$ & 工 & $\underline{0}$ & $n$ & 士 & 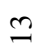 & $\simeq$ & 二 & 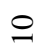 \\
\hline
\end{tabular}




\begin{tabular}{|c|c|c|c|c|c|c|c|c|}
\hline$\nabla$ & $\forall$ & $\nabla$ & $\nabla$ & $\forall$ & $\nabla$ & $m$ & $m$ & $m$ \\
\hline in & n & $\nabla$ & $\nabla$ & $\forall$ & $\nabla$ & $\nabla$ & $\nabla$ & $m$ \\
\hline n & $n$ & $n$ & in & $\forall$ & $\nabla$ & $\forall$ & $\forall$ & $m$ \\
\hline$n$ & $n$ & $n$ & $n$ & $n$ & $\nabla$ & $\nabla$ & $\nabla$ & $\forall$ \\
\hline$n$ & $n$ & $n$ & $n$ & $n$ & $n$ & $\nabla$ & $\forall$ & $\forall$ \\
\hline$n$ & $n$ & $n$ & in & $n$ & $\forall$ & $\nabla$ & $\forall$ & $\forall$ \\
\hline$n$ & n & in & $n$ & $\forall$ & $\forall$ & $\nabla$ & $\nabla$ & $m$ \\
\hline$n$ & $n$ & $n$ & $\nabla$ & $\forall$ & $\forall$ & $\nabla$ & $\forall$ & $m$ \\
\hline$\nabla$ & $\forall$ & $\nabla$ & $\nabla$ & $\forall$ & $\forall$ & $m$ & $m$ & $m$ \\
\hline$\nabla$ & $\forall$ & $\nabla$ & $\nabla$ & $\forall$ & $m$ & $m$ & $m$ & $N$ \\
\hline$\forall$ & $\checkmark$ & $\checkmark$ & $m$ & $m$ & $m$ & $m$ & N & N \\
\hline$m$ & $m$ & $m$ & $m$ & $m$ & $m$ & $N$ & $N$ & - \\
\hline$m$ & $n$ & $m$ & N & $N$ & $N$ & $N$ & - & 0 \\
\hline$N$ & $N$ & $N$ & N & $N$ & - & - & 0 & 0 \\
\hline$N$ & - & - & - & - & 0 & 0 & 0 & 0 \\
\hline- & - & 0 & 0 & 0 & 0 & 0 & 0 & 0 \\
\hline 0 & 0 & 0 & 0 & 0 & 0 & 0 & 0 & 0 \\
\hline 0 & 0 & 0 & 0 & 0 & 0 & 0 & 0 & 0 \\
\hline 0 & 0 & 0 & 0 & 0 & 0 & 0 & 0 & 0 \\
\hline$a$ & $\infty$ & r & 6 & $n$ & $\forall$ & $m$ & N & - \\
\hline
\end{tabular}

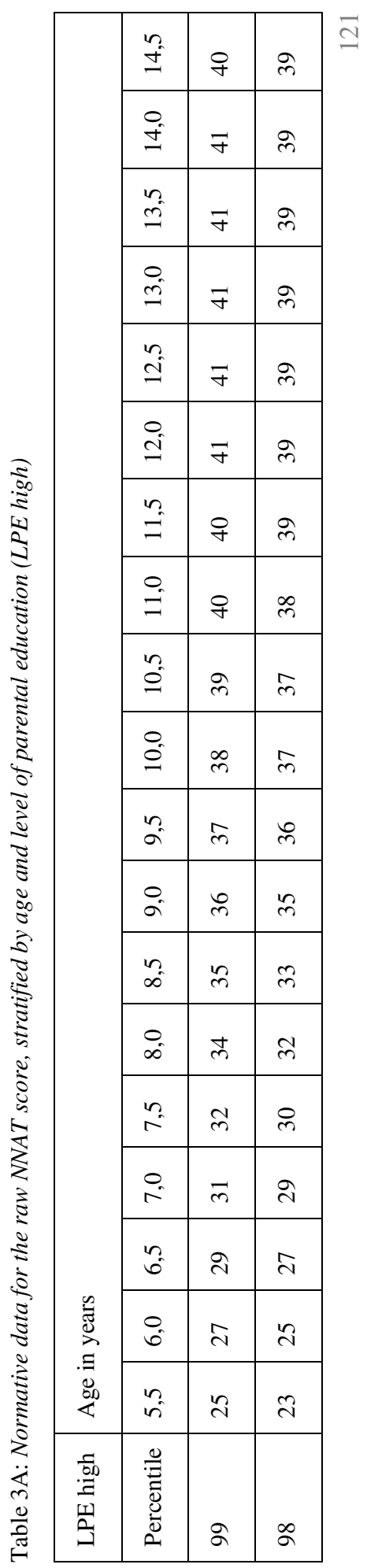




\begin{tabular}{|c|c|c|c|c|c|c|c|c|c|c|c|c|c|}
\hline$\stackrel{\infty}{\infty}$ & $\hat{m}$ & ల & $m$ & $\ddot{m}$ & मे & $\stackrel{\vec{m}}{ }$ & $\dot{m}$ & 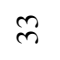 & $m$ & $m$ & $\tilde{\omega}$ & तै & $\approx$ \\
\hline$\stackrel{\infty}{\infty}$ & $\hat{m}$ & $\stackrel{r}{r}$ & i & $\ddot{n}$ & $m$ & dे & mे & $\dot{m}$ & $m$ & $m$ & $m$ & $\tilde{\infty}$ & $\approx$ \\
\hline$\stackrel{\infty}{m}$ & $\hat{n}$ & $\hat{n}$ & లৃ & $\ddot{m}$ & $n$ & মे & ষ্ & m & $m$ & $m$ & $m$ & 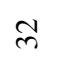 & $\approx$ \\
\hline$\underset{\infty}{\infty}$ & $\hat{m}$ & $\hat{m}$ & ల్ల & $\ddot{n}$ & $m$ & $\check{m}$ & $\dot{m}$ & $\dot{m}$ & $m$ & $m$ & $m$ & $m$ & $\approx$ \\
\hline$\stackrel{\infty}{\infty}$ & $\hat{m}$ & i & ల) & $\ddot{n}$ & $m$ & $\stackrel{\vec{m}}{ }$ & "े & $\tilde{m}$ & $\tilde{m}$ & $m$ & $m$ & ஸे & $\approx$ \\
\hline$\stackrel{\infty}{\infty}$ & $\hat{m}$ & r & లి & $\ddot{n}$ & $m$ & $\dot{m}$ & $\dot{m}$ & $m$ & $m$ & $m$ & $m$ & $\tilde{m}$ & $\approx$ \\
\hline$\hat{m}$ & $\hat{m}$ & i & $\stackrel{n}{m}$ & $\stackrel{n}{n}$ & $\dot{m}$ & $\dot{m}$ & $m$ & $m$ & $m$ & $\tilde{m}$ & $\tilde{\omega}$ & $\tilde{m}$ & लै \\
\hline$\hat{m}$ & $\stackrel{p}{n}$ & $\ddot{m}$ & $\ddot{n}$ & $\dot{m}$ & $\dot{m}$ & $m$ & $m$ & $m$ & $\approx$ & $\pi$ & $\tilde{\omega}$ & $\bar{m}$ & $\vec{m}$ \\
\hline i & $\ddot{m}$ & $m$ & 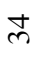 & $\vec{m}$ & $m$ & $m$ & సี & $\tilde{m}$ & तै & $\bar{m}$ & $\bar{m}$ & $\vec{m}$ & i \\
\hline$m$ & $\ddot{n}$ & $\dot{m}$ & $m$ & 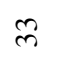 & $\tilde{m}$ & $\tilde{m}$ & $\tilde{m}$ & $\bar{m}$ & $\bar{m}$ & in & i̊ & in & in \\
\hline$\ddot{m}$ & ले & $m$ & ल & $\approx$ & $\bar{m}$ & $\bar{m}$ & $\bar{m}$ & $\stackrel{p}{ }$ & 尺 & ల & ते & Әे & ¿े \\
\hline$m$ & $m$ & లె & $\bar{m}$ & $\bar{m}$ & i & i & ते & ¿े & $\stackrel{\curvearrowright}{ }$ & $\stackrel{\infty}{\sim}$ & $\stackrel{\infty}{\sim}$ & $\stackrel{\infty}{\sim}$ & $\stackrel{\infty}{\sim}$ \\
\hline$\approx$ & $\bar{m}$ & $\bar{m}$ & i & in & ৯ิ & તે & $\stackrel{\infty}{\sim}$ & $\stackrel{\infty}{\sim}$ & $\stackrel{\infty}{\sim}$ & $\hat{\lambda}$ & $\hat{\sim}$ & $\bar{\lambda}$ & $\stackrel{\sim}{\sim}$ \\
\hline $\bar{m}$ & 尺) & ते & ते & $\stackrel{\infty}{\sim}$ & $\stackrel{\infty}{\sim}$ & $\hat{\lambda}$ & $\hat{\lambda}$ & $\stackrel{\sim}{\sim}$ & $\stackrel{\sim}{\sim}$ & $\stackrel{i}{ }$ & $\stackrel{i}{ }$ & $\check{\sim}$ & $\ddot{\imath}$ \\
\hline ते & $\stackrel{\infty}{\sim}$ & $\stackrel{\infty}{\sim}$ & $\hat{\lambda}$ & $\hat{\imath}$ & $\stackrel{\sim}{\sim}$ & $\stackrel{i}{ }$ & $\approx$ & $\ddot{\sim}$ & $\ddot{\sim}$ & d & ¿ & $\stackrel{\Xi}{\sim}$ & $\tilde{\lambda}$ \\
\hline$\stackrel{\infty}{\sim}$ & $\hat{\lambda}$ & $\underset{\sim}{*}$ & $\ddot{n}$ & $\sqrt[a]{a}$ & $\stackrel{J}{\Delta}$ & $\stackrel{d}{4}$ & $\stackrel{J}{d}$ & $\ddot{\lambda}$ & $\ddot{\lambda}$ & $\tilde{\lambda}$ & $\tilde{N}$ & 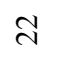 & ปี \\
\hline$\stackrel{\sim}{\sim}$ & $\ddot{\sim}$ & $\stackrel{J}{\sim}$ & $\stackrel{t}{d}$ & $\ddot{\lambda}$ & $\tilde{\imath}$ & $\tilde{N}$ & $\widetilde{N}$ & $\bar{\sim}$ & $\bar{\sim}$ & $\vec{\lambda}$ & $\bar{v}$ & ㄱ. & ㄱ. \\
\hline$\stackrel{\sim}{\sim}$ & $\tilde{\imath}$ & $\tilde{N}$ & $\tilde{N}$ & $\bar{\lambda}$ & $\vec{\lambda}$ & ते & ి & $\stackrel{\sim}{ }$ & $\stackrel{2}{ }$ & 2 & 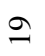 & $\stackrel{\infty}{\sim}$ & $\stackrel{\infty}{\sim}$ \\
\hline$\approx$ & $\vec{\lambda}$ & 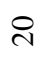 & নి & $\stackrel{2}{ }$ & $\stackrel{\imath}{ }$ & $\stackrel{\infty}{-}$ & $\stackrel{\infty}{\sim}$ & $\beth$ & $\beth$ & $=$ & $\stackrel{0}{0}$ & $\stackrel{0}{=}$ & $\stackrel{0}{-}$ \\
\hline$\hat{a}$ & ฉ̊ & $\stackrel{n}{\alpha}$ & d & $\sigma$ & สু & $\bar{\sigma}$ & \& & $\infty$ & $\infty$ & $\infty$ & $\infty$ & $\infty$ & \$ \\
\hline
\end{tabular}




\begin{tabular}{|c|c|c|c|c|c|c|c|c|c|c|c|c|c|}
\hline $\bar{n}$ & $\bar{m}$ & $\bar{m}$ & $\bar{m}$ & లి & $\ddot{\sim}$ & $\bar{\sim}$ & ㄱ. & 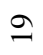 & 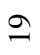 & 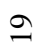 & $\stackrel{\infty}{-}$ & $\stackrel{\infty}{\sim}$ & $\stackrel{\infty}{\sim}$ \\
\hline ๙ & $\bar{m}$ & $\bar{m}$ & $\bar{m}$ & లి & $\approx$ & $\vec{\sim}$ & 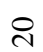 & 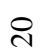 & ح & $\curvearrowright$ & $\curvearrowright$ & $\curvearrowright$ & $\cong$ \\
\hline$\stackrel{\sim}{\sim}$ & $\stackrel{\sim}{n}$ & $\vec{m}$ & $\vec{m}$ & ㅇ & 웅 & $\vec{\sim}$ & ㅇ & ㅇ & 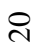 & 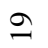 & 9 & 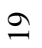 & $\infty$ \\
\hline N & N & $\bar{m}$ & $\bar{m}$ & లి & 우 & $\vec{\sim}$ & ๙ิ & ๙ิ & ণ & 9 & 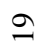 & 9 & 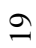 \\
\hline 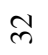 & $\bar{m}$ & $\bar{m}$ & $\bar{m}$ & లి & $\stackrel{n}{\sim}$ & $\vec{\sim}$ & ㄱ. & ㄱ. & 9 & 9 & $\stackrel{2}{ }$ & $\curvearrowright$ & $\infty$ \\
\hline$\approx$ & $\bar{m}$ & $\bar{m}$ & $\bar{m}$ & ஓ & $\approx$ & $\vec{\sim}$ & 이 & ๙ & 9 & 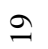 & $\stackrel{2}{ }$ & 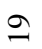 & $\stackrel{\infty}{\sim}$ \\
\hline $\bar{n}$ & $\bar{m}$ & $\bar{m}$ & $\bar{m}$ & ঐે & 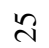 & $\vec{\sim}$ & 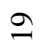 & 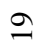 & $\stackrel{2}{ }$ & 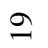 & 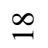 & $\infty$ & 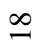 \\
\hline $\bar{m}$ & $\bar{m}$ & లి & లి & సे & $\stackrel{\Delta}{\sim}$ & ㅇ & $\cong$ & $\stackrel{2}{2}$ & $\infty$ & 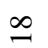 & 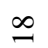 & 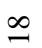 & 드 \\
\hline r & in & ㅇ & ते & $\underset{\sim}{\infty}$ & $\underset{d}{J}$ & 9 & $\stackrel{\infty}{\sim}$ & 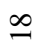 & $\stackrel{\infty}{-\infty}$ & $\stackrel{\infty}{\sim}$ & $I$ & I & I \\
\hline Әे & ঐे & Әे & ते & $\hat{\sim}$ & $\ddot{d}$ & 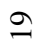 & 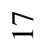 & 든 & 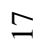 & I & $\underline{0}$ & $\stackrel{0}{-}$ & $\stackrel{0}{-}$ \\
\hline$\underset{\sim}{\sim}$ & $\underset{\sim}{\sim}$ & $\stackrel{\sim}{\sim}$ & $\stackrel{\sim}{\sim}$ & $\hat{\sim}$ & 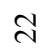 & $\stackrel{\infty}{\sim}$ & 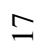 & $\underline{0}$ & 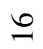 & $\underline{\sigma}$ & $\mathscr{0}$ & $\underline{n}$ & 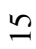 \\
\hline$\hat{\lambda}$ & $\hat{\lambda}$ & $\hat{\sim}$ & $\widehat{\lambda}$ & 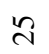 & $\vec{\sim}$ & 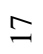 & $\cong$ & $\cong$ & $\cong$ & 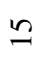 & \pm & $\Xi$ & $\Xi$ \\
\hline$\stackrel{\sim}{\sim}$ & $\stackrel{\sim}{\sim}$ & $\stackrel{\sim}{N}$ & $\sqrt[n]{ }$ & $\underset{\sim}{\Delta}$ & ิ & 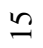 & \pm & \pm & \pm & $\underline{n}$ & $\underline{n}$ & $\stackrel{n}{=}$ & 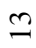 \\
\hline$\ddot{d}$ & $\stackrel{\forall}{d}$ & $\stackrel{d}{d}$ & $\stackrel{+}{\sim}$ & $\ddot{\sim}$ & $\stackrel{\infty}{\infty}$ & \pm & 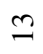 & $\stackrel{2}{2}$ & $\simeq$ & $\simeq$ & $\simeq$ & $\simeq$ & $=$ \\
\hline$\ddot{\sim}$ & 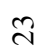 & $\ddot{\sim}$ & $\widetilde{N}$ & $\bar{\sim}$ & 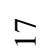 & $\stackrel{I}{ }$ & $=$ & $\Xi$ & $\Xi$ & $=$ & 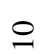 & 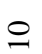 & \\
\hline ปี & $\vec{\sim}$ & $\vec{\sim}$ & $\vec{\sim}$ & ㄱ. & $\bumpeq$ & $=$ & 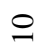 & $a$ & $a$ & $a$ & $a$ & $\infty$ & $\infty$ \\
\hline ㄱ. & 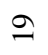 & $\curvearrowright$ & $\cong$ & $\stackrel{\infty}{\sim}$ & $\stackrel{m}{-}$ & $a$ & $\infty$ & $\infty$ & $r$ & $r$ & $r$ & $r$ & 0 \\
\hline$\stackrel{\infty}{\sim}$ & 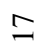 & 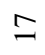 & 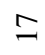 & $\stackrel{0}{ }$ & $=$ & $r$ & 0 & 6 & in & $n$ & $n$ & $n$ & $\nabla$ \\
\hline$\stackrel{\theta}{-}$ & $\cong$ & $\cong$ & $\stackrel{n}{n}$ & \pm & $a$ & $n$ & $\nabla$ & t & $m$ & $m$ & $m$ & $N$ & $N$ \\
\hline$\tilde{\infty}$ & ని & $\bar{\infty}$ & $\infty$ & $\stackrel{n}{\sim}$ & ஜn & $\sqrt[n]{\sim}$ & 요 & $\stackrel{2}{2}$ & $\infty$ & I & 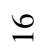 & $\cong$ & $\Xi$ \\
\hline
\end{tabular}




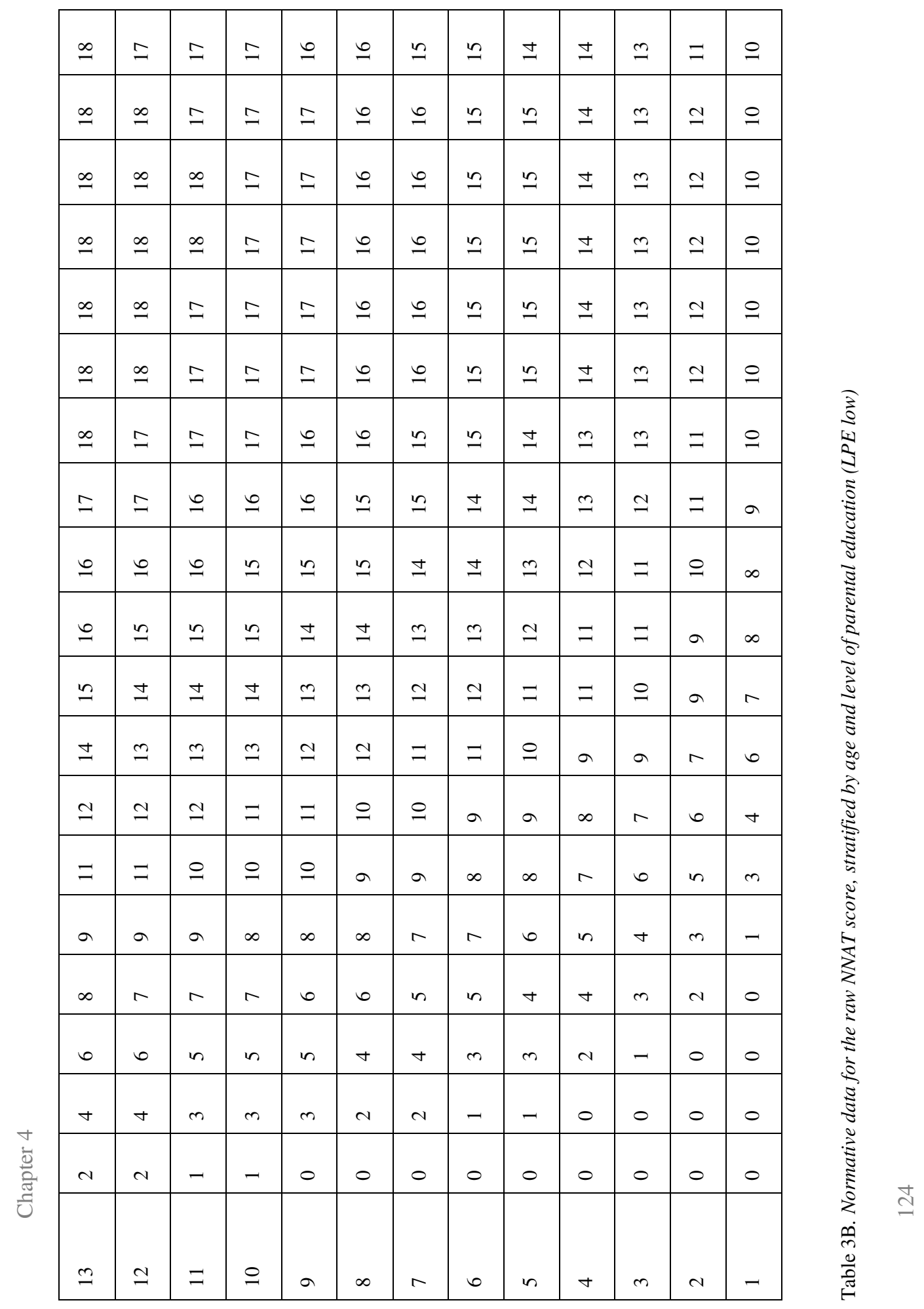




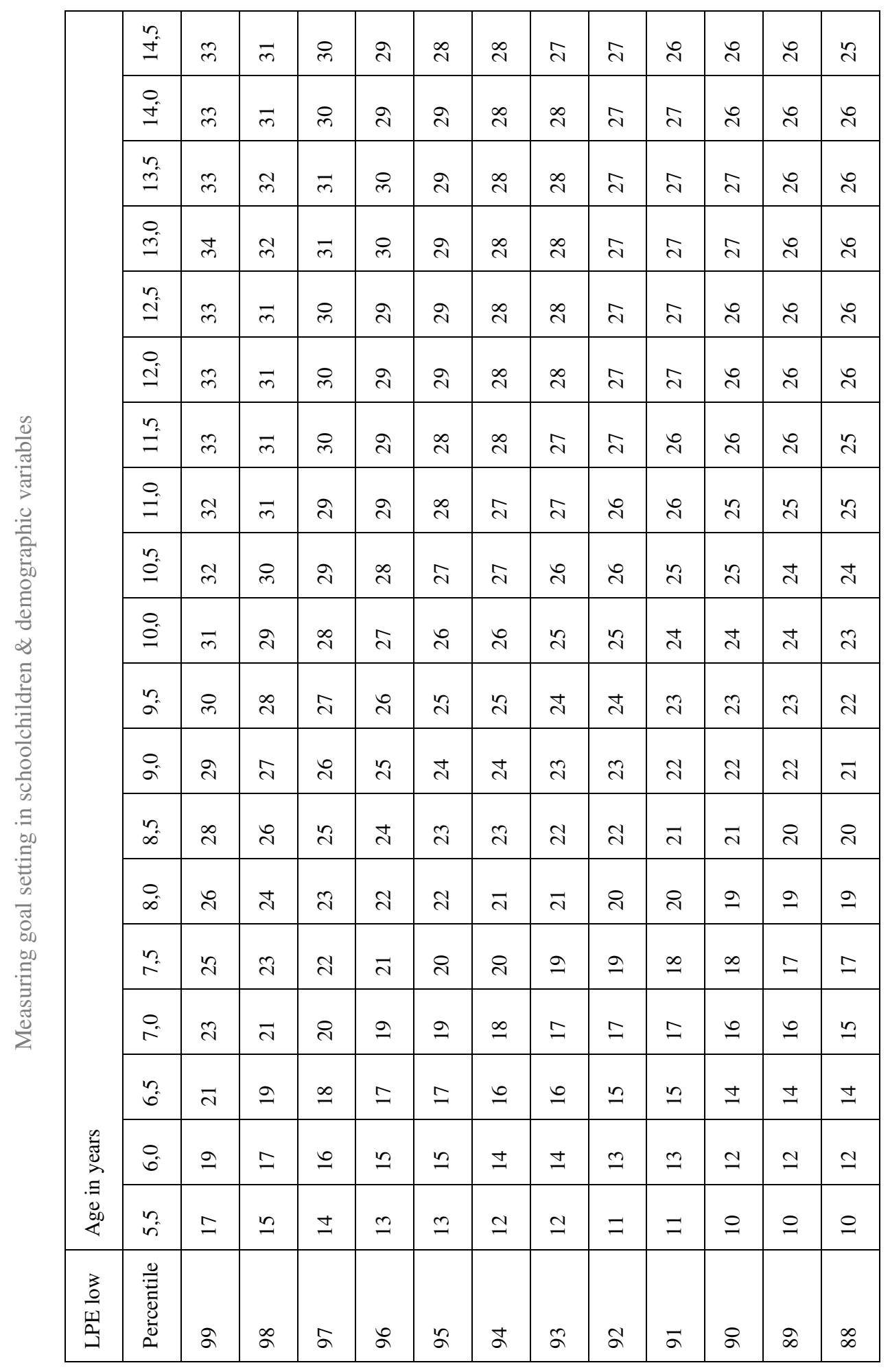




\begin{tabular}{|c|c|c|c|c|c|c|c|c|c|c|c|c|c|}
\hline$\dddot{\sim}$ & $\ddot{d}$ & $\stackrel{\sim}{\sim}$ & $\stackrel{\sim}{d}$ & $\stackrel{\sim}{\sim}$ & $\stackrel{\sim}{\sim}$ & $\tilde{\sim}$ & $\ddot{\lambda}$ & $\approx$ & $\stackrel{\infty}{\sim}$ & 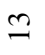 & $\simeq$ & $\simeq$ & $=$ \\
\hline$\ddot{a}$ & $\ddot{d}$ & $\ddot{\sim}$ & $\stackrel{\sim}{\sim}$ & $\stackrel{\sim}{\sim}$ & $\stackrel{\sim}{\sim}$ & $\stackrel{J}{\sim}$ & $\ddot{\sim}$ & $\approx$ & $\stackrel{\infty}{-}$ & 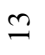 & $\simeq$ & $\simeq$ & $\simeq$ \\
\hline$\stackrel{\sim}{\sim}$ & $\ddot{d}$ & $\approx$ & $\ddot{\sim}$ & $\stackrel{\sim}{\sim}$ & $\stackrel{\sim}{d}$ & $\stackrel{\sim}{d}$ & $\stackrel{d}{d}$ & $\ddot{\theta}$ & $\stackrel{\infty}{\sim}$ & \pm & $\stackrel{m}{-}$ & $\simeq$ & $\simeq$ \\
\hline$\stackrel{\sim}{\sim}$ & $\ddot{d}$ & $\approx$ & $\ddot{\sim}$ & $\stackrel{\sim}{\sim}$ & $\stackrel{\sim}{d}$ & $\stackrel{\sim}{d}$ & $\stackrel{d}{d}$ & $\ddot{\theta}$ & $\stackrel{\infty}{\sim}$ & \pm & $\stackrel{m}{-}$ & $\simeq$ & $\simeq$ \\
\hline$\stackrel{\sim}{\sim}$ & $\approx$ & $\stackrel{\sim}{\sim}$ & 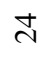 & $\stackrel{\unlhd}{\sim}$ & $\stackrel{\sim}{\sim}$ & 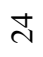 & $\ddot{\lambda}$ & $\widetilde{\sim}$ & $\infty$ & $\stackrel{m}{2}$ & 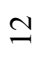 & $\simeq$ & $\simeq$ \\
\hline$\stackrel{2}{\sim}$ & $\stackrel{n}{2}$ & $\stackrel{2}{\sim}$ & $\stackrel{\sim}{\sim}$ & $\stackrel{\sim}{\sim}$ & $\stackrel{\mathbb{N}}{ }$ & 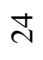 & $\ddot{\lambda}$ & $\widetilde{N}$ & $\cong$ & $\cong$ & 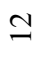 & $\simeq$ & $\simeq$ \\
\hline$\stackrel{\sim}{\sim}$ & $\stackrel{n}{a}$ & $\stackrel{d}{\Delta}$ & 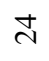 & $\underset{\sim}{\sharp}$ & $\underset{\sim}{\sharp}$ & $\ddot{\sim}$ & $\ddot{\lambda}$ & 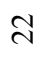 & $\beth$ & $\cong$ & $\simeq$ & $\simeq$ & $=$ \\
\hline$\stackrel{\sim}{\sim}$ & $\underset{\sim}{\sharp}$ & $\stackrel{\sim}{\sim}$ & 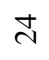 & $\ddot{\lambda}$ & $\widetilde{\lambda}$ & $\ddot{\sim}$ & $\ddot{\lambda}$ & $\vec{\sim}$ & $\beth$ & $\cong$ & $=$ & $=$ & $=$ \\
\hline$\stackrel{\sim}{\sim}$ & $\ddot{\lambda}$ & $\ddot{\lambda}$ & $\ddot{\sim}$ & $\ddot{\sim}$ & ป & ป & 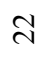 & $\bar{\sim}$ & 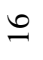 & $\simeq$ & $=$ & $=$ & 이 \\
\hline$\widetilde{\sim}$ & $\ddot{\lambda}$ & $\widetilde{U}$ & ป & 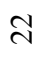 & $\widetilde{\sim}$ & $\vec{\sim}$ & $\vec{\sim}$ & ¿ & $\varrho$ & $=$ & $\stackrel{ }{0}$ & 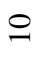 & $a$ \\
\hline$\stackrel{\sim}{N}$ & 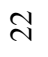 & $\vec{\sim}$ & $\vec{\sim}$ & $\bar{\sim}$ & $\vec{\sim}$ & ণิ & 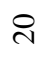 & 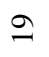 & 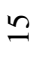 & $\stackrel{ }{-}$ & $a$ & $a$ & $a$ \\
\hline$\vec{\sim}$ & $\bar{\sim}$ & ণ & ๙ิ & ¿ & ণ & 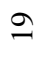 & 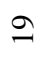 & $\infty$ & $\stackrel{m}{=}$ & $a$ & $\infty$ & $\infty$ & $r$ \\
\hline ণ & 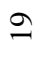 & $\stackrel{2}{ }$ & 9 & 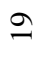 & $\stackrel{\infty}{\sim}$ & $\stackrel{\infty}{-}$ & $\infty$ & ㄱ & 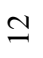 & $\infty$ & r & 6 & 0 \\
\hline 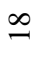 & $\stackrel{\infty}{-}$ & $\stackrel{\infty}{\sim}$ & 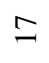 & $\beth$ & & 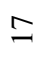 & $\underline{0}$ & 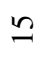 & $=$ & 0 & in & in & in \\
\hline$\beth$ & $\varrho$ & 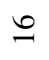 & 0 & $\underline{0}$ & $n$ & 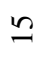 & 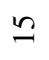 & $\Xi$ & $a$ & $n$ & $\nabla$ & $\nabla$ & $m$ \\
\hline 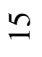 & $\cong$ & $\cong$ & $\Xi$ & $\Xi$ & $\Xi$ & $\stackrel{n}{=}$ & 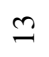 & 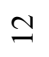 & $\infty$ & $m$ & $N$ & $N$ & $N$ \\
\hline$\stackrel{n}{=}$ & 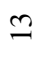 & 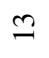 & $\simeq$ & $\simeq$ & $\simeq$ & $\simeq$ & $=$ & 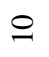 & 0 & - & 0 & 0 & 0 \\
\hline$=$ & $=$ & $=$ & & 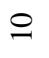 & 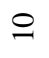 & $\stackrel{0}{=}$ & $a$ & $\infty$ & $\nabla$ & 0 & 0 & 0 & 0 \\
\hline$a$ & $a$ & $a$ & $\infty$ & $\infty$ & $\infty$ & $\infty$ & $r$ & 0 & $N$ & 0 & 0 & 0 & 0 \\
\hline$\hat{\infty}$ & $\triangleright$ & $\infty$ & + & $\tilde{\infty}$ & $\widetilde{\infty}$ & $\bar{\infty}$ & 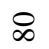 & 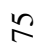 & in & $\sqrt{2}$ & ㅇ & $\cong$ & $\infty$ \\
\hline
\end{tabular}




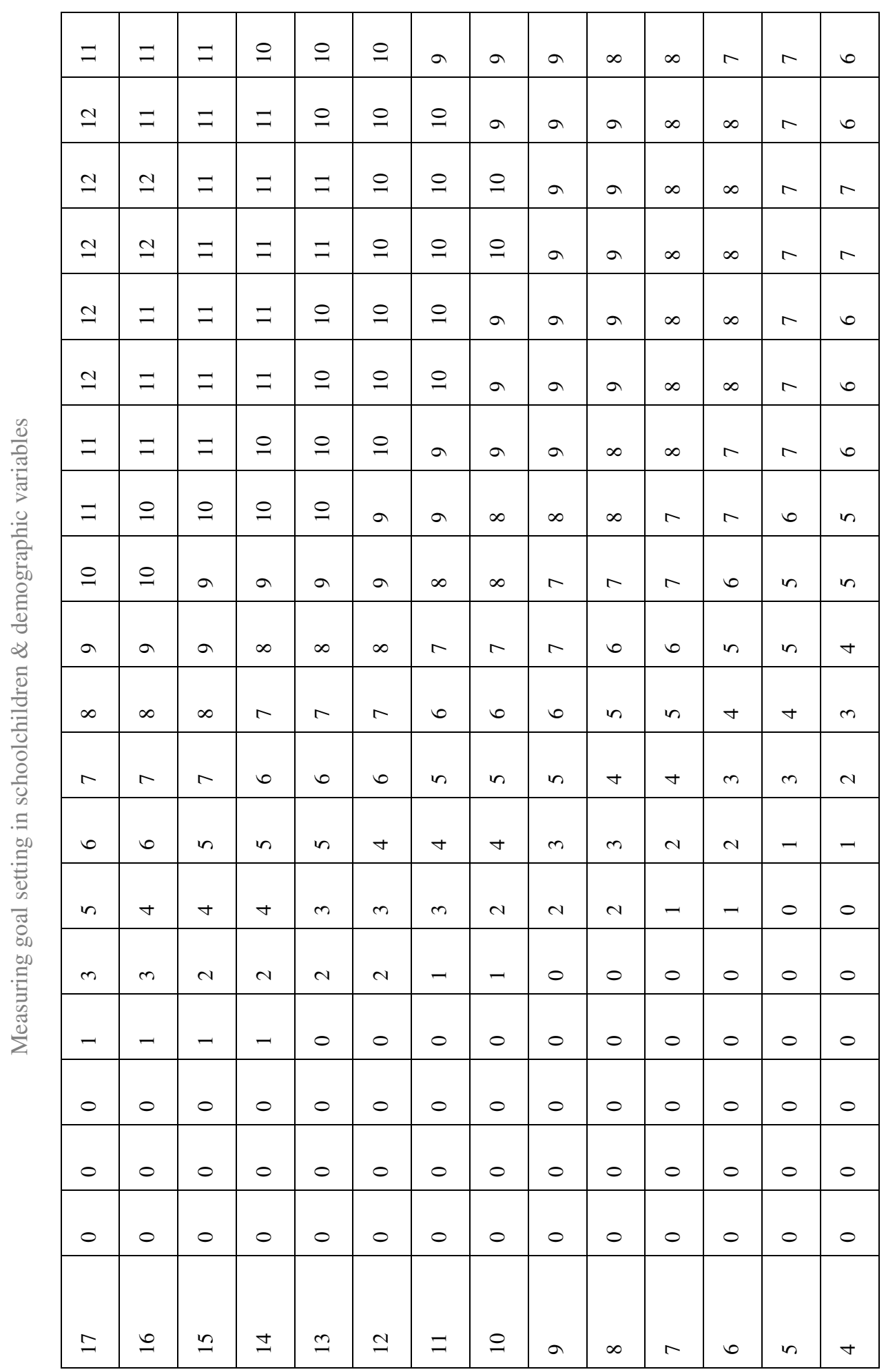




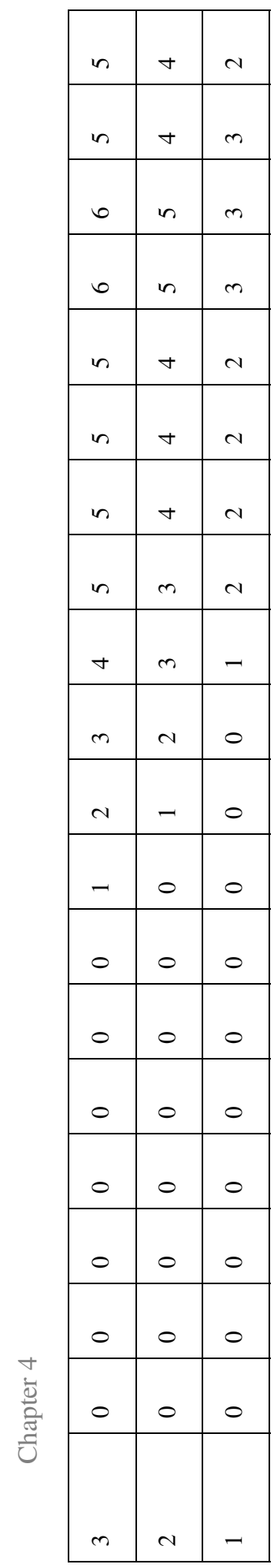




\section{CHAPTER 5}

Perceptions of alcohol and alcohol use in Western Ukraine: A study among community members and young adults

Resubmitted as: Toornstra, A., Massar, K., Hurks, P. P. M., Timmermans, M. M. M. S., Kok, G., \& Curfs, L. M. G. Perceptions of alcohol and alcohol use in Ukraine: A study among community members and young adults 


\title{
Chapter 5
}

\begin{abstract}
Background: Unhealthy alcohol use is a public health issue, prioritized by the World Health Organization (WHO) for prevention and reduction. Ukraine falls in the highest WHO category of 'years of life lost' due to alcohol use.

Objective: To investigate perceptions of alcohol consumption in a sample of youth and adults in Western Ukraine.

Methods: In-depth semi-structured interviews were conducted with 38 adult stakeholders (aged 21 - 63 years), and 81 adolescents and young adults (aged 12 -21 years) completed paper-and-pencil open-ended questions. A combined deductive-inductive thematic analysis of these qualitative data resulted in an initial coding scheme for both parts of the data. These initial codes were organized into patterns, which were further condensed to four themes.

Results: The four themes that emerged from the data were: The four themes that emerged from the data were: (1) The general historical, socio-economic-political situation and its relation to alcohol use, (2) Alcohol in the home and daily environment, (3) Alcohol use, related feelings, peers, family, and burden to health, and (4) Perceptions of the consequences of alcohol (mis)use.

Conclusions: Respondents indicated awareness that daily consumption, also in youth, as well as binge drinking and childhood sipping constitute a risk to health. These risks were described in the context of easy availability, low pricing, and peer pressure. The respondents mentioned awareness that alcohol dependence (AD) of parents affected families with the risk of disadvantageous child development. Interventions may include targeting current norms, stigmatizing beliefs and supporting subjects in developing coping skills.
\end{abstract}

Keywords: Alcohol dependence, alcohol related disease and harm, Ukraine, norms, health beliefs, stigma. 


\section{Introduction}

The burden of non-communicable disease allocated to alcohol misuse occupies the fourth place worldwide (World Health Organisation (WHO), 2018). Alcohol misuse has been associated with an increased risk of 200 chronic and acute diseases such as physical disease, mental disease, and consequences of prenatal alcohol exposure (Rehm et al., 2009). Alcohol has equally been implicated in (un)intentional harm, interpersonal violence, and alcohol dependence has been linked to stigma (Rehm et al., 2010; Schomerus et al., 2010). The most detrimental effects of alcohol on health are linked to the quantity and patterns of alcohol consumption and in particular to binge drinking. The highest rate of alcohol dependence (AD) associated with binge drinking are found in eastern European countries (Rehm, Shield, Gmel, Rehm, \& Frick, 2013). Ukraine falls in the highest WHO category of 'years of life lost' due to alcohol use, with an average alcohol consumption of 13.8 liters of pure alcohol per person per year (WHO, 2018). Legislation prohibits any alcohol use when driving and under 18 years old. There is, however no formal policy e.g., about the manufacturing or sales of alcohol. Consumption of unrecorded alcohol is suspected to be high (WHO, 2018).

Ukraine has a complex history before and currently a frozen conflict with Russia, which may affect economic pressures (Haukkala, 2015). It is considered to be a middleincome country; however, $3.3 \%$ of the population lives on $\$ 5$ per day or less and moderate poverty was $22 \%$ of the population in 2015 (World Bank, 2016; WHO, 2018). Alcohol misuse has been linked to poverty and is more common among marginalized populations (Hess, Frohlich, Calio, 2014; Jones, Bates, McCoy, \& Bellis, 2015). Research among young adults in Ukraine showed that especially those with high-risk occupations (e.g., coal mining) reported more frequent alcohol consumption, associated with coping with anxiety or stress, or as a result of social influence (Polshkova, Chaban, \& Walton, 2016). Equally, Novikova, Yaroslav, \& Olena (2019) raised concern about (the consequences of) social injustice and economic disadvantages for, among others, young and vulnerable people. Furthermore, increased alcohol consumption has also been linked to the quality of parenting (Burlaka, Grogan-Kaylor, Savchuk, \& Graham-Bermann, 2017), which affects children's well-being. Chambers et al. (2014) reported in their large sample of pregnant women in Ukraine that $97 \%$ were ever drinkers and $46 \%$ of those had consumed alcohol in the most recent month of pregnancy. In Ukraine the age of alcohol initiation is between 10-13 years old (Currie et al., 2004) which constitutes an increased risk for limitations in development, affecting learning and contributing to lower earning potential (Hellemans, Sliwowska, Verma, \& Weinberg, 2010; Luciana, Collins, Muetzel, \& Lim, 2013; Roozen et al., 2016; Steinhausen \& Spohr, 1998).

In summary, alcohol misuse and dependence may affect the health of the person and their family in a wide array of ways. The purpose of the current study was to make an inventory of perceptions of alcohol use, its relation to health, and whether these perceptions 


\section{Chapter 5}

differ between different stakeholders such as youths and adults in Ukraine. ${ }^{1}$ Knowledge of these perceptions may ultimately inform the development of interventions.

\section{Methods}

\section{Settings and respondents}

The research reported here was conducted in rural and urban areas; villages and cities stretching over an area of about 700 kilometers in Western Ukraine. This area has a population of Ukrainian, Hungarian, and some Roma, Ruthenean, or Russian inhabitants. We gathered our data in two ways: We conducted semi-structured in-depth interviews with 38 adult stakeholders (aged 21 - 63 years) from the communities and asked adolescents and young adults (aged $12-21$ years) to complete paper-and-pencil open-ended questions. People were invited to participate in the interviews, sampling a wide spectrum of stakeholders; people who misuse alcohol currently, family of person(s) who is (or was) misusing alcohol, health professionals, and people in varying community roles. Respondents reported a range of socio-economic and educational backgrounds from the highest completed level of grade 9 to medical doctor degrees. These roles and positions were determined based on the respondents' self-reports. For example, we included individuals who stated to have recovered from AD but we did not conduct checks whether this was indeed the case. Some community leaders held (in)formal positions, e.g., a mayor or a medical doctor involved in setting up private clinics.

Due to constraints imposed by pupils' curricular schedules at schools, we chose to administer semi-structured open-ended questions, which the pupils completed in their classrooms. The packet of questions was printed on paper, and included themes such as the perceptions of (mental) health, perceptions of alcohol use and the relationship between these factors. In constructing these questions, as well as the interview guide, we were guided by theories that attempt to explain behavior, and applied these theoretical constructs to our questions about perceptions of health, legislation, and subjective norms about alcohol (mis)use and alcohol dependence (see e.g., Bartholomew-Eldridge et al., 2016). Alcohol dependence $(\mathrm{AD})$ in this study is not a diagnostic term but is used to describe varying levels of alcohol misuse that interfere with daily activities in work- and social life and/or the need to consume alcohol. AD survivor is defined as people who overcame alcohol dependence as defined above. Alcohol misuse is not necessarily AD, and in the current paper we use the

${ }^{1}$ Stigma related to alcohol dependence in this sample is reported elsewhere. 
term to express the descriptions used by our respondents, for example to indicate binge drinking (Wechsler \& Kuo, 2000) or other forms of alcohol consumption that pose a risk to health.

The research ethics committee of the Faculty of Psychology and Neuroscience of Maastricht University, The Netherlands, approved this study. No compensation was provided to participants, participation was completely voluntary. Appropriate levels of consent were obtained from both samples before the data collections commenced.

Table 1. Sample composition (Note this table contains frequencies based on self-report

\begin{tabular}{|c|c|c|c|}
\hline & Rural & Urban & Age \\
\hline \multicolumn{4}{|l|}{ Interviews } \\
\hline People recovered from alcohol misuse & 3 & 7 & $18-60+$ \\
\hline Male / female & 6 & 4 & \\
\hline People at high risk of continued alcohol misuse & 1 & 6 & $30-55$ \\
\hline Male / female & 6 & 1 & \\
\hline Family of people misusing alcohol & 3 & 2 & $20-39$ \\
\hline Male / female & 0 & 5 & \\
\hline Community leaders & 3 & 3 & $30-65^{*}$ \\
\hline Male / female & 4 & 2 & \\
\hline Health Care professionals & 2 & 3 & $25-59$ \\
\hline Male / female & 4 & 1 & \\
\hline International volunteers \& professionals & 5 & 0 & $15-60+$ \\
\hline Male / female & 1 & 4 & \\
\hline \multicolumn{4}{|l|}{ Open-Ended Questions } \\
\hline 1 middle school & 1 & & $12-21$ \\
\hline Male / female & 9 & 8 & \\
\hline
\end{tabular}




\begin{tabular}{lcc}
\hline 1 high school & 1 & \\
Male / female & 10 & 16 \\
\hline 1 middle and high school & 1 \\
Male / female & 9 & 15 \\
\hline 1 college & 6 & 1 \\
Male / female & 6 \\
\hline
\end{tabular}

\section{Data collection and materials}

\section{In-depth interviews}

The first author conducted the interviews. Apart from one visit to one of the villages, there was no prior relationship with Ukraine. International NGOs and international workers introduced the researcher to translators. The researcher approached schools, colleges, and rehabilitation facilities with the help of translators, and approached individuals within these contexts. Some participants were invited from a selection of the population, such as individuals who reported having overcome alcohol dependence. Word of mouth recruitments comprised the main strategy, and often started with translators in the communities asking around for interested individuals (i.e., snowball and purposive sampling). The in-depth semi-structured interviews focused on perceptions of (harmful) alcohol use and its relationship to health in Ukraine, participant's local communities and their families. Moreover, the interviews focused on lived experience regarding alcohol (mis)use. Each interview lasted about an hour (some 1.5) and was conducted in a prearranged separate room with only the respondent, translator, and researcher present. All interviews were audio-recorded, and translations were once more verified during transcribing the recorded data.

The questions were translated, and participants received a written copy of the interview guide at the start of the interview to inform them about the content and provide them with the opportunity to propose changes. Next, the interviewer introduced the topic and asked the first question, which was simultaneously translated. The written questions served as a guide during the interview, but were utilized sparsely, because the respondents talked freely and spontaneously about their perceptions of and experiences with alcohol. Further explanations were asked or evoked using short prompts 'to tell a bit more about an issue'. Example questions are: 'What do you see in your environment in relation to alcohol (use)?', 'What do you consider to be 'healthy alcohol consumption' with respect to your age group?', and 'What are the disadvantages of alcohol consumption for your age group?'. 


\section{Open-ended questions}

The second sample consisted of middle school, high school, and college students attending the institutes that agreed to participate in the research. The pupils were invited to complete a set of open-ended questions during class. Students completed the questions independently, under supervision of a homeroom teacher, with the researcher being present. No further guidance was given unless the pupils asked questions. Completed forms were handed in face-down, not visible to teachers or the researcher.

The packet for pupils contained ten open-ended questions in total, covering the following topics: students' perceptions of their own health, healthy alcohol use (given their own age), the law and (inter)national (non-)adherence to legislated alcohol use, peers and alcohol consumption, and their thoughts on the prevention of alcohol misuse. These questions were constructed based on the Reasoned Action Approach, which proposes that attitudinal, normative, and control beliefs influence intentions, which in turn affect behaviors (Fishbein \& Ajzen, 2010). Example questions are: What does 'health' mean to you? and 'If you decide not to join a round of drinks, how would your peers react?' 2

\section{Data analysis}

The interviews and open-ended questions were analyzed using an inductivedeductive approach (Thomas, 2003). Transcribing ensured the researcher's familiarity with the data (Braun \& Clarke, 2006). Non-verbal information from the interviews was only included in the transcript if deemed different from the speaker's observed general demeanor, e.g., agitated tone of voice indicated by a higher pitch and pace of speaking. Semantic analysis was conducted next, which concerned the surface meanings of the data without implying meaning beyond the spoken/written words (Braun \& Clarke, 2006). All data were analyzed using NVivo 11.

Coding was conducted in two ways: First, some of the codes could be determined a-priori based on the interview protocol and the open-ended questions (e.g. 'health' or 'alcohol dependence'). Second, the complete data corpus (i.e. both the interview data and the answers to the open-ended questions) was analyzed line-by-line, and a multitude of basic segments formed initial codes that were grouped into larger categories (e.g., perceptions of alcohol use in rural areas, or perceptions of alcohol use and consequences for family members). Thematic analysis was applied to the complete data corpus to identify

${ }^{2}$ For the complete question list and brief overview of responses from the pupils, as well as matched responses from the interviews, see Appendix Table A. 


\section{Chapter 5}

and organize these initial coding into patterns (which were later condensed in the themes), without further consultation of research or theories, or the questions posed to the two samples of respondents (Braun \& Clarke, 2006). For each part of the data (transcripts versus open-ended questions), after initial coding was completed, all materials were again rigorously read, to confirm coding and especially to connect the two sets of data with each other based on the patterns that emerged (e.g., perceptions about health and alcohol use from the interviewees as well as from the adolescents and young adults). The analysis was thus conducted as a recursive process - a constant moving back and forth between the whole data set and the meaning of patterns (and later the (sub-) themes; Thomas, 2003; Braun \& Clarke, 2006). A second coder independently coded two interviews and ten student responses to verify the first coder's scheme. In case of disagreement (i.e., more than $20 \%$ on (a) theme(s)) a third independent coder was consulted.

The main aim of these analyses was to reflect reality, i.e. the lived experiences of participants. For example, potential mood disorders, describing behaviors in relation to alcohol misuse (without mentioning the word 'mood disorder') were reported by different groups of stakeholders concerning several family members in a household potentially affected by $\mathrm{AD}$ and subsequently reported as risks to health for families. Rather than interpreting these and drawing conclusions about the populations' mental health, we report these as stated by our respondents.

The four themes that emerged from the data were: (1) The general historical, socio-economic-political situation and its relation to alcohol use, (2) Alcohol in the home and daily environment, (3) Alcohol use, related feelings, peers, family, and burden to health, and (4) Perceptions of the consequences of alcohol (mis)use.

\section{Results}

Theme 1: General historical, socio-economic-political situation and relation to alcohol

Ukraine was described by the respondents as a geographical environment with a variety of natural resources, enough land and a good climate to grow food. Respondents in all professions reported they worked in their own vegetable garden to supplement a low income and/or provide in times of no income. One male community leader (35 years old) observed:

"Teachers did not get paid for a few years."

Respondents mentioned that many people did not have running water or toilets in their house and had to resort to charity-financed food services in winter. Multiple stakeholders described that people without observable income or bank accounts still manage to get by and they suggested this was a characteristic of a minority in Ukraine. As one female community leader (35 years old) described: 
"We know how to live through on thin ice."

Independence in 1991 was according to community leaders the end of a country that some had not anticipated, because they thought the USSR was the "mightiest" in the world. Following the end of the USSR, in Ukraine several collapses of financial systems ensued accompanied by significant losses of jobs, savings and pensions. Only a few of the respondents mentioned to have anticipated the independence and ensuing chaos and to have redirected their money. Local economies also collapsed resulting in an increased need to become self-sustainable while more people worked on their home farm due to a lack of jobs. Three health workers (males, 35-55 years old) reported this was not full-time work and that:

“... people would drink alcohol the rest of the day ... They cannot imagine that they can have a better life.”

Respondents remarked that outdated and still centrally organized politicaleconomic structures affect all citizens in their daily lives from the state of roads, and public transport, to access to medical supplies and the approach to work. According to community leaders some individuals longed for the old days when work and medical aid was certain for everyone and that people in their communities who still work according to communist norms struggle to understand that their work lacks standards in quality and quantity. On the other hand, community leaders, health professionals and international professionals reported about people who successfully made the challenging transition from communism to a free market economy through diligence, hard work and meticulous planning. Community leaders, international workers and an AD survivor described historical habits of payments for local work in (unrecorded) alcohol and reported that nowadays people stopped accepting payment in alcohol and demanded money, which generated sufficient income. However, others go abroad to gain income, which some link to alcohol use in females. An AD survivor (male, 33 years old):

"Here in Ukraine, lot of the men are working abroad, and usually the wife would stay at home with the children. It is a common problem that the wives get together and they start drinking alcohol. Some of them have depression, and if they would drink a little alcohol, she feels better, so she thinks she found herself a medication. ... She drinks an amount of alcohol and she is feeling better. This amount would get bigger and bigger. She doesn't realize that she is addicted to alcohol only her environment realizes it."

\section{Theme 2: Alcohol in the home and daily environment}

The next theme that arose from the data concerns 'a culture of alcohol consumption' as a structural part of daily life for many Ukrainians. Respondents mentioned that households grow their own grapes and make their own wine; the vines provide shade 


\section{Chapter 5}

for family meals, are accompanied by a glass of wine for all. Being able to make good wine is a rite of passage for males, subject to local competitions and part of family- and cultural celebrations. Wine is consumed throughout the day as if it were water; agricultural workers sip from a small plastic bottle. Sipping in pre-school children was reported three times; a female international volunteer (19 years old) doing community visits mentioned:

[Grandmother said] "... a few sips [of wine] does not matter", and "when she [5 years old girl] liked it, she got a whole glass."

In contrast, five adults reported their parents taught them abstinence when they were children up until 18 years old. Community leaders, family members and health workers reported that there used to be a 'healthy' culture of alcohol consumption, which consisted of enjoying a drink together, without misuse or binge drinking. They furthermore reported that, much of the current alcohol misuse could be linked to the end of strong state control after the collapse of communism. The majority of the young people however, stated that "healthy" alcohol consumption is not possible. AD survivors, community leaders and students reported that people in Ukraine 'do not have control' over their alcohol consumption once they start drinking or 'do not know their limits'.

From the interviews it became apparent that perceptions of alcohol use were intricately linked to specific local situations. Lower socio-economic households in rural areas collect drinking water from the local well. Historically it was perceived impolite to serve guests a drink containing water, as one might not be sure about its quality. Alcohol was preferred because it was perceived as 'clean'. Furthermore, beer was perceived as the drink to satisfy thirst and it is served at train stations on hot days. Beer is also seen as a drink for young people, females and "weak" people. It is sold in two-liter bottles in local supermarkets and described as cheap compared to beer in Europe. Four male and female AD survivors reported they already regularly consumed alcohol during their early adolescence (18-33 years old):

\section{"I drank beer from 12 years old and regularly from 14 years old."}

The strongest kind of alcoholic drink is vodka (or locally produced equivalents e.g., palinka), perceived as a drink for men and people who are "cool" and "strong". This labelling of 'being cool' was described in more than half of the answers to the questions completed by the youth, and it was in line with the interviews in which adults described alcohol consumption patterns in adolescence. These spirits are made from the leftovers of the fruit harvests or any cheap raw material such as sugar. A yearly harvest celebration of making palinka includes children as reported by an international professional couple (45 years old):

"... it is nice, all taste, including children ... 12 to 14 years old." 
Community leaders and older AD survivors state drinking 'vodka' is part of a culture that came from the east with (forced) migration or was the result of exposure to extremely strong alcohol concoctions during their one-year conscription. According to a female international volunteer (19 years old) high school boys said:

"We are Russian [...] ehm, Slavic culture, we can drink strong alcohol."

And a male AD survivor said (66 years old):

"And during that time in Russia you could buy those bottles of spirit in a simple shop. And as I got used to drinking wine at home and there in [...] I couldn't drink homemade wine. So, I didn't like the wine they made, ... . And the older soldiers taught me how to drink this spirit, this pure spirit."

When in company, multiple glasses are quickly consumed, and several AD survivors and community leaders reported having witnessed (or engaged in) excessive binge drinking. A female community leader (60+ years old) said that under communism much as men."

"... men drank a lot but were afraid to violate the rules" ... "now women drink as

AD survivors described stopping school at the earliest possible age (grade 9) and starting to work and that it was normal to consume alcohol before, during and after work. On the one hand Ukrainian customs proscribe that it is rude to refuse a drink, especially from an elder, but on the other hand, once one struggles to cope with the amount of alcohol - and becomes dependent on alcohol - one is shunned by others. A female family member (25 years old) said:

"There is no future if you are alcohol dependent."

A male AD survivor reported (44 years old):

“... and the people around me did not trust me anymore. It made me feel like I am alcoholic and I do not belong to the society."

Community leaders reported that they perceived AD in earlier behavioral stages (i.e., going binge drinking with peers after work). They explained that the frequency, type and amount of drinking alcohol might indicate problems. A male community leader (35 years old) reported:

"For our society it is normal to drink two to three times a week, but when you drink vodka like that, it is already alcoholic." 


\section{Chapter 5}

Interestingly two employers (an international couple and an AD survivor) who reported to have started a zero-tolerance policy on alcohol in their organizations stated few problems in implementing and demanding adherence to the rules. One report from a business in welding stated the need for safety and having dismissed one person over four years. In contrast family members, other AD survivors and international sources reported severe accidents at work.

Another source for daily and easy access to alcohol are local sellers. Family members, community leaders and health workers witnessed children being sent by a parent to local sellers to buy unrecorded alcohol. Local sellers also continued to sell these products, even when customers did not have any money, thus creating financial dependence. One female family member (20 years old) reported:

"They [local producers] even give wine to those who cannot pay in that time ... come back then next time you can pay ... in almost every village."

Additionally, respondents reported that youths can also purchase alcohol in shops and clubs. Multiple AD survivors, community leaders and students (in the open-ended questions) report regular alcohol consumption by youths between 12 - 14 years old. When children and teenagers go out any kind and amount of alcohol can be bought and drinks in clubs (including vodka) are only sold per bottle - increasing the risk of binge drinking. However, some individuals appeared aware of the harm of under-age drinking, since there were community reports of not selling alcohol to children. One female community leader (35 years old) stated:

"If I bought alcohol in the shop they would have called my parents straight away."

Another community managed to temporarily close the village shop that sold alcohol to children but could not implement lasting change. Some youths reported that in their view alcohol is more harmful to younger children as 'their bodies are more vulnerable'.

Theme 3: Alcohol use, related feelings, peer groups, families and burden to health

Students and AD survivors reported that alcohol consumption gives a feeling of being "happy", "free", and sharing "funny" moments together. A male community leader (35 years old) said:

"I think most of the 12 years old children feel themselves adult when they are drunk."

Some youths reported to drink alcohol to alleviate stress. Youth, however reported in 44 out of 81 cases that the group would convince them to drink alcohol, if needed forcefully by 
characterizing the abstainers as "not brave”, "stupid", "childish", or laugh at them. Youth reported group responses of stigmatizing and social exclusion:

"... they will look down on me", and "they will not talk to me", or "not be friends."

And a female student (15 years old) reported:

“They will ask you, are you a fool? Why don't you want to be grown up, to date boys?"

Some youths reported belonging to groups who do not consume or that the group accepts that they do not consume alcohol. About a third of youths linked it to their future goals of studying and reported (sometimes repeatedly) that alcohol use 'damages brain cells' and in their perception occurs from a young age. A male student described:

"By the age of 20 they will have been alcoholics with two young children."

Furthermore, apart from middle school students in a rural area, youth described in increasing detail (with age) that health for them encompasses physical and mental health and some added varying notions of well-being (e.g., social, spiritual; see appendix Table 1).

Young people, community leaders, family and AD survivors also linked absent parenting (i.e., 'not monitoring') to children's daily access to alcohol. Some reported absent parenting in connection to economic pressures (working long hours and/or abroad). A female AD survivor (18 years old) said:

"My parents did not have time to take care of me because they had to work all the time."

This same female AD survivor reported to start misusing alcohol from 9 years old, telling how her whole room would reek of alcohol the next morning. She said her parents would not investigate after her reply that it was "just beer". Negligent parenting was in about one third of the youths' responses linked to parental AD and its consequences, such as a lack of monitoring for their children's activities, and modeling alcohol (mis)use. Some youths reported that they thought parents need to give their children attention, which was confirmed by three reports from family and survivors. One male community leader who had grown up in an orphanage due to his father's AD described how parents do not really talk to their children, but are mainly telling them off:

"We do not know how to be families." 


\section{Chapter 5}

Families, AD survivors and community leaders reported episodes of intoxication and its consequences; deteriorating physical health (i.e., seizures), mental health problems of adults, significant physical violence toward family members, and children taking over parental duties. A female family member of a person experiencing AD (25 years old) stated:

"There is always a house in the street where there is screaming the whole night...

"Eight out of ten houses, you think it is normal."

An $\mathrm{AD}$ survivor (male, 60+ years old) reported:

"When I consumed alcohol, we were always arguing with my wife. When I came home drunk, she was very angry with me, of course."

Suicide and otherwise unnatural deaths were reported five times in this sample and in three cases concerned a first-degree family member. Family members described that the victims experienced $\mathrm{AD}$. One female family member described the moods as being 'worst when they wake up' after intoxication. One male AD survivor reported to have had suicidal thoughts before going in rehabilitation. A female family member of a person with AD (39 years old) stated:

"He [father] kept drinking all his life. When he hung himself, he was sober."

Families experiencing AD were reported to be seen as having a significantly lower socioeconomic status (SES), and to experience stigmatization and social exclusion. A female community leader (35 years old) reported:

"It is accepted in our society to look down on them..."

$\mathrm{AD}$ survivors and people currently at high risk of misusing alcohol reported after the initial sedative effects of alcohol consumption wore off they would feel "fear", "shame", "guilt", "feeling rejected", "powerless", "wanting to forget problems", and feel that there is no way out, including multiple attempts to stop consuming alcohol and undergoing 'inhumane' medical treatment. The latter was reported as 'being tied to a hospital bed' and receiving an injection that makes a person feel very sick on consuming alcohol, but its effects were described to wear off over time after which people started to (mis)use alcohol again. However, one male community leader (35 years old) said that men in their culture are not to talk about feelings:

"They only admit they have problems when they are drunk." 
Feelings of "loss" were related to losing jobs, or loss of families or their trust.

Family members and community leaders reported extreme poverty, which they thought was directly linked to the need to fulfill cravings for substances, e.g., they reported of individuals spending most of their salary in a binge drinking session with peers. Successful AD survivors describe after rehabilitation feelings of "happiness", "belonging", "peaceful", "strong", "wishing a normal life" and "not feeling judged". A female AD survivor (55 years old) said:

"I could start new things in my life which I hadn't done before."

The majority of AD survivors reported that they now understand they cannot consume any alcohol and expressed not to socialize with former peer groups.

Theme 4: Perceptions of the consequences of alcohol (mis)use

First, all stakeholders described impairments related to alcohol use during pregnancy in detail. A male community leader (35 years old) said:

"You can see it in their faces."

Multiple sources reported knowing about alcohol consumption in pregnancy. For example, the youth reported that the dangers of alcohol use in pregnancy were taught to them in high school. Asked about the causes of drinking during pregnancy, multiple sources implicated both the partner and the female. Two females (family members from unrelated families) who preferred to be interviewed together; 20 years old) reported:

"They go [to a bar] because their husband or boyfriend forced them to go", and "... "if a woman doesn't experience bad things when drinking alcohol in pregnancy then she will try and do it."

Health workers, family members and community leaders reported about children born in families experiencing $\mathrm{AD}$ and mentioned severe child neglect, orphanage- and state-family placements. Community leaders and health workers reported that they thought some appeared to have children in order to receive financial support (a lump sum paid at birth) and linked such motives to infant abandonment.

International volunteers and family members reported on people marrying young and having children in their early twenties, which they reported would result in unhappy marriages. These perceptions were also linked to views of limited academic success and economic options. In contrast to such perceptions, part of youth and several other younger adults in the interviews reported to prioritize higher education and to abstain from alcohol because of awareness that using alcohol might negatively affect their school performance. A female family member ( 25 years old) reported that she felt she was not more 


\section{Chapter 5}

academically talented than her peers, but succeeded in obtaining a university degree due to her choice of peer groups and her choice not to consume alcohol:

"All do it. Girls in my [middle/high school] class ... drink a bottle of wine between three of them in ten minutes ... then they go to a disco", and "it has the image of being cool."

Multiple sources reported a range of publicly or privately funded higher education options, which are also available for children from lower SES, but might be less so for children with behavioral and/or learning difficulties. A female community leader (30 years old) said:

"If they are not recommended by their pastor they are not allowed to study here."

About half of the youth reported that in their perception alcohol misuse consequences are not only in a distant future but affect people from early on in life: They described in varying detail behavioral changes, aggressive behavior, and mental- and physical health problems in general and specifically related to young people, such as starting to skip school and having learning problems. For example, according to youth these children from parents with AD:

"They hold the class back", and they "start to play truant", and "lying".

Teachers reported that some students exhibited more severe and persistent learning and behavioral problems. The summary of the results in the appendix reflects the differences between youths (open-ended questions) and adults (interviews).

\section{Discussion}

The purpose of the current research was to investigate which factors contribute to alcohol use in Ukraine, which role alcohol plays in Ukrainian society and how the consequences of alcohol (mis)use are perceived by a variety of stakeholders, including youths aged 12-21 years old. Several themes emerged from the semi-structured interviews and open-ended questions.

First, respondents perceived that general factors such as political-economic challenges linked to Ukraine's complex history and independence in 1991 were perceived as causes for alcohol misuse (Onwijn, 2005, p.40-58). Former Soviet countries, their historical-, geographic-, political challenges and interference with ethnic populations have been linked to structural problems in society associated with e.g., alcohol misuse (Herrick, 2012; Hess et al., 2014). The transition of 1991, as reported in this study resulted for some individuals in a struggle to change one's outlook on life. However, in contrast to such reports, half of the youth in this sample reported a long-term outlook on their future and 
making choices that support their plans and goals, including their health behaviors (e.g., not drinking alcohol).

Next, this multi-ethnic sample in Ukraine reported shifting norms concerning alcohol use. Perceptions that it used to be celebratory or people would consider strict public laws appeared changed. Descriptive norms in this sample, that is the unwritten rules in society that most follow (Cialdini, Reno, \& Kallgren, 1990), historically reflected a normality of daily and continuous alcohol consumption, but binge drinking was also reported. However, a Mediterranean culture of wine drinking with meals has been associated with the lowest volumes of alcohol consumption of 0.7 liters per person per year in Europe without acceptance of binge drinking (Popova, Rehm, Patra, \& Zatonski, 2007; WHO, 2018). This opposes quantities and patterns of alcohol intake reported in this sample. The Ministry of Health of Ukraine did not prescribe a minimum age for the consumption of beer until 2010 (Polshkova et al., 2016), and our respondents reported a custom of consuming beer to quench one's thirst and of children consuming wine at meal times. One local rehabilitation program instills the routine of consuming tea as a daily drink to quench thirst, including the knowledge about reduced risk to health from boiled water. Some of the norms and drinking habits can be linked to a culture of hospitality, celebrations and being a good Ukrainian citizen. Most respondents expressed limited coping strategies when confronted with such alcohol-related norms in their communities, especially in binge drinking situations or when elders insisted children should participate in celebratory alcohol consumption. These results indicate that alcohol (mis)use prevention need to include strengthening of self-efficacy to resist the pressure from elders and peers to drink alcohol.

Furthermore, a culture and a norm of early childhood access and -acceptance of alcohol consumption, including continuous and easy access (at low prices), emerged in this sample. This is in line with reports stating that in Ukraine $9.3 \%$ of boys and $3.1 \%$ of girls of 11 years old drink alcohol weekly and first episode of drunkenness was reported at 13.9 for boys and 14.6 years old for girls (Currie et al., 2004). However, given the anecdotal reports of our respondents about children sipping and drinking alcohol at younger ages, the actual age of onset for alcohol consumption might be lower. Research has shown that the age of onset of regular alcohol use is predictive of future problem drinking, other substance abuse, and developmental challenges (Hingson, Heeren, \& Winter, 2006; Hession, 2012). Also that the mental schemata that children form in the first few years of life such as parents' favorable attitudes about alcohol use and (early) childhood consumption predict childhood alcohol consumption onset (Donovan \& Molina, 2008; Zucker, Donovan, Masten, Mattson, \& Moss, 2008).

Moreover, the ability to deal with heavy irregular (binge) drinking was reported in this sample as a sign of 'strength'. These consumption patterns of consuming vodka or other spirits are associated with northern Europe and/or Slavic cultures and found to be most detrimental to health (Popova et al., 2007). In our sample, the AD survivors reported lengthy denial and a general underestimation of being at risk of becoming addicted as well as denying the supposed dangers of drinking large quantities of alcohol. Importantly, these patterns were associated with men, while Ukrainian men are not supposed to talk about 


\section{Chapter 5}

their feelings. Thus, the negative emotive states and consumption of alcohol 'to forget' indicate a limited ability to cope with stressors among men. The manner in which people deal with stress and/or anxiety has been linked to susceptibility of AD (Sinha, 2009).

Alcohol consumption was linked to lower satisfaction with the quality of the relationship between males and females and the risk of alcohol use in pregnancy (Bakhireva et al., 2011). Burlaka et al. (2017) found that $81 \%$ and $58 \%$ of Ukrainian women with school age children reported psychological or physical violence respectively from an intimate partner. This was associated among other factors with maternal lower level of education and unemployment in line with this sample's reports.

Likewise, disadvantageous parenting styles (i.e., inconsistent parenting), as described in this sample have been linked to alcohol use in adolescents (Barnow, Schuckit, Lucht, John, \& Freyberger, 2002). In this study alcohol use by youths was reported to reduce stress, which appear in line with literature on psychological distress (i.e., anxiety, depression) among 13-15 years old adolescents in developing countries (Balogun, Koyanagi, Stickley, Gilmour, \& Shibuya, 2014). Reports from this sample showed potentially multiple sources of stress for youths, i.e., parental AD, which may be associated with other (undiagnosed) mental problems (Hasin, Stinson, Ogburn, \& Grant, 2007). Furthermore, stigma attached to a family AD background as exhibited in general in this sample and by teachers and peers specifically, has been identified as a major source of stress for the person concerned (Bos, Pryor, Reeder, \& Stutterheim, 2013). Other adolescent challenges reported in our sample were peer pressure on non-consuming youths, which might be a response to possible limitations (i.e., exclusion) of future options such as access to a good high school or college due to limitations in academic results and/or behavioral problems. Alcohol consumption may serve ascertaining one's status in a group. Indeed, a limited education may be linked to the lower SES of an AD family background or 'working the land', which was mentioned by some respondents. A significant body of research shows the detrimental effects of adolescent binge drinking on brain functions, but cause and effect relations remain very difficult to establish (Luciana et al., 2013). A poorer neurocognitive performance (e.g., memory, attention, inhibition) was found in adolescents who meet criteria of exposure (Jacobus \& Tapert, 2013; Ware et al., 2015). Youths' reported learning differences in class coupled with reported perceptions of peers that struggling learners limit class progress, warrant the question whether children with learning difficulties need (more) adapted school programs?

Thus, to sum up, the broader patterns from these data point toward a combination of norms and common behaviors - acceptance of alcohol consumption, not talking about emotions, inter-relational violence - and limitations in adaptive coping skills to deal with feelings of anxiety, stress, and depression, and to resist peer pressure and to inhibit certain impulses. Together these may result in significant alcohol related health problems. Some children in the current sample appear at high accumulative risk for AD through these environmental- but also due to several biological factors. Some children face a developmental trajectory which includes prenatal alcohol exposure and its resulting effects on (mental) development and continue to early tasting of alcohol, and being subject to 
stigma if one comes from an 'AD family'. Such factors may put youth at risk for having to accept low-paying, high-risk jobs (Polshkova et al., 2016). Motives for alcohol consumption were reducing stress and anxiety, following friend's examples and to increase self-esteem.

Interestingly, in our sample successful people and families - that is those who have attained higher educational levels and/or successfully recovered from AD - report clear limitations in alcohol use or abstinence, building and maintaining relationships that sustain and enhance (Fiorillo \& Sabatini, 2011) and steadily working toward their goals. Importantly, half of youths reported varying notions of mental health as part of their concept of self and health. AD survivors for example, reported learnt coping skills and emotional well-being, which some described as a feeling of 'strength'. Successful people reported making protective choices for themselves and their environment, i.e., children are not allowed to sip alcohol or AD survivors choosing peers who understand their problems. Importantly, half of the youths reported knowledge and awareness of having to limit alcohol intake to achieve desired outcomes in life. Several respondents whose background showed multiple of the discussed risk factors report these protective (for some novel) choices, which might accumulate to reduce risk, resulting in advantageous life outcomes.

\section{Limitations}

This was a cross-sectional study in rural and urban (south) Western Ukraine, which prohibits causal inferences. The diversity of the sample resulted in a detailed description of alcohol consumption, perceptions of alcohol (mis)use and relations to health. However, the depth and breadth of alcohol misuse needs to be quantified in order to be able to generalize these findings to the larger Ukrainian population and to start addressing alcohol related health problems. There may be selection bias as word of mouth recruitment in communities started with translators (thus naturally their contacts) and the snowballing pattern was not analyzed (Biernacki \& Waldorf, 1981). However, the sample ranged from middle school to medical doctor degrees as highest level of education and contained a variety of stakeholders.

Next, other poverty related factors affecting development and quality of life, or which may interact with the variables we investigated cannot be excluded, e.g., effects of nutritional deficiencies on cognition, or daily stress of socio-economic uncertainty. Future research should include these.

\section{Conclusion}

Alcohol misuse in historically complex geographical locations has been associated with structural problems such as access to clean drinking water, work, continued education 


\section{Chapter 5}

for vulnerable groups, access to sufficient and appropriate rehabilitation programs, policy on alcohol pricing and availability, classifying of alcohol (beer), and unrecorded alcohol. Targeting unregistered artisan home-production (e.g., wine) poses the challenge of its central role in local cultural traditions and might benefit from a reward system for registration (Lachenmeier, Taylor, \& Rehm, 2011).

Some of the main themes in this research confirm that people's underlying beliefs determine their (health) behavior, which accordingly need to be targeted in health promotion interventions (Bartholomew-Eldridge et al., 2016). Knowledge in turn has been associated with these beliefs. Despite reported observational knowledge of devastating long-term outcomes of $\mathrm{AD}$ and/or alcohol misuse, in the current sample a lack of awareness of the effects of alcohol on one's body, a fetus, or young children's brains, the risk and consequences of binge drinking, and the susceptibility and risk of developing AD became evident. Importantly, the notion of control over one's health in a preventative manner appears to be comprehended by a small segment of this sample including part of youths. Similarly, one can only take care of one's mental health if there is an understanding what it is and if one feels self-efficacious to influence it (Bartholomew-Eldridge et al., 2016). The use of alcohol as a coping mechanism may indicate a lack of self-efficacy. A willingness and readiness to start discussing emotions may be further targeted with motivational interviewing techniques (Murphy, Dennhardt, Skidmore, Martens, \& McDevitt-Murphy, 2010). However, this needs to be accompanied by potential solutions which might be the individual's awareness that coping strategies can be learnt (Bartholomew-Eldridge et al., 2016) and creation of platforms for learning these skills. Equally, both individual and structural interventions are needed for male mental health given the extent of suicides reported in this sample alone, the perceived link to $\mathrm{AD}$, reported interpartner violence and proposed effects on the mental- and physical well-being of children. Mental health services for several at-risk groups (such as female victims of intimae partner violence) in this sample are equally important. Similarly, targetting adolesecent's coping strategies and selfesteem may benefit this target group. Indeed, coping skills and enhanced self-esteem were proposed by Polshkova et al. (2016) as targets for intervention to reduce risky behaviors and alcohol consumption to ease anxiety and stress in Ukrainian youths. Targeting adolescents could work preventively, i.e., the potential to link new coping strategies to advantageous parenting styles.

However, much of the above may have little effect as society's stigmatizing beliefs have been shown to form a major source of psychological distress, a barrier to seek help and causes of self-stigma ('to become like father'; Bos et al., 2013). This would require changing public perception, while simultaneously strengthening self-efficacy and self-esteem of the subjects of stigma (Schomerus et al., 2010). Perceptions of stigma are reported in an accompanying article. 


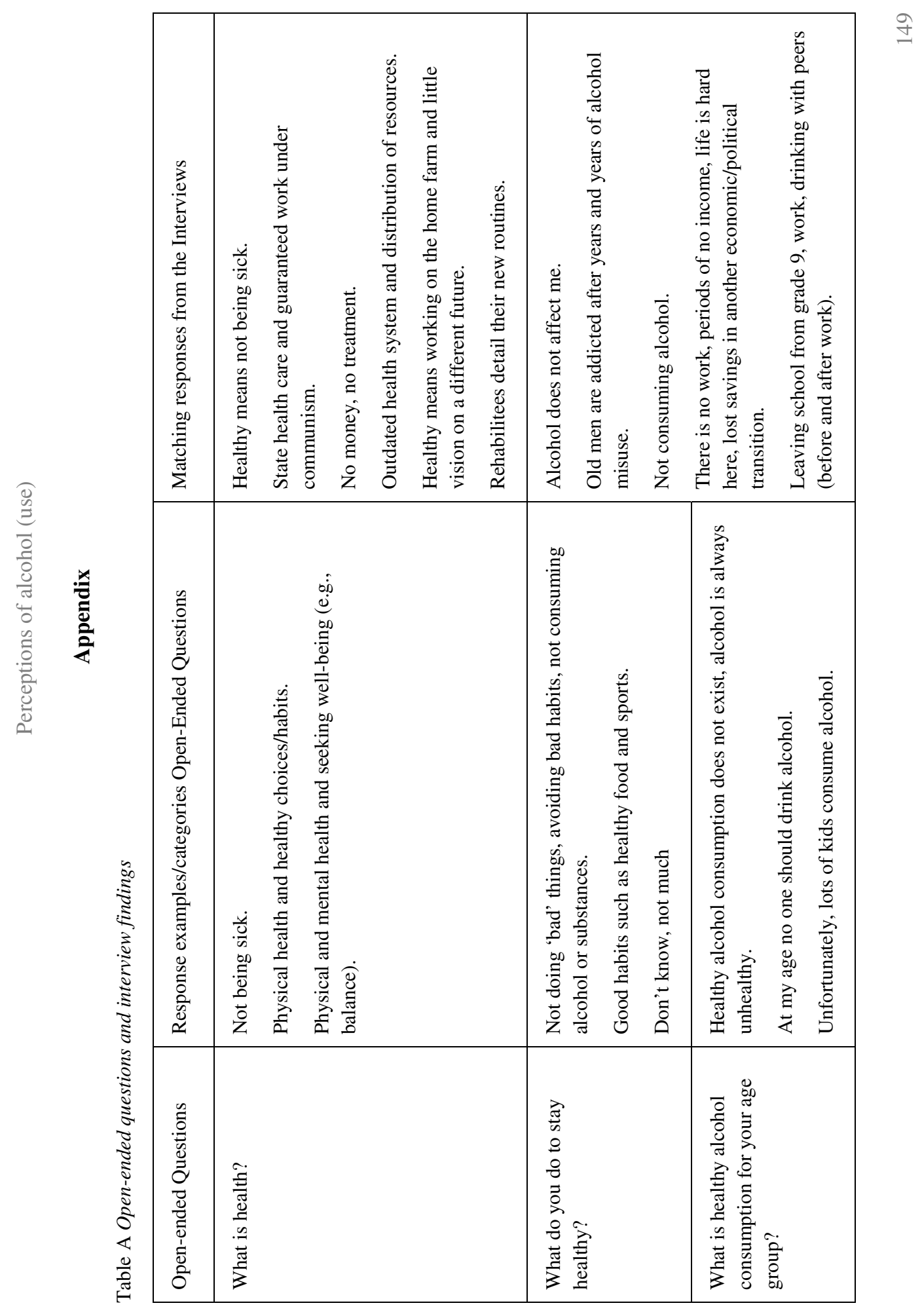




\begin{tabular}{|c|c|c|c|c|c|c|c|c|c|}
\hline 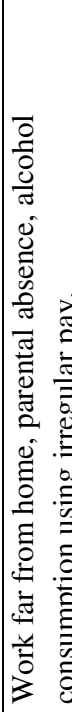 & 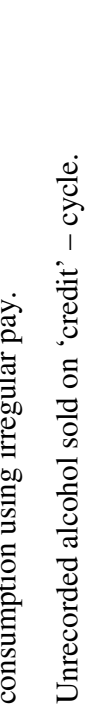 & 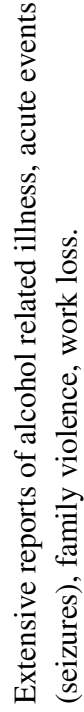 & 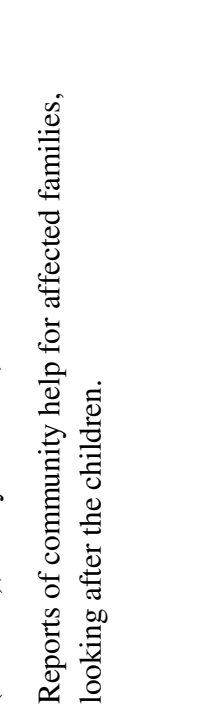 & 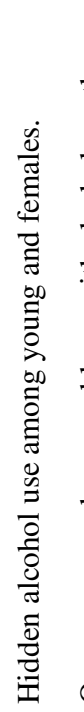 & 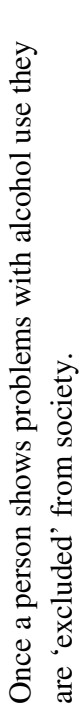 & 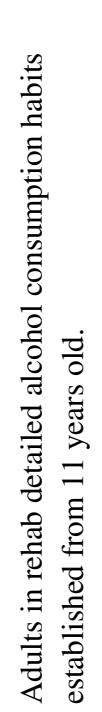 & 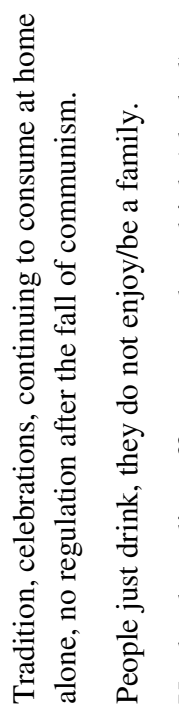 & 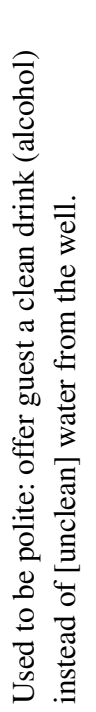 & 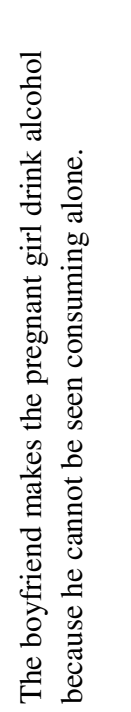 \\
\hline \multirow[t]{2}{*}{ 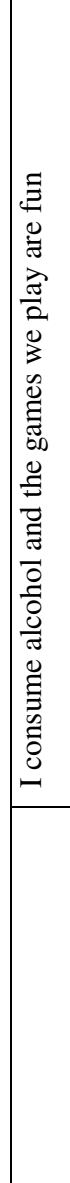 } & \multirow[t]{2}{*}{ 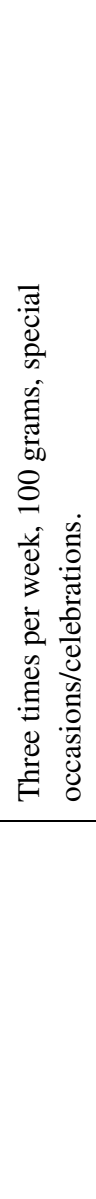 } & \multicolumn{2}{|c|}{ 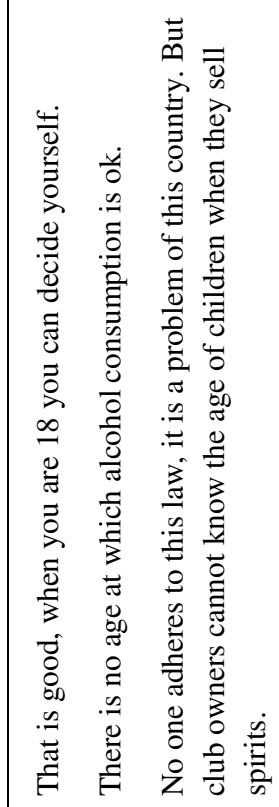 } & \multicolumn{3}{|c|}{ 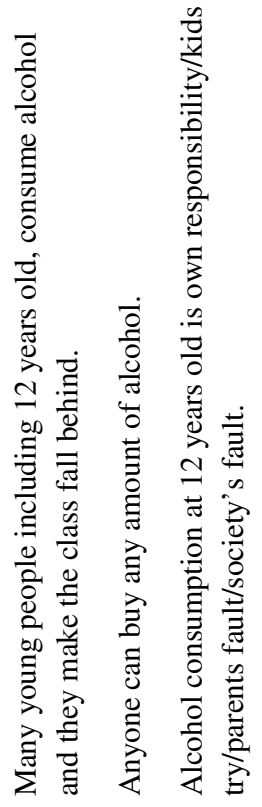 } & \multicolumn{2}{|l|}{ 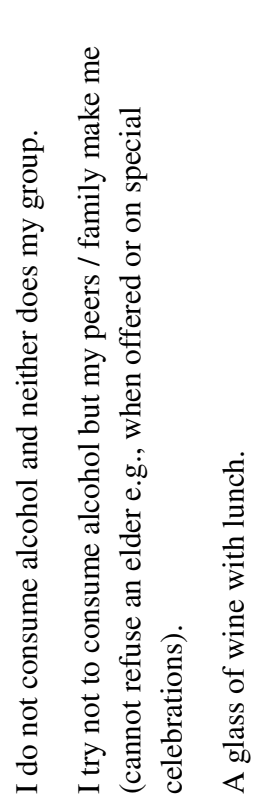 } & 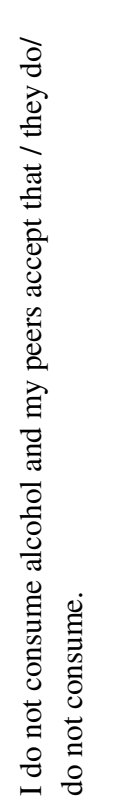 \\
\hline & & 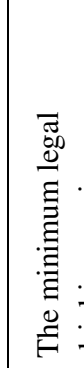 & 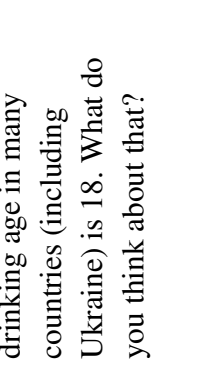 & 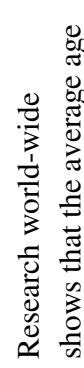 & 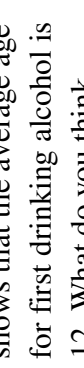 & & 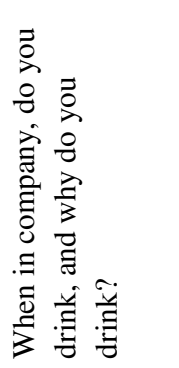 & & 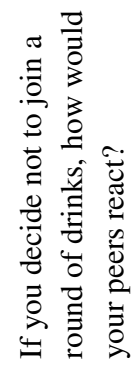 \\
\hline
\end{tabular}




\begin{tabular}{|c|c|c|c|c|c|c|c|c|c|c|c|c|c|c|}
\hline 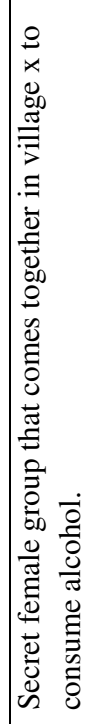 & 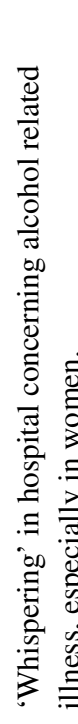 & 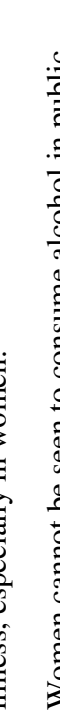 & 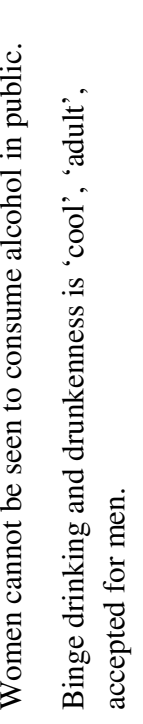 & 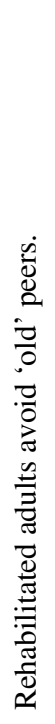 & 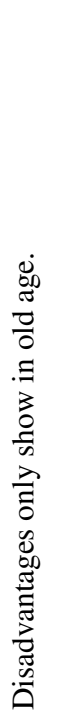 & 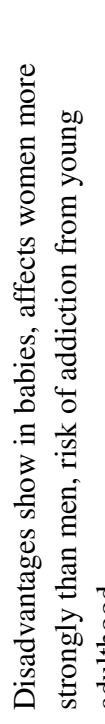 & & 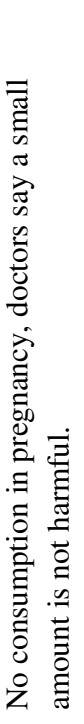 & & 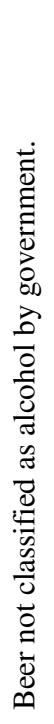 & 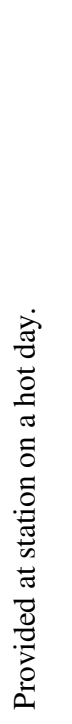 & 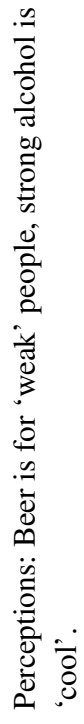 & 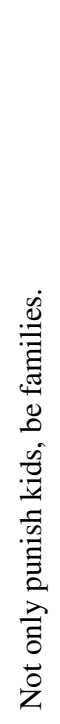 & 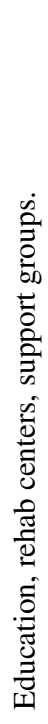 \\
\hline \multirow[t]{2}{*}{ 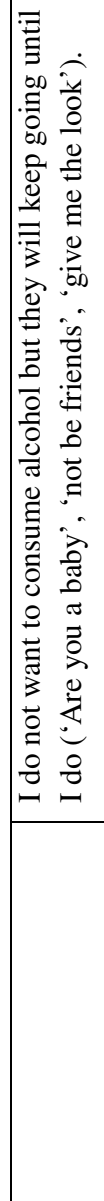 } & 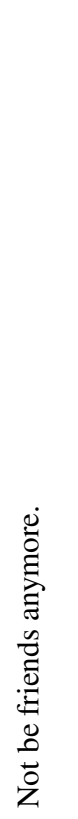 & 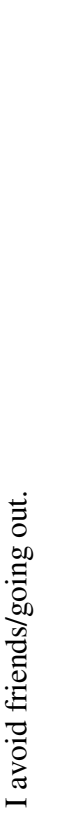 & 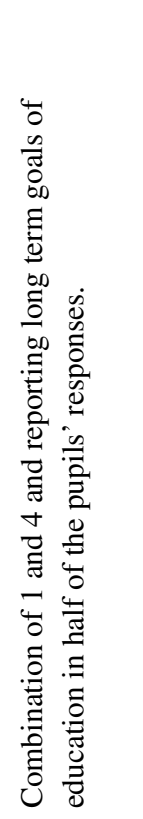 & & 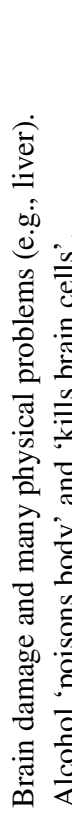 & 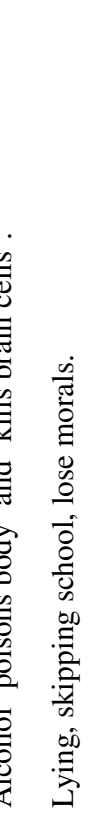 & 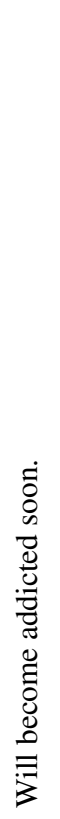 & 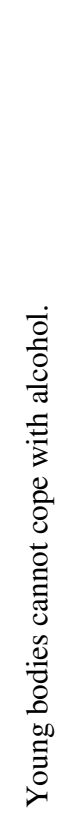 & $\begin{array}{l}0 \\
0 \\
0 \\
0 \\
0 \\
0 \\
0 \\
\vdots \\
0 \\
0 \\
0 \\
0 \\
0 \\
0 \\
0 \\
0\end{array}$ & 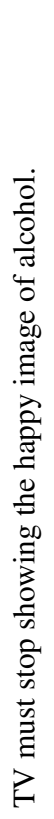 & 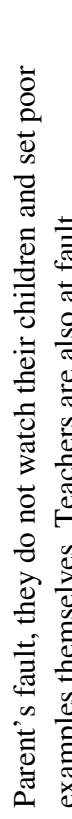 & 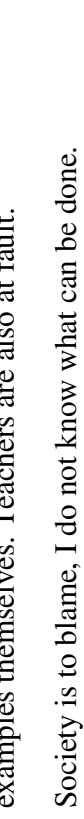 & 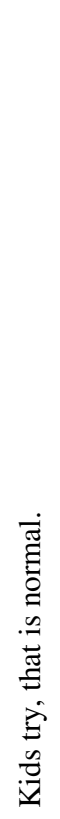 & 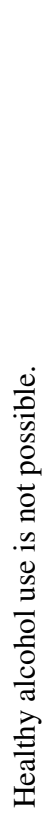 \\
\hline & & & & & 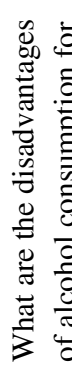 & 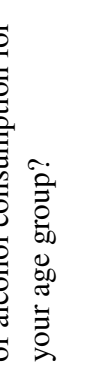 & & & & & 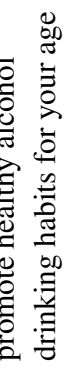 & $\stackrel{\hat{\Xi}}{0}$ & & \\
\hline
\end{tabular}




\section{CHAPTER 6}

"I am not my father": Alcohol dependence and associated stigma in Ukraine

Toornstra, A., Massar, K., Hurks, P. P. M., Kok, G., \& Curfs, L. M. G. (in preparation). "I am not my father": Alcohol dependence and associated stigma in Ukraine 


\begin{abstract}
Alcohol dependence is worldwide a severely stigmatized condition. Psychological distress linked to stigma and perceived stigma is among others related to lowered selfesteem and self-efficacy. Ukraine is in the top ten of countries with highest alcohol use worldwide. The purpose of this study was to explore (perceived) stigma in relation to alcohol use in Ukraine.

Qualitative measures consisted of 38 in-depth semi-structured interviews with adults and 81 open-ended written questionnaires with adolescents and young adults (12 - 21 years old). Multiple stakeholders participated in the interviews such as community leaders, people who recovered from alcohol dependence, and family members of people with alcohol dependence. The data were summarized following a general inductive approach. Themes that emerged from these inductions concern perceptions about development of alcohol (mis)use, labelling of people experiencing alcohol dependence, alcohol use among youth and patterns of blame, stigma by association, female alcohol dependence and consequences for offspring, stigma in official structures.

The findings show examples of public stigma in Ukraine (harmful beliefs in society based on external characteristics of an individual), stigma by association (attributing mentioned public perceptions to individuals close to the person who is subject of stigma), self-stigma (a person internalizes the harmful public perceptions), structural stigma (structures in society enable stigma happening), and provider-stigma (people in official organizations/roles in society stigmatize). Given the proven detrimental effects of stigma to health, interventions at multiple levels in society are urgently needed.
\end{abstract}

Keywords: Alcohol dependence, Ukraine, public stigma, self-stigma, stigma by association, structural stigma, provider stigma, Ukraine, norms, health beliefs. 


\section{Introduction}

Alcohol misuse is worldwide linked to a significant range of disease, death, injury and mental illness (Rehm et al., 2009). Worldwide, on average 6.2 liters are consumed per person per year, and Ukraine, with 15-25 liters of pure alcohol consumption per person per year, ranks well above this world average (WHO, 2014). Patterns of risky alcohol use in Ukraine have been associated with disadvantageous health behaviors such as early adolescent alcohol use, binge drinking, prenatal alcohol use, high risk occupations, feelings of anxiety and depression, and lowered quality of relationships and parenting (Bakhireva et al., 2011; Burlaka et al., 2017; Chambers et al., 2014; Currie et al., 2004; Polshkova, Chaban, \& Walton, 2016). Furthermore, alcohol use problems have been reported throughout the historical political changes in this region, and have been linked to mental health problems, but there is a lack of recent studies on this topic in Ukraine (Bromet et al., 2005; Wasserman, Värnik, \& Eklund, 1994, 1998). Alonso et al. (2003) found perceived stigma of $13.5 \%$ in several countries related to mental health, including Ukraine. A recent qualitative study conducted in Ukraine revealed perceptions of a normalization of daily use of wine and beer in parts of the population, and the use of and ability to digest ample amounts of strong spirits, an ability which is labeled as 'cool' (Toornstra et al., resubmitted). Paradoxically, this study also showed that alcohol dependence (AD) was considered a 'weakness' and was associated with negative perceptions of the person experiencing this dependence as well as their family members (see also Room, 2005; Schomerus et al., 2010).

Stigmatization is a social process in which differences are labelled (i.e., these human characteristics described as negative stereotypes) resulting in in- and out group classifications with loss of status for people who are stigmatized (Pescosolido \& Martin, 2015). AD has also been found to be a more stigmatized condition compared to other (mental) illness and this perception remained stable over the last two decades (Angermeyer, Matschinger, \& Schomerus, 2013; Pescosolido et al., 2010). AD for example has been associated with the perceived character flaw of a person not to be able to control oneself and with unpredictable behavior. These perceptions may serve as justification to treat people as 'being of lesser value' through overt or covert behaviors, for example by limiting the stigmatized person's access to work (Pescosolido \& Martin, 2015). Stigmatization also involves complex processes of employing power to keep people in a group, out of a group or at a distance, which tend to serve purposes of exploitation, control or exclusion (Link \& Phelan, 2014). As it is not acceptable to discriminate a person with e.g., mental illness openly power structures need to be exercised in a way that is hard to link to person(s) who stigmatize (Link \& Phelan, 2014). One person expressing a stigmatizing opinion is easy to see, and this person may become disapproved of. However, stigmatizing views expressed by a group may be accepted in parts of a society and become a descriptive or even an injunctive norm (i.e., what the public is doing and/or should be doing). Such forms of public stigma can be rather blatant and discriminatory yet when they are an integral part of society - 'everyone' treats certain groups this way - it is not often a single person behaving 
offensively, which also increases the difficulty of ameliorating such stigma. In addition to public stigma, self-stigma is defined as the situation when the subject of the described stigma believes and internalizes these perceptions (Corrigan \& Watson, 2002). The effects may differ per person, but it has often been associated with a loss of self-esteem, which may be linked to hopelessness (Mashiach-Eizenberg, Hasson-Ohayon, Yanos, Lysaker, \& Roe, 2013). In the context of AD, self-stigma has worldwide been linked to a lower selfefficacy in refusing alcoholic drinks, which might be a significant risk factor in perpetuation of the $\mathrm{AD}$, thus showing how stigma mechanisms can contribute to maintaining the status quo (Schomerus et al., 2011). Internalized stigma was identified as a barrier to seeking help and especially associated with men (Clement et al., 2015). This finding is in line with results from our previous research, in which we found that especially men in this sample are supposed not to talk about their feelings and problems (Toornstra et al., resubmitted).

Yet another recognized form of stigma is stigma by association, meaning that someone close to a person with the prejudiced condition is treated with similar prejudice, such as the children of a person with mental illness or AD (Pescosolido \& Martin, 2015). Such stigma may cause the children to be hesitant to disclose or discuss the family situation due to society's negative perceptions, which increases the chances of developing selfstigma (Burk \& Sher, 1990; Haverfield \& Theiss, 2016; Schomerus et al., 2011). Equally, partners of people with AD may akin to the person him/her self be perceived by society to be responsible for problems, while children were associated with the chance of being labelled as highly likely to develop the same problems as their parent(s) with AD (Corrigan \& Miller, 2004). Lastly, there is structural stigma, which is discrimination of a person or certain groups, such as provider-based stigma in (mental) health institutes (Pescosolido \& Martin, 2015). These different forms of stigma as seen in AD and other (mental) illness have been linked to negative effects in health outcomes such as emotional stress, reduced self-esteem, depression and/or feelings of anxiety and to the best of our knowledge have been limitedly researched in Ukraine ( Bos et al., 2013; Keyes et al., 2010).

Ukraine is one of 153 low-middle income countries, which have been identified to lack in manpower and infra-structure for mental health services (Jacob et al., 2007). Moreover, prevalence estimates of alcohol dependence and depression were higher in Ukraine compared to other European mental health survey studies (Webb et al., 2005). In men 1 out of 3 and in women 1 out of 12 were found to be heavy alcohol users. Alonso et al. (2008) measured self-reported embarrassment and perceived discrimination or unfair treatment related to one's health problems in Ukraine and concluded that this indicates high perceived stigma (Alonso et al., 2008). Given the long-lasting impact of stigmatization for the individual and their immediate environment, as well as the facts about current alcohol (mis)use in Ukraine (see also Toornstra et al., resubmitted), the aim of this research is to investigate what stigma-related perceptions exist in Ukraine about people suffering from $\mathrm{AD}$ and their family members. Knowledge about these issues may point toward directions for intervention. Furthermore, the evidence about the effects of interventions to reduce 
mental-illness related to stigma is especially scarce in low- and middle-income countries such as Ukraine (Thornicroft et al., 2016).

\section{Methods}

This qualitative study was conducted in villages and cities in (south) Western Ukraine. Semi-structured in-depth interviews were conducted with individuals from different adult stakeholder groups and open-ended questions were administered with youths, to explore perceptions about stigma. Translators were approached, and information about the study was spread by word of mouth. People from a wide range of backgrounds (i.e., ethnic-, socio-economic- and professional) were randomly invited to participate (see Table 1) and were approached via translators who spread the request for participation.

Semi-structured written open ended questions were used to explore student's perceptions of health and alcohol (mis)use. Written open-ended questions fitted in students' and schools' curricular structures. The questions concerned perceptions of health, (subjective) norms and alcohol use. Alcohol dependence (AD) in this study is not based on a medical diagnosis, but rather defined as alcohol misuse that interferes with daily functioning in family-, work- and social life and/or the need to consume alcohol. Binge drinking is defined as drinking four or five units or more on one occasion in a short period of time (for females and males respectively; Wechsler \& Kuo, 2000) and is not the same as AD. Alcohol misuse in this study is defined as any alcohol use that forms a risk to health, such as binge drinking and underage alcohol use.

\section{Participants $^{3}$}

The study sample consisted of people of multiple ethnic backgrounds, i.e., Ukrainian, Hungarian, Russian, and some Roma and Ruthenean residents. Interviews were administered with 38 participants aged $20-60+$ years old, and 81 questionnaires with students 12 - 21 years old. The participant categories (see Table 1) emerged from the word of mouth approach and the data (i.e., self-reported health workers, family of people misusing alcohol, AD survivors).

${ }^{3}$ Please note that data collected among this sample as part of a separate study are reported elsewhere (Toornstra et al., resubmitted, see chapter 5). 
Chapter 6

TABLE 1. SAMPLE COMPOSITION (NOTE THIS TABLE CONTAINS FREQUENCIES BASED ON SELF-REPORT

\begin{tabular}{|c|c|c|c|}
\hline & RURAL & URBAN & AGE \\
\hline \multicolumn{4}{|l|}{ INTERVIEWS } \\
\hline PEOPLE RECOVERED FROM ALCOHOL MISUSE & 3 & 7 & $18-60+$ \\
\hline MALE / FEMALE & 6 & 4 & \\
\hline PEOPLE AT HIGH RISK OF CONTINUED ALCOHOL MISUSE & 1 & 6 & $30-55$ \\
\hline MALE / FEMALE & 6 & 1 & \\
\hline FAMILY OF PEOPLE MISUSING ALCOHOL & 3 & 2 & $20-39$ \\
\hline MALE / FEMALE & 0 & 5 & \\
\hline COMMUNITY LEADERS & 3 & 3 & $30-65^{*}$ \\
\hline MALE / FEMALE & 4 & 2 & \\
\hline HEALTH CARE PROFESSIONALS & 2 & 3 & $25-59$ \\
\hline MALE / FEMALE & 4 & 1 & \\
\hline INTERNATIONAL VOLUNTEERS \& PROFESSIONALS & 5 & 0 & $15-60+$ \\
\hline MALE / FEMALE & 1 & 4 & \\
\hline \multicolumn{4}{|l|}{ OPEN-ENDED QUESTIONS } \\
\hline 1 MIDDLE SCHOOL & 1 & & $12-21$ \\
\hline MALE / FEMALE & 9 & 8 & \\
\hline 1 HIGH SCHOOL & 1 & & \\
\hline MALE / FEMALE & 10 & 16 & \\
\hline 1 MIDDLE AND HIGH SCHOOL & & 1 & \\
\hline MALE / FEMALE & 9 & 15 & \\
\hline 1 COLLEGE & & 1 & \\
\hline MALE / FEMALE & 6 & 8 & \\
\hline
\end{tabular}




\section{Materials and procedure}

The first researcher, assisted by translators, approached schools, colleges, and rehabilitation centers, and individuals within these settings. Some participants were asked from a selection of the population; people, who self-reported to have overcome alcohol misuse and/or dependence. Word of mouth via translators asking for people who might be interested in communities was the primary strategy, which resulted in people who gave permission to be contacted. The translator and researcher subsequently gave information and arranged interviews in case of consent. The research ethics committee of the Faculty of Psychology and Neuroscience of Maastricht University, The Netherlands, approved this study. Appropriate levels of informed consent were obtained from all participants. No compensations were involved. Respondents stated to be from a wide range of educational backgrounds; ranging from the minimum number of years of completed compulsory education to medical doctor degrees.

The in-depth semi-structured interviews contained questions about alcohol (mis)use in Ukraine, its perceived relation to health, and participant's perceptions regarding AD. Each interview lasted about one to one and a half hours. The introduction and questions were translated, and further explanations were asked or probed during interviews in case of simultaneous translation. Interviews were carried out in a room with only the interviewee, translator and researcher present. The questions were translated, and this written copy was at the start of the interview given to participants to inform them about the content of the questions and provide the chance to ask for changes. The interviewer introduced the topic and asked the first question, which was simultaneously translated. The written questions were only a guide and were little used by respondents. Participants started to tell their stories after this verbal introduction. All interviews were audio-recorded and transcribed.

Next, middle-, high school and college students of institutes that had given consent were asked to complete short semi-structured written open-ended questions. Ten questions covered students' views of their own health, healthy legislation of alcohol use in relation to their age, (subjective) norms, peers and alcohol consumption and prevention of alcohol misuse. This was based on the theory of reasoned action approach in which behavioral beliefs, control beliefs and normative beliefs are thought to influence intentions, which in turn affect behaviors (Fishbein \& Ajzen, 2010). All students present on the day completed the open-ended questions, except for those not present. Respondents only participated in one role, e.g., a teacher was approached as teacher only and not in case of self-report as an AD survivor as well.

\section{Data analysis}

The interviews and semi-structured open-ended questions (questionnaires) were analyzed by means of a general inductive approach; that is rigorous rereading supported 


\section{Chapter 6}

grouping of subthemes into the main themes that emerged from the data (Thomas, 2003). A second researcher independently coded two interviews and ten student questionnaires. In case of disagreement of more than $20 \%$ on themes, a third independent coder was consulted. Nvivo 11 was used for coding and finding the leading themes.

\section{Results}

The following themes in relation to alcohol use and stigma emerged from the data.

Perceptions about development of alcohol (mis)use

Alcohol consumption by children in public was described as unfavorable, but also as something about which little could be done. A male community leader ( 35 years old) described a working day:

"Everyone knows about that but no one can do anything against. ... Because I was asking: Have you been drinking today? [The boys replied] No, no. Community Leader] But I can see! [Boys] Well just a glass of wine.”

He furthermore reported that the boys he described above had consumed alcohol for years, so they probably started when they were about ten years old. Three AD survivors reported they started to consume alcohol regularly between 12-14 years old. Increases in quantities and percentage of alcohol were described by multiple stakeholders and increased tolerance was seen and labeled as a sign of 'strength'. A male AD survivor (66 years old) described how as a child, he helped his father who was in charge of wine in the Kolchoz (i.e., a collective farm under communism):

"I became a strong child very early. By the time I was 16 or 17 I wasn't drunk after a liter of wine. ... after a few years ... I could drink half a liter of vodka or palinka ... and no one could notice that I drank anything because I wasn't drunk."

Other AD survivors, some youths, and community leaders also reported this perception (either about self or observed in others) that consuming copious amounts of alcohol was a sign of 'strength'. The reported norms included consumption before, during and after work and descriptions included a focus on group values of being colleagues, friends, a culture of hospitality and availability of (unrecorded, i.e., self-produced) alcohol to a person of any age (Toornstra et al., resubmitted). A male community leader (35 years old) described his observations that alcohol which is on the table needs to be finished irrespective of the amount, which he felt is shown in sayings such as:

“One cannot stand on one leg.” 
However, a male survivor (44 years old) described the need to consume all available alcohol as a characteristic of addiction:

"... [for an alcoholic] there is no enough ... he lost control ... If there is only one glass of alcohol than that is enough, or if there is one liter of alcohol than that is enough.... I had to look for it by the time I got alcoholic, and the people did not offer me alcohol ... It made me feel like I am alcoholic, and I do not belong to the society."

A male health worker (55 years old) stated that in his opinion AD can affect anyone:

"It doesn't matter what nationality you come from; It can be a Gypsy child, Hungarian child, Ukrainian, Rumanian or Russian child; it doesn't matter.”

\section{Labelling of people experiencing $A D$}

The AD survivor above stated when he started to realize that he was dependent that he did 'not belong' to his group of colleagues and friends anymore. He described how he had consumed alcohol on a regular basis with his colleagues, but since experiencing AD he found himself 'searching' for alcohol, needing it rather than enjoying it. In addition to $\mathrm{AD}$ being described as making him feel like an outsider, it was labeled by other respondents as a 'weakness', and the person suffering from AD was openly blamed for their behavior. A female community leader (35 years old) reported:

"In this society it is generally accepted that people look down on alcoholics. Because they consider them to be weak. They consider them to be lazy."

A female international community volunteer (20 years old) reported that $\mathrm{AD}$ was described by other community members as:

"He does not want to work. He drinks. ... of course it is his fault."

Another female international community volunteer (19 years old) reported that she heard people using these labels in the community even after the AD survivor had abstained from alcohol for years:

"And this pressure I think is even making it harder to leave it. Because even if you've not been drunk or something for quite a long time, they still have this image of 'Oh, he is an alcoholic'.... They don't forget it."

Job loss was reportedly linked to alcohol use. Interestingly, the community also tried to look after these unemployed individuals: Family members of people with AD and community leaders reported about the allocation of varying jobs to people with $\mathrm{AD}$, such as 


\section{Chapter 6}

the pastor who asked an employer to give a mechanic 'another chance'. Another female family member of a person experiencing AD (female, 35 years old) reported her husband was given the community cow-heard job, which requires responsibility due to every family's need to have a cow. However, another male community leader expressed surprise that these local job offers are not always accepted by the individuals suffering from AD.

In general, there was much agreement among respondents about individuals who were addicted to alcohol. The word genetics was mentioned, but hardly elaborated on. There were only a few individuals (e.g., a health worker) who communicated different perceptions. One female community leader (35 years old) stated her different opinion about AD:

"10\% genetics, $60 \%$ socialization and $30 \%$ life happenings".

A female family member ( 25 years old) reported she opposed the labelling of people suffering from $\mathrm{AD}$ as being lazy and indicated that in general, Ukrainian people tend not to see addiction as a disease that needs treatment:

'[In Ukraine people say] 'Everyone is tired when doing heavy work.' It is a different way of looking at things. Only physical illness is illness. Depression or something is not an illness. That is why there is such a high percentage of suicide."

\section{Alcohol use among youth: Patterns of blame}

Alcohol use was reported by youths and AD survivors as a way to socialize with peers, and initially linked to feelings of happiness or the need to forget problems. However, alcohol use was also related to the experience of negative emotions (see also Toornstra et al., resubmitted). When asked about the reasons for alcohol use starting at a young age, respondents allocated blame for excessive alcohol use and alcohol dependence to youths themselves, peers (social influence) and to parents (social learning, or a lack of parenting skills). One female student's statement represented the responses of several other students that it is a young person's own responsibility (15 years old):

"Well, there are a lot of youngsters aged 12-14 who try to drink very early, but this is their decision, everyone chooses his fate himself."

In line with the perceptions of adult $\mathrm{AD}$ reported above, children who consume alcohol were described as choosing this themselves. Others implied a character flaw or moral failure but were not clear on which morals would become lost due to alcohol use or vice versa. A female college student (20 years old) reported:

"I think, these young people poison their body, and last but not least, they lose their moral values as well.” 
Some youths blamed adults in general, including teachers, for the development of $\mathrm{AD}$ among young people. About one third of youths in the open-ended questions reported negligent parenting as one of the causes of young people turning to alcohol and eventually ending up dependent. A female high school student (17 years old) said:

"It depends on parents. If they are strict enough, and take care of their children, they won't be alcoholics."

A female AD survivor reported how she as a young adolescent went out with her older sibling (and thus her sibling's peer group) and her parents failing to notice her alcohol consumption that would result in her bedroom smelling of alcohol the next morning. Some students in the open-ended questions openly blamed the peer group for causing both the initiation and the continuation of alcohol use. Here patterns of social influence or peer pressure were also evident. For example, a male college student (20 years old) stated:

“I think, most youngsters follow their peer's example, and not their parents."

\section{Stigma by association}

Part of the students in the open-ended questions reported making plans and setting goals for higher education and expressed not wanting to develop alcohol use problems. However, for some children this is hard, since the respondents also mention public labelling of children in school due to parental AD. A female family member (25 years old) whose father supposedly experienced $\mathrm{AD}$ reported:

“... that teacher saw my last name and said, "Oh that is from that alcoholic ... they do not look at children as an independent person who has chances ... they have an image of parents ... oh nothing good will come from that child."

When prompted how this made her feel, she reported "crying”, but she told how she sought other support in her local community. She reported on her observations of the consequences of this labelling by teachers for herself and her peers with AD in the family. She told how this created self-labelling as well, which in turn affected their choices and their futures. She felt all of them, including herself, were not the strongest candidates for university, but she studied hard and achieved her goal and secured funding. Furthermore, she reported discussing with her brother that everyone has a choice and she stated that she concluded herself:

... "they had put crosses on themselves ... I, for myself had not put a cross on myself. ... "I am not my father." 
The fear of following in one's father's alcoholic footsteps was a common theme among the respondents. Statements were made by international professionals about community members, and by AD survivors that they overcame their addiction and/or did not become alcohol dependent 'like their father'. One male community leader described how a teacher explained to that him due to his father's AD, he should not ever try alcohol, which he reportedly took to heart. Two older male AD survivors reported each about a son of theirs who also developed AD. Several of these reports mentioned another son who did not develop problems with alcohol. Interestingly, these latter children were described or selfreported as having had different contacts in their community when they were a child. They were exposed for example to an adult role model, family friend, or other community networks where alcohol consumption was not advocated.

Intergenerational patterns of alcohol misuse or dependence involved both males and females and were reported by three respondents. The idea also existed among respondents that once the parents were alcohol dependent, their children would also become alcohol dependent, and hinted toward a heritable component of AD. However, a health worker stated that children who were placed in families due to the AD of their biological parents, were mostly doing well if they were placed in a responsible family that did not allow them to consume alcohol up till 18 years old. He stated (45 years old):

“... I suppose that this could be included in their genes. But so far since I have been working in this system I haven't seen any children till the age of 18 who would have serious alcohol problems. ... And in my experience if a child gets attention and love and proper care and then he does well at school and after that going out to the individual life they can be fully grown individuals in all means and they can lead a normal life if their parents are responsible; if the family they got to is responsible and takes care of them well."

Multiple sources reported that if the father in a family suffered from AD the mother might consciously not consume alcohol and take care of the children and/or husband. Reportedly, this took a toll on such women - and the entire family - illustrated by a female family member ( 25 years old) who described how her mother behaved in response to her father's reported $\mathrm{AD}$ :

"She always took good care of him. He never left the house with dirty clothes. ... I think my mother must have thought more 'I do not want to live anymore'. Because my mother is also very aggressive especially when my father was drunk. She could not stop herself. ... But she was screaming the whole night. And in our street, we had more families with such problems. If it was quiet in our house it was drama in the neighbor's house." 
Female $A D$ and consequences for offspring

Although the interviewees reported mainly about male $\mathrm{AD}$, there were rumors about females experiencing $\mathrm{AD}$, as reported twice by a community leader and an international volunteer. A female health worker (32 years old) reported that in her perception at work in a local hospital, alcohol misuse was a problem for both genders. She also observed female mental health problems, sometimes as a result of having to take care of a husband with AD:

"There are special rules in the department where the ill people with liver problems are cured. And there is a doctor who specially deals with these liver problems. But this is difficult to tell if these ladies are there because of alcohol problems, only if you can see like that their belly is bigger that is when you can estimate they have alcohol problems because their liver is big. ... There were two ladies in the internal medicine department. One of them was an alcoholic and one of them tried to commit suicide... This lady's husband was an alcoholic. She [tried ... ] committed suicide because of her husband, because of her sorrow."

One AD survivor reported that his mother committed suicide, reportedly linked to AD. Females were reported, similarly to males, labelled as failing in their responsibilities if they were alcohol dependent. A female community leader (35 years old) reported that the community would perceive a female with AD:

“... not only be a burden to her family but to society as well.”

Overt and covert female alcohol misuse was reported. The latter was linked by an $\mathrm{AD}$ survivor to men travelling abroad for work and women facing daily life alone, having a drink with female friends and not realizing that they were becoming dependent. A female health worker described having heard of a female "drinking group". Additionally, several sources described alcohol consumption in relation to partner dynamics, including alcohol use in pregnancy. A female AD survivor (55 years old) reported:

"And I tried to solve my problems with drinking alcohol but of course it wasn't the solution to drink alcohol. Then I got acquainted with my husband. So we lived together without getting married and we had two girls soon. But meanwhile we were drinking alcohol. Not just me, but the two of us together. And you see this house, we lived in this single room. It was a half ready house, because we didn't pay attention to it."

Alcohol use in pregnancy was reported by multiple sources and linked by AD survivors to health problems in children, dependence in infancy and possibly other problems later in life. A female AD survivor (20 years old) described it as:

“... to kill a new personality, a new life, a human.” 


\section{Chapter 6}

She then emphasized that more women should become aware of the risks of alcohol use in pregnancy:

“... we should not let that happen, everyone should know that all that's consumed by the mother is also consumed by the baby, if you want the child to be born healthy, you must hold on however hard you wish."

Two male community leaders reported that for some women in the community, having babies is "business" due to the government grant, which is mostly paid as a lump sum at birth. A male community leader reported (35 years old):

"But family; when I have one child, governments pay [short silence thinking] for example 40,000 Hrivna [local currency]. When two 65,000. When third 100,000 Hrivna. And for people who don't like work, but like government money, children, ehm, more children it's money. And sometimes it's more business, born children."

Reportedly, children born to mothers (and fathers) with AD were abandoned or placed in state care. Several community leaders and a health worker reported that this happens quite often. A male community leader reported (35 years old):

“... in our... region, children from three years old till twelve, we have about ... five or seven orphanages. And in every orphanage more than hundred children. [...] In the Ukraine [we] have a lot of such orphanages. Mostly it is from parents who have problems with alcohol."

About half of youths reported in the open-ended questions that they would support prevention of alcohol problems in general through knowledge (education) and/or fear appeals and/or changing the 'happy image' related to alcohol consumption which they reported was conveyed on TV.

\section{Stigma in official structures}

Family and AD survivors reported little governmental interest in AD. Moreover, youth stated that in their view that the government should act on underage access to alcohol. In the questionnaires a response was asked to the research finding that worldwide the age of first alcohol use is 12 despite most laws forbidding alcohol use up till 18 years old. Multiple responses were similar to this in reply (male, 20 years old):

"I know shops, where anybody can buy alcoholic drinks. It is a big problem in our country. Many people break the law." 
Several respondents appeared to convey a sense of disappointment in their government and reported that even if the government had figures on alcohol misuse they would be the communist way, meaning adapted to the perceived need of the official's request, like in communist times. In general, there is an absence of figures on the prevalence of AD in Ukraine and three male health workers (35-55 years old), stated this was because:

“... the government does not want to know about this problem."

In addition, the quality and quantity of mental health care available to those suffering from $\mathrm{AD}$ was reported to be severely lacking as well. A male community leader (35 years old) reported:

"Biggest problem in our country that we do not have doctors, ... People who can help that alcoholics in a hospital for example; especially we have very big lack of psychiatrist, or what is, people who can help, can talk to the alcoholic."

Medical care was described by many respondents as a state system accessible for all. In contrast however, several community leaders and health workers reported that people need to pay for medical care. A community leader (male, 35 years old) stated that often individuals suffering from $\mathrm{AD}$ were refused care, presumably because they would not be able to afford this:

"And in our hospitals, they must pay for medicine, for everything, we must pay, but they do not have money. Alcoholics don't have money for rehabilitation, for healing. And doctors also when they see alcoholics, they just: no, go home."

A female health worker (32 years old) in another city reported her observation of prejudice against people admitted to the psychiatry department of hospital, and the lack of trust of patients in doctors, she consequently also perceived. She stated:

"Because the staff of the hospital calls the psychiatry the crazy hospital. Those who get to the psychiatry they already lay their hands off them. But a lot of people who are in their hospital should be in psychiatry. If the doctor and the patient could talk sincerely with each other than the science of medicine would not be at this level. My experience is that the patients don't trust the doctors."

\section{Discussion}

Alcohol dependence (AD) has been linked to stigma (Schomerus et al., 2010), and this stigma may aggravate the psychological, physical, and social harmful consequences of 
the dependence itself. Additionally, alcohol dependent individuals are believed to be one of the most severely stigmatized groups in society (Schomerus et al., 2010). Given the omnipresence of alcohol described in the history and culture of Ukraine (WHO, 2014, 2018; Toornstra et al., resubmitted), the question arises what constitutes to perceptions about $\mathrm{AD}$ and to what extent people suffering $\mathrm{AD}$ experience such stigma; and how this in turn affects their access to (mental) healthcare. The purpose of the current study was to investigate the perceptions of stigma associated with $\mathrm{AD}$ also in relation to family members of people with $\mathrm{AD}$.

$\mathrm{AD}$ was in this sample reported as a public stigma (i.e., significant parts of a society believe a stereotype; Corrigan \& Shapiro, 2010), and also appeared to be heavily linked to perspectives on economic values of individuals. These perceptions became evident from statements which described both male and female AD sufferers as 'lazy' and 'failing in their main duties' - whether this was in providing income for their families, or in parenting and childcare. Stigma generally serves purposes of exploitation, domination, exclusion or norm reinforcement of the in-group (Crocker \& Major, 1989; Link \& Phelan, 2014). In the current sample, marginalization of individuals suffering from AD was repeatedly reported, both by AD survivors ('not belonging') and by family members. Furthermore, the condition of AD was causally perceived in this sample as an internal personal attribute of having lost control, which became evident from statements about individuals being weak; and the application of the stigmatizing mechanism of failing to take responsibility (Bos et al., 2013). The respondents purported an image of the 'lazy' male with $\mathrm{AD}$ who does not have a job, or the female with AD who does not fulfil the expected role as a mother. These perceptions are evidence of a view of $\mathrm{AD}$ as being voluntary in nature; one is not medically ill but more or less choses to become dependent on alcohol (Phelan, Link, \& Dovidio, 2008). The locus of control in case of AD is thus strongly deemed to be within the person, whose perceived failure is met with anger and (perceived) exclusion from society (Dijker \& Koomen, 2003).

Indeed, in the current sample, AD appeared not to be seen as a mental illness or as the result of environmental risk factors, limitations in skills to address life's challenges, and genetic susceptibility (Sigvardsson, Bohman, \& Cloninger, 1996), but rather as a choice to violate a norm, of not being able to deal with group habits, or to resist peer pressure. That appeared the explanation to perceive AD in Ukraine as a weakness. In Ukraine there is a long and cultural tradition of drinking alcohol and being able to consume large quantities in one session (binge drinking); the latter is considered to be a sign of being a 'strong' person (see Toornstra et al., resubmitted). This is in line with research by Room (2005) who described alcohol use as a social behavior that is associated with an individual's status in a group. Additionally, in the current sample there were reports of early onset-alcohol use; often in childhood such as sipping wine during family meals. Jackson, Ennett, Dickinson and Bowling (2013) associated alcohol use with lower feelings of academic competence and noted that differences with abstinent peers (in learning and behavior) start in middle childhood. It became evident from the reports of youths in the open-ended questions that stigmatization also appears during this time (again associated with behaviors that were 
perceived to be related to alcohol use and cause problems in school and may affect others, i.e., the 'strength' versus dependence described in this sample).

In the current sample, the consequences of $\mathrm{AD}$ were mainly described in terms of job loss and poverty. Continued political-economic transition issues were described by the respondents such as a lack of employment opportunities and financial uncertainty since Ukraine's independence (see also Hellman, Jones, \& Kaufmann, 2003). Ukraine was described by a participant in this sample as characterized by 'the absence of a middle class' and the economic resources are thus concentrated in the hands of a small group of upper class individuals. This creates competition for jobs among the lower-class individuals. One reason for stigmatizing individuals suffering from AD may thus be to reduce the competition by preventing 'weak' people to participate in the labor force. Structural stigma furthermore prevents these individuals from accessing the medical and mental health care they need. Phelan, Link, and Tehranifar (2010) propose in their Fundamental Cause Theory that resources play a crucial role in creating and maintaining social conditions and health inequalities, i.e., individuals with higher SES have a set of flexible resources to their availability (knowledge, power, money, prestige, beneficial connections) that allow them to avoid or counter multiple disease (outcomes) compared to lower SES. Indeed, Hatzenbuehler, Phelan and Link (2013, p. 813) define stigma 'as the co-occurrence of labeling, stereotyping, separation, status loss, and discrimination in a context in which power is exercised'.

Stigma by association, that is, viewing family members of people with AD as having similar characteristics as the person with $\mathrm{AD}$, was also repeatedly reported in this sample. The respondents talked about how blame for example, for the onset and relapse of the condition was regularly attributed to the spouse in this Ukrainian sample. There were reports of attempted suicide by wives or nightly fights in families where at least one person experienced AD (see also Schomerus et al., 2010) and some respondents hinted at intimate partner violence, which has also been linked to alcohol use. However, to identify whether such issues are indeed linked to alcohol misuse, longitudinal studies to clarify behavioral patterns (variables and the direction of their effect) in Ukraine need to be conducted (Devries et al., 2014). Children of individuals with AD were in this sample more associated with stigma through contamination, that is children may be perceived to be contaminated by their parent's condition such as AD or mental illness (see also Corrigan \& Miller, 2004). This form of association with parental AD was also evident in the reports that teachers applied certain labels to pupils (see also Crisp, Gelder, Goddard, \& Meltzer, 2005; Pescosolido \& Boyer, 2010) as well as the reports by youths who described children who consume alcohol as exhibiting behavioral problems and disturbing the general learning processes in class. Additionally, part of Ukrainian youths allocated blame for child alcohol consumption to these children's parents (that is stigma by association), and in some incidences to the children themselves. Some youths also reported they would only socialize with a group of peers that did not consume alcohol. Similar findings were reported by Crisp et al. (2005), who found the most negative opinions about mental illness among 16 - 19 years old. Equally, Burk and Sher (1990) found that peers with stigmatized parents were 
perceived more negatively compared to peers whose parents did not abuse alcohol or experienced mental illness. These forms of stigma carry the risk for children of parents suffering from $\mathrm{AD}$ to develop self-stigma.

Self-stigma, that is the internalizing of the beliefs the public holds about AD - the 'cross' that teachers and peers put on individuals from families where one person suffers from $\mathrm{AD}$ - was also found in this sample. For example, the daughter not accepting the negative labelling by teachers of herself, linked to her father's AD, and her refusal to put the 'cross' on herself. Similarly, there were reports of pupils not being able to plan their educational future, for example being labeled by teachers as 'one of those' (i.e., a child of a parent experiencing AD) that was 'not good enough' for university. The psychological distress associated with self-stigma was thus described in this sample as not perceiving a child as an individual human being with their own chances for a future (see also Mak \& Cheung, 2010). The issue of self-stigma among children from families in which one or more individuals suffer from $\mathrm{AD}$, is complicated by the fact that in Ukrainian society alcohol can be easily obtained and drinking is part of family and society traditions (see also Donovan \& Molina, 2011; Toornstra et al., resubmitted). Multiple respondents in this sample, but especially AD survivors, reported they had established their habit of regular alcohol use outside the family-home between 12-14 years old. Additionally, youth reported significant underage alcohol use. Furthermore, it was reported that 'everyone knew' about underage alcohol use in the community. On the other hand, the fact that youth in the openended questions overwhelmingly claimed that they did not want to touch alcohol before the age of 18 years old, suggests there may be a hesitation to openly report on and discuss (underage) alcohol use, which carries the risk of sustaining the marginalization of individuals with AD.

Societal disapproval appeared strongest for females suffering from AD, especially related to drinking during pregnancy and the consequences of child impairment, as well as the risk of child neglect and abandonment. Simultaneously, 'hidden' female alcohol use was noted. AD in women is an invisible- and/or stigmatized condition, which poses a barrier to treatment both for providers of support and the women maintaining their covert status (Mittal, Sullivan, Chekuri, Allee, \& Corrigan, 2012). On the other hand, keeping female alcohol use hidden however, may shield the women from societal disapproval and may help them to secure basic care for the family e.g., clothes and food hand-outs.

In addition to public stigma and stigma by association, structural stigma was reported in several forms in this sample (see also Pescosolido \& Martin, 2015) and it clearly illustrated how stigma in its various forms functions as a barrier for access to care. For example, there was mention of insufficient or outdated services to individuals suffering from AD. The focus was on the physical symptoms of dependence and some respondents mentioned a lack of governmental interest to quantify the health problem of $\mathrm{AD}$, and doctors' refusal to treat AD suffers because they would be unable to pay for the health services. Last, the described nature of communication about people experiencing AD implies provider-based stigma (see also Pescosolido \& Martin, 2015); psychiatric medical care was labeled by hospital staff as something for the 'crazy' people. Other psychological 
support was reported as insufficient in quantity and reports of successful recovery were mainly related to a few private rehabilitation centers.

\section{Limitations}

This qualitative study originally aimed to investigate general perceptions of alcohol (mis)use among survivors of AD and other stakeholders, and although the theme of stigma in its various forms was not specifically targeted, it clearly arose from the data. Subsequent research should therefore focus on a more in-depth investigation to verify and to clarify the perceptions on the different forms of stigma. For example, multiple family members experiencing $\mathrm{AD}$ and/or intergenerational $\mathrm{AD}$ were described by some respondents and this was specifically associated with groups of houses or flats (geographical locations) where multiple families lived. This description gives rise to the question whether there is a concentration in locations of people experiencing $\mathrm{AD}$ and what mechanisms surround a potential segregation. Moreover, from the current data it is not clear which emotions or attitudes are the main drivers of stigmatization, i.e., whether public fear or anger are the main drivers of stigmatization and avoidance (Bos, Schaalma, \& Pryor, 2008). Furthermore, our recruitment approach may have caused a sampling bias. We gauged interest in participation through word of mouth started by translators since this type of communication patterns may come close to natural communication patterns in the participating communities. Moreover, we were careful to assure that the respondents from institutions and organizations came from a balanced mix of public and private settings.

Last, in addition to alcohol use and problems surrounding AD, multiple interviewees reported that home-made drugs are overtaking alcohol problems in quantity, severity, and mortality. Currently it is not known what the consequences of such drug use are in terms of stigmatization, and whether the stigma associated with drug use interacts with the alcohol misuse stigma or contributes to layered stigma (Earnshaw, Smith, Cunningham, \& Copenhaver, 2015; Stutterheim et al., 2016). Thus, further research is needed to investigate the interrelationships between these kinds of substance abuse, how they in turn influence stigmatized reactions in the communities, and what the psychological and health-related consequences are for the individuals and their families.

\section{Implications for practice}

Stigma has been causally implicated in health problems, as well as accessibility to healthcare. The structural components that need to be addressed are public perceptions as well as the conditions in society that give rise to stereotypical beliefs about the people subjected to stigma (Weiss, Ramakrishna, \& Somma, 2006). Research has shown that it is possible to achieve a reduction in individuals' stigmatizing attitudes and behaviors by promoting interpersonal contact between people in society with those in the stigmatized 
condition (Corrigan \& Kosyluk, 2013, 2014). However, evidence shows that changing a society's stigmatizing beliefs is complex and may need intervention at political and economic levels (Bos et al., 2008). Phelan et al. (2010) propose in line with Fundamental Cause Theory to pursue the multiple goals of improving overall population health while at the same time reducing health inequalities. Thus, all policies that contribute to a redistribution of resources such as housing for the homeless, social security, and health care for all, are posed to contribute to reducing health inequalities. A simultaneous approach of reducing risks for at risk groups would in the context of Ukraine most likely have to focus on reducing the availability of alcohol and its accessibility for all ages. Furthermore, in line with Fundamental Cause Theory based on SES inequalities, it is recommended to implement school-based interventions to increase knowledge about the health consequences of (early) alcohol use as well as the lived experiences of AD sufferers. Policy to develop such programs inherently involves the need for research and thus governmental attention for $\mathrm{AD}$ sufferers and their resource problems.

Provider-based stigma by professionals in various institutions requires - in addition to increasing knowledge about mental health in general and addiction in particular - specific professional training, such as enhancing interpersonal contact, increasing empathy, and various forms of cooperative problem solving (Batson \& Ahmad, 2009; Paluck \& Green, 2009). In line with this and the principles of Fundamental Cause Theory, the focus should be on reducing a medical model approach to development and learning. This approach is deterministic and views problems to be a constant within a person (e.g., a child) and as such not changeable. A cooperative learning environment in line with varying student learning needs requires training of teachers and allocation of additional resources as agreed worldwide in the rights and disabilities act of children (Lundy, McEvoy, \& Byrne, 2011; UNESCO, 1994).

Importantly, appropriate services are needed for people subject to stigma, and in particular an increase is necessary in the number of mental health services (quantity) without provider-based stigma (quality). Reported mental health problems in the current sample appeared to range from low self-esteem and/or self-efficacy (and associated risk behaviors) to depression, anxiety, mood disorders, and (attempted) suicide. Appropriateness of services may concern specialized approaches and researching effective treatment for different forms of mental illness versus general psychotherapy. Stigmatizing has been found to be a barrier to access treatment, engage in treatment and adherence to treatment (Mittal et al., 2012). Motivational Interviewing (MI; Miller \& Rollick, 2002), a personcentered method grounded in Self Determination Theory, has been found to facilitate change, to increase treatment engagement and positively affect treatment outcome for example in mental health problems, and substance use in adolescents (Romano \& Peters, 2015). One core principle is compassion by not judging but reflecting the interviewee's reports back to them for consideration. It is predominantly used as a complement to other treatment such as medical care, life style related interventions or Cognitive Behavioral Therapy (CBT; Britton, Patick, Wenzel, \& Williams, 2011; Jensen et al., 2011; Lundahl et al., 2013). Notably, fear appraisals focusing on the negative consequences of alcohol use - 
as were proposed by multiple sources in this sample, need to be placed in the context of evidence and theory. AD survivors reported their lengthy denials, indicating people know the threat to health but struggle to understand that it applies to themselves, and may be hesitant to accept self-relevant threatening health messages (see e.g., Milne, Sheeran \& Orbell, 2000). Rather, increasing openness to such 'undesired' messages concerning risky health behavior should be the focus of intervention developers, for example through employing self-affirmation (e.g., a component of motivational interviewing; Miller \& Rollnick, 2002). Moreover, providing coping information in addition to the health threat, e.g., where to go for help is recommended (Ruiter, Kessels, Peters \& Kok, 2014).

Summarizing, programs that enhance autonomous self-regulation (Deci \& Ryan, 2008), increase self-efficacy, self-compassion and empathy may alter behavior and norms, especially if started from an early age in schools. Masten (2011) highlighted the worldwide urgency (due to environmental events) of building resilience in children in developmentally appropriate phases. Especially, values such as human interdependence (differently shaped per culture) were associated with resilience in youth (Theron, Theron, \& Malindi, 2013). Enhancing competence in for example coping skills but also self-confidence, was found to work in a preventive manner and to have a cascading effect, thus increasing the chances of healthy development (Masten et al., 2004). Peer counseling was included in Masten's (2011) resilience framework, in which children in the current sample who have chosen not to be 'like their father' (with AD), may be able to share their experiences. 
Chapter 6 


\section{CHAPTER 7}

General Discussion 


\section{General Discussion}

Learning from early school ages is important for a range of life outcomes (Hughes, 2011). The aim of the five studies described in this thesis was to investigate the care for schoolchildren in Western Ukraine. Competencies in advantageous academic learning and life outcomes (e.g., employability) have been linked to a group of cognitive functions called executive functions. Measuring these functions requires first valid measurements. The first objective of this dissertation was therefore to collect data to establish demographically corrected norms for tests measuring executive functions and underlying cognitive functions in a sample of schoolchildren in West Ukraine. The second objective of this thesis was to investigate perceptions of Ukrainian adolescents and their families, communities and society on health and more specifically alcohol misuse in West Ukraine. First, the chapters 2-4 are summarized and discussed (aim 1) followed by the investigation into perceptions into health and alcohol (mis)use which was aim 2 of this thesis (chapters $5 \& 6$ ). The last part of this general discussion concerns considerations about limitations of the studies in this thesis as well as implications of the results and recommendations for future research.

Aim 1: Establishing norm scores for tests measuring executive (and underlying cognitive) functions among Ukrainian schoolchildren (chapters 2-4).

Executive functions (EFs) have been associated with success in learning (reading and mathematics; Geary, 2013), which in turn has been associated with beneficial life outcomes such as obtaining and keeping a job (Duncan et al., 2007; Parsons \& Bynner, 1997). EFs have been defined as a set of closely related yet independent cognitive abilities such as attention, cognitive flexibility and goal setting, that allow one to plan and to execute the steps needed to achieve a goal (Hughes, 2011; Anderson, 2002). Development of cognitive abilities in general and EFs specifically has been linked to cultural influences, i.e., one's cultural background influences how one thinks, perceives information and makes decisions (Ardila, 1995, 2005). Culture is thought to comprise values, beliefs, knowledge, and norms in a society. Cognition is therefore thought to vary across different cultures (Ardila, 1995), which makes it important to investigate the developmental trajectories of EFs in different populations. Therefore, in order to assess whether Ukrainian children's development is within the normal range, one needs to collect reference data on how Ukrainian children normally perform on tests of cognitive functions underlying EFs (i.e., short-term memory (STM)) and EFs tests. Unfortunately, to the best of our knowledge, reference data for tests measuring EFs in Ukrainian children are scarce. In the chapters 2, 3, and 4 data were collected for seven tests measuring EFs and underlying cognitive functions. In these three chapters a novel, promising approach called continuous norming or regression-based norming was applied (in line with e.g., Van der Elst et al., 2011). In this approach, relevant demographic characteristics were included, such as age, sex, and/or level of parental education, as main predictors in the regression-based models, since in the data (chapter 2-4) an association between these factors and EF (and underlying cognitive 
functions, e.g., STM) performance was found. These found associations were in line with other studies linking cognitive test performances with e.g., age, sex, and/or level of parental education (LPE; Lezak et al., 2012; Taylor \& Heaton, 2002). For instance, short-term and working memory abilities have been associated with age, i.e., the older children performed better on tests measuring these cognitive abilities compared to younger children (Alloway et al., 2006). Indeed, executive - (and underlying cognitive) functions were found to be less refined in younger children and improve during development in efficiency and complexity toward a highly specialized system (Alloway et al., 2006; Anderson, 2002; KarmiloffSmith, Scerif, \& Ansari, 2003). Another example, Voyer and colleagues (2017) found a small yet significant effect of sex in their review of visual spatial short-term memory: i.e., boys outperformed girls on tests measuring this function. Last, level of parental education (LPE) has been described as an estimate of socio-economic status (Luria et al., 2009); higher levels of both of these constructs have been linked to higher scores on cognitive functions such as STM. The three demographic variables of age, sex and LPE were therefore included in this dissertation when collecting data to establish norms for schoolchildren in Ukraine. However, the data also showed that not all demographic variables had an influence and in addition, different combinations of demographic variables as predictors were observed, e.g., age and LPE had an effect in the total DMS accuracy score - one of the tests included in the studies - (chapter 2), while age and sex had a small effect in response time for correct items in one condition of the DMS (overt, i.e., target and responses are simultaneously on the screen). This supports the thesis that demographic variables affect cognition and need to be included in analyses and norm data.

A continuous norming or regression-based method norming method has been implemented since traditional methods to establish norms tend to be based on sub groups in the data, i.e., age brackets of two years (all $6-7$ years old children in one group), which leaves out the data of all other ages of the sample when establishing norms for each sub group (Van der Elst et al., 2011). For one, a large sample is needed to achieve statistically significant effects especially if sub groups are further divided based on other demographic variables that are included such as e.g., sex. Additional disadvantages concern the age sub groups boundaries (i.e., children with an age difference of one month are in different age sub groups) and specific sample characteristics of a sub group which may be atypical yet determine the norm for that subgroup. In contrast, the continuous norming method allows the inclusion of continuous (age) and dichotomous (sex and level of parental education) variables simultaneously (Van Breukelen \& Vlaeyen, 2005; Van der Elst et al., 2011) and was therefore used to establish norms on these EF tests in this sample of schoolchildren.

\section{Comparing Ukrainian norms to Western norms for collected EF tests}

In addition to the analyses of the influence of (combined) demographic variables in the Ukrainian sample on EF (and underlying cognitive function) test scores, a comparison to Western norms was carried out. The first focus in this study was to include demographic variables that may support understanding of cultural differences affecting 
performance in cognitive tests, but comparison of samples may also elucidate similarities or lack thereof. These analyses were mostly based on descriptive comparisons and used the traditional two-year age groups on which norms in the published literature for these tests are based. For the majority of executive - and underlying cognitive functions tests (i.e., for the DMS, PRM, SWM, and the SOC), the confidence intervals (CIs) overlapped between Ukrainian and Western populations - which indicates that the scores were comparable among these populations. In contrast, for one test included in the studies (chapters 2-4), the NNAT (i.e., paper-based matrices; chapter 4) mean score CIs of the Ukrainian sample did not overlap with the CIs of the Western mean scores, indicating a lower mean performance of children in the Ukrainian sample on this test compared to Western children. This appears to provide grounds that tests for which the scores were comparable between the two samples (the DMS, PRM, SWM, and the SOC but not the NNAT), norm scores may be applicable interchangeably between countries. However, more rigorous research is needed to prove equivalence among populations. Such research may e.g. consist of a comparison including other demographic variables (i.e., sex and LPE) and not only age (sub groups) as was done now (in chapters 2, 3, \& 4), for a more complete and thus valid comparison. Furthermore, the Ukrainian and Western samples were not compared for the Spatial Span (SSP) and Paired Associates Learning (PAL; chapter 3) due to differences in the analyzed outcome measures between the two samples. Yet, as concluded in chapters 2, 3 and 4, outcome measures of tests included correlated significantly, but were far from perfect, thus showing comparable as well as different underlying constructs. This underlines the inconclusive findings of the comparisons between the two samples on all tests used in the studies reported here, as well as the multi-dimensionality of executive- and underlying cognitive functions, and the need for additional research on norms for these functions including cultural comparisons.

\section{Developmental trajectories of EFs}

EFs appear not to develop at the same time and highly interconnected but unique EFs are thought to reach mature levels at different ages (Anderson, 2002; Luciana \& Nelson, 1998, 2002; Tau \& Peterson, 2010). (Curvi)linear development with age was noted, as expected (see above). However, stabilizing scores were found in younger and older children (a cubic effect in the relationship between age and scores, i.e., indicating less development at certain ages) on the PAL, SSP, SWM and SOC (chapters $3 \& 4$ ). Only further research may establish at which ages scores stabilized. Measurement (e.g., as presented in chapters 2-4) in turn may enable monitoring of EFs development. Contrary to earlier beliefs, interventions have been associated with improvement of scores on particular executive - (and underlying cognitive) functions including when trained from an early age (Diamond \& Lee, 2011). Rueda, Posner and Rothbart studied the emergence of selfregulation (2005) which is thought to be preceded by effortful control of among others attention, when resolving a conflict. They found improved attention (measured in shorter response times to resolve the conflict) after several weeks of attention training in a flanker 
task in $4-6$ years old. This is important because attention is thought to be needed for all other EFs, including the tests used in chapters 2 - 4 (Anderson, 2002). Importantly, EF skills were shown to be rather robust once they were established (Thomas \& Johnson, 2008).

Executive- and underlying cognitive functions have been associated with different basic constructs (Anderson, 2002; Hughes, 2011). The difference in structure of these cognitive functions also finds support in the different influences of demographic variables. The demographic variable LPE was found to have an effect (in addition to age) on visual recognition of and short-term memory for patterns (DMS and PRM), and problem solving as measured in the matrices of the NNAT test. EFs were shown to be sensitive to (disruptive) environmental influences, which is not surprising given the prolonged development of executive - and underlying functions from infancy into early adulthood (Hughes, 2011). Especially positively affecting LPE has been associated with advantageous outcomes in EFs interventions in early school years (Hughes, 2011). One intervention included parents directly. Hughes and Ensor (2009), e.g., found improvement in 2 - 4 years old children's EF scores after an intervention, involving parent's structured play with their child. However, not all EFs were found that easy to train, e.g., control of inhibition and/or more complex EFs such as problem solving (Hughes, 2011).

Both specific interventions and preventive programs show effects in $\mathrm{EF}$ development. Melby-Lervåg, Redick and Hulme (2016) reviewed WM training in e.g., computerized visual spatial WM programs. They found short-term transfer effects that translate to specific situations. In-depth analyses however, revealed that far transfer effects, that is applying the learned skill in for example other academic subjects, or after/over a longer period, were not found. Similarly, Resch, Rosema, Hurks, de Kloet, \& van Heugten (2018) found little effect in their review on EF interventions for children with acquired brain injury. However, identifying effective components revealed that metacognition (i.e., knowledge about one's learning) and/or the use of strategies was the primary effective component (which are/require EFs). Furthermore, computerized (drill based, i.e., repeated practice to achieve automaticity in line with Melby-Lervåg et al., 2016) exercises were linked to improvement on similar tasks. Multi-component interventions were proposed combining drill based practice, external aids and metacognitive strategy training (including far transfer e.g., what to do in real life situations based on the external reminder) in children with acquired brain injury (Resch et al., 2018). Furthermore, school curricula associated with improved EFs are social-emotional learning and mental health promotion programs, which showed improved problem-solving, decision-making as well as academic performance (Durlak et al., 2011; Weare \& Nind, 2011; Wells, Barlow, \& Stewart-Brown, 2003). These preventative measures show promise among others due to their integrated nature of learning EFs. However, among thousands of programs only 17 controlled trials were found, signaling the need for more rigorous research (Weare \& Nind, 2011). Measuring EFs as reported in this thesis (chapters 2-4) may provide the starting point for monitoring EF development and considering interventions. 
Concluding this first part of the dissertation confirms the importance of research into EFs in not only Western but also other populations (e.g., Ukrainian children). We found support for the hypothesis that executive - and underlying cognitive functions are influenced differently by the demographic variables age, sex and LPE. Norms were calculated for the reported tests for children in Southwestern Ukraine, including these demographic variables. Finally, the road from measurement to monitoring progress in development of EFs was considered through potential interventions.

Aim 2: The second objective of this study was to explore variables related to the well-being of Ukrainian primary- and middle school children and their environment, i.e., the children's families, communities and society.

Development, according to the biosocial-psychological model (Bronfenbrenner \& Morris, 2006) is influenced by environmental factors from the child's immediate surroundings (home) to an increasingly wider range of reciprocal relations that tend to increase with age (i.e., school, community, society). An environmental variable that teachers expressed concern about in informal discussions in school in general about learning and teaching, was the availability of alcohol in communities. The perceptions of health, alcohol misuse and perceptions about people who misuse alcohol in (south) West Ukraine have therefore been explored and were reported in the second half of this dissertation (chapters 5 and 6). Ukraine is among a group of former Soviet countries that have been linked to significant levels of alcohol use (WHO, 2018). Research has shown that alcohol use has been associated with disadvantageous life outcomes, such as misuse that affects the health of individuals, risk taking behavior, parenting styles, early onset alcohol use and risk of alcohol dependence, effects on cognitive development, and a range of diseases and mortality (Burlaka et al., 2017; Jacob, Moser, Windle, Loeber, \& Stouthamer-Loeber, 2000; Polshkova et al., 2016; Popova et al., 2019). Furthermore, alcohol misuse has been associated with anxiety, depression, and alcohol use disorder, and has been reported to be a severely stigmatizing condition both for the person affected as well as their environment (e.g., family; Corrigan \& Miller, 2004; Schomerus et al., 2010).

In chapter 5 the focus was on inventorying the perceptions of alcohol use, perceptions about its relation to health, and whether these perceptions differ between different stakeholders such as youths and adults in Ukraine. Overall, the results indicated an omnipresence of alcohol from an early age. Descriptive and injunctive norms played a role in relation to daily alcohol use. Accessibility and low pricing (also related to unrecorded alcohol) resulted in alcohol being available from early childhood. Perceptions of normality of alcohol consumption stemmed from daily family use, cultural celebrations, and beer being perceived as water. However, wine and beer were perceived as drinks for 'weak' people while consumption of strong spirits was perceived as 'cool' and for 'strong' people. Onset of alcohol use outside the house was described from early adolescence (in line with Currie et al., 2004; reportedly 10-13 years old). People recovering from alcohol dependence ( $\mathrm{AD}$, defined in methods chapters 5 \& 6 ) stated in line with multiple stakeholder reports, 
that they had already formed a habit of regular alcohol use between 12 - 14 years old, and some may even have started below 10 years of age. Alcohol consumption, after the initial effect of happiness wore off, was associated with negative affect and used as a coping strategy. However, any discussion of feelings, especially in men, was not expected and discouraged. Respondents further mentioned a variety of negative consequences of alcohol misuse: Risk of unhealthy drinking patterns (i.e., binge drinking), underage alcohol use, AD and effects on health of the person affected and their family (violence, (attempted) suicide) were reported.

Furthermore, in chapter 6, public stigma was reported in people suffering from $\mathrm{AD}$ and their families, which may increase the risk of $\mathrm{AD}$, other forms of stigma and additional mental health problems in persons subjected to stigmatizing beliefs. These public harmful beliefs in society, based on external characteristics of an individual in this sample were thought to give rise to several forms of stigma: Stigma by association (attributing mentioned public perceptions to individuals close to the person who is subject of stigma, e.g., their children), self-stigma (a person believes and internalizes the harmful public perceptions, e.g., the experience of shame and guilt), structural stigma (structures in society enable stigma happening, e.g., refusing jobs to individuals suffering from AD), and provider-stigma (people in official roles or organizations stigmatize, e.g., people are refused treatment if they are alcohol dependent) - all of which were reported by respondents, in teaching and in health care. Multidimensional strategies to reduce stigma were discussed as both structures in society that 'cause' stigmatizing perceptions need to be addressed as well as the needs of people who are stigmatized and possibly of those who stigmatize. Structures in society in the rural part of the reported sample (chapters 5-6) concern access to clean water (and thus alcohol was perceived as the 'clean' drink in general). Respondents from multiple backgrounds reported on insufficient access to work and limitations in policy on alcohol (which the WHO also found to be absent in 2014) e.g., on pricing, unrecorded alcohol and underage access, and mental health services.

Importantly, as reported in the sample (from 14 years and older) and in line with the literature the quantity and patterns of alcohol use have shown a significant increase since the early 1950s in the former USSR, across changing political systems; and repeated attempts to regulate alcohol consumption occurred as the economic and social impact was noticed (Currie, 2004; Landberg, 2008; Reitan, 2001). This supports the notion that despite the valuable lessons from earlier implementation attempts of regulation, the values, beliefs, and norms of a population need to be included. Here the reported norms combined with negative affect (feelings, depression, anxiety) and prohibition to discuss these (potentially contributing to limitations in coping strategies for life events) need to become subject of discussion in multiple layers of society, including government. International (successful) examples may accelerate this process and help inform policy (Marmet, Rehm, Gmel, Frick, \& Gmel, 2014; Pedersen et al., 2013; Peeters et al., 2015); Neuner-Jehle, Schmid, \& Grüninger, 2013). 


\section{Methodological considerations}

The first aim of the current dissertation concerned the measurement of executive(and underlying cognitive) functions and norming of these EFs in a novel sample, i.e., Ukrainian children (5.5 to 14.5 years old). The CANTAB ${ }^{\circledR}$ EF tasks have been validated for use in children (Luciana \& Nelson, 1998, 2002), however computerized EF tasks such as employed in the current research may lack ecological validity, particularly from the perspective of Ukrainian students (Hughes, 2011). Measuring response times implies sitting in a separate room without distractions, which does for instance not reflect natural classroom practice that inherently has distracting factors. Tools that include other dimensions of EF measurement such as a standardized practical task (e.g., following recipe instructions) showed good discriminant validity (i.e., ability to fulfill task demands), concurrent validity (i.e., between the practical task and traditional EF test measures) and interrater test-re-test reliability (Chevignard, Catroppa, Galvin, \& Anderson, 2010). Such practical tests may more resemble classroom and/or real life situations as other environmental factors and/or people require a person to complete a task while using varying EFs e.g., ignoring likely multiple distractors and incorporating multiple sources of knowledge and skills into one's plan. Accompanying the measures reported here with more 'ecological' tasks may especially elucidate understanding of EFs levels in daily life (Hughes, 2011). An ecological approach to testing may also alleviate concerns related to the cultural appropriateness of non-verbal tests. Non-verbal tests in general and visual spatial tests specifically, such as copying figures have been found to be embedded in the culture of the test designer, which may differ from the participant doing the task (Roseli \& Ardila, 2003) and as such bias the results of such tests. The CANTAB ${ }^{\circledR}$ tests used in this study were designed in and for Western societies and thus subject to Western bias in test design but also in norms among others e.g., the effect of level of parental education on child scores (Cambridge Cognition, 2012; Robbins et al., 1998). Apart from norming different samples as proposed in this study, additional group comparisons (different age groups within a culture and the same age groups between cultures) may elucidate cultural test characteristics within the test tool itself. Such differences could contribute to understanding the ecological validity of tests in different cultures in more detail compared to the current findings of demographic effects in this study (Ardila, 2006),

Furthermore, this was a cross-sectional study, which may provide a valuable starting point for establishing demographically corrected norms in different samples (chapters 2, 3, 4), but prohibit any causal interpretations due to sampling bias (i.e., nonrespondents may have had and/or shared particular characteristics which were not analyzed) (Sedgewick, 2014). Furthermore, the cross sectional data were a single measurement point in time, which is insufficient to make an inference between risk factors and outcomes (Sedgewick, 2014). Longitudinal studies (following a cohort from e.g., 4 - 21 years old) may provide additional support for developmental trajectories of EFs in schoolchildren in this sample and inform patterns of (a)typical development (Thomas et al., 2009). 
Qualitative research carries the strength of the possibility to report complex and detailed data related to the participants and the topic of research in depth (Anderson, 2010). However, in general researcher bias needs to be considered in qualitative data. The researcher's presence for example during interviews may affect participant's responses (Anderson, 2010). In this thesis (chapters $5 \& 6$ ), risk factors (the availability of alcohol) in development were related to the concern of teachers. However, there appear more and possibly also significant risk factors, such as the impact of the Chernobyl disaster, absolute poverty and nutrition deficiency, limitations in access to further education, and multiple sources of stress. These have not been researched in this dissertation and may each or in combination play a significant or possibly causal role. Hence, the recommendation is made to look at protective strategies for child development. Furthermore, the qualitative data provided a rich account of individual's lived experiences, but have limited generalizability for the whole of Ukraine. Large scale research is especially needed to confirm the perceptions of public stigma and other forms derived from it, as well as to inform interventions needed for multiple stakeholder groups (chapter 6). Especially stigma related to female alcohol use and pregnancy requires further research as they may impact health behaviors in pregnancy. Importantly, some incidental remarks could not be reported (single mention), yet the role of females appears more complex than expected given Ukraine's multi-ethnic background (e.g., the strong female in leadership roles, versus the female who is to serve family needs). Established tools or validated adaptations are needed to measure stigma in those subjected to stigma, and those stigmatizing, in order to understand the components and possibly the different weights of the components in the context of this population (Link, Yang, Phelan, \& Collins, 2004).

\section{Conclusions and recommendations}

To conclude, the studies reported in this dissertation provide valuable starting points for further research and intervention development among vulnerable youth in Ukraine. Relatively novel regression-based analyses allowed to establish demographically corrected norms for this sample. Varying influences of the demographic variables age, sex and LPE on the children's performances on cognitive tasks were found. EF measurement and interpretation of scores using suitable norms are important to ascertain whether performance is within the normal range. Establishing the normative range may also help to monitor developmental patterns i.e., a child's score may be below the norm consistently but the trajectory may resemble the population's trajectory. Thus, an established normative range for a population supports interpretation of scores and may inform interventions. EFs can, albeit not easily be trained from a young age. The established demographic influences need to be incorporated in interventions. This is relevant due to the role of EFs in academic, social and emotional performance. Health behaviors tend to be grounded in norms, beliefs and personal characteristics (EFs), and are hard to change. Different forms of stigma and 


\section{Chapter 7}

stigmatizing beliefs in society were associated with alcohol misuse. Problems related to alcohol and associated stigmatizing beliefs may affect children, their parents, and/or their families. Integrated social-emotional training to enhance protective factors for advantageous life outcomes, i.e., self-efficacy, specific EF training, metacognitive strategylearning and social-emotional learning was therefore advocated. Key competencies in social-emotional learning in the skill of self-awareness include a sense of self-efficacy and self-confidence, while the skills of self-management include goal setting. To support students with coping skills e.g., to resist peer pressure, or an elder offering an alcoholic drink to an underage adolescent an integration of social-emotional learning in the traditionally 'cognitive domain' of education is advocated and implementation may provide opportunities for pro-social norm development and anti-stigma attitudes in among others educators (Jones \& Bouffard, 2012). This is important because increased EFs development has been linked to advantageous outcomes in academic learning (Hughes, 2011) and life (Eisenberg et al., 2019). 


\section{Summary}

Advantageous learning outcomes have been linked to beneficial life outcomes such as employability (Parsons \& Bynner, 1997). Executive functions (EFs) in turn have been associated with successful learning outcomes (Hughes, 2011). The first objective of this dissertation (chapters 2 - 4) was to collect data to establish norms for tests measuring executive functions and underlying cognitive functions in a sample of schoolchildren in Southwest Ukraine. The second aim of this thesis (chapters 5 \& 6) was to investigate perceptions of Ukrainian adolescents and their families, communities and society on health and more precisely on alcohol (mis)use in West Ukraine.

For the first aim of this thesis $\mathrm{CANTAB}^{\circledR} \mathrm{EF}$ tests were used. Demographic variables have been associated with cognition and an increased relevance of the interpretation of test scores in clinical and research studies. Demographic variables showed an effect on EF test scores but were different per outcome measure (chapters $2-4$ ).

In chapters $2-4$ data were collected to evaluate the influence of the demographic variables age, sex, and level of parental education (LPE) on executive function tasks in schoolchildren 5.5 - 14.5 years old in Southwest Ukraine. Demographic variables have been associated with executive - (and underlying cognitive) functions test performance and therefore test scores in relation to characteristics of this non-Western sample were examined (Lezak et al., 2012). Multiple regressions resulted in continuous norms for these EFs tests.

Chapter 2 concerned data collection and norming for the computerized CANTAB ${ }^{\circledR}$ Delayed Matching to Sample (DMS; Cambridge Cognition, 2012) task, which is thought to test visual matching and short-term recognition memory in non-verbalisable patterns. The total score, overt (simultaneous presentation of target and response items) and covert (delayed presentation of response items after the target is covered) conditions respectively are presented. Accuracy scores and the probability of an error after an incorrect response score developed with age and higher level of parental education in the expected direction. However, only the demographic variable sex was associated with response latencies; that is boys worked faster without making more mistakes compared to girls. Norms were calculated for these measures.

The separate analyses of the overt and covert conditions showed an age effect in accuracy in both conditions and for response time only in the overt condition. LPE was implicated in the DMS accuracy, but only in the overt condition. Sex differences were only found in response times in the overt and covert conditions; boys had shorter response times in both conditions.

In chapter 3, visual, spatial, and/or visual spatial short-term memory (STM) was thought to be evaluated using three computerized tasks: the Pattern Recognition Memory (PRM; visual STM) task, the Spatial Span (SSP; spatial STM) and the Paired Associates Learning (PAL; visual spatial STM) task, respectively (Cambridge Cognition, 2012). Moderate Pearson correlations revealed that these tasks measured related yet unique 
constructs. The PRM scores increased curvilinear with age; scores increased more with age in younger children compared to older children. In contrast, PAL and SSP scores showed cubic age effects, that is scores were relatively stable (i.e., showing less increases at certain ages) at several points in the cross-sectional trajectory (as opposed to (curve)linear effects), i.e., in the youngest and oldest children. Additionally, visual and visual spatial STM scores (PRM and PAL) improved as a function of higher levels of parental education (LPE). LPE however was not a significant predictor for the SSP score (spatial STM). The visual STM task was thought to be processed in the ventral pathway (colors and features of a shape) and the spatial components in the SSP were related to the dorsal pathway (location and motion), while the visual spatial task (PAL) was thought to place demands on both.

In chapter 4 more complex EFs involved in (planning and problem solving) goal setting were evaluated; the Spatial Working Memory (SWM; thought to be mainly planning) and the Stockings of Cambridge test (SOC; thought to be mainly problem solving), and the Naglieri Nonverbal Ability test (NNAT; matrix problems) (Cambridge Cognition, 2012; Nagliery, 2003). Correlations indicated the measurement of related yet unique underlying constructs in planning (as part of cognitive flexibility) and goalsetting (including reasoning). Evaluating the influence of demographic variables showed an increase in accuracy with age on these three tasks. The NNAT scores showed a curvilinear increase with age and higher scores were associated with higher levels of parental education. The SWM and SOC showed a cubic relation with age, that is stabilizing scores at younger and older ages. The demographic variable sex was not significant.

Last, the scores of this Ukrainian sample were compared to the (Western) test norm scores in chapters 2, 3 and 4 respectively. The DMS, PRM, SWM and SOC showed comparable score ranges for both geographical regions, except for the lower mean in $6-7$ years old children in the sample in the PRM. This might relate to differences in early years school systems. However, the scores for planning, (cognitive flexibility) and goal setting (reasoning) task NNAT did not overlap between the Western norm groups and the Ukrainian sample, while EFs were thought to develop well into young adulthood.

The second part of this dissertation (chapters 5 and 6 ) consisted of qualitative research into teachers' concerns about alcohol use in communities. The WHO (2018) prioritizes decreasing unhealthy alcohol use to reduce the global burden of non-infectious disease. Ukraine falls in the highest WHO category of 'years of life lost' due to alcohol use (WHO, 2018). The purpose of the study described in chapters 5 and 6 was to investigate current perceptions of alcohol consumption in relation to health. In-depth semi-structured interviews with various adult stakeholders and open-ended written questions administered with younger participants (12-21 years old) were analyzed.

Norms emerging from the data (chapter 5) indicated a 'Mediterranean' pattern of daily alcohol use (i.e., wine with meals and beer to still thirst). Perceptions and habits were explained in terms of historical, economic and cultural contexts. However, childhood sipping, the reported quantity and patterns of binge-drinking indicated a risk to health. Additionally, access to (strong and/or unrecorded) alcohol for all ages was reported (i.e., availability and low pricing). Furthermore, beer was perceived as the beverage for 'weak' 
people while consumption of spirits was described as being 'strong' and 'cool'.

Adolescents described peer pressure linked to alcohol consumption. Surprisingly, people showing signs of alcohol dependence (AD) were equally labelled as 'weak'.

Simultaneously, part of youth expressed disadvantageous perceptions of children/peers who (had) started to consume alcohol from an early age (and their parents).

A culture for men not expected to talk about feelings was reported while alcohol was described as a coping mechanism. Alcohol use was reported as initially providing positive affect, but followed by negative affect (depression, anxiety) and thought to indicate low-self-esteem. AD of parents was reported in relation to disadvantageous parenting styles. AD survivors and multiple other sources reported establishing a pattern of continued alcohol consumption outside the home for some between $12-14$ years old. Part of youths, in contrast reported long-term goals (e.g., higher education, physical and mental health) and reported to pursue abstinence from alcohol.

Different forms of stigma reported in chapter 6 emerged from the data. Public stigma, that is labelling people experiencing alcohol dependence (AD) as weak or lacking in self-control, is a structural form of stigma from which other forms are derived. Stigma by association was reported in relation to children and partners of people with AD. Peers, other adults and teachers were reported as sources for stigmatization. Self-stigma was reported, i.e., a child of a person with AD may believe and internalize the harmful public perceptions. Structural stigma appeared e.g., in a lack of rehabilitation facilities. Provider-stigma was reported in people in official organizations/roles in society e.g., hospitals. Stigma appeared implicated in female alcohol use and especially in relation to pregnancy. Data suggested that females tried to hide alcohol use. Patterns emerging from these data need further research as stigma has been proven to prevent individuals from accessing support and thus potentially continuing risky behavioral patterns. Given the proven detrimental effects of stigma to the (mental) health of people being stigmatized, research of the stigmatized groups, quantifying the perceptions and mechanisms of stigmatization, and policy and interventions at all levels are needed. Chapter 7 consists of a summary of the main findings of the five studies (chapters 2 - 5) presented in this thesis, followed by a discussion and reflection on limitations of the studies as well as recommendations for further research. 


\section{Samenvatting}

Positieve leerresultaten zijn gekoppeld aan bevorderlijke levensomstandigheden zoals bijvoorbeeld het krijgen en behouden van werk (Parsons \& Bynner, 1997). Executieve functies (EFs) worden op hun beurt weer in verband gebracht met positieve leerresultaten (Hughes, 2011). Het eerste doel van deze dissertatie (hoofdstuk 2 - 4) was het verzamelen van data om normen te berekenen voor executieve (en onderliggende cognitieve) functie testen in een steekproef van schoolkinderen in Zuidwest Oekraïne. Het tweede doel van de dissertatie (hoofdstukken 5 \& 6) was de percepties te onderzoeken van Oekraïense adolescenten, hun families en directe omgeving omtrent gezondheid en meer precies alcohol (mis/) gebruik in West Oekraïne.

Voor het eerste doel zijn CANTAB ${ }^{\circledR}$ testen gebruikt. Demografische variabelen beïnvloeden cognitie en zijn daarom in de analyses meegenomen. De relevantie van de interpretatie van testscores in klinische- en onderzoek studies neemt toe als normen demografisch gecorrigeerd zijn. Demografische variabelen hadden invloed op de EFs tests, maar de effecten verschilden per uitkomstmaat.

In de hoofdstukken 2 - 4 werden gegevens verzameld om de invloed van de demografische variabelen leeftijd, sekse, en niveau van opleiding van de ouders, op executieve functie test scores vast te stellen. Normen voor deze testscores werden berekend van schoolkinderen van 5.5 - 14.5 jaar oud in het zuidwesten van de Oekraïne.

Demografische variabelen zijn geassocieerd met executieve (en onderliggende cognitieve) functies en dientengevolge met test resultaten. Testscores in relatie tot karakteristieken van deze niet westerse steekproef zijn onderzocht (Lezak et al., 2012). Multiple regressies resulteerden in continue normen voor deze EFs tests.

Hoofdstuk 2 ging over het verzamelen en normeren van gegevens voor de gecomputeriseerde CANTAB ${ }^{\circledR}$ Delayed Matching to Sample-taak (DMS; Cambridge Cognition, 2012), waarvan wordt aangenomen dat deze het visuele spatiale korte-termijngeheugen (KTG) van gekleurde abstracte patronen test. Open en afgedekt (vertraagde) item-opties werden gepresenteerd. De hoeveelheid correct nam toe met leeftijd en hoger niveau van de opleiding van de ouders. Alleen de demografische variabele sekse was geassocieerd met reactietijden; dat wil zeggen jongens werkten sneller zonder meer fouten te maken in vergelijking met meisjes.

De toegevoegde analyse van de open en afgedekte conditie liet een leeftijdseffect zien in beide condities en in response time alleen voor de open conditie. Het opleidingsniveau van ouders had alleen een effect op de hoeveelheid correct in de open conditie. Sekse verschillen werden alleen gevonden in de reactietijden in de open en afgedekte conditie; jongens reageerden sneller dan meisjes in beide condities.

In hoofdstuk 3 werden het visuele, ruimtelijke en het visueel ruimtelijke KTG verder geëvalueerd door middel van drie gecomputeriseerde taken: de Pattern Recognition Memory (PRM; visueel KTG), de Spatial Span (SSP; ruimtelijk KTG) en Paired Associates Learning (PAL; visueel ruimtelijk KTG) taak, respectievelijk (Cambridge Cognition, 
2012). Matige Pearson-correlaties onthulden dat deze taken gerelateerde maar ook unieke componenten meten. De PRM-scores namen met leeftijd curve-lineair toe; de scores namen meer toe met leeftijd bij jongere kinderen in vergelijking met oudere kinderen. De PAL- en SSP-scores vertoonden daarentegen kubische leeftijdseffecten, dat wil zeggen leeftijdgerelateerde stijgingen in scores waren relatief stabiel op verschillende punten in deze cross-sectionele (grafiek)lijnen, d.i., bij de jongste en oudste kinderen (in tegenstelling tot (curve)lineaire effecten). Daarnaast verbeterden visuele en visuele ruimtelijke KTG-scores (PRM en PAL) als een functie van hoger opleidingsniveau van de ouders, maar niet voor ruimtelijke KTG (de SSP taak). De visuele componenten in KTG-taken worden geassocieerd met de ventrale route (kleuren en kenmerken van een object). De ruimtelijke componenten zoals in de SSP waren gerelateerd aan de dorsale route (locatie en beweging), terwijl de visuele ruimtelijke taak (PAL) werd geassocieerd met beide routes in de hersenen.

In hoofdstuk 4 werd de invloed van dezelfde demografische variabelen op de EFs van planning en probleem oplossen en het stellen van doelen geëvalueerd. De taken Spatial Working Memory (SWM; vooral geassocieerd met planning), de Stockings of Cambridgetest (SOC; vooral geassocieerd met probleem oplossen) en de Naglieri Nonverbal Abilitytest (NNAT; matrix problemen) worden verondersteld deze EFs te meten (Cambridge Cognition, 2012; Nagliery, 2003). Correlaties duiden op de meting van gerelateerde maar unieke onderliggende componenten in planning (als onderdeel van de EF cognitieve flexibiliteit) en doelen stellen (inclusief redeneren). De NNAT-scores vertoonden een curvilineaire toename met de leeftijd en hogere scores waren ook geassocieerd met een hoger opleidingsniveau van de ouders. De SWM en SOC vertoonden een kubieke relatie met leeftijd, dat wil zeggen dat scores stabiliseerden op jongere en oudere leeftijd. De demografische variabele sekse was niet significant.

Als laatste werden de scores van deze Oekraïense steekproef vergeleken met de (westerse) testnormscores in de hoofdstukken 2, 3 en 4 respectievelijk. De DMS, PRM, SWM en SOC vertoonden overlappende score intervallen voor deze twee verschillende proefpopulaties, behalve de lagere gemiddelde score van kinderen van 6 - 7 jaar oud in de PRM. Dit zou gerelateerd kunnen zijn aan verschillen in het schoolsysteem in de eerste leerjaren. Echter, de scores voor planning (cognitieve flexibiliteit) en doelen stellen (inclusief redeneren) zoals getest in de NNAT vertoonden geen overlap tussen de westerse normgroepen en de Oekraïense steekproef. Deze EF's worden verondersteld zich tot ver in de jongvolwassenheid te ontwikkelen.

Het tweede deel van dit proefschrift (hoofdstuk 5 en 6 ) bestond uit kwalitatief onderzoek naar aanleiding van de zorg van leraren omtrent alcohol gebruik in lokale gemeenschappen. De WHO (2018) geeft prioriteit aan het reduceren van ongezond alcoholgebruik om wereldwijd de lasten van niet-overdraagbare ziekten terug te dringen. Oekraïne valt wereldwijd in de hoogste WHO-categorie van 'verloren levensjaren' door alcohol gebruik (WHO, 2018). Het doel was om de huidige percepties van alcoholgebruik in relatie tot gezondheid te onderzoeken. Er werden semigestructureerd interviews met 
verschillende volwassen partijen, en schriftelijke open-vragen lijsten met jongere deelnemers (12-21 jaar oud) gedaan.

Normen die uit de gegevens naar voren kwamen wezen op een 'Mediterraan' patroon van dagelijkse alcoholconsumptie (wijn met maaltijden en bier om dorst te lessen). Percepties en gebruiken werden beschreven in historische, economische en culturele contexten. Kinderen die alcohol 'proeven', de gerapporteerde hoeveelheid en patronen van alcohol gebruik vormen echter een gezondheidsrisico. Daarnaast werd toegang tot (sterke en/of niet-geregistreerde) alcohol voor alle leeftijden gerapporteerd (d.w.z. beschikbaarheid en lage prijzen). Bovendien werd bier gezien als de drank voor 'zwakke' mensen, terwijl het gebruik van sterke dranken werd omschreven als 'sterk' en 'cool'. Adolescenten beschreven groepsdruk van leeftijdsgenoten gerelateerd aan alcoholgebruik. Verrassend genoeg werden mensen die tekenen van alcoholafhankelijkheid vertoonden ook als 'zwak' bestempeld. Tegelijkertijd toonde een deel van de jongeren een ongunstige perceptie van kinderen/leeftijdsgenoten die al op vroege leeftijd alcohol begonnen te consumeren wat deels geassocieerd was met de ouders van die kinderen.

Een cultuur waarin het van mannen niet verwacht wordt om over gevoelens te praten, werd gemeld terwijl alcohol werd beschreven als een coping-mechanisme. Alcohol werd omschreven als aanvankelijk een positief effect te hebben, maar dat werd gevolgd door negatief affect (depressie, angstgevoelens) en gedachten die wijzen op een laag gevoel van eigenwaarde. Afhankelijkheid van alcohol van ouders werd gerapporteerd met betrekking tot nadelige opvoedingsstijlen. Meerdere bronnen, onder andere na rehabilitatie, rapporteerden een patroon van aanhoudende alcoholconsumptie buitenshuis die voor sommigen tussen 12 tot 14 jaar oud was begonnen. Een deel van de jongeren rapporteerde daarentegen lange-termijn doelen (bijv. hoger onderwijs, fysieke en mentale gezondheid) en meldde onthouding van alcohol gebruik na te streven.

Verschillende vormen van stigmatisering gerapporteerd in hoofdstuk 6 kwamen uit de data naar voren. Publiek stigma, dat is het labelen van mensen die van alcohol afhankelijk zijn als 'zwak' of met een 'gebrek aan zelfbeheersing'. Stigma door associatie werd gerapporteerd met betrekking tot kinderen en partners van mensen met afhankelijkheid van alcohol. Leeftijdsgenoten (adolescenten), andere volwassenen en leraren werden gerapporteerd als bronnen van stigmatisering. Zelfstigma werd gemeld, d.w.z. een kind van een persoon met afhankelijkheid van alcohol gelooft en internaliseert de schadelijke publieke percepties. Structureel stigma bleek uit de rapporten door een gebrek aan rehabilitatievoorzieningen. Stigma in voorzieningen werd gemeld bij mensen in officiële organisaties/rollen in de samenleving, bijvoorbeeld in ziekenhuizen. Stigma leek gerelateerd aan het gebruik van alcohol door vrouwen en vooral tijdens de zwangerschap. Vrouwelijk alcoholgebruik leek verborgen plaats te vinden. Patronen die uit deze gegevens naar voren komen, moeten nader worden onderzocht, aangezien is aangetoond dat stigma individuen ervan weerhoudt om toegang te zoeken tot hulpverlening en dus potentieel risicovolle gedragspatronen worden voortgezet. Gezien de bewezen schadelijke effecten van stigma op de (mentale) gezondheid van mensen die worden gestigmatiseerd, is onderzoek naar de gestigmatiseerde groepen nodig, zoals het kwantificeren van de 
percepties en mechanismen van stigmatisering, beleid en interventies op verschillende niveaus in de samenleving. In hoofdstuk 7 werd een samenvatting van de hoofdbevindingen van de vijf studies (hoofdstuk $2-5$ ) in de thesis gepresenteerd gevolgd door een discussie, een reflectie op beperkingen van de studies en aanbevelingen voor verder onderzoek. 


\section{Valorization}

The first aim of this dissertation was to contribute to scientific knowledge concerning norms for several tests measuring cognition and more specifically executive functions in a non-Western (Ukrainian) sample of schoolchildren. The second aim was to investigate perceptions of Ukrainian adolescents and their families, community and society about health in general and more specifically alcohol (mis)use. The significance of the findings for future studies and interventions has been described in the previous chapters. This valorization section aims to describe the social and practical value and relevance of the findings in this dissertation project for individuals, communities, and/or organizations (e.g., children, parents, schools, school psychologists) outside the scientific community.

\section{Relevance}

Executive functions (EFs) is an umbrella term used for a set of interdependent cognitive skills that support decision-making, problem solving, and planning, which all require cognitive flexibility (Anderson, 2002). These skills have been shown to support beneficial outcomes in daily activities, e.g., obtaining and keeping jobs (Duncan et al., 2007; Parsons \& Bynner, 1997). The development of EFs takes up until (at least) young adulthood and the level of developmental end state of functioning differs per person (Luciana et al., 2005). Indeed, EFs are rather sensitive to disadvantageous developmental factors such as, among others, lower socio-economic status and stressful life events (Bos et al., 2009). Thus it is important to monitor development of EFs in schoolchildren, particularly those considered to be 'at risk', and to identify challenges (Luciana et al., 2005; Luciana \& Nelson, 1998).

We therefore measured multiple EFs in Ukrainian schoolchildren (5.10 to 14.5 years old, reported in the chapters $2-4$ ) and established a suitable and culturally appropriate norm. Cognitive functions and specifically EFs have been associated with knowledge, values and beliefs, which differ per culture and social context (Ardila, 2005; Lezak, Howieson, Bigler, \& Tranel, 2012). Ukraine, with its complex history (e.g., geopolitical changes affecting among others economic factors such as work opportunities) may share cultural characteristics with other Slavic and/or former USSR countries (D'anieri, 2018; Berlach, Kulyk, \& Losych, 2019; Lekhan, Rudiy, \& Richardson, 2010; Kubicek, 2002; Subtelny, 2009). The norms established for these EFs tests in this study, included several demographic variables to reflect the sample's characteristics, and are therefore appropriate for these Ukrainian schoolchildren as opposed to the existing Western norm (De Luca et al., 2003; Lehto, Juujärvi, Kooistra, \& Pulkkinen, 2003; Luciana \& Nelson, 2002). This information is relevant for varying communities to provide the normal range for developmental areas (e.g., EFs) or measurement tools (e.g., tests and their accompanying norms), that are currently still mainly reported based on Western data. Having appropriate norms against which to compare children's performance may enable 
early intervention if (extreme) deviations from these norms are found. Furthermore, the method of norming described in chapters $2-4$, supports the replication and application of these tests in other samples, as well as for different tests or measurement tools with the aim to increase suitable evaluation of children's scores.

The relevance of the second aim of this dissertation relates to a holistic developmental view on early childhood (Bronfenbrenner, 1994). Bronfenbrenner and Morris (2006) posed that child development needs to be seen in its context; well-being is not only related to individual characteristics but also to the environment (e.g., interpersonal variables, society and culture). Translating this general holistic view to the Ukrainian sample: Teachers had expressed concerns about the availability of alcohol to young people and their potential alcohol consumption, as well as possible alcohol (mis)use in families. Therefore, we focused on children's developmental context by investigating the perceptions about health (and alcohol) from different stakeholder perspectives and the viewpoints of varying age groups in the second part of this dissertation (chapters $5-6$ ). This is relevant as it provides qualitative support for figures presented by e.g., the WHO (2018). Risky alcohol use worldwide has been identified as a priority and Ukraine scores in the highest category of the so-called 'years of life lost' (WHO, 2018). Indeed, our findings reflected the country's complex history, economic challenges as well as cultural traditions of a Mediterranean pattern of alcohol consumption with family meals and norms related to hospitality (in line with e.g., Popova et al., 2007). Furthermore alcohol use was discussed by both adults and youth in the context of social norms and coping mechanisms. The qualitative data presented here, therefore point toward and refine specific needs of different groups within Ukrainian culture, and they inform the feasibility of policies aimed at e.g., reducing the production and sales of home-brewed alcohol.

The findings reported in chapter 6 are relevant in relation to the prevention of public stigma, which was reported to exist in people suffering from AD and their families (Bos et al., 2013). Knowledge on stigma and the particular forms of stigma is relevant since a wide range of structural measures and interventions are needed to address these harmful beliefs in society and organizations (Bos et al., 2013; Hatzenbuehler et al., 2013). Further, fear of being stigmatized has been identified as an important barrier to seeking help and therefore interventions need to focus on reducing this barrier at the individual level and increasing feelings of self-efficacy and coping strategies (Bartholomew-Eldridge et al., 2016; Schomerus et al., 2010).

\section{Target groups}

The knowledge and data generated with the studies in this dissertation may be of interest to a wide-ranging audience.

Educational professionals (psychologists, social workers) working with children adolescents may gain better insight in potential levels and trajectories of EFs development by administering the EF tests described in this thesis. Establishing Ukrainian norms for valid EFs tests as well as a comparison to Western norms allows application in real-life 
settings for the sample. For example, these norms can be used for appraisal of individual performance and may help to inform long-term goals such as starting to monitor EF development. These norm calculations furthermore provide a starting point for more objective evaluations of classes, year groups, schools and individual children, compared to e.g., teacher observations. Professional decision-making includes referral to secondary schools and further education. Objectively obtained reports on skills (e.g., EFs) may provide more reliable tools to inform choices about suitable further academic development.

Organizations publishing (established) norms and populations that differ from generally established test norms may gain from the refined knowledge on creating norm data in schoolchildren as we presented in this dissertation. The regression-based methods described extensively in Chapters $2-4$ are a rigorous and evidence based approach to norming, resulting in more appropriate standards for populations other than possibly established for current tests. This norming procedure might be applied to varying populations that 'differ' from existing norms. Existing test norms may incorporate a limited amount of demographic variables, and thus be limited in the standard against which an EF test score is compared. This means that both characteristics of the norms (e.g., only including one demographic variable such as age) as well as (sub)populations may be in need of more suitable norms. To say it differently, a well-established Western norm for valid measurement tools may be limited in its suitability due to the limitations in the method of calculating norms. As such, this study may be of relevance to organizations providing normed tests. Apart from these established norm samples, populations that have not been included in norm calculations for valid EF tests, such as the non-Western schoolchildren in this dissertation, may also benefit from applying the methods described in detail.

Parents and caregivers may gain insight in their child's development through scores on EFs, which are compared against an appropriate norm for their child. Parents, especially in stressful life situations (e.g., lower socio-economic status, or suffering from alcohol misuse in the family) may benefit from seeing results of validated EFs measurement compared against suitable norms. A developmental message about a general cognitive skill of their child (e.g., the EF of short-term/working memory) may provide the chance for a constructive discussion about their child's needs and well-being as opposed to an attainment score on a general knowledge test. As such parents may be supported in their self-determination to raise their children (e.g., knowing and understanding when to ask for help).

Target groups for the second aim of this dissertation overlap with the ones mentioned above, yet also include wider parts of communities and society. For example, stigma concerns society and implicates measures at the societal level. Yet, the current research also indicated that stigmatizing perceptions of individuals exist at lower, organizational levels. Provider stigma was reported in relation to health care, but also female versus male patients was. Thus the availability of care (e.g., health insurance accessible for all) as well as underlying beliefs of some professionals could be targets of intervention (Angermeyer et al., 2013). Well-meaning respect for privacy may suggest a 
barrier to health care, which needs further research. Equally, this example demonstrates that varying forms of stigma need to be understood thoroughly within the applicable groups, including the perceptions, attitudes and underlying beliefs (i.e., the mechanisms). Although clear areas in need of intervention emerged, more specific needs assessments are required first.

Another area where organizational level interventions may merge with interpersonal and personal levels could concern schools. Worldwide, a range of issues in primary and middle schools (such as risk for the initiation or continuation of substance use, academic failure, peer pressure, and mental health problems (Eaton et al., 2008) have become the focus of prevention. Those efforts target holistic development of children, and aim to teach that taking care of one's mental health is equally normal to taking care of one's physical well-being. The reported qualitative data in this dissertation showed that about half of young people made future oriented choices, e.g., not consuming alcohol, and prioritizing studying to gain access to secondary education. However, roughly the other half of this sample appears in need of support which could be provided through adapting and implementing social-emotional learning (SEL) curricula (see e.g., Durlak et al., 2011). Such SEL programs are effective in increasing youth's emotion recognition, stress management, empathy, problem-solving and decision-making skills (Durlak et al., 2011).

\section{Activities and products}

This dissertation will be disseminated to school organizations, teachers, community leaders, individuals, and translators. Scientific articles resulting from the chapters of this dissertation have been and will be published full open access, allowing a wide audience to access the findings. Local discussions will be initiated and facilitated by the author of this dissertation, to connect people and share knowledge and experiences. These discussions will mainly take place electronically given large distances. We will ensure that the different geographical regions are contacted, thus creating the possibility for grass root effects.

Further research in evidence-based approaches that target the identified areas of interventions will be undertaken (e.g., motivational interviewing). Some community leaders had already proposed community based group activities in order to start talking about life's challenges and feelings. Motivational Interviewing (MI) appears especially appropriate in the ever-changing economic-political situation and uncertainty. One of the core elements concerns discussing ambiguity toward behavioral change, which is a general challenge for humans but may be a particular issue in demanding situations. Last, MI has been shown to reduce barriers to seeking help. Ultimately, the findings from this dissertation as well as the community group meetings could result in a trainer's guide to facilitate such discussions.

\section{Innovation}


This study is innovative in its combination of both interdisciplinary research and mixed methods. First, taking both neuropsychological development as well as social (health) psychology into account has resulted in a more holistic view on children's cognitive development. Second, combining quantitative and qualitative research methods supported a richer description of cognitive functions and environmental variables. Conducting the research in itself evoked questions and discussions with the target group(s): Teachers asked what tests entailed and how that related to learning and development of children. Discussions about differences in cultures between participants and researchers moved away from general educational complaints toward collegial consultation. The qualitative data provided participants a platform to share their stories and additionally, it appeared that some started to reflect, which may be a possible next step in individual processes to change behavior or engage in community (prevention) activities. Last, EFs have been implicated in a range of topics related to development, cognition, health and well-being (Eisenburg et al., 2019). Curricular adaptations relating to mental health and holistic development, activities such as developing self-efficacy and coping skills for practical life situations, have been identified in other countries as beneficial to children's health and general development. This study provides additional ideas how to incorporate unique social and cultural characteristics of a population in such curricula targeting general mental health.

\section{Implementation}

To disseminate our findings among organizations publishing (established) norms and populations that differ from generally established test norms, we will seek further publication and continue to draw attention to the dissemination that has already taken place (conference presentations and open access publications) on professional social media (e.g., LinkedIn). Importantly, plans to develop a trainer's guide to facilitate motivational interviewing in culturally and socially appropriate manners are being developed. Last, dissemination to different stakeholders mentioned above has started electronically. 


\section{References}

Aliyev, H. (2019). The Logic of Ethnic Responsibility and Pro-government Mobilization in East Ukraine Conflict. Comparative Political Studies, 0010414019830730.

Alloway, T. P., \& Alloway, R. G. (2010). Investigating the predictive roles of working memory and IQ in academic attainment. Journal of Experimental Child Psychology, 106(1), 20-29.

Alloway, T. P., Gathercole, S. E., \& Pickering, S. J. (2006). Verbal and visuospatial short-term and working memory in children: Are they separable? Child development, 77(6), 16981716.

Alloway, T. P., Gathercole, S. E., Willis, C., \& Adams, A. M. (2004). A structural analysis of working memory and related cognitive skills in young children. Journal of Experimental Child Psychology, 87(2), 85-106.

Alloway, T. P., \& Passolunghi, M. C. (2011). The relationship between working memory, IQ, and mathematical skills in children. Learning and Individual Differences, 21(1), 133-137.

Alonso, J., Buron, A., Bruffaerts, R., He, Y., Posada-Villa, J., Lepine, J. P., \& Mneimneh, Z. N. (2008). Association of perceived stigma and mood and anxiety disorders: results from the World Mental Health Surveys. Acta Psychiatrica Scandinavica, 118(4), 305314.

American Psychiatric Association. (2013). Diagnostic and statistical manual of mental disorders (DSM-5®). American Psychiatric Pub.

Anderson, C. (2010). Presenting and evaluating qualitative research. American journal of pharmaceutical education, 74(8), 141.

Anderson, P. (2002). Assessment and Development of Executive Function (EF) During Childhood. Child Neuropsychology, 8(2), 71-82. doi:10.1076/chin.8.2.71.8724

Angermeyer, M. C., Matschinger, H., \& Schomerus, G. (2013). Attitudes towards psychiatric treatment and people with mental illness: changes over two decades. The British Journal of Psychiatry, bjp-bp.

Ardila, A. (1995). Directions of research in cross-cultural neuropsychology. Journal of Clinical and Experimental Neuropsychology, 17(1), 143-150.

Ardila, A. (1996). Towards a cross-cultural neuropsychology. Journal of Social and Evolutionary Systems, 19(3), 237-248.

Ardila, A. (2005). Cultural Values Underlying Psychometric Cognitive Testing. Neuropsychology Review, 15(4), 185.

Ardila, A., Rosselli, M., Matute, E., \& Guajardo, S. (2005). The Influence of the Parents' Educational Level on the Development of Executive Functions. Developmental Neuropsychology, 28(1), 539-560.

Ardila, A., Rosselli, M., Matute, E., \& Inozemtseva, O. (2011). Gender differences in cognitive development. Developmental Psychology, 47(4), 984.

Baddeley, A. D., Allen, R. J., \& Hitch, G. J. (2011). Binding in visual working memory: The role of the episodic buffer. Neuropsychologia, 49(6), 1393-1400. doi:10.1016/j.neuropsychologia.2010.12.042. 
Baer, J., Kutner, M., Sabatini, J., \& White, S. (2009). Basic Reading Skills and the Literacy of America's Least Literate Adults: Results from the 2003 National Assessment of Adult Literacy (NAAL) Supplemental Studies. NCES 2009-481. National Center for Education Statistics.

Bailey, C. E. (2007). Cognitive accuracy and intelligent executive function in the brain and in business. Annals of the New York Academy of Sciences, 1118(1), 122-141.

Bakhireva, L. N., Wilsnack, S. C., Kristjanson, A., Yevtushok, L., Onishenko, S., Wertelecki, W., \& Chambers, C. D. (2011). Paternal drinking, intimate relationship quality, and alcohol consumption in pregnant Ukrainian women. Journal of studies on alcohol and drugs, 72(4), 536-544.

Balogun, O., Koyanagi, A., Stickley, A., Gilmour, S., \& Shibuya, K. (2014). Alcohol consumption and psychological distress in adolescents: a multi-country study. Journal of Adolescent Health, 54(2), 228-234. doi:10.1016/j.jadohealth.2013.07.034.

Barnow, S., Schuckit, M. A., Lucht, M., John, U., \& Freyberger, H. J. (2002). The importance of a positive family history of alcoholism, parental rejection and emotional warmth, behavioral problems and peer substance use for alcohol problems in teenagers: a path analysis. Journal of Studies on Alcohol and Drugs, 63(3), 305-315. doi:10.15288/jsa.2002.63.305.

Barona, A., Reynolds, C. R., \& Chastain, R. (1984). A demographically based index of premorbid intelligence for the WAIS-R. Journal of Consulting and Clinical Psychology, 52(5), 885.

Bartholomew Eldredge, L. K., Markham, C. M., Ruiter, R. A., Fernández, M. E., Kok, G., \& Parcel, G. S. (2016). Planning health promotion programs: an intervention mapping approach.

Batson, C. D., \& Ahmad, N. Y. (2009). Using empathy to improve intergroup attitudes and relations. Social Issues and Policy Review, 3(1), 141-177.

Bauer, P. M., Hanson, J. L., Pierson, R. K., Davidson, R. J., \& Pollak, S. D. (2009). Cerebellar volume and cognitive functioning in children who experienced early deprivation. Biological psychiatry, 66(12), 1100-1106.

Bechger, T., Hemker, B., \& Maris, G. (2009). <cito_pok_memorandum_2009-01-3.pdf>.

Berlach, N., Kulyk, O., \& Losych, S. (2019). Illegally-Obtained Income As A Structural Component Of Shadow Processes In The Economy. Baltic Journal of Economic Studies, 4(5), 26-30.

Biernacki, P., \& Waldorf, D. (1981). Snowball sampling: Problems and techniques of chain referral sampling. Sociological methods \& research, 10(2), 141-163.

Bigelow, J., \& Poremba, A. (2012). Comparing short-term memory among sensory modalities. Visual Cognition, 20(9), 1012-1016.

Bos, K. J., Fox, N., Zeanah, C. H., \& Nelson, C. A. (2009). Effects of early psychosocial deprivation on the development of memory and executive function. Frontiers in Behavioral Neuroscience, 3, 16.

Bos, A. E. R., Pryor, J. B., Reeder, G. D., \& Stutterheim, S. E. (2013). Stigma: Advances in Theory and Research. Basic and Applied Social Psychology, 35(1), 1-9. doi:10.1080/01973533.2012.746147.

Bos, A. E., Schaalma, H. P., \& Pryor, J. B. (2008). Reducing AIDS-related stigma in developing countries: The importance of theory-and evidence-based interventions. Psychology, Health \& Medicine, 13(4), 450-460.

Bradley, R. H., \& Corwyn, R. F. (2002). Socioeconomic status and child development. Annual review of psychology, 53(1), 371-399. 
Braun, V., \& Clarke, V. (2006). Using thematic analysis in psychology. Qualitative research in psychology, 3(2), 77-101. doi:10.1191/1478088706qp063oa

Britton, P. C., Patrick, H., Wenzel, A., \& Williams, G. C. (2011). Integrating motivational interviewing and self-determination theory with cognitive behavioral therapy to prevent suicide. Cognitive and Behavioral Practice, 18(1), 16-27.

Brocki, K. C., \& Bohlin, G. (2004). Executive Functions in Children Aged 6 to 13: A Dimensional and Developmental Study. Developmental Neuropsychology, 26(2), 571-593. doi:10.1207/s15326942dn2602_3.

Bromet, E. J., Gluzman, S. F., Paniotto, V. I., Webb, C. P., Tintle, N. L., Zakhozha, V., ... \& Schwartz, J. E. (2005). Epidemiology of psychiatric and alcohol disorders in Ukraine. Social psychiatry and psychiatric epidemiology, 40(9), 681-690.

Bronfenbrenner, U. (1994). Ecological models of human development. International encyclopedia of education, 3(2), 37-43.

Bronfenbrenner, U., \& Morris, P. A. (2006). The bioecological model of human development. In R. M. Lerner \& W. Damon (Eds.), Handbook of child psychology: Theoretical models of human development (pp. 793-828). Hoboken, NJ, US: John Wiley \& Sons Inc.

Burk, J. P., \& Sher, K. J. (1990). Labeling the child of an alcoholic: negative stereotyping by mental health professionals and peers. Journal of Studies on Alcohol, 51(2), 156-163.

Burlaka, V., Grogan-Kaylor, A., Savchuk, O., \& Graham-Bermann, S. A. (2017). The relationship between family, parent, and child characteristics and intimate-partner violence (IPV) among Ukrainian mothers. Psychology of violence, 7(3), 469.

Cambridge Cognition (2012). Cambridge Neuropsychological Test Automated Battery $\left(\right.$ CANTABeclipse $\left.^{\circledR}\right)$ manual. Cambridge: Cambridge Cognition ltd.

Cambridge Cognition (2014). Child Norms Cambridge Neuropsychological Test Automated Battery (Manual). Cambridge: Cambridge Cognition ltd.

Cambridge Cognition (2019). Cambridge Neuropsychological Test Automated Battery. Retrieved from https://www.cambridgecognition.com/

Carman, C. A., \& Taylor, D. K. (2010). Socioeconomic status effects on using the Naglieri Nonverbal Ability Test (NNAT) to identify the gifted/talented. Gifted Child Quarterly, 54(2), 75-84. Chambers, C. D., Yevtushok, L., Zymak-Zakutnya, N., Korzhynskyy, Y., Ostapchuk, L., Akhmedzhanova, D., ... Wertelecki, W. (2014). Prevalence and Predictors of Maternal Alcohol Consumption in Two Regions of Ukraine. Alcohol, Clinical and Experimental Research, 38(4), 1012-1019.

Chevignard, M. P., Catroppa, C., Galvin, J., \& Anderson, V. (2010). Development and evaluation of an ecological task to assess executive functioning post childhood TBI: The Children's Cooking Task. Brain Impairment, 11(2), 125-143.

Cialdini, R. B., Reno, R. R., \& Kallgren, C. A. (1990). A focus theory of normative conduct: Recycling the concept of norms to reduce littering in public places. Journal of personality and social psychology, 58(6), 1015.

Chien, Y. L., Gau, S. S., Shang, C. Y., Chiu, Y. N., Tsai, W. C., \& Wu, Y. Y. (2015). Visual memory and sustained attention impairment in youths with autism spectrum disorders. Psychological Medicine, 45(11), 2263-2273. doi:10.1017/S0033291714003201.

Cherner, M., Suarez, P., Lazzaretto, D., i Fortuny, L. A., Mindt, M. R., Dawes, S., ... \& HNRC group. (2007). Demographically corrected norms for the Brief Visuospatial Memory Test- 
revised and Hopkins Verbal Learning Test-revised in Monolingual Spanish speakers from the US-Mexico border region. Archives of Clinical Neuropsychology, 22(3), 343-353.

Clement, S., Schauman, O., Graham, T., Maggioni, F., Evans-Lacko, S., Bezborodovs, N., ... \& Thornicroft, G. (2015). What is the impact of mental health-related stigma on help-seeking? A systematic review of quantitative and qualitative studies. Psychological medicine, 45(1), $11-27$.

Cloninger, C., Svrakic, D. M., \& Przybeck, T. R. (1993). A psychobiological model of temperament and character. Archives of General Psychiatry, 50(12), 975-990. doi:10.1001/archpsyc.1993.01820240059008

Corrigan, P. W., \& Kosyluk, K. A. (2013). Erasing the stigma: Where science meets advocacy. Basic and applied social psychology,35(1), 131-140.

Corrigan, P. W., \& Kosyluk, K. A. (2014). Mental illness stigma: Types, constructs, and vehicles for change.

Corrigan, P. W., \& Miller, F. E. (2004). Shame, blame, and contamination: A review of the impact of mental illness stigma on family members. Journal of Mental Health, 13(6), 537-548.

Corrigan, P. W., \& Shapiro, J. R. (2010). Measuring the impact of programs that challenge the public stigma of mental illness. Clinical Psychology Review, 30(8), 907-922. doi:10.1016/j.cpr.2010.06.004.

Corrigan, P. W., \& Watson, A. C. (2002). The paradox of self-stigma and mental illness. Clinical Psychology: Science and Practice, 9(1), 35- 53.

Courtney, S. M., Ungerleider, L. G., Keil, K., \& Haxby, J. V. (1996). Object and spatial visual working memory activate separate neural systems in human cortex. Cerebral cortex, 6(1), 39-49.

Crescentini, C., Seyed-Allaei, S., Vallesi, A., \& Shallice, T. (2012). Two networks involved in producing and realizing plans. Neuropsychologia, 50(7), 1521-1535. doi.org/10.1016/j.neuropsychologia.2012.03.005.

Crisp, A., Gelder, M., Goddard, E., \& Meltzer, H. (2005). Stigmatization of people with mental illnesses: a follow-up study within the Changing Minds campaign of the Royal College of Psychiatrists. World psychiatry, 4(2), 106.

Crocker, J., \& Major, B. (1989). Social stigma and self-esteem: The self-protective properties of stigma. Psychological Review, 96(4), 608-630. doi:10.1037/0033-295X.96.4.608

Currie, C., Roberts, C., Morgan, A., Smith, R., Setterbulte, W., Samdel, O., et al. (Eds.) (2004). Young people's health in context; Health Behaviour in School-aged Children (HBSC) Study: International report from the 2001/2002 survey. Retrieved from http://www.hbsc.org/ downloads/IntReport04/Part1.pdf.

Dabbagh, N., \& Kitsantas, A. (2005). Using web-based pedagogical tools as scaffolds for selfregulated learning. Instructional Science, 33(5-6), 513-540.

D'anieri, P. (2018). Politics and society in Ukraine. New York, USA: Routledge.

Davidson, M. C., Amso, D., Anderson, L. C., \& Diamond, A. (2006). Development of cognitive control and executive functions from 4 to 13 years: Evidence from manipulations of memory, inhibition, and task switching. Neuropsychologia, 44(11), 2037-2078.

Davis-Kean, P. E. (2005). The influence of parent education and family income on child achievement: the indirect role of parental expectations and the home environment. Journal of family psychology, 19(2), 294. 
De Bolle, M., De Fruyt, F., McCrae, R. R., Löckenhoff, C. E., Costa, P. T., Aguilar-Vafaie, M. E., . . . Terracciano, A. (2015). The Emergence of Sex Differences in Personality Traits in Early Adolescence: A Cross-Sectional, Cross-Cultural Study. Journal of personality and social psychology, 108(1), 171-185. doi:10.1037/a0038497.

Deci, E. L., \& Ryan, R. M. (2008). Self-determination theory: A macrotheory of human motivation, development, and health. Canadian psychology/Psychologie Canadienne, 49(3), 182.

De Graaf, T. (2016). Attention: Phenomena, forms, and theories of attention (pp. 1-20). Maastricht: Maastricht University.

De Luca, C. R., Wood, . S. J., Anderson, V., Buchanan, J. A., Proffitt, T. M., Mahony, K., \& Pantelis, C. (2003). Normative Data From the Cantab. I: Development of Executive Function Over the Lifespan. Journal of Clinical \& Experimental Neuropsychology, 25(2), 242.

Devries, K. M., Child, J. C., Bacchus, L. J., Mak, J., Falder, G., Graham, K., ... \& Heise, L. (2014). Intimate partner violence victimization and alcohol consumption in women: a systematic review and meta-analysis. Addiction, 109(3), 379-391.

Diamond, A. (2002). Normal development of prefrontal cortex from birth to young adulthood: Cognitive functions, anatomy, and biochemistry. Principles of frontal lobe function, 466503 .

Diamond, A. (2012). Activities and programs that improve children's executive functions. Current directions in psychological science, 21(5), 335-341.

Diamond, A. (2013). Executive functions. Annual Review of Psychology, 64, 135-168. doi:10.1146/annurev-psych-113011-143750.

Diamond, A., \& Lee, K. (2011). Interventions shown to aid executive function development in children 4 to 12 years old. Science, 333(6045), 959-964.

Dijker, A. J., \& Koomen, W. (2003). Extending Weiner's Attribution-Emotion Model of Stigmatization of Ill Persons. Basic and Applied Social Psychology, 25(1), 51-68. doi:10.1207/S15324834BASP2501_4.

Donovan, J. E., \& Molina, B. S. G. (2008). Children's Introduction to Alcohol Use: Sips and Tastes. Alcohol, Clinical and Experimental Research, 32(1), 108-119. doi:10.1111/j.15300277.2007.00565.x

Donovan, J. E., \& Molina, B. S. G. (2011). Childhood Risk Factors for Early-Onset Drinking. Journal of Studies on Alcohol and Drugs, 72(5), 741-751.

Duncan, G. J., Dowsett, C. J., Claessens, A., Magnuson, K., Huston, A. C., Klebanov, P., . . BrooksGunn, J. (2007). School readiness and later achievement. Developmental Psychology, 43(6), 1428 .

Durlak, J. A., Weissberg, R. P., Dymnicki, A. B., Taylor, R. D., \& Schellinger, K. B. (2011). The impact of enhancing students' social and emotional learning: A meta-analysis of school-based universal interventions. Child development, 82(1), 405-432.

Earnshaw, V. A., Smith, L. R., Cunningham, C. O., \& Copenhaver, M. M. (2015). Intersectionality of internalized HIV stigma and internalized substance use stigma: Implications for depressive symptoms. Journal of Health Psychology, 20, 1083-1089.

Eisenberg, I. W., Bissett, P. G., Enkavi, A. Z., Li, J., MacKinnon, D. P., Marsch, L. A., \& Poldrack, R. A. (2019). Uncovering the structure of self-regulation through data-driven ontology discovery. Nature communications, 10(1), 2319. 
Elliott, R., Sahakian, B. J., McKay, A. P., Herrod, J. J., Robbins, T. W., \& Paykel, E. S. (1996). Neuropsychological impairments in unipolar Depression: the influence of perceived failure on subsequent performance. Psychological medicine, 26(5), 975-989.

Farrell Pagulayan, K., Busch, R. M., Medina, K. L., Bartok, J. A., \& Krikorian, R. (2006). Developmental Normative Data for the Corsi Block-Tapping Task. Journal of Clinical and Experimental Neuropsychology, 28(6), 1043-1052. doi:10.1080/13803390500350977.

Fazeli, P. L., Ross, L. A., Vance, D. E., \& Ball, K. (2013). The Relationship Between Computer Experience and Computerized Cognitive Test Performance Among Older Adults. The Journals of Gerontology Series B: Psychological Sciences and Social Sciences, 68(3), 337346.

Field, A. (2009). Discovering statistics using SPSS. Sage publications.

Fiorillo, D., \& Sabatini, F. (2011). Quality and quantity: the role of social interactions in self-reported individual health. Social Science \& Medicine, 73(11), 1644-1652.

Fishbein, M. \& Ajzen, I. (2010). Introduction. Predicting and changing behavior: The Reasoned Action Approach (p. 2 - 28). New York: Taylor \& Francis.

Fisher, S. D., Gray, J. P., Black, M. J., Davies, J. R., Bednark, J. G., Redgrave, P., . . Reynolds, J. N. (2014). A behavioral task for investigating action discovery, selection and switching: comparison between types of reinforcer. Frontiers in Behavioral Neuroscience, 8, 398. doi:10.3389/fnbeh.2014.00398.

Frazier Norbury, C., \& Sparks, A. (2012). Difference or Disorder? Cultural Issues in Understanding Neurodevelopmental Disorders (Vol. 49).

Fried, R., Hirshfeld-Becker, D., Petty, C., Batchelder, H., \& Biederman, J. (2015). How informative is the CANTAB to assess executive functioning in children with ADHD? A controlled study. Journal of Attention Disorders, 19(6), 468-475.

Fuster, J. M. (2002). Physiology of executive functions: The perception-action cycle. Principles of frontal lobe function, 96108.

Galsworthy, M. J., Dionne, G., Dale, P. S., \& Plomin, R. (2000). Sex differences in early verbal and non-verbal cognitive development. Developmental Science, 3(2), 206-215.

Garon, N., Bryson, S. E., \& Smith, I. M. (2008). Executive function in preschoolers: a review using an integrative framework. Psychological bulletin, 134(1), 31.

Gathercole, S. E., Pickering, S. J., Ambridge, B., \& Wearing, H. (2004). The structure of working memory from 4 to 15 years of age. Developmental Psychology, 40(2), 177.

Geary, D. C. (2013). Early foundations for mathematics learning and their relations to learning disabilities. Current Directions in Psychological Science, 22(1), 23-27.

Geier, C. F., Garver, K., Terwilliger, R., \& Luna, B. (2009). Development of working memory maintenance. Journal of Neurophysiology, 101(1), 84-99. doi:10.1152/jn.90562.2008.

Giessman, J. A., Gambrell, J. L., \& Stebbins, M. S. (2013). Minority performance on the Naglieri Nonverbal Ability Test, Second Edition, versus the Cognitive Abilities Test, Form 6: One gifted program's experience. Gifted Child Quarterly, 57(2), 101-109. doi:10.1177/0016986213477190.

Gluck, M. A., Mercado, E., \& Meyers, C. E. (2008). Learning and memory: From brain to behaviour: Worth Publishers, NY.

Goldstein, E. B. (2002). Appendix A: Signal detection: Procedure and theory. Sensation and perception $\left(6^{\text {th }} \mathrm{ed}\right)$, p. 583-590. USA: Cengage Learning. 
Goncharuk, A. G. (2009). Improving of the efficiency through benchmarking: a case of Ukrainian breweries. Benchmarking: an international journal, 16(1), 70-87.

Green, R., Till, C., Al-Hakeem, H., Cribbie, R., Téllez-Rojo, M. M., Osorio, E., ... \& Schnaas, L. (2019). Assessment of neuropsychological performance in Mexico City youth using the Cambridge Neuropsychological Test Automated Battery (CANTAB). Journal of clinical and experimental neuropsychology, 41(3), 246-256.

Gruhn, W., \& Rauscher, F. (2002). The neurobiology of music cognition and learning. The new handbook of research on music teaching and learning, 445-460. Retrieved from http://www.posgrado.unam.mx/musica/lecturas/educacion/Neurobiology\%20of\%learning. pdf

Gur, R. C., Richard, J., Calkins, M. E., Chiavacci, R., Hansen, J. A., Bilker, W. B., ... \& AbouSleiman, P. M. (2012). Age group and sex differences in performance on a computerized neurocognitive battery in children age 8-21. Neuropsychology, 26(2), 251.

Hackman, D. A., Betancourt, L. M., Gallop, R., Romer, D., Brodsky, N. L., Hurt, H., \& Farah, M. J. (2014). Mapping the trajectory of socioeconomic disparity in working memory: Parental and neighborhood factors. Child development, 85(4), 1433-1445.

Hackman, D. A., \& Farah, M. J. (2009). Socioeconomic status and the developing brain. Trends in cognitive sciences, 13(2), 65-73.

Hammers, D., Michalski, L., Reese, E., Persad, C., Wilson, S., Powells, D., . . Giordani, B. (2011). Using the CANTAB computerized battery to discriminate mild cognitive impairment and dementias. Alzheimer's \& Dementia, 7(4), S537.

Hasin, D. S., Stinson, F. S., Ogburn, E., \& Grant, B. F. (2007). Prevalence, correlates, disability, and comorbidity of DSM-IV alcohol abuse and dependence in the United States: results from the National Epidemiologic Survey on Alcohol and Related Conditions. Archives of general psychiatry, 64(7), 830-842.

Hatzenbuehler, M. L., Phelan, J. C., \& Link, B. G. (2013). Stigma as a fundamental cause of population health inequalities. American journal of public health, 103(5), 813-821.

Haukkala, H. (2015). From cooperative to contested Europe? The conflict in Ukraine as a culmination of a long-term crisis in EU-Russia relations. Journal of Contemporary European Studies, 23(1), 25-40.

Haverfield, M. C., \& Theiss, J. A. (2016). Parent's alcoholism severity and family topic avoidance about alcohol as predictors of perceived stigma among adult children of alcoholics: Implications for emotional and psychological resilience. Health communication, 31(5), 606616.

Havnes, A., Smith, K., Dysthe, O., \& Ludvigsen, K. (2012). Formative assessment and feedback: Making learning visible. Studies in Educational Evaluation, 38(1), 21-27. doi.org/10.1016/j.stueduc.2012.04.001.

Hawkins, J. D., Kosterman, R., Catalano, R. F., Hill, K. G., \& Abbott, R. D. (2005). Promoting positive adult functioning through social development intervention in childhood: Long-term effects from the Seattle Social Development Project. Archives of Pediatrics \& Adolescent Medicine, 159(1), 25-31.

Heaton, R. K., Taylor, M. J., \& Manly, J. (2003). Chapter 5 - Demographic Effects and Use of Demographically Corrected Norms with the WAIS-III and WMS-III Clinical Interpretation of the WAIS-III and WMS-III (pp. 181-210). San Diego: Academic Press. 
Hellemans, K. G., Sliwowska, J. H., Verma, P., \& Weinberg, J. (2010). Prenatal alcohol exposure: fetal programming and later life vulnerability to stress, depression and anxiety disorders. Neuroscience \& Biobehavioral Reviews, 34(6), 791-807.

Hellman, J. S., Jones, G., \& Kaufmann, D. (2003). Seize the state, seize the day: state capture and influence in transition economies. Journal of comparative economics, 31(4), 751-773.

Herrick, C. (2012). The political ecology of alcohol as "disaster" in South Africa's Western Cape. Geoforum, 43(6), 1045-1056.

Hess, A. E. M., Frohlich, T. C., Calio, V. (2014). The Heaviest-Drinking Countries in the World. Retrieved from http://247wallst.com/special-report/2014/05/15/the-heaviest-drinkingcountries-in-the-world/

Hession, C. (2012). Alcohol use: from childhood through adolescence. Journal of pediatric nursing, 27(5), e50-e58. doi:10.1016/j.pedn.2012.01.003

Hingson, R. W., Heeren, T., \& Winter, M. R. (2006). Age at drinking onset and alcohol dependence: age at onset, duration, and severity. Archives of pediatrics \& adolescent medicine, 160(7), 739-746.

Hitch, G. J., Towse, J. N., \& Hutton, U. (2001). What limits children's working memory span? Theoretical accounts and applications for scholastic development. Journal of Experimental Psychology: General, 130(2), 184.

Huang, A. S., Klein, D. N., \& Leung, H. C. (2016). Load-related brain activation predicts spatial working memory performance in youth aged 9-12 and is associated with executive function at earlier ages. Developmental Cognitive Neuroscience, 17, 1-9. doi:http://dx.doi.org/10.1016/j.den.2015.10.007.

Hughes, C. (2011). Changes and challenges in 20 years of research into the development of executive functions. Infant and Child Development, 20(3), 251-271.

Hughes, C. H., \& Ensor, R. A. (2009). How do families help or hinder the emergence of early executive function?. New directions for child and adolescent development, 2009(123), 3550 .

Hurks, P., \& Hendriksen, J. (2015). WPPSI-III-NL Intelligentiemeting bij kinderen en adolescenten. In Resing, W.C.M. (Ed), Handboek intelligentietheorie en testgebruik (113-140). Amsterdam: Pearson.

Hurlstone, M. J., Hitch, G. J., \& Baddeley, A. D. (2014). Memory for serial order across domains: An overview of the literature and directions for future research. Psychological Bulletin, 140(2), 339.

Jackson, P. (2014). Ukraine crisis: 'Frozen conflicts' and the Kremlin. Retrieved from BBC news website: http://www.bbc.com/news/world-europe-29078541.

Jackson, C., Ennett, S. T., Dickinson, D. M., \& Bowling, J. M. (2013). Attributes that differentiate children who sip alcohol from abstinent peers. Journal of Youth Adolescence, 42(11), $1687-$ 1695. doi:10.1007/s10964-012-9870-8.

Jacob, T., Moser, R. P., Windle, M., Loeber, R., \& Stouthamer-Loeber, M. (2000). A new measure of parenting practices involving preadolescent-and adolescent-aged children. Behavior modification, 24(5), 611-634.

Jacob, K.S., Sharan, P., Mirza, I., Garrido-Cumbrera, M., Seedat, S., Mari, J.J., Sreenivas, V., \& Saxena, S. (2007). Mental health systems in countries, where are we now? The Lancet, 370 , 9592, 1061-1077. 
Jacobus, J., \& Tapert, S. F. (2013). Neurotoxic Effects of Alcohol in Adolescence. Annual Review of Clinical Psychology, 9(1), 703-721. doi:10.1146/annurev-clinpsy-050212-185610.

Jensen, C. D., Cushing, C. C., Aylward, B. S., Craig, J. T., Sorell, D. M., \& Steele, R. G. (2011). Effectiveness of motivational interviewing interventions for adolescent substance use $\mathrm{b}$ behavior change: a meta-analytic review. Journal of consulting and clinical psychology, 79(4), 433.

Jiang, Y., Ekono, M., Skinner, C. (2016). Basic Facts about Low-income Children: Children Aged 6 through 11 Years, 2014. National Center for Children in Poverty, Columbia University Mailman School of Public Health. Retrieved from http://www.nccp.org/publications/pub_1146.html.

Johnson, M. H., Mareschal, D., \& Csibra, G. (2008). The development and integration of the dorsal and ventral visual pathways in object processing. In Nelson, C. A., \& Luciana, M. (Eds). Handbook of developmental cognitive neuroscience (467-478). Cambridge MA: MIT press.

Jones, L., Bates, G., McCoy, E., \& Bellis, M. A. (2015). Relationship between alcohol-attributable disease and socioeconomic status, and the role of alcohol consumption in this relationship: a systematic review and meta-analysis. BMC Public Health, 15(1), 400.

Jones, S. M., \& Bouffard, S. M. (2012). Social and Emotional Learning in Schools: From Programs to Strategies. Social Policy Report. Volume 26, Number 4. Society for Research in Child Development.

Kaplan, G. A., Turrell, G., Lynch, J. W., Everson, S. A., Helkala, E.-L., \& Salonen, J. T. (2001). Childhood socioeconomic position and cognitive function in adulthood. International Journal of Epidemiology, 30(2), 256-263.

Karmiloff-Smith, A., Scerif, G., \& Ansari, D. (2003). Double dissociations in developmental disorders? Theoretically misconceived, empirically dubious. Cortex, 39(1), 161-163.

Kessels, R. P., van Zandvoort, M. J., Postma, A., Kappelle, L. J., \& de Haan, E. H. (2000). The Corsi Block-Tapping Task: standardization and normative data. Applied Neuropsychology, 7(4), 252-258. doi:10.1207/s15324826an0704_8.

Keyes, K., Hatzenbuehler, M., McLaughlin, K., Link, B., Olfson, M., Grant, B., \& Hasin, D. (2010). Stigma and treatment for alcohol disorders in the United States. American journal of epidemiology, 172(12), 1364-1372.

Klenberg, L., Korkman, M., \& Lahti-Nuuttila, P. (2001). Differential Development of Attention and Executive Functions in 3- to 12-Year-Old Finnish Children. Developmental Neuropsychology, 20(1), 407-428. doi:10.1207/S15326942DN2001_6.

Koball, H. \& Jiang, Y. (2018). Basic Facts about Low-income Children: Children under 18 Years, 2016. National Center for Children in Poverty, Columbia University Mailman School of Public Health. Retrieved from http://www.nccp.org/publications/pub_1194.htm.

Kodituwakku, P. W. (2009). Neurocognitive profile in children with fetal alcohol spectrum disorders. Developmental Disabilities Research Review, 15(3), 218-224. doi:10.1002/ddrr.73.

Korkman, M., Kemp, S. L., \& Kirk, U. (2001). Effects of age on neurocognitive measures of children ages 5 to 12: A cross-sectional study on 800 children from the United States. Developmental neuropsychology, 20(1), 331-354.

Kravitz, D. J., Saleem, K. S., Baker, C. I., Ungerleider, L. G., \& Mishkin, M. (2013). The ventral visual pathway: an expanded neural framework for the processing of object quality. Trends in cognitive sciences, 17(1), 26-49.

Kubicek, P. (2002). Civil society, trade unions and post-soviet democratisation: Evidence from Russia and Ukraine. Europe-Asia Studies, 54(4), 603-624. 
Lachenmeier, D. W., \& Sohnius, E.-M. (2008). The role of acetaldehyde outside ethanol metabolism in the carcinogenicity of alcoholic beverages: evidence from a large chemical survey. Food and chemical toxicology, 46(8), 2903-2911.

Lachenmeier, D. W., Taylor, B. J., \& Rehm, J. (2011). Alcohol under the radar: Do we have policy options regarding unrecorded alcohol? International Journal of Drug Policy, 22(2), 153 160.

Landberg, J. (2008). Alcohol and suicide in Eastern Europe. Drug and Alcohol Review, 27(4), 361 373.

Lange, R. T., Chelune, G. J., Taylor, M. J., Woodward, T. S., \& Heaton, R. K. (2006). Development of demographic norms for four new WAIS-III/WMS-III indexes. Psychological Assessment, $18(2), 174$.

Laerd Statistics (2015). Statistical tutorials and software guides. Retrieved from https://statistics.laerd.com/

Lecerf, T., \& De Ribaupierre, A. (2005). Recognition in a visuospatial memory task: The effect of presentation. European Journal of Cognitive Psychology, 17(1), 47-75

Lehto, J. E., Juujärvi, P., Kooistra, L., \& Pulkkinen, L. (2003). Dimensions of executive functioning: Evidence from children. British Journal of Developmental Psychology, 21(1), 59-80.

Leng, X., \& Shaw, G. L. (1991). Toward a neural theory of higher brain function using music as a window. World Scientific Publishing Company.

Lekhan, V., Rudiy, V., \& Richardson, E. (2010). Ukraine Health system review. Health Systems in T Transition, 12(8). Retrieved from http://www.euro.who.int/_data/assets/pdf_file/0010/140599/e94973.pdf. Accessed 21 September 2019.

León, I., Cimadevilla, J. M., \& Tascón, L. (2014). Developmental gender differences in children in a virtual spatial memory task. Neuropsychology, 28(4), 485-495. doi:10.1037/neu0000054

Lewis, T. P., \& Hession, C. (2012). Alcohol use: from childhood through adolescence. Journal of pediatric nursing, 27(5), e50-8.

Lezak, M. D., Howieson, D. B., Bigler, E. D., \& Tranel, D. (2012). Neuropsychological Assessment. New York: Oxford University Press.

Link, B. G., \& Phelan, J. (2014). Stigma power. Social Science \& Medicine, 103, 24-32.

Link, B. G., Yang, L. H., Phelan, J. C., \& Collins, P. Y. (2004). Measuring mental illness stigma. Schizophrenia bulletin, 30(3), 511-541.

Logie, R. H., \& Marchetti, C. (1991). Visuo-spatial working memory: Visual, spatial or central executive. Mental images in human cognition, 80, 105Á.

Lohman, D. F., \& Gambrell, J. L. (2012). Using nonverbal tests to help identify academically talented children. Journal of Psychoeducational Assessment, 30(1), 25-44.

Lowe, C., \& Rabbitt, P. (1998). Testlre-test reliability of the CANTAB and ISPOCD neuropsychological batteries: theoretical and practical issues. Neuropsychologia, 36(9), 915-923.

Luciana, M. (2003). Practitioner review: computerized assessment of neuropsychological function in children: clinical and research applications of the Cambridge Neuropsychological Testing Automated Battery (CANTAB). Journal of Child Psychology and Psychiatry, 44(5), 649663. 
Luciana, M., Collins, P. F., Muetzel, R. L., \& Lim, K. O. (2013). Effects of alcohol use initiation on brain structure in typically developing adolescents. American Journal of Drug and Alcohol Abuse, 39(6), 345-355. doi:10.3109/00952990.2013.837057

Luciana, M., Conklin, H. M., Hooper, C. J., \& Yarger, R. S. (2005). The development of nonverbal working memory and executive control processes in adolescents. Child Development, 76(3), 697-712.

Luciana, M., \& Nelson, C. A. (1998). The functional emergence of prefrontally-guided working memory systems in four-to eight-year-old children. Neuropsychologia, 36(3), 273-293.

Luciana, M., \& Nelson, C. A. (2000). Neurodevelopmental assessment of cognitive function using the Cambridge Neuropsychological Testing Automated Battery (CANTAB): validation and future goals. Functional Neuroimaging in Child Psychiatry. Cambridge University Press, Cambridge, 379-397.

Luciana, M., \& Nelson, C.A. (2002). Assessment of neuropsychological function through use of the Cambridge Neuropsychological Testing Automated Battery: performance in 4-to 12-yearold children. Developmental Neuropsychology, 22(3), 595-624.

Luck, S. J., \& Vogel, E. K. (2013). Visual working memory capacity: from psychophysics and neurobiology to individual differences. Trends in cognitive sciences, 17(8), 391-400.

Lundahl, B., Moleni, T., Burke, B. L., Butters, R., Tollefson, D., Butler, C., Rollnick, S. (2013). Motivational interviewing in medical care settings: a systematic review and meta-analysis of randomized controlled trials. Patient education and counseling, 93(2), 157-168.

Lundy, L., McEvoy, L., \& Byrne, B. (2011). Working with young children as co-researchers: An approach informed by the United Nations Convention on the Rights of the Child. Early education \& development, 22(5), 714-736.

Luria, R., Sessa, P., Gotler, A., Jolicœur, P., \& Dell'Acqua, R. (2009). Visual Short-term Memory Capacity for Simple and Complex Objects. Journal of cognitive neuroscience, 22(3), 496512. doi:10.1162/jocn.2009.21214.

Maass, A. S. (2019). The Actorness of the EU's State-Building in Ukraine- Before and after Crimea. Geopolitics, 1-20.

Majerus, S., \& Van der Linden, M. (2013). Memory disorders in children. In Handbook of clinical neurology (Vol. 111, pp. 251-255). Elsevier.

Mak, W. W., \& Cheung, R. Y. (2010). Self-Stigma Among Concealable Minorities in Hong Kong: Conceptualization and Unified Measurement. American Journal of Orthopsychiatry, 80(2), 267-281.

Mammarella, I. C., Cornoldi, C., Pazzaglia, F., Toso, C., Grimoldi, M., \& Vio, C. (2006). Evidence for a double dissociation between spatial-simultaneous and spatial-sequential working memory in visuospatial (nonverbal) learning disabled children. Brain and cognition, 62(1), 58-67.

Mammarella, I. C., Pazzaglia, F., \& Cornoldi, C. (2008). Evidence for different components in children's visuospatial working memory. British Journal of Developmental Psychology, 26(3), 337-355.

Marmet, S., Rehm, J., Gmel, G., Frick, H., \& Gmel, G. (2014). Alcohol-attributable mortality in Switzerland in 2011-age-specific causes of death and impact of heavy versus non-heavy drinking. Swiss Medical Weekly, 144, w13947.

Mashiach-Eizenberg, M., Hasson-Ohayon, I., Yanos, P. T., Lysaker, P. H., \& Roe, D. (2013). Internalized stigma and quality of life among persons with severe mental illness: the mediating roles of self-esteem and hope. Psychiatry research, 208(1), 15-20. 
Masten, A. S. (2011). Resilience in children threatened by extreme adversity: Frameworks for research, practice, and translational synergy. Development and Psychopathology, 23(2), 493-506.

Masten, A. S., Burt, K. B., Roisman, G. I., Obradović, J., Long, J. D., \& Tellegen, A. (2004). Resources and resilience in the transition to adulthood: Continuity and change. Development and psychopathology, 16(4), 1071-1094.

Mattson, S. N., Roesch, S. C., Fagerlund, A., Autti-Ramo, I., Jones, K. L., May, P. A., . . . Collaborative Initiative on Fetal Alcohol Spectrum, D. (2010). Toward a neurobehavioral profile of fetal alcohol spectrum disorders. Alcohol, Clinical and Experimental Research, 34(9), 1640-1650. doi:10.1111/j.1530-0277.2010.01250.x।

Mattson, S. N., Roesch, S. C., Glass, L., Deweese, B. N., Coles, C. D., Kable, J. A., . . Cifasd. (2013). Further development of a neurobehavioral profile of fetal alcohol spectrum disorders. Alcohol, Clinical and Experimental Research, 37(3), 517-528. doi:10.1111/j.15300277.2012.01952.x.

Melby-Lervåg, M., Redick, T. S., \& Hulme, C. (2016). Working memory training does not improve performance on measures of intelligence or other measures of "far transfer" evidence from a meta-analytic review. Perspectives on Psychological Science, 11(4), 512-534.

Mezzacappa, E. (2004). Alerting, orienting, and executive attention: Developmental properties and sociodemographic correlates in an epidemiological sample of young, urban children. Child Development, 75(5), 1373-1386.

Miller, H. V., Barnes, J., \& Beaver, K. M. (2011). Self-control and health outcomes in a nationally representative sample. American journal of health behavior, 35(1), 15-27.

Miller, W. R., \& Rollnick, S. (2002). Motivational interviewing: Preparing people for change (2nd ed.). New York: Guilford Press.

Milne, S., Sheeran, P., \& Orbell, S. (2000). Prediction and intervention in health-related behavior: A meta-analytic review of protection motivation theory. Journal of Applied Social Psychology, 30(1), 106-143.

Milner, B. (1971). Interhemisoheric differences in the localization of psychological processes in man. British Medical Bulletin, 27(3), 272-277.

Mitrushina, M., Boone, K. B., Razani, J., \& D'Elia, L. F. (2005). Handbook of normative data for neuropsychological assessment: Oxford University Press.

Mittal, D., Sullivan, G., Chekuri, L., Allee, E., \& Corrigan, P. W. (2012). Empirical studies of selfstigma reduction strategies: a critical review of the literature. Psychiatric Services, 63(10), 974-981.

Mix, K. S., \& Cheng, Y. L. (2012). The relation between space and math: Developmental and educational implications. In Advances in child development and behavior (Vol. 42, pp. 197243). JAI.

Miyake, A., Friedman, N. P., Emerson, M. J., Witzki, A. H., Howerter, A., \& Wager, T. D. (2000). The unity and diversity of executive functions and their contributions to complex "Frontal Lobe" tasks: a latent variable analysis. Cognitive Psychology, 41(1), 49-100. doi:10.1006/cogp.1999.0734

Mokrushyna, H. (2015). Decentralization Reform in Ukraine. Retrieved from http://www.counterpunch.org/2015/08/28/decentralization-reform-in-ukraine/.

Murphy, J. G., Dennhardt, A. A., Skidmore, J. R., Martens, M. P., \& McDevitt-Murphy, M. E. (2010). Computerized versus motivational interviewing alcohol interventions: Impact on discrepancy, motivation, and drinking. Psychology of Addictive Behaviors, 24(4), 628. 
Naglieri, J. A. (2003). Naglieri nonverbal ability tests. In Handbook of nonverbal assessment (pp. 175189): Springer.

Naglieri, J. A., Booth, A. L., \& Winsler, A. (2004). Comparison of Hispanic children with and without limited English proficiency on the Naglieri Nonverbal Ability Test. Psychological Assessment, 16(1), 81 .

Naglieri, J. A., \& Ford, D. Y. (2003). Addressing underrepresentation of gifted minority children using the Naglieri Nonverbal Ability Test (NNAT). Gifted Child Quarterly, 47(2), 155-160.

Naglieri, J. A., \& Ronning, M. E. (2000a). Comparison of White, African American, Hispanic, and Asian children on the Naglieri Nonverbal Ability Test. Psychological Assessment, 12(3), 328.

Naglieri, J. A., \& Ronning, M. E. (2000b). The relationship between general ability using the Naglieri Nonverbal Ability Test (NNAT) and Stanford Achievement Test (SAT) reading achievement. Journal of Psychoeducational Assessment, 18(3), 230-239.

National Center for Education Statistics (2014). Digest of education statistics. Retrieved from https://nces.ed.gov/programs/digest/d14/tables/dt14_219.70.asp. Accessed July 2019.

Neuner-Jehle, S., Schmid, M., \& Grüninger, U. (2013). The "Health CCoaching" programme: a new patient-centred and visually supported approach for health behaviour change in primary care. BMC family practice, 14(1), 100.

Noble, K. G., Houston, S. M., Brito, N. H., Bartsch, H., Kan, E., Kuperman, J. M., ... \& Schork, N. J. (2015). Family income, parental education and brain structure in children and adolescents. Nature neuroscience, 18(5), 773.

Noble, K. G., McCandliss, B. D., \& Farah, M. J. (2007). Socioeconomic gradients predict individual differences in neurocognitive abilities. Developmental Science, 10(4), 464-480. doi:10.1111/j.1467-7687.2007.00600.x

Norman, M. A., Evans, J. D., Miller, W. S., \& Heaton, R. K. (2000). Demographically corrected Norms for the California verbal learning test. Journal of Clinical and Experimental neuropsychology, 22(1), 80-94.

Novikova, O., Ostafiichuk, Y., \& Khandii, O. (2019). Social Justice And Economic Efficiency Of The Modern Labour Market. Baltic Journal of Economic Studies, 5(3), 145-151.

Onwijn, K. (2005). Oekraïne. Amsterdam: KIT Publishers.

Osborne, J. W. (2010). Improving your data transformations: Applying the Box-Cox transformation. Practical Assessment, Research \& Evaluation, 15(12), 1-9.

Owen, A. M., Downes, J. J., Sahakian, B. J., Polkey, C. E., \& Robbins, T. W. (1990). Planning and spatial working memory following frontal lobe lesions in man. Neuropsychologia, 28(10), 1021-1034. doi.org/10.1016/0028-3932(90)90137-D

Ozonoff, S., Cook, I., Coon, H., Dawson, G., Joseph, R. M., Klin, A., . . Pennington, B. F. (2004). Performance on Cambridge Neuropsychological Test Automated Battery subtests sensitive to frontal lobe function in people with autistic disorder: evidence from the Collaborative Programs of Excellence in Autism network. Journal of Autism and Developmental Disorders, 34(2), 139-150.

Paluck, E. L., \& Green, D. P. (2009). Prejudice reduction: What works? A review and assessment of research and practice. Annual review of psychology, 60, 339-367.

Palmeri, T. J., \& Tarr, M. (2008). Visual object perception and long-term memory. Visual memory, 163-207. 
Pangelinan, M. M., Zhang, G., VanMeter, J. W., Clark, J. E., Hatfield, B. D., \& Haufler, A. J. (2011). Beyond age and gender: relationships between cortical and subcortical brain volume and cognitive-motor abilities in school-age children. Neuroimage, 54(4), 3093-3100.

Parsons, S., \& Bynner, J. (1997). Numeracy and employment. Education+ Training, 39(2), 43-51.

Paule, M. G., Bushnell, P. J., Maurissen, J. P., Wenger, G. R., Buccafusco, J. J., Chelonis, J. J., \& Elliott, R. (1998). Symposium overview: the use of delayed matching-to-sample procedures in studies of short-term memory in animals and humans. Neurotoxicology and teratology, 20(5), 493-502.

Pedersen, E. R., Miles, J. N., Ewing, B. A., Shih, R. A., Tucker, J. S., \& D’Amico, E. J. (2013). A longitudinal examination of alcohol, marijuana, and cigarette perceived norms among middle school adolescents. Drug and alcohol dependence, 133(2), 647-653.

Peeters, M., Janssen, T., Monshouwer, K., Boendermaker, W., Pronk, T., Wiers, R., \& Vollebergh, W. (2015). Weaknesses in executive functioning predict the initiating of adolescents' alcohol use. Developmental cognitive neuroscience, 16, 139-146.

Perlman, S. B., Huppert, T. J., \& Luna, B. (2015). Functional near-infrared spectroscopy evidence for development of prefrontal engagement in working memory in early through middle childhood. Cerebral Cortex, bhv139.

Pescosolido, B. A., \& Boyer, C. A. (2010). Understanding the context and dynamic social processes of mental health treatment. A handbook for the study of mental health: Social contexts, theories, and systems, 2, 420-438.

Pescosolido, B. A., \& Martin, J. K. (2015). The stigma complex. Annual review of sociology, 41, 87116.

Pescosolido, B. A., Martin, J. K., Long, J. S., Medina, T. R., Phelan, J. C., \& Link, B. G. (2010). “A disease like any other"? A decade of change in public reactions to schizophrenia, depression, and alcohol dependence. American Journal of Psychiatry, 167(11), 1321-1330.

Phelan, J. C., Link, B. G., \& Dovidio, J. F. (2008). Stigma and prejudice: one animal or two? Social Science \& Medicine, 67(3), 358-367.

Phelan, J. C., Link, B. G., \& Tehranifar, P. (2010). Social conditions as fundamental causes of health inequalities: theory, evidence, and policy implications. Journal of health and social behavior, 51(1_suppl), S28-S40.

Piechatzek, M., Indlekofer, F., Daamen, M., Glasmacher, C., Lieb, R., Pfister, H., . . Schütz, C. G. (2009). Is moderate substance use associated with altered executive functioning in a population-based sample of young adults? Human Psychopharmacology: Clinical and Experimental, 24(8), 650-665. doi:10.1002/hup.1069.

Polshkova, S., Chaban, O., \& Walton, M. A. (2016). Alcohol use, depression, and high-risk occupations among young adults in the Ukraine. Substance Use \& Misuse, 51(7), 948-951.

Popova, S., Lange, S., Shield, K., Burd, L., \& Rehm, J. (2019). Prevalence of fetal alcohol spectrum disorder among special subpopulations: a systematic review and meta-analysis. Addiction 114(7), 1150-1172.

Popova, S., Rehm, J., Patra, J., \& Zatonski, W. (2007). Comparing alcohol consumption in central and eastern Europe to other European countries. Alcohol \& Alcoholism, 42(5), 465-473. doi:10.1093/alcalc/agl124.

Quinn, J. G. (1994). Towards a clarification of spatial processing. The Quarterly Journal of experimental Psychology, 47(2), 465-480. 
Raghubar, K. P., Barnes, M. A., \& Hecht, S. A. (2010). Working memory and mathematics: A review of developmental, individual difference, and cognitive approaches. Learning and individual differences, 20(2), 110-122.

Raven, J. (2000). The Raven's progressive matrices: change and stability over culture and time. Cognitive psychology, 41(1), 1-48.

Rasmussen, C., Soleimani, M., \& Pei, J. (2011). Executive functioning and working memory deficits on the $\mathrm{CANTAB} \circledast$ among children with prenatal alcohol exposure. Journal of population therapeutics and clinical pharmacology, 18(1).

Rauscher, F. H., Shaw, G., Levine, L., Wright, E., Dennis, W., \& Newcomb, R. (1997). Music training causes long-term enhancement of preschool children's spatial-temporal reasoning. Neurological research, 19(1), 2-8.

Rehm, J., Baliunas, D., Borges, G. L., Graham, K., Irving, H., Kehoe, T., . . Taylor, B. (2010). The relation between different dimensions of alcohol consumption and burden of disease: an overview. Addiction, 105(5), 817-843. doi:10.1111/j.1360-0443.2010.02899.x

Rehm, J., Mathers, C., Popova, S., Thavorncharoensap, M., Teerawattananon, Y., \& Patra, J. (2009). Global burden of disease and injury and economic cost attributable to alcohol use and alcohol-use disorders. Lancet, 373(9682), 2223-2233. doi:10.1016/s0140-6736(09)60746-7

Rehm, J., Shield, K. D., Gmel, G., Rehm, M. X., \& Frick, U. (2013). Modeling the impact of alcohol dependence on mortality burden and the effect of available treatment interventions in the European Union. European Neuropsychopharmacology, 23(2), 89-97. D doi.org/10.1016/j.euroneuro.2012.08.001.

Reitan, T. C. (2001). The operation failed, but the patient survived. Varying assessments of the Soviet Union's last anti-alcohol campaign1. Communist and post-communist studies, 34(2), 241260.

Resch, C., Rosema, S., Hurks, P., de Kloet, A., \& van Heugten, C. (2018). Searching for effective components of cognitive rehabilitation for children and adolescents with acquired brain injury: A systematic review. Brain injury, 32(6), 679-692.

Resing, W. C. M., \& Bleichroth, N. (2015). RAKIT-2 Intelligentiemeting bij kinderen in de basisschool leeftijd. In Resing, W.C.M. (Ed), Handboek intelligentietheorie en testgebruik (141-168). Amsterdam: Pearson.

Richardson, J. T. E. (2005). Knox's cube imitation test: A historical review and an experimental analysis. Brain and Cognition, 59(2), 183-213. doi.org/10.1016/j.bandc.2005.06.001

Robbins, T. W., James, M., Owen, A. M., Sahakian, B. J., Lawrence, A. D., McInnes, L., \& Rabbitt, P. M. (1998). A study of performance on tests from the CANTAB battery sensitive to frontal lobe dysfunction in a large sample of normal volunteers: Implications for theories of executive functioning and cognitive aging. Journal of the International Neuropsychological Society, 4(05), 474-490.

Rojahn, J., \& Naglieri, J. A. (2006). Developmental gender differences on the Naglieri Nonverbal Ability Test in a nationally normed sample of 5-17 year olds. Intelligence, 34(3), 253-260.

Romano, M., \& Peters, L. (2015). Evaluating the mechanisms of change in motivational interviewing in the treatment of mental health problems: A review and meta-analysis. Clinical Psychology Review, 38, 1-12.

Room, R. (2005). Stigma, social inequality and alcohol and drug use. Drug Alcohol Review, 24. 
Roozen, S., Peters, G. J. Y., Kok, G., Townend, D., Nijhuis, J., \& Curfs, L. (2016). Worldwide prevalence of fetal alcohol spectrum disorders: A systematic literature review including meta-analysis. Alcoholism: Clinical and Experimental Research, 40(1), 18-32.

Roque, D. T., Teixeira, R. A. A., Zachi, E. C., \& Ventura, D. F. (2011). The use of the Cambridge Neuropsychological Test Automated Battery (CANTAB) in neuropsychological assessment: Application in Brazilian research with control children and adults with neurological disorders. Psychology \& Neuroscience, 4(2), 255.

Rosselli, M., \& Ardila, A. (2003). The impact of culture and education on non-verbal neuropsychological measurements: A critical review. Brain and Cognition, 52(3), 326-333. doi.org/10.1016/S0278-2626(03)00170-2.

Rueda, M. R., Posner, M. I., \& Rothbart, M. K. (2005). The development of executive attention: Contributions to the emergence of self-regulation. Developmental neuropsychology, 28(2), 573-594.

Ruiter, R. A., Kessels, L. T., Peters, G. J. Y., \& Kok, G. (2014). Sixty years of fear appeal research: Current state of the evidence. International journal of psychology, 49(2), 63-70.

Sachse, M., Schlitt, S., Hainz, D., Ciaramidaro, A., Schirman, S., Walter, H., . . Freitag, C. M. (2013). Executive and visuo-motor function in adolescents and adults with autism spectrum disorder. Journal of Autism Developmental Disorders, 43(5), 1222-1235. doi:10.1007/s10803-012-1668-8.

Sahakian, B. J., \& Owen, A. M. (1992). Computerized assessment in neuropsychiatry using CANTAB: discussion paper. Journal of the Royal Society of Medicine, 85(7), 399.

Salmanian, M., Tehrani-Doost, M., Ghanbari-Motlagh, M., \& Shahrivar, Z. (2012). Visual memory of meaningless shapes in children and adolescents with autism spectrum disorders. Iranian journal of psychiatry, 7(3), 104.

Schittekatte, M. \& Hurks, P. (2015). WISC-III Intelligentiemeting bij kinderen en adolescenten. In R Resing, W.C.M. (Ed), Handboek intelligentietheorie en testgebruik (169-198). Amsterdam: Pearson.

Schomerus, G., Corrigan, P. W., Klauer, T., Kuwert, P., Freyberger, H. J., \& Lucht, M. (2011). Selfstigma in alcohol dependence: consequences for drinking-refusal self-efficacy. Drug \& Alcohol Dependence, 114(1), 12-17.

Schomerus, G., Lucht, M., Holzinger, A., Matschinger, H., Carta, M. G., \& Angermeyer, M. C. (2010). The stigma of alcohol dependence compared with other mental disorders: a review of population studies. Alcohol Alcohol, 46(2), 105-112.

Shallice, T. (1982). Specific Impairments of Planning. Philosophical Transactions of the Royal Society of London B: Biological Sciences, 298(1089), 199-209.

Shaw, P., Greenstein, D., Lerch, J., Clasen, L., Lenroot, R., Gogtay, N., . . Giedd, J. (2006). I Intellectual ability and cortical development in children and adolescents. Nature, 440(7084), 676-679.

Sinha, R. (2009). Stress and Addiction: A Dynamic Interplay of Genes, Environment, and Drug Intake. Biological Psychiatry, 66(2), 100-101. doi.org/10.1016/j.biopsych.2009.05.003.

Sigvardsson, S., Bohman, M., \& Cloninger, C. R. (1996). Replication of the Stockholm Adoption Study of alcoholism: Confirmatory cross-fostering analysis. Archives of general psychiatry, 53(8), 681-687.

Sluzenski, J., Newcombe, N. S., \& Satlow, E. (2004). Knowing where things are in the second year of life: Implications for hippocampal development. Journal of Cognitive Neuroscience, 16(8), 1443-1451. 
Smith, E. E., Jonides, J., \& Koeppe, R. A. (1996). Dissociating verbal and spatial working memory using PET. Cerebral cortex, 6(1), 11-20.

Smith, P. J., Need, A. C., Cirulli, E. T., Chiba-Falek, O., \& Attix, D. K. (2013). A comparison of the Cambridge Automated Neuropsychological Test Battery (CANTAB) with traditional" neuropsychological testing instruments. Journal of clinical and experimental neuropsychology, 35(3), 319-328.

Steinhausen, H.-C., \& Spohr, H.-L. (1998). Long-Term Outcome of Children with Fetal Alcohol Syndrome: Psychopathology, Behavior, and Intelligence. Alcoholism: Clinical and E Experimental Research, 22(2), 334-338. doi:10.1111/j.1530-0277.1998.tb03657.x.

Strauss, E., Sherman, E. M., \& Spreen, O. (2006). A compendium of neuropsychological tests: Administration, norms, and commentary: Oxford University Press, USA.

Stutterheim, S. E., Baas, I., Roberts, H., Brands, R., Schmidt, J., Lechner, L., ... \& Bos, A. E. (2016) Stigma experiences among substance users with HIV. Stigma and Health, 1 (3), 123.

Stutterheim, S. E., Pryor, J. B., Bos, A. E., Hoogendijk, R., Muris, P., \& Schaalma, H. P. (2009). HIVrelated stigma and psychological distress: the harmful effects of specific stigma manifestations in various social settings. Aids, 23(17), 2353-2357.

Subtelny, O. (2009). Ukraine: a history. Toronto: University of Toronto Press.

Tallberg, I. M., Ivachova, E., Jones Tinghag, K., \& Östberg, P. (2008). Swedish norms for word fluency tests: FAS, animals and verbs.Scandinavian journal of psychology, 49(5), 479-485.

Tau, G. Z., \& Peterson, B. S. (2010). Normal development of brain circuits. Neuropsychopharmacology, 35(1), 147.

Taylor, M. J., \& Heaton, R. K. (2001). Sensitivity and specificity of WAIS-III/WMS-III demographically corrected factor scores in neuropsychological assessment. Journal of the International Neuropsychological Society, 7(7), 867-874.

Teixeira, R. A. A., Zachi, E. C., Roque, D. T., Taub, A., \& Ventura, D. F. (2011). Memory span measured by the spatial span tests of the Cambridge Neuropsychological Test Automated Battery in a group of Brazilian children and adolescents. Dementia \& Neuropsychologia, 5(2), 129-134.

The World Bank (2016). Rural population (\% of total population) by country. Retrieved from https://data.worldbank.org/indicator/SP.RUR.TOTL.ZS?locations=UA. Retrieved February 2, 2017.

Theron, L. C., Theron, A. M., \& Malindi, M. J. (2013). Toward an African definition of resilience: A rural South African community's view of resilient Basotho youth. Journal of Black Psychology, 39(1), 63-87.

Thomas, D. R. (2003). A general inductive approach for qualitative data analysis: School of Population Health. University of Auckland, New Zealand.

Thomas, M. S., Annaz, D., Ansari, D., Scerif, G., Jarrold, C., \& Karmiloff-Smith, A. (2009). Using developmental trajectories to understand developmental disorders. Journal of speech, language, and hearing research, 52(2), 336-358.

Thomas, M., \& Johnson, M. (2008). New advances in understanding sensitive periods in brain development. Current Directions in Psychological Science, 17, 1-5.

Thornicroft, G., Mehta, N., Clement, S., Evans-Lacko, S., Doherty, M., Rose, D., ... \& Henderson, C. (2016). Evidence for effective interventions to reduce mental-health-related stigma and discrimination. The Lancet, 387(10023), 1123-1132. 
Toornstra, A., Hurks, P. P., Van der Elst, W., Kok, G., \& Curfs, L. M. (2019). Measuring visual matching and short-term recognition memory with the CANTAB® Delayed Matching to Sample task in schoolchildren: Effects of demographic influences, multiple outcome measures and regression-based normative data. Child Neuropsychology, 1-30. doi.org/10.1080/09297049.2019.1642316

Toornstra, A., Massar, K., Hurks, P. P. M.,Timmermans, M. M. M. S., Kok, G., \& Curfs, L.M.G. (resubmitted). Perceptions of alcohol use in Ukraine: A qualitative investigation among community members and youths.

Ukraine Channel (2017). Retrieved from http://www.ukraine.com/education/

United Nations Educational, Scientific and Cultural Organization, UNESCO (1994). The Salamanca Statement and Framework for Action on Special Needs Education adopted by the Special Needs Conference on Special Needs Education: Access and Quality. Retrieved from http://unesdoc.unesco.org/images/0009/000984/098427eo.pdf.

Vaillancourt, T., Duku, E., Becker, S., Schmidt, L. A., Nicol, J., Muir, C., \& MacMillan, H. (2011) Peer victimization, depressive symptoms, and high salivary cortisol predict poorer memory in children. Brain and Cognition, 77(2), 191-199.

Van Breukelen, G. J. P., \& Vlaeyen, J. W. S. (2005). Norming clinical questionnaires with multiple regression: The Pain Cognition List. Psychological Assessment, 17(3), 336-344. doi:10.1037/1040-3590.17.3.336

Van der Elst, W., Hurks, P., Wassenberg, R., Meijs, C., \& Jolles, J. (2011). Animal Verbal Fluency and Design Fluency in school-aged children: Effects of age, sex, and mean level of parental education, and regression-based normative data. Journal of Clinical and Experimental Neuropsychology, 33(9), 1005-1015. doi:10.1080/13803395.2011.589509

Van der Elst, W., Molenberghs, G., Hilgers, R. D., Verbeke, G., \& Heussen, N. (2016). Estimating the reliability of repeatedly measured endpoints based on linear mixed-effects models. A tutorial. Pharmaceutical statistics, 15(6), 486-493.

Van der Elst, W., Ouwehand, C., van der Werf, G., Kuyper, H., Lee, N., \& Jolles, J. (2012). The Amsterdam Executive Function Inventory (AEFI): psychometric properties and demographically corrected normative data for adolescents aged between 15 and 18 years. Journal of clinical and experimental neuropsychology, 34(2), 160-171. doi.org/10. $10911017 /$ S1355617705050344

Van der Elst, W., van Boxtel, M. P., van Breukelen, G. J., \& Jolles, J. (2005). Rey's verbal learning test: normative data for 1855 healthy participants aged 24-81 years and the influence of age, sex, education, and mode of presentation. Journal of the International Neuropsychological Society, 11(3), 290-302. doi:10.1017/S1355617705050344

Vinţan, M. A., Palade, S., Cristea, A., Benga, I., \& Muresanu, D. F. (2012). A neuropsychological assessment, using computerized battery tests (CANTAB), in children with benign rolandic epilepsy before AED therapy. Journal of medicine and life, 5(1), 114.

Visu-Petra, L., Benga, O., Tincaş, I., \& Miclea, M. (2007). Visual-spatial processing in children and adolescents with Down's syndrome: a computerized assessment of memory skills. Journal of Intellectual Disability Research, 51(12), 942-952.

Voyer, D., Voyer, S. D., \& Saint-Aubin, J. (2017). Sex differences in visual-spatial working memory: A meta-analysis. Psychonomic Bulletin \& Review, 24(2), 307-334. doi:10.3758/s13423-016$1085-7$

Ware, A. L., Infante, M. A., O'Brien, J. W., Tapert, S. F., Jones, K. L., Riley, E. P., \& Mattson, S. N. (2015). An fMRI study of behavioral response inhibition in adolescents with and without 
histories of heavy prenatal alcohol exposure. Behavioural Brain Research, 278, 137-146. doi:10.1016/j.bbr.2014.09.037

Wassenberg, R., Hendriksen, J. G., Hurks, P. P., Feron, F. J., Vles, J. S., \& Jolles, J. (2008). Speed of language comprehension is impaired in ADHD. Journal of Attention Disorders.

Wasserman, D., Värnik, A., \& Eklund, G. (1994). Male suicides and alcohol consumption in the former USSR. Acta Psychiatrica Scandinavica, 89(5), 306-313.

Wasserman, D., Värnik, A., \& Eklund, G. (1998). Female suicides and alcohol consumption during perestroika in the former USSR. Acta Psychiatrica Scandinavica, 98, 26-33.

Weare, K., \& Nind, M. (2011). Mental health promotion and problem prevention in schools: what does the evidence say? Health promotion international, 26(suppl_1), i29-i69.

Webb, C. P., Bromet, E. J., Gluzman, S., Tintle, N. L., Schwartz, J. E., Kostyuchenko, S., \& Havenaar, J. M. (2005). Epidemiology of heavy alcohol use in Ukraine: findings from the world mental health survey. Alcohol and Alcoholism, 40(4), 327-335.

Wechsler, H., \& Kuo, M. (2000). College students define binge drinking and estimate its prevalence: Results of a national survey. Journal of American College Health, 49(2), 57-64.

Weiss, M. G., Ramakrishna, J., \& Somma, D. (2006). Health-related stigma: rethinking concepts and interventions. Psychology, health \& medicine, 11(3), 277-287.

Wells, J., Barlow, J., \& Stewart-Brown, S. (2003). A systematic review of universal approaches to mental health promotion in schools. Health Education, 103(4), 197-220.

White, K. R. (1982). The relation between socioeconomic status and academic achievement. Psychological Bulletin, 91(3), 461.

Windle, M. (2000). Parental, sibling, and peer influences on adolescent substance use and alcohol problems. Applied Developmental Science, 4(2), 98-110.

Wolfe, J. M., Kluender, K. R., Levi, D. M., Bartoshuk, L. M., Herz, R. S., Klatzky, R., . . Merfled, D. M. (2015). Sensation and Perception (4 ed.). Massachusetts U.S.A.: Sinauer Associates, Inc.

Woods, D. L., Wyma, J. M., Yund, E. W., Herron, T. J., \& Reed, B. (2015). Factors influencing the latency of simple reaction time. Frontiers in Human Neuroscience, 9, 131. doi:10.3389/fnhum.2015.00131.

World Health Organisation (WHO; 2014). Global status report on alcohol and health. Retrieved from http://www.who.int/substance_abuse/publications/global_alcohol_report/en/ Accessed March 1, 2016.

World Health Organisation (WHO; 2018). Global status report on alcohol and health. Retrieved from https://apps.who.int/iris/bitstream/handle/10665/274603/9789241565639-eng.pdf. Accessed September 21, 2019.

World Medical Association Declaration of Helsinki (2013). Ethical principles for medical research involving human subjects. JAMA, 310, 2191-2194.

Zachary, R. A., \& Gorsuch R. L. (1985). Continuous norming: Implications for the WAIS-R. Journal of Clinical Psychology, 41, 86-94.

Zelazo, P. D., Blair, C. B., \& Willoughby, M. T. (2016). Executive Function: Implications for Education. NCER 2017-2000. National Center for Education Research. 
Zasenko, O.E., Stebelsky, I., Hajda, L.A., Kryzhaniysky, S.A., Makuch, A., \& Yerofeyev, I.A. (2017). Ukraine. Encyclopaedia Britanica.Retrieved from https://www.britannica.com/place/Ukraine.

Zhou, M. (1998). Empirical Distributions. Retrieved from http://www.ms.uky.edu/\%7Emai/java/stat/EmpDis.html.

Zimmerman, B. J. (2001). Theories of self-regulated learning and academic achievement: An overview nd analysis (p. 1-36). In Zimmerman, B. J., and Schunk, D. (Eds.). Self-Regulated Learning nd Academic Achievement. New York: Routledge.

Zucker, R. A., Donovan, J. E., Masten, A. S., Mattson, M. E., \& Moss, H. B. (2008). Early developmental processes and the continuity of risk for underage drinking and problem drinking. Pediatrics, 121 Supplement 4, S252-272. doi:10.1542/peds.2007-2243B. 


\section{Acknowledgements}

\section{Gratitude}

Coming to the last pages of this dissertation is a special feeling. I would like to thank all at Maastricht University who made this possible. It has changed my life. I wonder how you find the courage to train a brain like mine with so much experience yet little of the rigour and structure that results in the quality needed for academic work. I am grateful. The department of Social, Work and Organizational Psychology has supported my endavour more than I could have hoped for. A special word for my co-supervisors Petra Hurks and Karlijn Massar whose endless patience and art of guiding through asking questions and nudging toward structure has and will be a tremendous source of inspiration. Statistics support from Wim van der Elst has been invaluable, especially the insights in dealing with the characteristics of data collected in the field.

I have repeatedly been given the chance in education, the world over, to adapt learning programmes for (individual) academic success. Creating the building blocks one at a time, preferably for a daily successful experience. Yet, health and advantageous life outcomes appeared sometimes so much out of reach, especially when other educational or environmental pressures arose. Learning the Intervention Mapping perspective and approaches, theory and evidence - my gratitude to Gerjo Kok and Nell Gottlieb cannot be captured in words. Also, thanks to Leopold Curfs who kept the overview and noticed disadvantageous avenues in specialist topics. My appreciation extends to Sarah Stutterheim who gave her time and knowledge to proofread chapter 6.

A special thanks to the foundations Halm and SHOK. The latter financed the translations, while the first contributed to the small teacher training pilot of Kindergarten teachers. Most of all, the host Kindergarten who so generously gave time to share with colleagues and discuss views and challenges in teaching early years. David and the community: your organization has surpassed all expectations. The pilot also included coteaching and Kindergarten materials allowing us to explore 'Piagetan' materials, which again I may tell in gratefulness, were donated later. A special thanks to James from Cambridge for fair and open negotiations and providing me the tests within my budget. The list is long and I would like to thank all who I might have forgotten to mention by name and/or who hopefully know my profound gratitude. To mention a few; Phia, Elles, Annie, Reina, Dorien and her colleague, Jozo, Szabina, Eric, Stacey, Margreet and family. Maxime and Eva; Christmas office decorations have never quite been the same! Driving to Ukraine and through Ukraine was a privilege and possible with as some started to call it in local language 'the little carriage'. Thank you to family and friends who helped me make that possible.

Thank you to all children and youth; many wonderful encounters. I can only try to find words for what participants shared. One respondent wanted to tell the story, taking risk for upsetting the delicate atmosphere in their house. Supported by community members, they wanted to use their agency to tell their story. I hope I conveyed the story you wanted the world to know. 


\section{Curriculum Vitae}

Personal data:

Annet Toornstra

annet.toornstra@maastrichtuniversity.nl 0622152314

\section{Education}

2018 BKO University Teaching Qualification

$2018 \quad$ Motivational Interviewing basic qualification

2015 Psychometric Testing Qualification (British Psychological Society)

2012 Master of Educational Neuroscience Birkbeck University, London

2009 Master of Education (specialisation self-regulation) including 4

Master of Business Administration modules. Griffith University,

Brisbane

Teaching Experience

2016 - current Teacher FPN Maastrict University (including coordinator Cognitive-

Psychology; course planning group Personality and Individual

Differences; Mentalpreneurship)

2013 - $2015 \quad$ Substitute \& special needs teacher 6 - 17 years old

2011 - 2013 Substitute teacher Greater London (e.g., Evelina's children hospital)

2008-2011 Head academic learning support Vocational College for students with learning difficulties, Johannesburg, South Africa. Responsible for all student exam administration in NVQ, placement testing \& exam preparation, school assessment \& portfolio supervision. Educational psychological testing of all students (supervised by the University of Johannesburg) for subsidised work placement.

Research Experience

$2012 \quad$ Numbersense and positive affect in adolescents with learning difficulties in South Africa (unpublished master thesis)

2009 Self-regulation in literacy; a qualitative case study of a student with learning difficulties (unpublished master thesis)

\section{Publications}

Toornstra, A., Hurks, P. P. M., Van der Elst, W., Kok, G., \& Curfs, L. M. G. (2019). Measuring visual matching and short-term recognition memory with the CANTAB ${ }^{\circledR}$ Delayed Matching to Sample task in schoolchildren: Effects of demographic influences, multiple outcome measures and regression based normative data. Child Neuropsychology, 1-30. doi.org/10.1080/09297049.2019.1642316 
Toornstra, A., Hurks, P. P. M., Van der Elst, W., Kok, G., \& Curfs, L. M. G. (2019).

Measuring Visual, Spatial, and Visual Spatial Short-Term Memory in Schoolchildren:

Studying the Influence of Demographic Factors and Regression-Based Normative Data.

Journal of Pediatric Neuropsychology. doi 10.1007/s40817-019-00070-6

\section{Resubmitted}

Toornstra, A., Massar, K., Hurks, P. P. M.,Timmermans, M. M. M. S., Kok, G., \& Curfs, L.M.G. Perceptions of alcohol use in Ukraine: A qualitative investigation among community members and youths. 


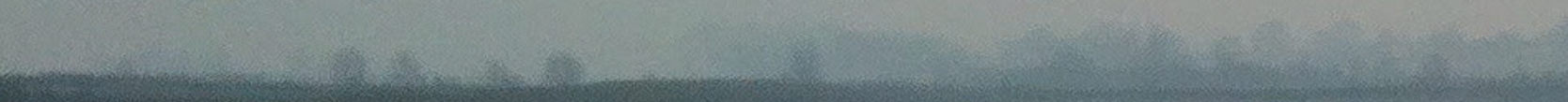

